Prepared for the U.S. Department of Energy

under Contract DE-AC05-76RL01830

\title{
Benchmark Problems of the Geothermal Technologies Office Code Comparison Study
}
M.D. White
M.W. McClure
P. Fu
Q. Cheng
D. Elsworth
Q. Gan
Y. Hao
K.J. Im
R. Safari
Q. Tao
Y. Xia

November 2016
R.K. Podgorney
G. Danko
D. Bahrami
K. Chiu
Y. Fang
Q. Gao
R.N. Horne
J. Norbeck
V. Sesetty
S.K. White

S. Kelkar

A. Ghassemi

C. Barbier

C. Detournay

J.K. Furtney

B. Guo

K. Huang

J. Rutqvist

E. Sonnenthal

Z. Wong 


\title{
DISCLAIMER
}

This report was prepared as an account of work sponsored by an agency of the United States Government. Neither the United States Government nor any agency thereof, nor Battelle Memorial Institute, nor any of their employees, makes any warranty, express or implied, or assumes any legal liability or responsibility for the accuracy, completeness, or usefulness of any information, apparatus, product, or process disclosed, or represents that its use would not infringe privately owned rights. Reference herein to any specific commercial product, process, or service by trade name, trademark, manufacturer, or otherwise does not necessarily constitute or imply its endorsement, recommendation, or favoring by the United States Government or any agency thereof, or Battelle Memorial Institute. The views and opinions of authors expressed herein do not necessarily state or reflect those of the United States Government or any agency thereof.

\author{
PACIFIC NORTHWEST NATIONAL LABORATORY \\ operated by \\ BATTELLE \\ for the \\ UNITED STATES DEPARTMENT OF ENERGY \\ under Contract DE-AC05-76RL01830
}

Printed in the United States of America

Available to DOE and DOE contractors from the

Office of Scientific and Technical Information,

P.O. Box 62, Oak Ridge, TN 37831-0062;

ph: (865) 576-8401

fax: $(865)$ 576-5728

email: reports@adonis.osti.gov

Available to the public from the National Technical Information Service

5301 Shawnee Rd., Alexandria, VA 22312

ph: (800) 553-NTIS (6847)

email: ordersantis.gov <http://www.ntis.gov/about/form.aspx>

Online ordering: http://www.ntis.gov

This document was printed on recycled paper.

(8/2010) 


\section{Benchmark Problems of the Geothermal Technologies Office Code Comparison Study}

$\begin{array}{lll}\text { M.D. White } & \text { R.K. Podgorney } & \text { S. Kelkar } \\ \text { M.W. McClure } & \text { G. Danko } & \text { A. Ghassemi } \\ \text { P. Fu } & \text { D. Bahrami } & \text { C. Barbier } \\ \text { C. Detournay } & \text { D. Elsworth } & \text { B. Guo } \\ \text { R. Horne } & \text { J.K. Furtney } & \text { J. Norbeck } \\ \text { J. Rutqvist } & \text { E. Sonnenthal } & \text { S.K. White } \\ \text { Q. Cheng } & \text { K. Chiu } & \text { Y. Fang } \\ \text { Q. Gan } & \text { Q. Gao } & \text { Y. Hao } \\ \text { K. Huang } & \text { K.J. Im } & \text { R. Safari } \\ \text { V. Sesetty } & \text { Q. Tao } & \text { Z. Wong } \\ \text { Y. Xia } & & \end{array}$

November 2016

Prepared for

the U.S. Department of Energy

under Contract DE-AC05-76RL01830

Pacific Northwest National Laboratory

Richland, Washington 99352 



\section{Abstract}

A diverse suite of numerical simulators is currently being applied to predict or understand the performance of enhanced geothermal systems (EGS). To build confidence and identify critical development needs for these analytical tools, the United States Department of Energy, Geothermal Technologies Office has sponsored a Code Comparison Study (GTO-CCS), with participating teams representing universities, industry, and U.S. national laboratories. A principal objective for the study was to create a community forum for improvement and verification of numerical simulators for EGS modeling. Each participating team brought unique numerical simulation capabilities to bear on the problems. To accurately characterize the state of knowledge and simulation capabilities, two classes of problems were developed during the study, including benchmark problems and challenge problems. The benchmark problems were structured to test the ability of the collection of numerical simulators to solve various combinations of coupled thermal, hydrologic, geomechanical, and geochemical processes. This class of problems was strictly defined in terms of properties, driving forces, initial conditions, and boundary conditions. Study participants submitted solutions to problems for which their simulation tools were deemed capable or nearly capable. Some participating codes were originally developed for EGS applications whereas some others were designed for different applications but can simulate processes similar to those in EGS. Submissions of solution from both types of codes were encouraged. In some cases, participants made small incremental changes to their numerical simulation codes to address specific elements of the problem, and in other cases participants submitted solutions with existing simulation tools, acknowledging the limitations of the code. The challenge problems were based on the enhanced geothermal systems research conducted at Fenton Hill, near Los Alamos, New Mexico, between 1974 and 1995. The problems involved two phases of research, stimulation, development, and circulation in two separate reservoirs. The challenge problems had specific questions to be answered via numerical simulation in three topical areas: 1) reservoir creation/stimulation, 2) reactive and passive transport, and 3 ) thermal recovery. Whereas the benchmark class of problems were designed to test capabilities for modeling coupled processes under strictly specified conditions, the stated objective for the challenge class of problems was to demonstrate what new understanding of the Fenton Hill experiments could be realized via the application of modern numerical simulation tools by recognized expert practitioners.

This report begins with a general description of the mathematical formulations and numerical solution schemes of modern numerical simulators for modeling EGS reservoirs. Next detailed descriptions of the individual numerical simulators applied during the GTO-CCS are presented highlighting coupled process modeling capabilities, governing mathematical models, numerical solution techniques, multiple processor computing approaches, and development history. A selective list of applications outside of the GTO-CCS are additionally presented for each numerical simulator used during the study: AD-GPRS (AutomaticDifferentiation General Purpose Research Simulator), CFRAC (Complex Fracturing ReseArch Code), FALCON (Fracturing And Liquid CONvection), FEHM (Finite Element Heat and Mass), FLAC ${ }^{3 \mathrm{D}}$ (Fast Lagrangian Analysis of Continua in 3-Dimensions), GeoFrac-Mech, GeoFrac-Stim, GEOS (open source massively parallel code), MULTIFLUX, NUFT (Nonisothermal Unsaturated-saturated Flow and Transport), STOMP (Subsurface Transport Over Multiple Phases), TOUGH2, TOUGH-FLAC (TOUGH2 and FLAC3D sequentially linked), TOUGHREACT (TOUGH2 with REACtive Transport), TOUGHREACT-ROCMECH (TOUGHREACT and ROCMECH sequentially linked), TR_FLAC3D (TOUGHREACT and FLAC3D sequentially linked), UDEC (Universal Distinct Element Code). 
These introductory sections are then followed with descriptions, solutions, and comparisons for the seven benchmark problems. The comparison standard, ISO-13258, developed by the chemical and physical measurement communities for proficiency testing was applied to quantify the uncertainty in simulation results among the participants and codes. Problem 1 involved a poro-elastic fault layer in lieu of a single, robust fracture in a geothermal reservoir under water injection, resembling the experimental site at the Raft River EGS demonstration in southern Idaho. The observed reservoir behavior includes a strong non-linear response between the injection rates and pressures over multiple-day, variable-rate injection tests. The behavior is simplified with the assumption of the permeability being an exponential function of hydrodynamic pressure in the inferred fault zone. Problem 2 involved the injection of cold water at a specified pressure at one location inside a three-dimensional domain with outside boundaries held fixed at initial conditions of pressure, temperature and far-field stresses. This problem was motivated by the shear stimulation treatment of the well Desert Peak 27-15 conducted in September 2010 and the subsequent modeling analysis. Problem 3 involved injection into three fractures and investigated the coupling of fluid flow, fracture shear, and fracture opening. Problem 4 considered the responses of a single planar fracture in the rock based on the first experimental EGS in the U.S. at Fenton Hill, Phase I. The thermal-hydrologic-mechanical (THM) responses of this fracture during a 24-day injection and production period were simulated. The rock mechanics model component includes the thermo-elastic response of the self-propped fracture layer coupled to the thermal model of the reservoir during coolant injection. Problem 5 considered the flow of water through an idealized horizontal fracture with temperature dependent reaction of the water with amorphous silica, the principal mineral constituent of the formation. The dissolution and precipitation of amorphous silica along the length of the fracture zone is to be computed for two forms of injected water: 1) pure water and 2) recycled water. Changes in intrinsic permeability and porosity due to mineral dissolution and precipitation are also considered. A relationship between changes in porosity and intrinsic permeability was used that considered the effects of pore-throat clogging by precipitates. Problem 6 involved injection into a fault surrounded by a porothermoelastic medium. Problem 7 entails the calculation of ground surface deformation caused by a pressurized subsurface fracture. Surface deformation, usually measured using interferometric synthetic aperture radar (InSAR) or tiltmeter, provides an important means to infer reservoir condition, particularly fracture extent development. A rectangular shaped fracture with various dipping angles $\left(0^{\circ}, 45^{\circ}\right.$, and $\left.90^{\circ}\right)$ is considered, and both 2D and 3D solutions are compared. 


\section{Summary}

Scientists and engineers have sought to develop numerical models of geologic systems for their inherent ability to yield quantitative predictions, but more importantly to understand complex coupled processes. Enhanced geothermal systems (EGSs) require the development of hydraulic connection across wells drilled into hot dry impermeable rock, via the opening of natural fractures, the creation of new hydraulic fractures, or a mixture of both mechanisms. Numerical simulation of this stimulation stage of an EGS requires modeling capabilities for tightly coupled thermal, hydrologic, and geomechanical (THM) processes. Once created the recovery of thermal energy from an EGS system involves the circulation of a working fluid through the reservoir, with injection and production pressures being critical controlling parameters. EGS reservoirs are dynamic systems, with changes occurring in response to controlling pressures, decreasing temperature over time, and alterations in the hydrologic network via dissolution and precipitation of the formation minerals in response to fresh-water injection and temperature transitions across the reservoir. Numerical simulation of the thermal recovery stage of an EGS system, therefore, requires modeling capabilities for tightly coupled thermal, hydrologic, geomechanical, and geochemical (THMC) processes. Numerical simulators have evolved over the last three decades, since the first formal comparison of codes for geothermal systems at the Sixth Workshop on Geothermal Reservoir Engineering held on the Stanford University in 1980, to include full THMC modeling capabilities. The verification of each individual component of these capabilities can be made against analytical solutions or published numerical solutions. The verification of codes that model coupled processes, however, is more challenging, especially for those involving geomechanics in the suite of coupled processes. Geomechanics stands out in the suite of THMC processes due to the varied numerical approaches adopted to solve rock mechanics problems (e.g, boundary element, discrete fracture, continuum mechanics).

The Geothermal Technologies Office (GTO) was interested in knowing how the various numerical simulators being applied to EGS projects compared. This report documents the comparison of modern computer codes against seven benchmark EGS problems. All seven EGS problems involve the solution of coupled processes from the THMC suite. Twelve teams from universities, national laboratories, and industry participated in the solution of the seven problems, referred to as benchmark problems because of their scale and strict prescriptions of parameters, boundary conditions, domain, and initial conditions. Problems were conceived during an opening workshop at Stanford University in 2013 immediately following the annual Stanford Geothermal Workshop. Problem champions were assigned to each problem, and the problems were finalized after the formal start of the code comparison study in June, 2014. Over the course of the study, problem champions presented their problems and provided initial solutions, and then study participants submitted solutions via a data management system, developed for the project and known as GTO-Velo, which allowed for interactive comparison of results. Solutions were discussed during study teleconferences, and participants were permitted to re-submit solutions or withdraw solutions. Participants were not required to submit solutions for every problem, but were encouraged to submit solutions for problems considered to be somewhat outside the capabilities of the simulator. Solution submissions were closed on May 31, 2015. Solutions recorded in this report are those posted to the GTO-Velo system by May 31, 2015.

This study has demonstrated that while the U.S. EGS simulation community has a diverse set of computational tools with respect to conceptual approaches, they are able to simulate coupled subsurface processes with comparable results, as evidenced by the benchmark problem solutions. The evolution of numerical simulators over the last thirty five years has been impressive, but work remains to be done. Uncertainty in simulation results as measured by the ISO-13528 standard tend to increase with the number of coupled processes in the problem and the modeling of strongly coupled THMC processes remains challenging. The collaborative nature of this study has formed the foundation for the EGS 
simulation community to collectively address field-scale systems, where coupled process modeling will be essential for understanding the system and experimental observations. Confidence in numerical simulation grows from agreement among field experts, especially when diverse perspectives are represented. This study yielded convergence in understanding over the course of each problem via open dialogue and discussions among the participants. 


\section{Acknowledgments}

This material was based upon work supported by the U.S. Department of Energy, Office of Energy Efficiency and Renewable Energy (EERE), Office of Technology Development, Geothermal Technologies Program, under Award Number DE-AC02-05CH11231 with Lawrence Berkeley National Laboratory, under Award Number DE-AC52-07NA27344 with Lawrence Livermore National

Laboratory, and under Award Number DE-AC05-76RL01830 with Pacific Northwest National Laboratory. 



\section{Acronyms and Abbreviations}

ADETL

AD-GPRS

BEM

bgs

CCS

CFRAC

CFRAC-UT

CFD

CFL

$\mathrm{CPU}$

DD

DECOVALEX

DEM

DISAC

DHP

DOE

EERE

EGS

EOS

FALCON

FDM

FEHM

FEM

FLAC

FLAC $^{3 D}$

FVM

GEOS

$\mathrm{GPa}$

GPU

GTO

$\mathrm{HM}$

HPC

IBI

IAPWS

INL
Automatic Differentiation Expression Template Library

Automatic-Differentiation General Purpose Research Simulator

Boundary Element Method

below ground surface

Code Comparison Study

Complex Fracturing ReseArch Code

Complex Fracturing ReseArch Code - The University of Texas at Austin

Computational Fluid Dynamics

Courant Flow Limit

Central Processing Unit

Displacement Discontinuity

DEvelopment of COupled models and their VALidation against EXperiments

Distinct Element Method

Direct Iteration and Successive Approximation Coupler

Downhole Pressure

U.S. Department of Energy

Office of Energy Efficiency and Renewable Energy

Enhanced Geothermal System

Equation of State

FALCON numerical simulator

Finite Difference Method

Finite Element Heat and Mass

Finite Element Method

Fast Lagrangian Analysis of Continua

Fast Lagrangian Analysis of Continua in 3 Dimensions

Finite Volume Method

GEOS advanced supercomputer code

$10^{9}$ Pascals

Graphical Processor Unit

Geothermal Technologies Office

Hydrological-Mechanical

High Performance Computing

Inside Balance Iteration

International Association for the Properties of Water and Steam

Idaho National Laboratory 
IPGT

ISO

$\mathrm{kPa}$

LBNL

LDRD

LLNL

MINC

MOOSE

$\mathrm{MPa}$

NUFT

NTCF

OMP

ORNL

OU

PFLOTRAN

PNNL

PSU

SI

SIF

STOMP

THC

THM

THMC

TOUGH

TR-FLAC3D

TST

UNR

UTA

WHP

XFEM
International Partnership for Geothermal Technology

International Organization for Standarization

$10^{3}$ Pascals

Lawrence Berkeley National Laboratory

Laboratory Directed Research and Development

Lawrence Livermore National Laboratory

Mulitple INteracting Continua

Multiphysics Object Oriented Simulation Environment

$10^{6}$ Pascals

Nonisothermal Unsaturated Flow and Transport

Numerical Transport Code Functionalization

Open Multi-Processing

Oak Ridge National Laboratory

The University of Oklahoma

Parallel FLOw and TRANsport

Pacific Northwest National Laboratory

Pennsylvania State University

Strategic Initiative

Stress Intensity Factor

Subsurface Transport Over Multiple Phases

Thermal-Hydrological-Chemical

Thermal-Hydrological-Mechanical

Thermal-Hydrological-Mechanical-Chemical

Transport Of Unsaturated Groundwater and Heat

TOUGH-REACT-FLAC ${ }^{3 \mathrm{D}}$

Transition State Theory

University of Nevada, Reno

The University of Texas at Austin

Well-Head Pressure

Extended Finite Element Method 


\section{Contents}

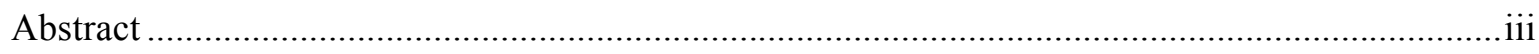

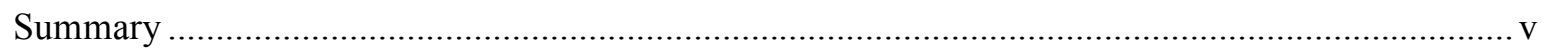

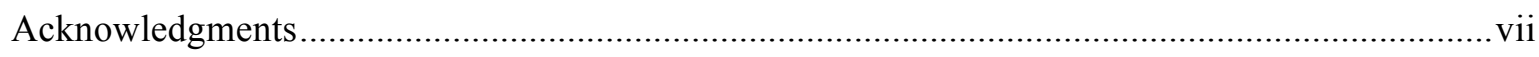

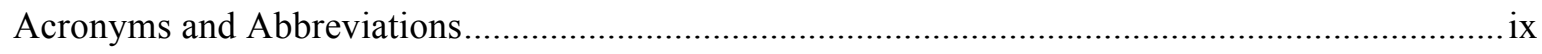

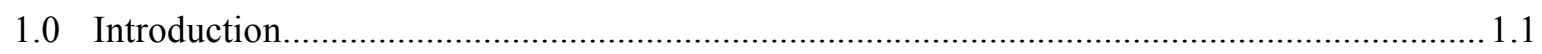

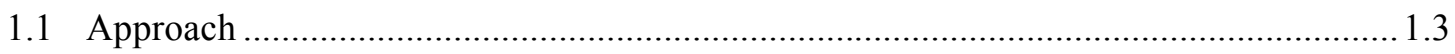

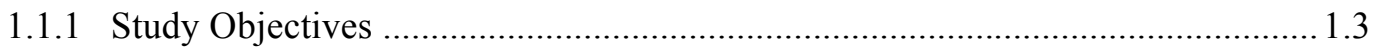

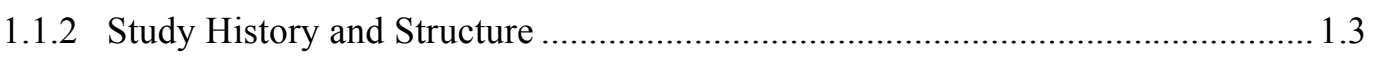

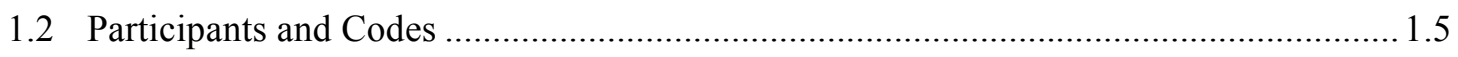

1.3 Benchmark Problems ............................................................................................. 1.9

1.3.1 Benchmark Problem 1: Poroelastic Response in a Fault Zone (PermeabilityPressure Feedback) ........................................................................... 1.9

1.3.2 Benchmark Problem 2: Shear stimulation of randomly oriented fractures aby injection of cold water into a thermo-poro-elastic medium with stress-dependent

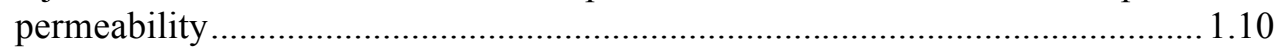

1.3.3 Benchmark Problem 3: Fracture opening and sliding in response to fluid

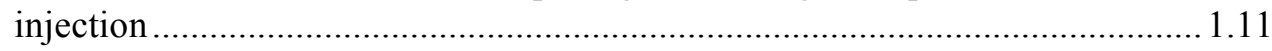

1.3.4 Benchmark Problem 4: Planar EGS fracture of constant extension, pennyshaped or thermo-elastic aperture in impermeable hot rock ............................ 1.12

1.3.5 Benchmark Problem 5: Amorphous Silica dissolution/precipitation in a fracture

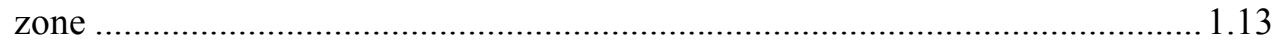

1.3.6 Benchmark Problem 6: Injection into a fault/fracture in thermo-poroelastic rock1.14

1.3.7 Benchmark Problem 7: Surface deformation from a pressurized subsurface

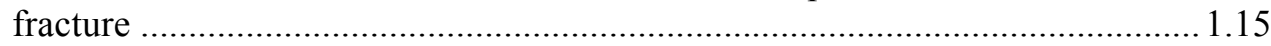

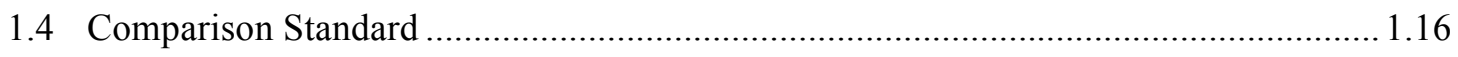

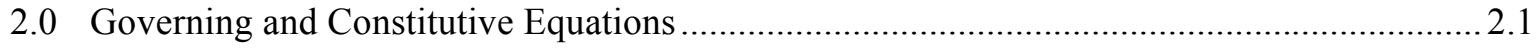

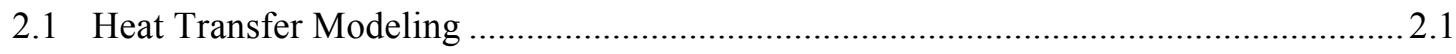

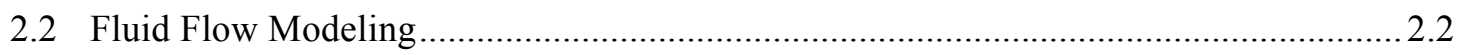

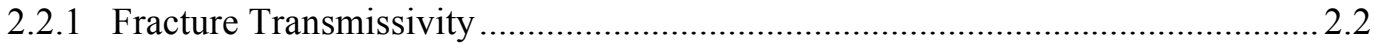

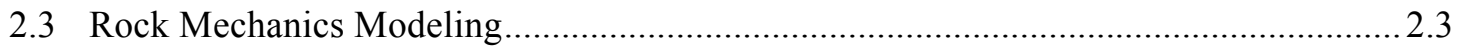

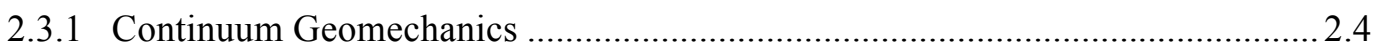

2.3.2 Discrete Fracture Geomechanics ..................................................................... 2.5

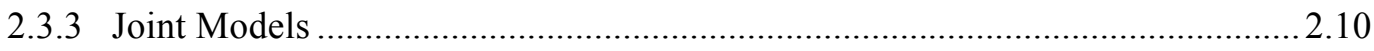

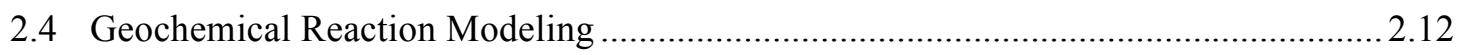

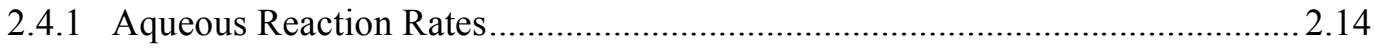

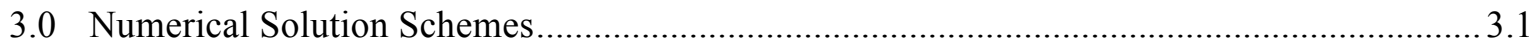

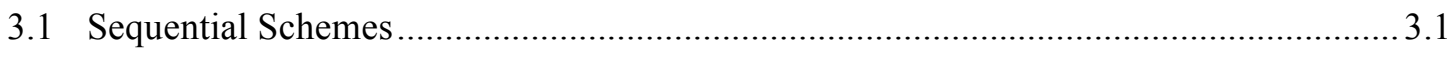

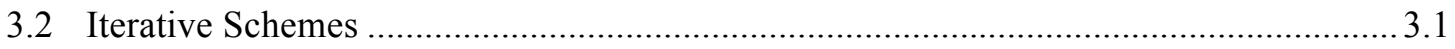


3.2.1 Split Solid and Fracture Mechanics............................................................ 3.1

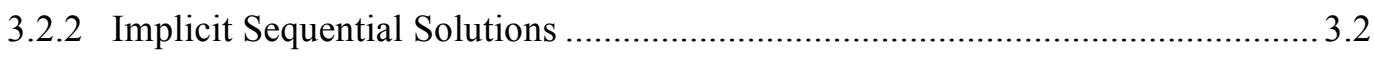

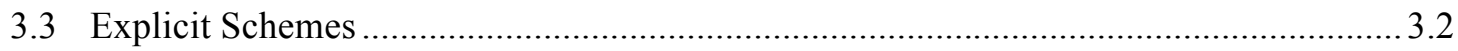

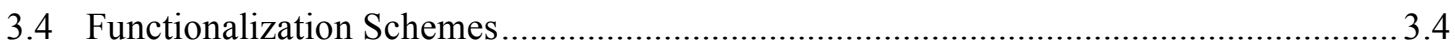

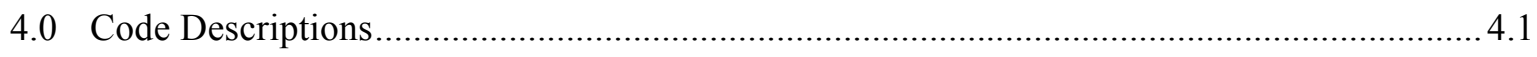

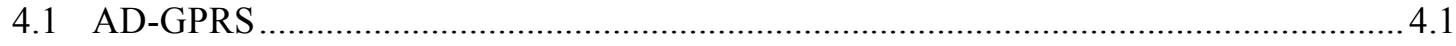

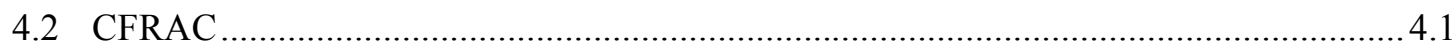

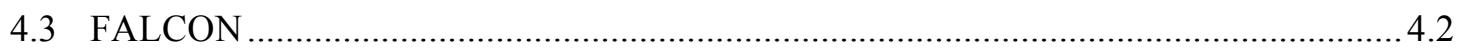

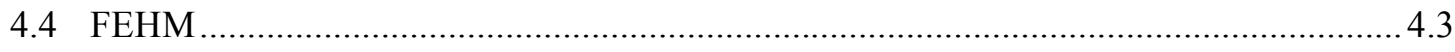

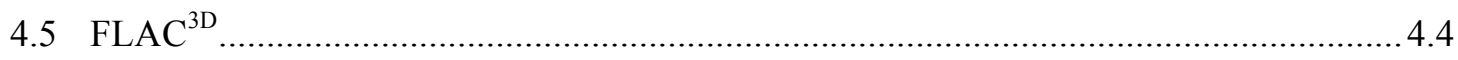

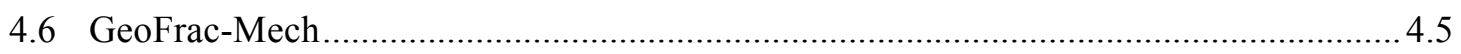

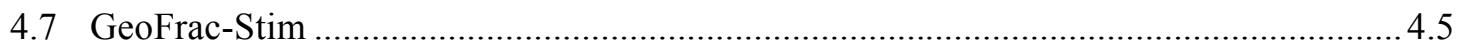

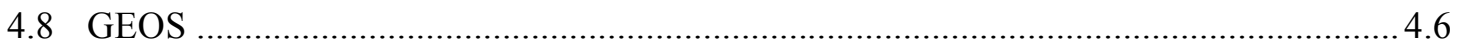

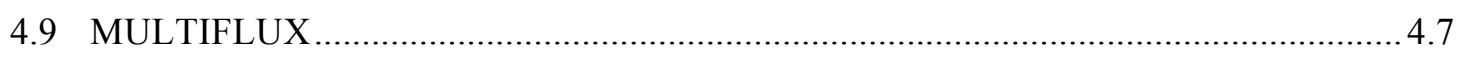

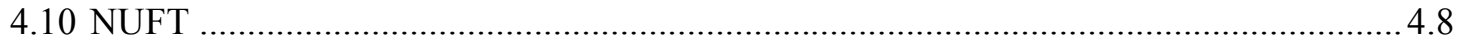

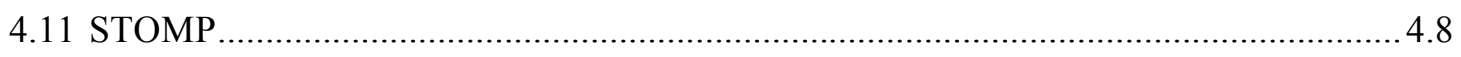

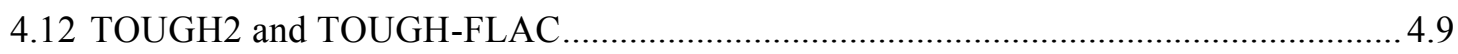

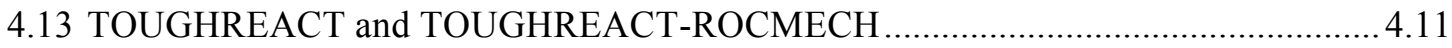

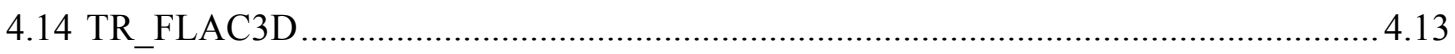

4.15 UDEC Universal Distinct Element Code .................................................................... 4.14

5.0 Benchmark Problem 1: Poroelastic Response in a Fault Zone (Permeability-Pressure

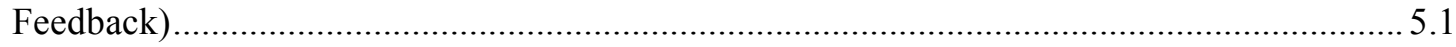

5.1 Geometry and Input Data for Benchmark Problem 1 ............................................... 5.1

5.2 Initial and Boundary Conditions and Sources for Benchmark Problem 1 ..................... 5.3

5.3 Solution Metrics for Benchmark Problem 1 ............................................................... 5.3

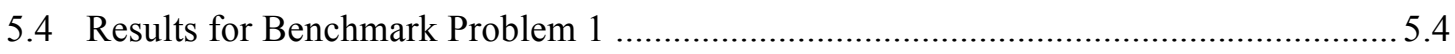

5.5 Discussion of Benchmark Problem 1 Results ............................................................... 5.14

6.0 Benchmark Problem 2: Shear Stimulation of Randomly Oriented Fractures by Injection of Cold Water Into a Thermo-Poro-Elastic Medium with Stress-Dependent Permeability ........ 6.1

6.1 Geometry and Input Data for Benchmark Problem 2 ................................................. 6.2

6.2 Boundary and Initial Conditions and Sources for Benchmark Problem 2 ................... 6.3

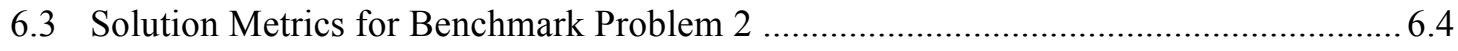

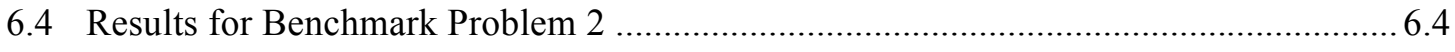

6.5 Discussion of Benchmark Problem 2 Results .......................................................... 6.11

7.0 Benchmark Problem 3: Fracture Opening and Sliding in Response to Fluid Injection........... 7.1

7.1 Geometry and Input Data for Benchmark Problem 3 ................................................ 7.2

7.2 Initial and Boundary Conditions and Sources for Benchmark Problem 3 .................... 7.3

7.3 Physical processes and governing equations for Benchmark Problem 3 ....................... 7.3

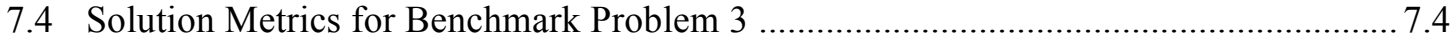




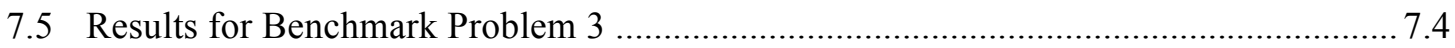

7.6 Discussion of Benchmark Problem 3 Results ............................................................ 7.10

8.0 Benchmark Problem 4: Planar EGS Fracture of Constant Extension and Penny-Shaped or Thermo-elastic Aperture in Impermeable Hot Rock .............................................................. 8.1

8.1 Geometry and Input Data for Benchmark Problem 4 .................................................. 8.1

8.2 Initial and Boundary Conditions and Sources for Benchmark Problem 4 ..................... 8.3

8.3 Solution Metrics for Benchmark Problem 4 ............................................................. 8.7

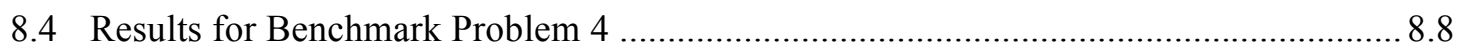

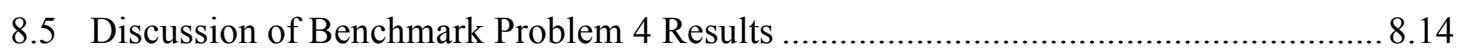

9.0 Benchmark Problem 5: Amorphous Silica Dissolution/Precipitation In A Fracture Zone ..... 9.1

9.1 Geometry and Input Data for Benchmark Problem 5 .............................................. 9.1

9.2 Initial and Boundary Conditions and Sources for Benchmark Problem 5 ..................... 9.3

9.3 Constitutive Relationships for Benchmark Problem 5 .............................................. 9.3

1.1 Reaction Network for Problem 5 ......................................................................... 9.3

9.4 Solution Metrics for Benchmark Problem 5 ............................................................... 9.6

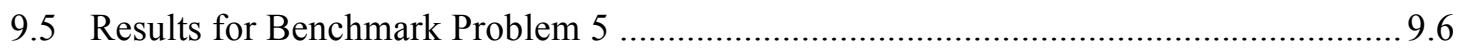

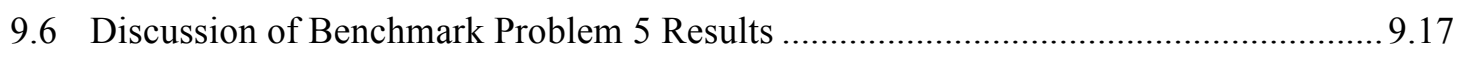

10.0 Benchmark Problem 6: Injection into a Fault/Fracture in Thermo-Poroelastic Rock ........... 10.1

10.1 Geometry and Input Data for Benchmark Problem 6 ............................................. 10.1

10.2 Initial and Boundary Conditions and Sources for Benchmark Problem 6 ................... 10.3

10.3 Solution Metrics for Benchmark Problem 6 ............................................................... 10.3

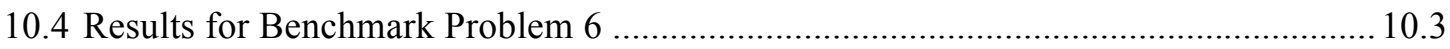

10.5 Discussion of Benchmark Problem 6 Results ............................................................... 10.11

11.0 Benchmark Problem 7: Surface Deformation from a Pressurized Subsurface Fracture ....... 11.1

11.1 Geometry and Input Data for Benchmark Problem 7 ............................................... 11.2

11.2 Initial and Boundary Conditions and Sources for Benchmark Problem 7 ................... 11.3

11.3 Solution Metrics for Benchmark Problem 7 .............................................................. 11.3

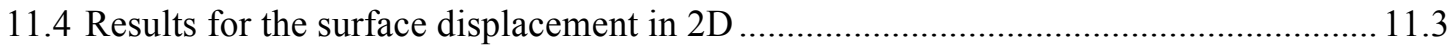

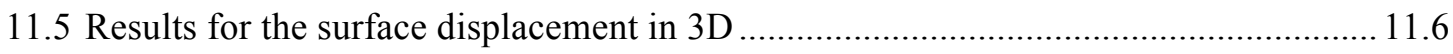

11.6 Comparison of the stress intensity factor results .................................................. 11.10

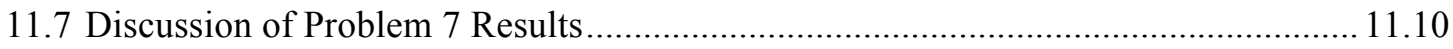

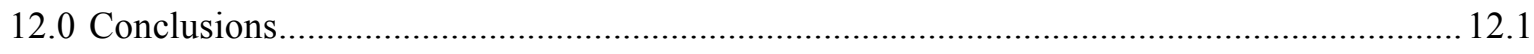

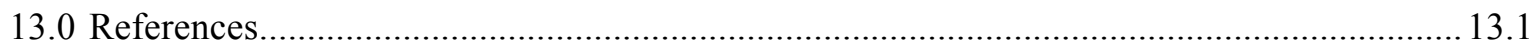




\section{Figures}

Figure 1-1. Schematic representation of Benchmark Problem 1

Figure 1-2. Model domain showing the location of the injection point ...................................... 1.10

Figure 1-3. Problem geometry for the Benchmark Problem 3 ............................................... 1.11

Figure 1-4. Simplified reservoir geometry for Benchmark Problem 4 (Murphy et al. 1981)........ 1.12

Figure 1-5. Idealization of model domain for Benchmark Problem 5 ....................................... 1.13

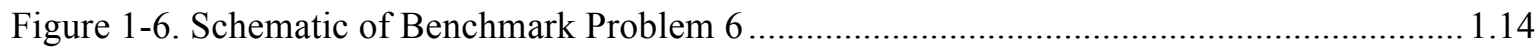

Figure 1-7. Geometry of the fracture in 3D for Benchmark Problem 7 ...................................... 1.15

Figure 4-1. Architecture of the Falcon Code ............................................................................... 4.3

Figure 4-2. Logic flow chart of the new, integrated THMC model ............................................ 4.7

Figure 4-3. Schematic of linking of TOUGH2 with FLAC $^{3 \mathrm{D}}$ for a TOUGH-FLAC coupled THM

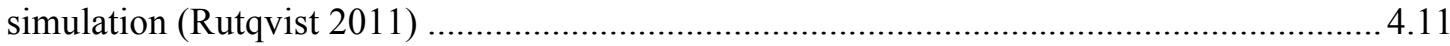

Figure 4-4. The sequential implicit algorithm of chemo-thermo-poro-mechanics. Flow, geomechanics, reactive transport simulators are communicated sequentially (Kim et al. 2015)

Figure 4-5. Coupling relationship between TOUGHREACT, FLAC ${ }^{3 \mathrm{D}}$ and the interpolation module, illustrating the different grids used in the two principal models

Figure 5-1. Conceptual model for Benchmark Problem 1

Figure 5-2 Pressure solution versus time at $\mathrm{r}=14.142 \mathrm{~m}$ for the "no-leak-off" scenario for the 10 teams.

Figure 5-3 Pressure solution versus distance a at $t=10^{4} \mathrm{~s}$ for the "no-leak-off" scenario for the 10 teams

Figure 5-4 Robust average, standard deviation, and ISO 13528 uncertainty in pressure solution versus time at $\mathrm{r}=14.142 \mathrm{~m}$ for the "no-leak-off" scenario for the 10 teams ......................... 5.6

Figure 5-5 Robust average, standard deviation, and ISO 13528 uncertainty in pressure solution versus distance at $\mathrm{t}=10^{4} \mathrm{~s}$ for the "no-leak-off" scenario for the 10 teams

Figure 5-6 Permeability solution versus time at $\mathrm{r}=14.142 \mathrm{~m}$ for the "no-leak-off" scenario for the 10 teams

Figure 5-7. Permeability solution versus distance at $\mathrm{t}=10^{4} \mathrm{~s}$ for the "no-leak-off" scenario for the 10 teams

Figure 5-8. Robust average, standard deviation, and ISO 13528 uncertainty in permeability solution versus time at $\mathrm{r}=14.142 \mathrm{~m}$ for the "no-leak-off" scenario for the 10 teams

Figure 5-9. Robust average, standard deviation, and ISO 13528 uncertainty in permeability solution versus distance at $\mathrm{t}=10^{4} \mathrm{~s}$ for the "no-leak-off" scenario for the 10 teams

Figure 5-10. Pressure solution versus time at $r=14.142 \mathrm{~m}$ for the "leak-off" scenario for the 11 teams

Figure 5-11. Pressure solution versus distance at $\mathrm{t}=10^{4} \mathrm{~s}$ for the "leak-off" scenario for the 11 teams

Figure 5-12. Robust average, standard deviation, and ISO 13528 uncertainty in pressure solution versus time at $r=14.142 \mathrm{~m}$ for the "leak-off" scenario for the 11 teams. 
Figure 5-13. Robust average, standard deviation, and ISO 13528 uncertainty in pressure solution versus distance at $\mathrm{t}=10^{4} \mathrm{~s}$ for the "leak-off" scenario for the 11 teams

Figure 5-14. Permeability solution versus time at $r=14.142 \mathrm{~m}$ for the "leak-off" scenario for the 10 teams

Figure 5-15. Permeability solution versus distance at $\mathrm{t}=10^{4} \mathrm{~s}$ for the "leak-off" scenario for the 10 teams

Figure 5-16. Robust average, standard deviation, and ISO 13528 uncertainty in permeability solution versus time at $\mathrm{r}=14.142 \mathrm{~m}$ for the "leak-off" scenario for the 10 teams...

Figure 5-17. Robust average, standard deviation, and ISO 13528 uncertainty in permeability solution versus distance at $\mathrm{t}=10^{4} \mathrm{~s}$ for the "leak-off" scenario for the 10 teams

Figure 6-1. Observed results of the field stimulation at Desert Peak well 27-15

Figure 6-2. Model domain showing the location of the injection point.

Figure 6-3. Simulation results showing injection rate versus time

Figure 6-4. Permeability calculated by the LANL model at $4.33 \mathrm{~m}$ from the injection point as a function of time.

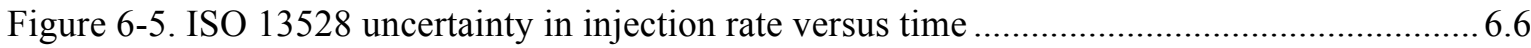

Figure 6-6. Simulation results for temperature versus time at $\mathrm{x}=\mathrm{y}=\mathrm{z}=2.5 \mathrm{~m}$.........................6.7

Figure 6-7. Simulation results for temperature versus time at $x=y=z=7.5 \mathrm{~m}$

Figure 6-8. ISO 13528 uncertainty for temperature versus time at $\mathrm{x}=\mathrm{y}=\mathrm{z}=2.5 \mathrm{~m} \ldots \ldots \ldots \ldots \ldots \ldots . . .6 .8$

Figure 6-9. ISO 13528 uncertainty for temperature versus time at $\mathrm{x}=\mathrm{y}=\mathrm{z}=7.5 \mathrm{~m} \ldots \ldots \ldots \ldots \ldots \ldots . .6 .9$

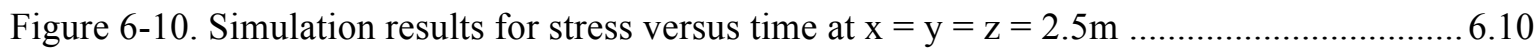

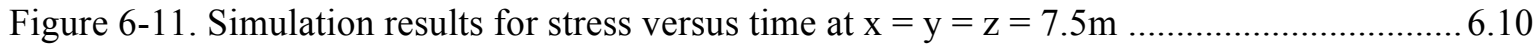

Figure 7-1. Problem geometry for the base case with three preexisting fractures .......................... 7.1

Figure 7-2. Simulation results for injection rate versus time ...................................................... 7.5

Figure 7-3. ISO 13528 uncertainty in injection rate versus time ............................................ 7.5

Figure 7-4. Simulation results for change in fracture aperture (from the initial aperture) after 60

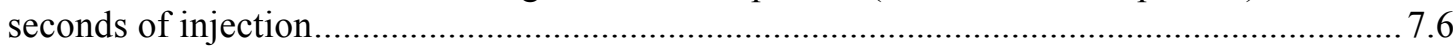

Figure 7-5. ISO 13528 uncertainty in change in fracture aperture after 60 seconds of injection .... 7.6

Figure 7-6. Simulation results for change in fracture aperture (from the initial aperture) at the end of the simulation 7.7

Figure 7-7. ISO 13528 uncertainty in change in fracture aperture at the end of the simulation...... 7.8

Figure 7-8. Simulation results for fracture sliding after 60 seconds of injection. 7.8

Figure 7-9. ISO 13528 uncertainty in fracture sliding after 60 seconds of injection...................... 7.9

Figure 7-10. Simulation results for fracture sliding at the end of the simulation .......................... 7.9

Figure 7-11. ISO 13528 uncertainty for fracture sliding at the end of the simulation .................. 7.10

Figure 8-1. Simplified reservoir geometry (after (Murphy et al. 1981)) ...................................... 8.2

Figure 8-2. Schematic diagram of the near-field and far-field domains of Fenton Hill EGS with a

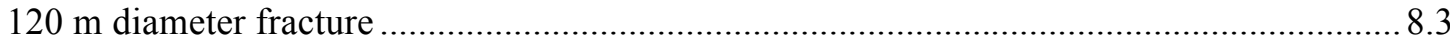

Figure 8-3. Temperature of the rock mass (after (Murphy et al. 1981)) ......................................... 8.4

Figure 8-4. Variation of injection pressure and flow rate during a 75-day test of the Phase I

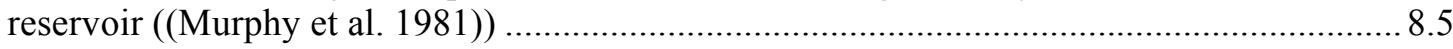


Figure 8-5. Calculated coolant fluid temperature variation with time at the EGS fracture injection point at a depth of $2750 \mathrm{~m}$

Figure 8-6. Coolant fluid back-pressure variation with time at the EGS fracture extraction point at a depth of $2670 \mathrm{~m}$

Figure 8-7. Coolant fluid pressure variation at the EGS fracture injection point at a depth of 2750 $\mathrm{m}$

Figure 8-8. Coolant fluid temperature variation at the EGS fracture extraction point at a depth of $2670 \mathrm{~m}$ (from published data for Fenton Hill).

Figure 8-9. Simulation results for injection pressure versus time for Case 1 with constant aperture8.9

Figure 8-10. Simulation results for injection pressure versus time for Case 2 with variable aperture

Figure 8-11. ISO 13528 uncertainty for injection pressure versus time for Case 1 with constant aperture

Figure 8-12. ISO 13528 uncertainty for injection pressure versus time for Case 2 with variable aperture

Figure 8-13. Simulation results for temperature versus time for Case 1 with constant aperture ... 8.12

Figure 8-14. Simulation results for temperature versus time for Case 2 with variable aperture ... 8.12

Figure 8-15. ISO 13528 uncertainty for temperature versus time for Case 1 with constant aperture8.13

Figure 8-16. ISO 13528 uncertainty for temperature versus time for Case 2 with variable aperture8.13

Figure 9-1. Idealized fracture, altered granite, and unaltered granite zones 9.2

Figure 9-2. Temperature dependence on the coefficients in the B-dot equation..... 9.5

Figure 9-3. Temperature and pressure solutions for the "pure" water scenario from the 3 teams...9.7

Figure 9-4. Permeability and porosity solutions for the "pure" water scenario from the 3 teams...9.7

Figure 9-5. Robust average, standard deviation, and ISO 13528 uncertainty in temperature for the "pure" water scenario.

Figure 9-6. Robust average, standard deviation, and ISO 13528 uncertainty in pressure for the "pure" water scenario.

Figure 9-7. Robust average, standard deviation, and ISO 13528 uncertainty in fracture permeability for the "pure" water scenario

Figure 9-8. Robust average, standard deviation, and ISO 13528 uncertainty in fracture porosity for the "pure" water scenario

Figure 9-9. Temperature and pressure solutions for the "recycle" water scenario from the 3 teams9.10

Figure 9-10. Permeability and porosity solutions for the "recycle" water scenario from the 3 teams

Figure 9-11. Robust average, standard deviation, and ISO 13528 uncertainty in temperature for the "recycle" water scenario

Figure 9-12. Robust average, standard deviation, and ISO 13528 uncertainty in permeability for the "recycle" water scenario

Figure 9-13. Robust average, standard deviation, and ISO 13528 uncertainty in porosity for the "recycle" water scenario

Figure 9-14. Aqueous concentrations (mol/L (aqu)) of $\mathrm{H}+, \mathrm{HSiO}_{-}-$, and $\mathrm{SiO} 2(\mathrm{aq})$ as a function of time at $3.0 \mathrm{~m}$ from the inlet for the "pure" water scenario 
Figure 9-15. Bulk concentrations (mol/L (bulk)) of $\mathrm{SiO} 2(\mathrm{am})$ as a function of time at $3.0 \mathrm{~m}$ from the inlet for the "pure" water scenario

Figure 9-16. Robust average, standard deviation, and ISO 13528 uncertainty in bulk conc. of $\mathrm{SiO} 2(\mathrm{am})$ and aqueous conc. of $\mathrm{SiO} 2(\mathrm{aq})$ for the "pure" water scenario.

Figure 9-17. Aqueous concentrations (mol/L (aqu)) of $\mathrm{H}+, \mathrm{HSiO} 3-$, and $\mathrm{SiO} 2(\mathrm{aq})$ as a function of time at $3.0 \mathrm{~m}$ from the inlet for the "recycle" water scenario.

Figure 9-18. Bulk concentrations (mol/L (bulk)) of $\mathrm{SiO} 2(\mathrm{am})$ as a function of time at $3.0 \mathrm{~m}$ from the inlet for the "recycle" water scenario

Figure 9-19. Robust average, standard deviation, and ISO 13528 uncertainty in bulk conc. of $\mathrm{SiO} 2(\mathrm{am})$ and aqueous conc. of $\mathrm{SiO} 2(\mathrm{aq})$ for the "recycle" water scenario............................ 9.16

Figure 9-20. Temperature dependence of the equilibrium constants 9.18

Figure 9-21. 2-D numerical mesh for Problem 5. Upper - full mesh consisting of 23,750 grid blocks $(\mathrm{NX}=375, \mathrm{NY}=62)$ for the $150 \times 120 \mathrm{~m}$ domain. Lower - enlargement of left corner $(3.0 \times 1.5 \mathrm{~m})$, showing fracture (red), altered granite (yellow), and unaltered granite (gray). The thickess in the Z-direction is $1 \mathrm{~m}$. One large volume grid block was connected to the fracture and altered granite at $\mathrm{X}=0.0$ to enforce the fixed pressure P-T-X boundary condition

Figure 10-1. Fracture orientation and initial stress state; and Mohr-Coulomb failure diagram .... 10.2

Figure 10-2. Simulation results for pressure versus time for the isothermal case in which poroelastic stresses in the matrix rock are considered

Figure 11-1. Geometry of the fracture in 3D and the coordinate systems

Figure 11-2. Vertical surface displacement $\delta_{y}$ normalized by $\delta_{\infty}$ from x $=-4$ a to 4 a for $\beta=0^{\circ}$ for the $2 \mathrm{D}$ case 


\section{Tables}

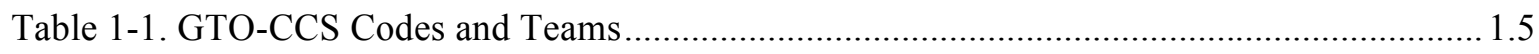

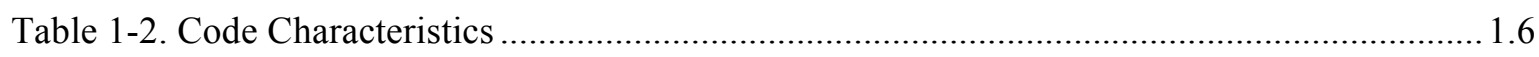

Table 5-1. Participating Teams and Simulators Used in the Solution of Benchmark Problem 1 .... 5.1

Table 5-2. Permeability Function Parameter Values ................................................................. 5.3

Table 5-3. Material Properties .................................................................................................. 5.3

Table 5-4. Comparison Metrics for Benchmark Problem 1 ...........................................................5.4

Table 6-1. Participating Teams and Simulators Used in the Solution of Benchmark Problem 2 .... 6.2

Table 6-2. Material Property Table for Benchmark Problem 2 .................................................. 6.3

Table 7-1. Participating Teams and Simulators Used in the Solution of Benchmark Problem 3 .... 7.2

Table 7-2. Material Properties ............................................................................................. 7.3

Table 8-1. Participating Teams and Simulators Used in the Solution of Benchmark Problem 4 .... 8.1

Table 8-2. Material Property Table for Benchmark Problem 4 ................................................ 8.3

Table 9-1. Participating Teams and Simulators Used in the Solution of Benchmark Problem 5 .... 9.1

Table 9-2. Material Property Table ......................................................................................... 9.2

Table 9-3. Initial mineralogical volume fractions of rock zones ............................................... 9.3

Table 9-4. Equilibrium reactions and coefficients for the temperature-dependent equilibrium

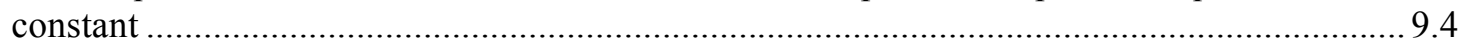

Table 9-5. Kinetic reactions, parameters, and coefficients for the temperature-dependent

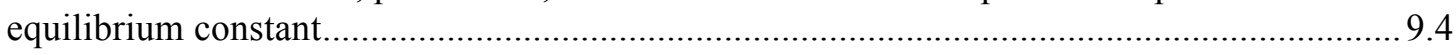

Table 10-1. Participating Teams and Simulators Used in the Solution of Benchmark Problem 610.1

Table 10-2. Material Properties 10.2

Table 11-1. Participating Teams and Simulators Used in the Solution of Benchmark Problem 711.1 Table 11-2. Material Property Table 11.3 


\subsection{Introduction}

Enhanced geothermal systems (EGS) are a promising yet currently under-utilized energy resource (Wood 2009). Extracting energy from geothermal sites can be accomplished in a seemingly straightforward fashion (i.e., drilling a pair of wells to depths where rock temperatures approach $300^{\circ} \mathrm{C}$, fracturing and hydraulically connecting the rock between the wells, and circulating a geofluid from one well to the other through the fractured rock). Potential geofluids are natural brines, compressed $\mathrm{CO}_{2}$, or engineered liquid mixtures, including those with nanoparticles. The geofluid flashes to steam at ambient surface pressures or exchanges heat with a working fluid, which vaporizes during the heat exchange process and subsequently drives an electricity-producing turbine. The cooled geofluid is re-injected into the thermal reservoir directly, or further cooled via secondary heat recovery systems (e.g., building heating). Although conceptually straightforward, like geologic sequestration of greenhouse gases, EGS presents challenges to hydrogeologists to fully realize this energy resource. Mathematical models and numerical simulation are the analytical tools that will be used to help meet these challenges, evaluate the feasibility of EGS at various geothermal sites, and will be essential in designing and evaluating operations of geothermal systems. To establish the credibility of numerical simulators as practical analytical tools, it is essential to demonstrate their capabilities for accurately and reliably modeling EGS processes individually and coupled. A common approach to evaluating numerical simulators, which contributes to their acceptance as practical analytical tools, is to exercise a suite of numerical simulators on problems that consider key processes of interest - to conduct a code comparison study.

Pacific Northwest National Laboratory (PNNL) is supporting the Department of Energy (DOE) Geothermal Technologies Office (GTO) in organizing and executing a geothermal code comparison study (CCS). The purpose of the study is directed at testing and demonstrating modeling capabilities, and also diagnosing differences among those to develop an understanding of the current state of modeling tools available to support geothermal energy developments. The project involves a group of participating teams principally from the United States (U.S.) including those from universities, industry and DOE National Laboratories. $r$ These participating teams generally have unique numerical simulators and analytical approaches, which collectively provide a diverse set of mechanistic approaches, modeled processes and solution schemes for the purposes of this study.. The study design comprises two stages with the participating teams developing solutions for: 1) benchmark problems for the first stage; and 2) challenge problems for the second stage. During the first stage of the study seven benchmark-scale problems were chosen by the participants, with each problem having a champion. Benchmark-scale problems were specifically designed to investigate specific coupled processes typical of enhanced geothermal systems. The plan for the second stage of the study is to undertake problems having laboratory-scale or field-scale observational data. Problem descriptions in the benchmark suite were specific enough to define the required coupled processes to be modeled and the expectation was for numerical simulation results to be comparable between the participating teams. For the challenge problem suite, laboratory or site data will be made available to the participating teams, but the processes to be modeled and the approach for modeling those processes will be the responsibility of each individual team. The benchmark-problem stage of the study was specifically designed to compare numerical simulators under controlled problem conditions; whereas, the challenge-problem stage of the study is designed to investigate the ability of participating teams and numerical simulators to recreate experimental observations.

Numerical simulation of geothermal processes requires the solution of nonlinear equations that describe multifluid flow, heat transport, geomechanics and geochemistry. Early publications on numerical simulations applied to geothermal systems included the works of Mercer and Pinder (Mercer and Pinder 1973), Coats (Coats 1977), and Donaldson and Sorey (Donaldson and Sorey 1979). The Donaldson and Sorey (Donaldson and Sorey 1979) paper noted and discussed differences between numerical simulations and observed data from geothermal fields. In 1980 the results from a geothermal code comparison study 
were published (Stanford Special Panel 1980), as the topic for the panel analysis for the Sixth Annual Workshop in Geothermal Reservoir Engineering. This study involved a suite of six geothermal problems: 1) 1-D Avdonin Solution (C.R. Faust, J.W. Mercer, and W.J. Miller), 2) 1-D Well Test Analysis (M.L. Sorey), 3) 2-D Flow to a Well in a Fracture/Block Media (A.F. Moench), 4) Expanding 2 Phase System with Drainage (M.J. O’Sullivan), 5) Flow in a 2-D Areal Reservoir (J.W. Pritchett), and 6) Flow in a 3-D Reservoir (K. Pruess). Whereas the Stanford 1980 suite of problems considered a variety of geometric configurations and petrophysical property distributions, the principal processes of concern were singlephase flow, two-phase flow, single-phase to two-phase flash, and heat transfer (i.e., TH processes). A total of six numerical simulators were applied to the suite of problems; some simulators were identified by name and others by the developer's institute: 1) Intercomp, 2) Geotrans, 3) Stanford University, 4) Systems, Science and Software $\left.\left(\mathrm{S}^{3}\right), 5\right)$ Lawrence Berkeley Laboratory, and 6) New Zealand Group. Not all problems were attempted by all participating groups. Principal conclusions from the 1980 study were: 1) by supporting the study the U.S. DOE responded rapidly to a relevant industry problem, 2) the problems were well conceived and the reported results demonstrated that the numerical simulators were mathematically sound, and 3) the numerical efficiency of the simulators was an unanswered concern. Two interesting observations from the study were that extensions to the study would be of limited benefit, and that application of the numerical simulators to field sites would only test the ability of the geothermal engineer rather than the simulator.

Regardless of these findings from the Stanford code comparison study (Stanford Special Panel 1980), the role and integration of numerical simulation with respect to geothermal projects has transformed from being supplemental to essential over the last 35 years. Wood (Wood 2009) noted that in spite of the numerous advantageous of EGS technology, a significant determent to its realization is the large upfront capital cost required before producing a single kilowatt of power. Numerical simulation has the potential for reducing the risks and uncertainty associated with the development of advanced geothermal power systems (Fairley et al. 2010). In recognition of the value of numerical simulation in the evolution of EGS, the U.S. DOE, Office of Energy Efficiency and Renewable Energy (EERE) has been supporting recent code development projects (U.S. DOE 2010, 2011, 2015). Over the last twelve years other subsurface science communities have sought to assess the capabilities of numerical simulators for their domainspecific applications via similar code comparison studies. In 2002 a code comparison study was conducted (Pruess et al. 2004) that involved eight test problems addressing $\mathrm{CO}_{2}$ disposal into geologic reservoirs. This effort resulted in valuable insights into the state of numerical simulators in this domain at the time, and provided an assessment of their successful and reliable applications for supporting a new technology development. Conclusions from this study were that results from the participating simulators showed satisfactory agreement on basic problems involving processes that would be induced by $\mathrm{CO}_{2}$ injection into geologic reservoirs. Recognizing the value in such evaluations for tracking and demonstrating the advances in the subject domain, a second $\mathrm{CO}_{2}$ code comparison study (Class et al. 2009) later was conducted with an emphasis on injection scenarios in deep geologic formations. Three problems were developed for this study that focused on 3-dimensional geometries and the developments in numerical simulators since the previous $\mathrm{CO}_{2}$ code comparison study (Pruess et al. 2004). Similarly, a third study considered the scientist/engineer component in conducting numerical simulations (Mukhopadhyaya et al. 2013). For reasons similar to those of the geothermal and carbon sequestration communities, the gas hydrate community conducted an international code comparison study (Anderson et al. 2008; Wilder et al. 2008). A diverse suite conceptual models and numerical solution schemes were applied during this study, yielding good agreement in results for simple problems, but declining agreement between numerical simulators with increasing problem complexity. The field of earthquake rupture dynamics conducted a code verification exercise (Harris et al. 2009) on a series of benchmark problems that have increased in complexity over time. A diverse range of numerical simulators was applied during the study, including those that use finite-element, finite-difference, spectral-boundaryintegral, and spectral-element formulations. For the field of geologic disposal of radioactive waste and spent nuclear fuel, the DECOVALEX project (Hudson and Jing 2013; Tsang et al. 2009) has supported an 
international model comparison collaboration since 1992, principally directed at understanding and modeling coupled thermal-hydrological-mechanical (THM) and thermal-hydrological-mechanicalchemical (THMC) processes in geological systems. A contemporaneous international study focused on reactive transport problems has recently published a description of mathematical and numerical formulations used in modern numerical reactive transport codes for continuum representations of subsurface environments (Steefel et al. 2015). A series of reactive transport benchmark problems were additionally published from this study (Molins et al. 2015; Wanner et al. 2015). While these studies collectively demonstrate the value of coordinated code comparison exercises, they also serve to inspire the project described in this paper.

\subsection{Approach}

\subsubsection{Study Objectives}

The objective of this effort was to create a community forum for Enhanced Geothermal Systems (EGS) reservoir modeling code improvement and verification, building confidence in the suite of available numerical tools, and ultimately identifying critical future development needs for the geothermal modeling community to support the EGS technology development. Numerical simulation is a key method for understanding the creation and evolution of EGS. The development of predictive numerical tools has paralleled roughly four decades of growth of EGS concepts and technology, as well as studies of other unconventional subsurface energy and geologic carbon sequestration settings. These are complex geologic environments where thermodynamics, hydrodynamics, rock mechanics, and geochemistry all contribute critically to system behavior across disparate length and time scales. The usefulness of numerical tools in these settings is moderated by confidence in the quality of the results they produce. Validation with analytical solutions, laboratory and field data, and inter-comparisons with other codes is, therefore, crucial to ensure that simulation can contribute robustly to EGS development.

The principal issues of concern for this project are to determine 1) whether valid mathematical models for the fundamental processes associated with geothermal technologies exist, and 2) whether available numerical simulators assimilate these models to yield reliable and accurate numerical solutions to problems involving conditions of practical interest. The intent for this project is to create a common platform for participants with available numerical simulators to benefit from testing and comparing their codes, diagnosing differences with other codes, and identifying needs in simulation capabilities and additional research. A number of ancillary benefits are also envisioned to result from the study. Importantly, the community-driven nature encourages broad participation and regular interaction across the numerical modeling community, promoting greater awareness and understanding of the capabilities of available tools. The result is a consortium of developers and their codes that includes academic, national laboratory, industry, and international partners. As developers tune their codes to solve benchmark problems, it is also anticipated that they will identify and address new development needs leading to more robust simulators. A collaborative knowledge management system, GTO-Velo (White et al. 2015b) served as a dynamic framework for the participants in this study. A more enduring archive on the U.S. DOE GTO Geothermal Data Repository will be created to document the study, benchmark problems and associated data, and as a library of available codes and their capabilities. These advancements will benefit the existing modeling community, new code developers, and customers of numerical tools alike.

\subsubsection{Study History and Structure}

The code comparison study evolved from a series of discussions held by a Reservoir Modeling Working Group established in the U.S. and through the International Partnership for Geothermal Technologies 
(IPGT). These groups jointly held workshops in March 2010 in Orkugardur, Iceland, and online, with the goal of identifying needs for the next generation of reservoir models to address technical challenges associated with the simulation of EGS and supercritical geothermal systems (Podgorney et al. 2011). These workshops were followed by annual discussions at the May 2010 and May 2011 GTO Peer Review meetings in the Washington, DC area. A common goal identified through this series of meetings was the need to predict and improve the performance of EGS through modeling. Specifically, the modeling community highlighted opportunities to estimate resource lifetime and production potential, manage and mitigate associated environmental issues such as induced seismicity, reduce the cost and risks associated with EGS stimulations by reducing uncertainties, and guide exploration. A new code comparison effort for the geothermal community was identified as a priority towards meeting these goals.

In reaction to the priorities identified by the Reservoir Modeling Working Group and in alignment with DOE goals, GTO engaged PNNL to coordinate the CCS in 2012. The structure and goals for the effort were outlined during a meeting of the working group at the May 2012 GTO Peer Review in Westminster, CO. The following September, the IPGT held a Reservoir Modeling Benchmarking Workshop in Castasegna, Switzerland. There, an international group defined a set of problems that formed a starting point for a GTO Code Comparison problem set. The IPGT defined three categories of test problems: 1) Benchmarks (simple problems for which analytical solutions are known); 2) Test Cases (more complex but well-constrained problems for which there may be no analytical solution but for which all codes are expected to provide comparable solutions, and 3) Challenge Problems (highly complex problems with extensive process coupling, perhaps based on field experimental data, for which the solution is not known a priori and different codes may be expected to provide different solutions).

PNNL and GTO then held a project kickoff workshop in association with the February 2013 Stanford Geothermal Workshop in Palo Alto, CA. The primary goals of the workshop were to 1) introduce the project and its objectives to potential participating team members, and 2) develop an initial set of test problem descriptions for use in the execution stage (Scheibe et al. 2013). The workshop included two breakout sessions during which participants discussed simple to moderate-difficulty benchmark and test cases, and more complex challenge problems. Discussions addressed core code capabilities for EGS simulation, the completeness of the problems identified at the IPGT workshop for meeting CCS goals, metrics for comparison of model outputs, and logistical issues for project implementation. Nine candidate test case problems were identified, along with two classes of challenge problems spanning synthetic and field-based foundations.

During 2013 teams were solicited, problem coordinators identified, problem sets refined from the kickoff workshop results, and participation protocols established. Funded teams were procured in parallel, and the CCS officially began its implementation phase in May 2014. Over the past nine months, participating teams have developed detailed problem descriptions, applied their codes to the selected problems, and submitted simulation results. PNNL has coordinated weekly consortium conference calls and led the development of comparisons of the model outcomes. The effort is underpinned by a web-based knowledge and data management framework for numerical simulation and modeling known as Velo (Gorton et al. 2012), developed at the Pacific Northwest National Laboratory, during both the organization and execution stages of the project and as a portal for the dynamic code comparison archive. The dedicated GTO-Velo platform serves as a framework for participant interaction and project execution. The tool facilitates discussion boards, host's simulator details and problem descriptions, and serves as a repository for associated data, source codes, and other materials needed to maintain an effective study. GTO-Velo is described in more detail by White et al. (White et al. 2015b). 


\subsection{Participants and Codes}

Participation in the code comparison study was solicited by email and announcements at two consecutive Stanford Geothermal Workshops. As discussed above, one-day workshop was held at Stanford University, following the 2013 Stanford Geothermal Workshop, at which the structure of the study was discussed and problems were proposed in two categories: benchmark and challenge (Scheibe et al. 2013). A study kickoff meeting was held during the 2014 Stanford Geothermal Workshop to announce the schedule for the study and introduce participants. Problem champions were assigned to each of the proposed benchmark problems. Problem champions were responsible for developing problem descriptions, submitting initial results, and leading results discussions. Results for the benchmark problems were submitted by 11 teams. Although the study was open to international teams, all participating teams had U.S. affiliations and were from universities, national laboratories, and industry. Team affiliations, members and computer codes are shown in Table 1. The first requirement for all participating teams was to document the codes being applied to the benchmark problems on the GTOVelo code catalog. A synopsis of the code catalog is shown in Table 2, which lists the code name, code developer(s), modeled process classes, key capabilities, spatial and temporal discretization methods or numerical solution approaches, and primary applications. The study schedule was organized around weekly teleconferences/web-conferences. A minimum of three teleconferences/web-conferences were dedicated to each of the benchmark problems: 1) problem description by the problem champion, 2) preliminary result submission and discussions, and 3) final result submission and discussions. Each participating team additionally had the opportunity to present an overview of the computer code(s) they would be applying during the study. A number of the benchmark problems required alterations from their original forms to make them either more specific, to include additional parameters, or to be more approachable across the suite of computer codes. Three of the weekly teleconferences/web-conferences were used to define challenge problems that would be proposed for a sequential study. Participating teams were given the freedom to submit or not submit solutions to the problems, but a diverse suite of solution approaches were sought and encouraged for each problem.

Table 1-1. GTO-CCS Codes and Teams

\begin{tabular}{|c|c|c|}
\hline Code(s) & Team Affiliation & Team Members \\
\hline FALCON & Idaho National Laboratory & $\begin{array}{l}\text { Robert Podgorney, Hai Huang, } \\
\text { Mitch Plummer, Yidong Xia }\end{array}$ \\
\hline FLAC3D & Itasca Consulting Group & $\begin{array}{l}\text { Jason Furtney, Christine Detournay, Azadeh } \\
\text { Riahi, Branko Damjanac }\end{array}$ \\
\hline TOUGH, FLAC3D & $\begin{array}{l}\text { Lawrence Berkeley National } \\
\text { Laboratory }\end{array}$ & $\begin{array}{l}\text { Jonny Rutqvist, Eric Sonnenthal, } \\
\text { Jens Birkholzer }\end{array}$ \\
\hline NUFT, GEOS & $\begin{array}{l}\text { Lawrence Livermore National } \\
\text { Laboratory }\end{array}$ & $\begin{array}{l}\text { Charles Carrigan, Pengcheng Fu, } \\
\text { Bin Guo, Yue Hao, Souheil Ezzedine }\end{array}$ \\
\hline FEHM & Los Alamos National Laboratory & Sharad Kelkar \\
\hline PFLOTRAN & Oak Ridge National Laboratory & Charlotte Barbier, Yarom Polsky \\
\hline $\begin{array}{l}\text { GeoFrac-Mech, } \\
\text { GeoFrac-Stim }\end{array}$ & The University of Oklahoma & $\begin{array}{l}\text { Ahmad Ghassemi, Qinglu Cheng, } \\
\text { Quan Gan, Kai Huang, M.R. Safari, } \\
\text { Varahanaresh Sesetty, Qingfeng Tao }\end{array}$ \\
\hline STOMP & $\begin{array}{l}\text { Pacific Northwest National } \\
\text { Laboratory }\end{array}$ & $\begin{array}{l}\text { Mark White, Signe White, } \\
\text { Diana Bacon, Tim Scheibe }\end{array}$ \\
\hline $\begin{array}{l}\text { TOUGHREACT, } \\
\text { FLAC3D }\end{array}$ & Pennsylvania State University & $\begin{array}{l}\text { Derek Elsworth, Yi Fang, } \\
\text { Kyungjae Im, Baisheng Zheng }\end{array}$ \\
\hline $\begin{array}{l}\text { CFRAC_Stanford, } \\
\text { GPRS }\end{array}$ & Stanford University & Roland Horne, Jack Norbeck, Yang Wong \\
\hline CFRAC_UT & The University of Texas at Austin & Mark McClure, Kit-Kwan Chiu \\
\hline $\begin{array}{l}\text { MULTIFLUX, } \\
\text { TOUGH2, NUFT, 3DEC }\end{array}$ & University of Nevada, Reno & George Danko, Davood Bahrani \\
\hline
\end{tabular}




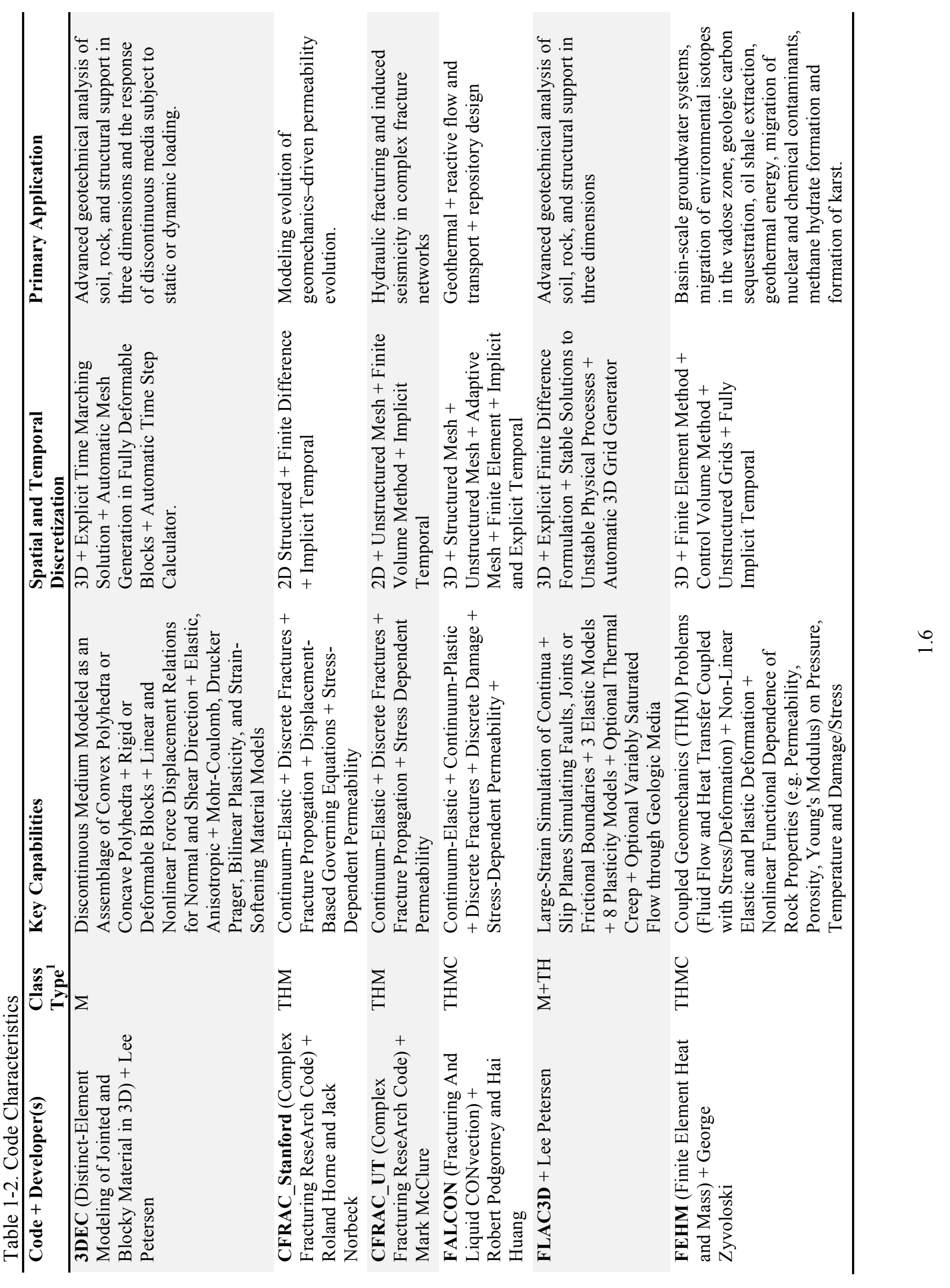




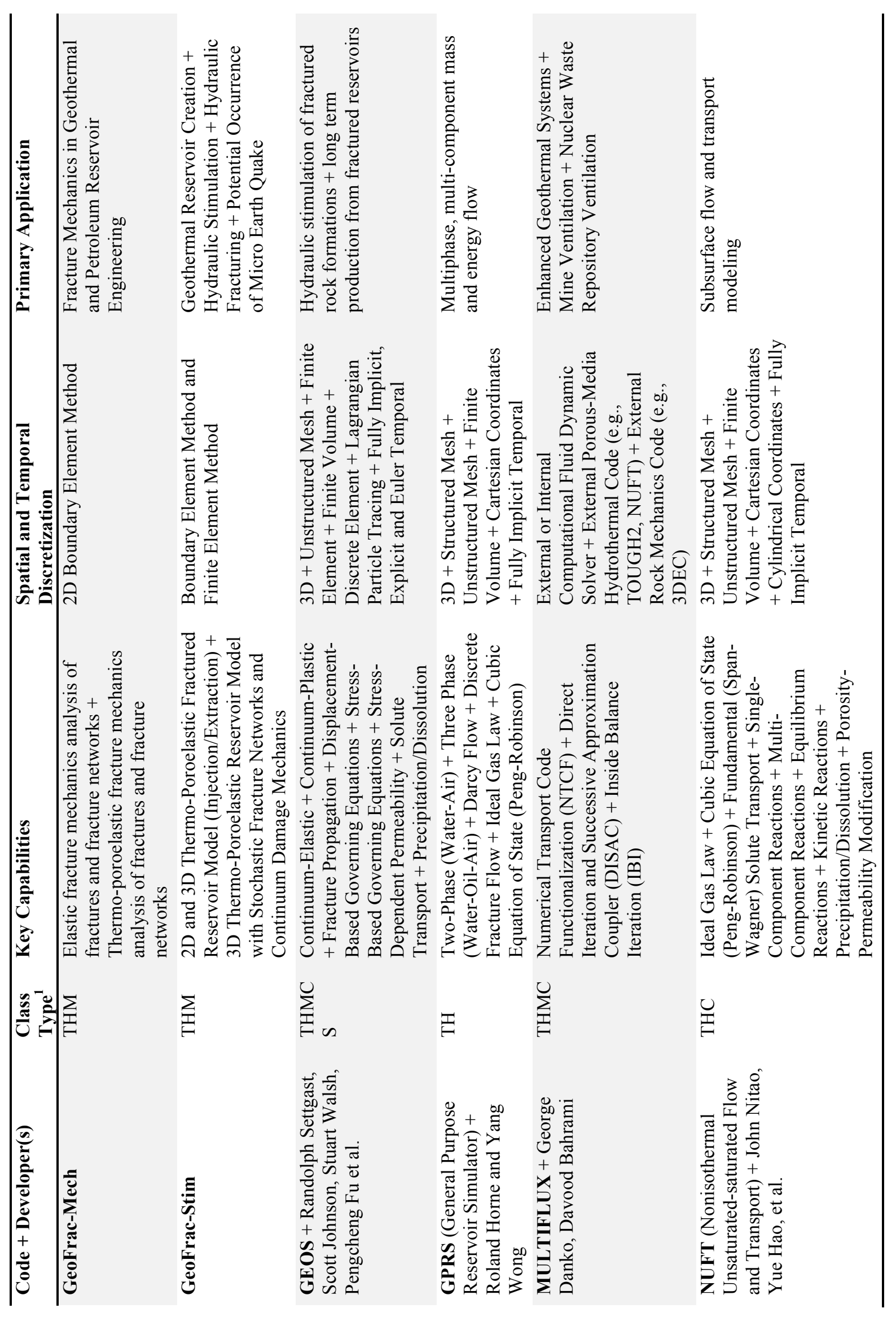

I 


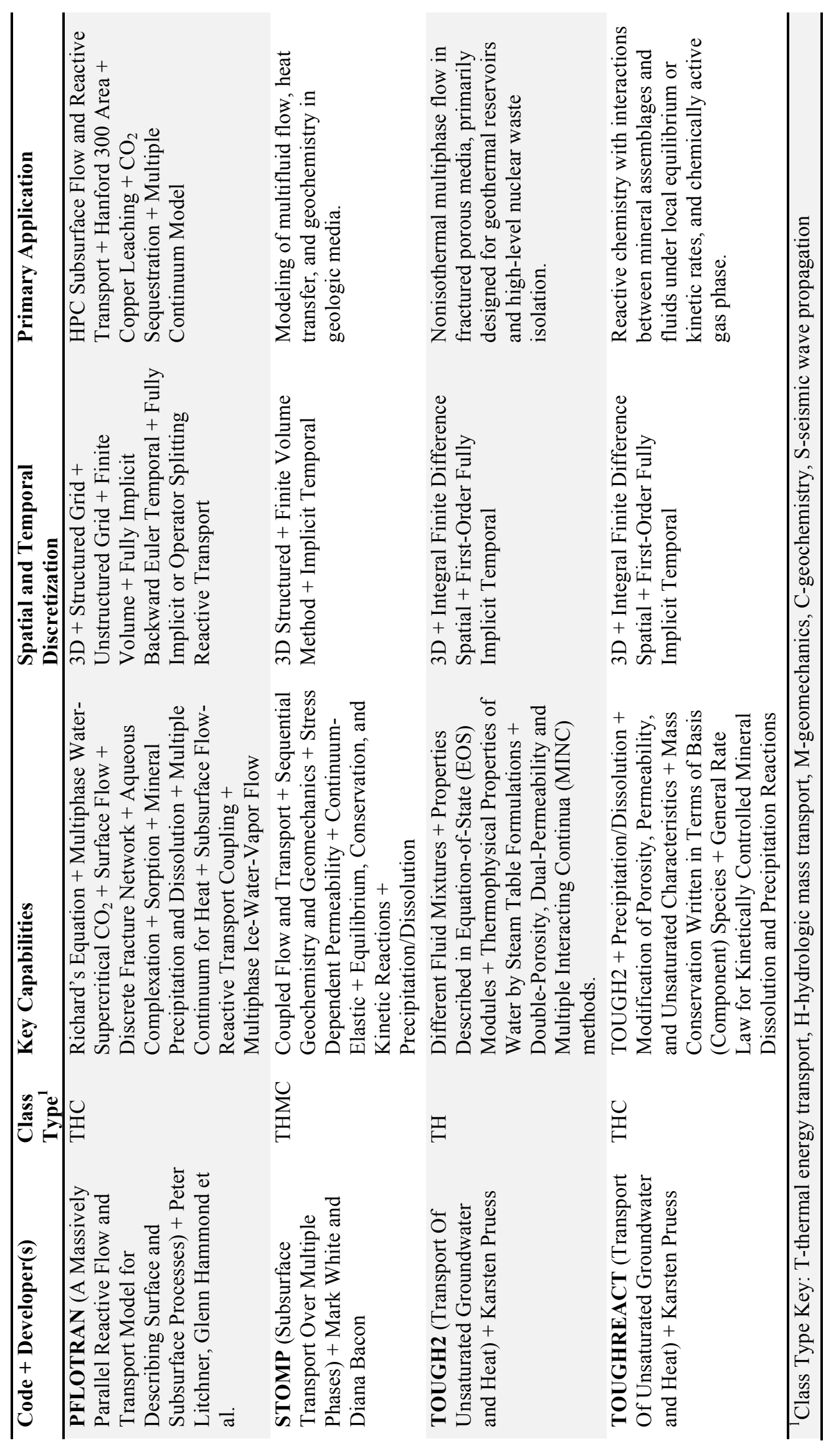




\title{
1.3 Benchmark Problems
}

\subsubsection{Benchmark Problem 1: Poroelastic Response in a Fault Zone (Permeability-Pressure Feedback)}

\author{
Problem Champion: Robert Podgorney, Idaho National Laboratory
}

The first benchmark problem is based on recent observations at a test well from the Raft River EGS demonstration site in Idaho (Bradford et al. 2013; Bradford et al. 2015; Huang et al. 2013), involves a poroelastic fault layer defined as a single fracture in a geothermal reservoir undergoing water injection. Water injected into a fracture within a geothermal reservoir alters the effective stress within the fracture by altering the fluid pressure and formation temperature. This problem considers changes in fracture permeability and injection pressure in response to changes in the effective stress within the fracture (Nathenson 1999). In this problem water is injected from a vertical well into a horizontal fracture with a uniform thickness of $4 \mathrm{~m}$ at a depth of $2,000 \mathrm{~m}$ below ground surface (Fig. 1-1). Water is injected at a

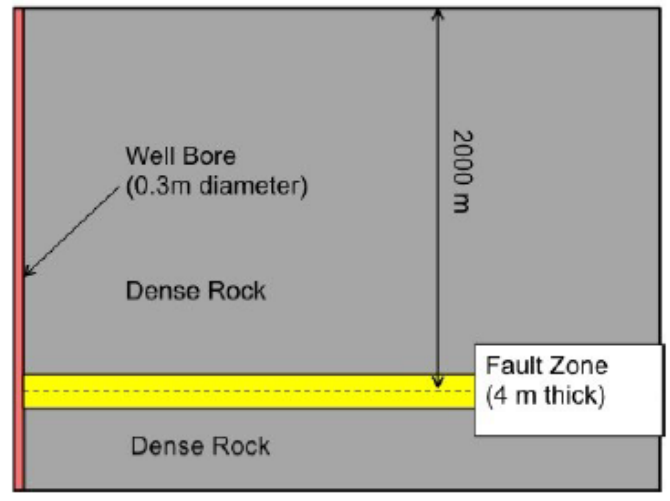

Figure 1-1. Schematic representation of Benchmark Problem 1 constant rate of $80 \mathrm{~kg} / \mathrm{s}$ at a temperature of $140^{\circ} \mathrm{C}$, which is equivalent to the formation temperature, thus eliminating the thermal stress component. The computational domain extends horizontally 2,000 $\mathrm{m}$ (i.e., $2,828.4 \mathrm{~m}$ radially) from the center of the well. Constant pressure conditions are specified at the outer radial distance equivalent to the initial pressure of $20 \mathrm{MPa}$. Two scenarios are considered: 1) no-leak-off and 2) leak-off. In the no-leak-off scenario, the reservoir formation outside of the fracture (i.e., basalt) is assumed to have near zero permeability and in the leak-off scenario the basalt formation has finite permeability. Problem specifications are given and the results are discussed below in Section 5.

Eleven teams submitted results for this problem. Based on the results presented in Section 5, the constant injection rate formulation of this problem leads to a similitude solution during the transient stage of the simulation. Increases in pore pressure decrease the effective stress, leading to an increase in fracture permeability. Simulation results during the transient stage were dependent on the fracture compressibility and basalt formation permeability. The leak-off scenario yielded lower injection pressures and increases in fracture permeability, as injected fluid was lost into the basalt. The original formulation of this problem included a thermal stress component that was affected by injecting cold water into the fracture. The problem was altered to its isothermal form to aid in the initial round of code comparisons. Saturated water equilibrium pressure at $140^{\circ} \mathrm{C}$ is $0.3613 \mathrm{MPa}$, so the reservoir fluid remains in liquid form (i.e., no flash calculations are required for this problem). 


\title{
1.3.2 Benchmark Problem 2: Shear stimulation of randomly oriented fractures aby injection of cold water into a thermo-poro-elastic medium with stress-dependent permeability
}

\author{
Problem Champion: Sharad Kelkar, Los Alamos National Laboratory
}

The second problem was motivated by the shear stimulation treatment of Well 27-15 at the Desert Peak geothermal field in September 2010 (Chabora et al. $2012 \mathrm{~b}$ ). Water at $100^{\circ} \mathrm{C}$ is injected at a specified pressure over a period of 27 days into a threedimensional fractured reservoir via a well with a flowing surface area of $1.131 \mathrm{~m}^{2}$. The initial reservoir temperature was a uniform $190^{\circ} \mathrm{C}$ and the initial fluid pressure in the reservoir at the injection horizon was $9.81 \mathrm{MPa}$. The problem domain was specified to extend horizontally to $200 \mathrm{~m}$ ( $282.4 \mathrm{~m}$ radially) from the injection point. Fluid pressure at the external boundaries was specified as being equal to the initial pressure and the external boundaries were considered to be adiabatic for heat flow. The problem specification used a Cartesian grid for orientation, taking advantage of the $1 / 8$ symmetry, with the injection point being located at the coordinate system origin (i.e., $\mathrm{x}, \mathrm{y}, \mathrm{z}=0,0,0$ ). The downward vertical

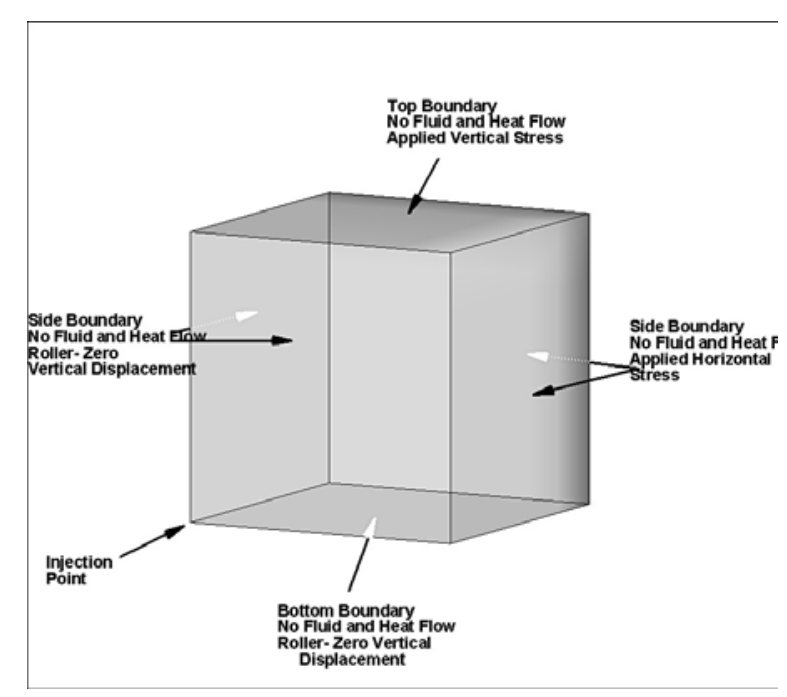

Figure 1-2. Model domain showing the location of the injection point (i.e., negative z-direction) mechanical stress was $22.7 \mathrm{MPa}$, the minimum horizontal stress, applied at the external boundary in the $\mathrm{x}$-direction was $13.88 \mathrm{MPa}$, and the maximum horizontal stress, applied at the external boundary in the y-direction was $18.3 \mathrm{MPa}$. Fluid flow in the reservoir was assumed to occur through randomly oriented fractures, with an isotropic intrinsic permeability that was a function of a Mohr-Coulomb stress, which was defined as a function of the local maximum and minimum principal stresses, the local fluid pressure, and a constant coefficient of friction and cohesion. Permeability was expressed as a linear function of the Mohr-Coulomb stress up to a maximum limit. Problem specifications are given and the results are discussed below in Section 6.

Eight teams submitted results for this problem. Metrics for this problem were the injection rate versus time, and temperature, pressure, and the defined Mohr-Coulomb stress at two distances from the injection point (i.e., $\mathrm{x}=\mathrm{y}=\mathrm{z}=2.5 \mathrm{~m}$ or $\mathrm{r}=4.33 \mathrm{~m}$ and $\mathrm{x}=\mathrm{y}=\mathrm{z}=7.5 \mathrm{~m}$ or $\mathrm{r}=13.0 \mathrm{~m}$ ). The original problem specifications include grid dimensioning, but no reference to an injection surface area. Preliminary results for this problem, conducted by the LBNL team, indicated a strong sensitivity to spatial discretization and the surface area for the well. After the LBNL analysis of the problem, teams were advised to conduct independent grid convergence analysis and the well surface area was specified. As presented in Section 6, agreement of results between teams for this problem was scattered, with similar modeling approaches and grid discretization yielding acceptable agreement in injection rate and temperature, pressure, and displacement at the nearest metric point, but poorer agreement for the second metric point away from the well. Saturated water equilibrium pressure at $190^{\circ} \mathrm{C}$ is $1.254 \mathrm{MPa}$, so the reservoir fluid remains in liquid form (i.e., no flash calculations are required for this problem). 


\subsubsection{Benchmark Problem 3: Fracture opening and sliding in response to fluid injection}

Problem Champion: Mark McClure, The University of Texas at Austin

This isothermal problem involves the injection of fluid into three horizontally connected fractures of finite length. The first fracture is oriented parallel to the maximum principal stress of $\sigma_{\mathrm{yy}}=26 \mathrm{MPa}$ and perpendicular to the minimum principal stress of $\sigma_{\mathrm{xx}}=21 \mathrm{MPa}$ and has a length of $17 \mathrm{~m}$. The second (central) fracture is joined to the end of the first fracture and is oriented at angle of $30.465^{\circ}$ to the maximum principal stress and has a length of $39.446 \mathrm{~m}$. The third fracture is joined to the end of the second, oriented parallel to the maximum principal stress, and has a length of $17 \mathrm{~m}$. Fluid is injected into the center of the second (central) fracture at a constant pressure of $20.25 \mathrm{MPa}$, where the initial fluid pressure was $18 \mathrm{MPa}$. No fluid was allowed to leak from the fractures. The interesting element of this problem is that the

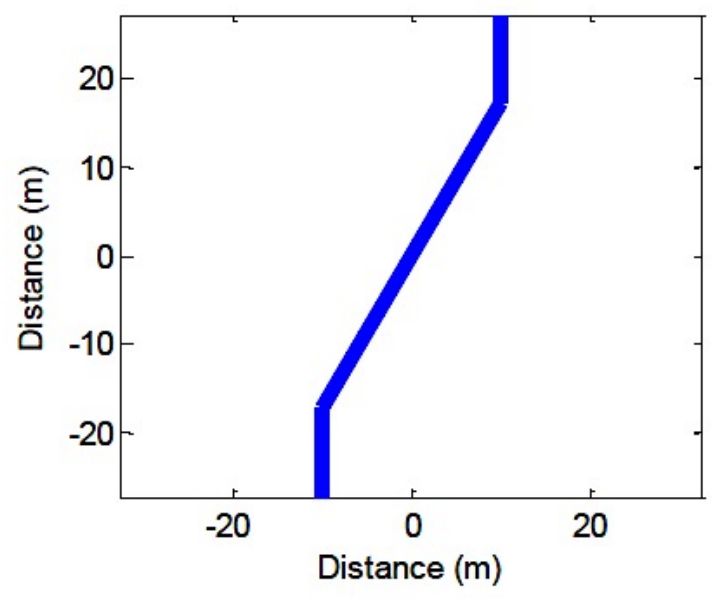

Figure 1-3. Problem geometry for Benchmark Problem 3 injection pressure was selected to be the normal stress on the fracture at all times and for all fracture elements (i.e., $\sigma_{\text {normal }}=21 \mathrm{MPa}$ for the first and third fractures, and $\sigma_{\text {normal }}=22.29 \mathrm{MPa}$ for the second, central, fracture). At the injection pressure, however, the shear stress on the central fracture (i.e., $\sigma_{\text {shear }}=$ $2.186 \mathrm{MPa}$ ) is sufficient to cause slip along the fracture, yielding fracture opening near the first-second fracture joint and second-third fracture joint. Problem specifications are given and the results are discussed below in Section 7.

Seven teams submitted results for this problem. Based on the results presented in Section 7, Fracture opening and slip occurred in response to the fluid injection. Fracture opening occurred via two mechanisms: 1) increase in fluid pressure and 2) shear along the central fracture, yielding fracture opening in the first and third fractures. Fracture opening purely due to the increase in fluid pressure was generally small (i.e., $0.022 \mathrm{~mm}$ ) at equilibrium, however, maximum fracture openings in the first and third fractures near the joints with the second fracture due to slip along the second (central) fracture were more than 30 times that purely due to the increase in fluid pressure. Agreement between the teams for the fracture opening and slip profiles along the length of the fractures was good at equilibrium. More variance was noted during the transient stage of the problem, both in terms of the injection rate and fracture opening and slip profiles. A temperature was not specified for the problem, but the fluid in the fractures is assumed to remain in liquid form with a density of $1000 \mathrm{~kg} / \mathrm{m}^{3}$. 


\title{
1.3.4 Benchmark Problem 4: Planar EGS fracture of constant extension, penny- shaped or thermo-elastic aperture in impermeable hot rock
}

\author{
Problem Champion: George Danko, University of Nevada at Reno
}

The fourth problem was motivated by the first experimental EGS in the U.S. at Fenton Hill (Murphy et al. 1981) and involves a single planar fracture oriented vertically with two slanted wells that intersect the fracture at different elevations. The injection well intersects the fracture at $2750 \mathrm{~m}$ bgs and the production well intersects the fracture at $2630 \mathrm{~m}$ bgs. The fracture has a diameter of $120 \mathrm{~m}$, with the bottom of the fracture being at $2770 \mathrm{~m}$ bgs. This arrangement is an idealization of the configuration at Fenton Hill. Two scenarios are considered for this problem: 1) constant aperture, penny-shaped fracture, and 2) variable aperture, lens-shaped fracture. In the first scenario water flows from the injection well to the production well through the fracture, which has a constant aperture of $0.141 \mathrm{~mm}$. In the second scenario the fracture

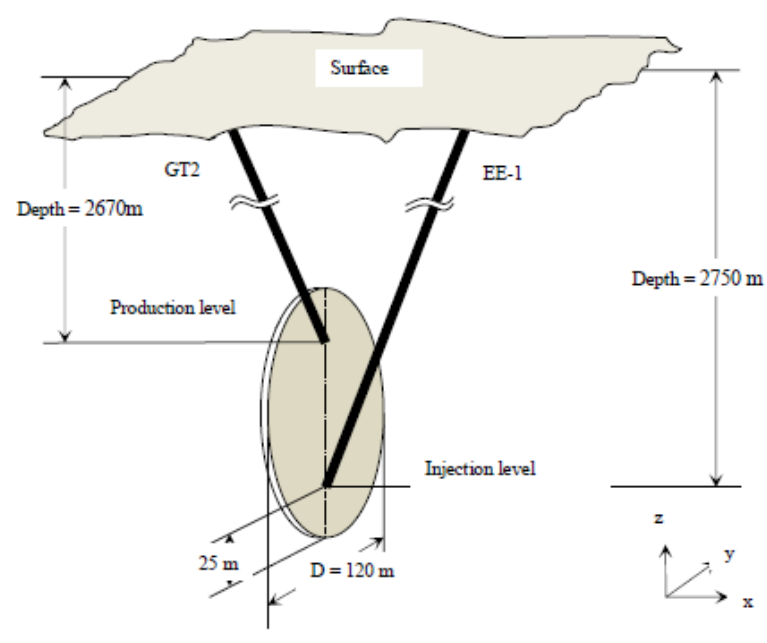

Figure 1-4. Simplified reservoir geometry for Benchmark Problem 4 (Murphy et al. 1981) aperture varies with the elastic and thermal dilatation of the rock, starting with an initial aperture of $1 \mathrm{x}$ $10^{-6} \mathrm{~m}$. The rock temperature at $2750 \mathrm{~m}$ bgs was $185^{\circ} \mathrm{C}$. A geothermal gradient of $100^{\circ} \mathrm{C} / \mathrm{km}$ was assumed from the bottom of the domain until $2300 \mathrm{~m} \mathrm{bgs}$, and then the gradient transitions to $55^{\circ} \mathrm{C} / \mathrm{km}$ until the ground surface. Water is injected at a constant rate of $7.5 \mathrm{~kg} / \mathrm{s}$ for the first 24 days and then at $15.0 \mathrm{~kg} / \mathrm{s}$ between 24 and 75 days. The injection temperature was specified via tabular input as a function of time, designed to be in agreement with a constant surface temperature of $25^{\circ} \mathrm{C}$. Rock matrix properties were isotropic-homogeneous and based on data from Fenton Hill (Murphy et al. 1981). To more accurately model the Fenton Hill field test, outlet pressures were specified as a function of time. Problem specifications are given and the results are discussed below in Section 8.

Six teams submitted results for penny-shaped (i.e., constant aperture) scenario and four teams submitted results for the lens-shaped (i.e., variable aperture) scenario. While pressure results for the constant aperture scenario show similar trends among the team results, they vary in magnitude; moreover, none of the pressure results match the trends observed at Fenton Hill. At Fenton Hill measured pressure values also increased in response to the jumps in inject rate; however, the magnitude of the measured increase was significantly lower than the predicted results. Furthermore, the measured data shows pressure decline over time at the field with constant injection, indicating further fracture opening in the system. Submitted simulation results for temperature showed generally good agreement among the teams and reasonable agreement with the outlet temperature history observed at Fenton Hill. As the injection rate is specified for this problem, the agreement between simulation results in terms of temperature indicates all of the simulators are modeling fluid enthalpy and heat transfer processes correctly. Pressure results from three of the participating teams showed similar trends capturing the measured behavior with varying magnitudes The results from these three teams did yield declining pressures, following sharp pressure increases with jumps in the injection rate. 


\title{
1.3.5 Benchmark Problem 5: Amorphous Silica dissolution/precipitation in a fracture zone
}

\author{
Problem Champion: Mark White, Pacific Northwest National Laboratory
}

For this problem, flow of water through an idealized horizontal fracture zone is considered with temperature dependent reaction of the water with a formation mineral. This problem is an altered version of the simulations conducted by $\mathrm{Xu}$ and Pruess (Xu et al. 2004a) that investigated the effects of mineral scaling and clay swelling in a fractured geothermal reservoir. The temperature dependent amorphous silica reaction kinetics follow the general form of the rate law (Steefel and Lasaga 1994). The fracture zone comprises three regions: 1) fracture, 2) altered granite, and 3) unaltered granite. The flow of water is predominately through the fracture, but the altered granite and unaltered granite have finite intrinsic

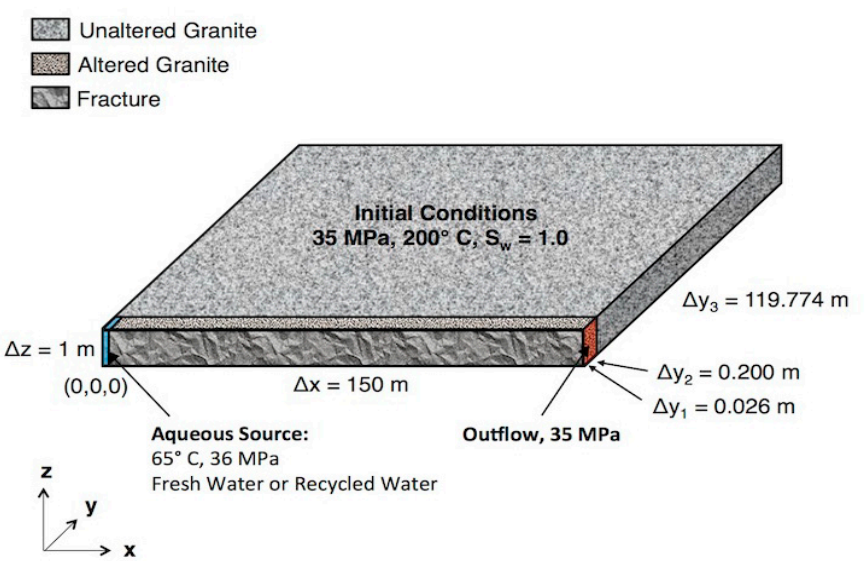

Not to scale

Figure 1-5. Idealization of model domain for Benchmark Problem 5 permeability and porosity. Water at $65^{\circ} \mathrm{C}$ is injected into the fracture zone, which is initially at $200^{\circ} \mathrm{C}$. The dissolution and precipitation of amorphous silica along the length of the fracture zone is to be computed for two forms of injected water: 1) pure water and 2) recycled water. Changes in intrinsic permeability and porosity due to mineral dissolution and precipitation are also considered. A relationship between changes in porosity and intrinsic permeability developed by Verma and Pruess (Verma and Pruess 1988) is used to more accurately capture the effect of pore-throat clogging by precipitates. Metrics for this problem were profiles of temperature, pressure, porosity, and permeability within the fracture across the domain at 10 years; aqueous concentrations of $\mathrm{H}+, \mathrm{OH}-, \mathrm{HSiO} 3-$, and $\mathrm{SiO} 2$ (aq) as a function of time at $3.0 \mathrm{~m}$ from the inlet; and change in amorphous silica abundance as a function of time at $3.0 \mathrm{~m}$ from the inlet. Problem specifications are given and the results are discussed below in Section 9.

Three teams submitted results for this geochemical problem using THC models. For the fresh water scenario, all simulation results showed general agreement in the temperature, pressure, porosity, and permeability profiles at 10 years. The pressure profile showed a nearly linear decay, with a slower decay rate near the inlet, due to the dissolution of amorphous silica. Sharp decreases in porosity and permeability were additionally noted in all the simulation results near the inlet, indicating the inlet water was saturated with aqueous species at the outlet. For the recycle-water scenario, the problem is designed to have supersaturated conditions for dissolved aqueous species at the inlet. This results in precipitation of amorphous silica near the inlet. All the simulation results show decreases in porosity and permeability near the inlet for this scenario after 10 years, indicating precipitation of amorphous silica. Comparison of the aqueous species concentrations and change in amorphous silica abundance at $3.0 \mathrm{~m}$ from the inlet between the submitted simulation results agree with the changes in porosity and permeability, indicating proper modeling of the equilibrium reactions. 


\subsubsection{Benchmark Problem 6: Injection into a fault/fracture in thermo- poroelastic rock}

Problem Champion: Ahmad Ghassemi, The University of Oklahoma

The principal objective of this problem is to illustrate the role of coupled thermo-poroelastic processes on natural fracture opening and shear deformation, which develops upon water injection into the fracture. The problem is two-dimensional and considers a 40-m long fracture oriented at $45^{\circ}$ to the principal horizontal stress directions; where, the minimum principal stress is $13 \mathrm{MPa}$ and the maximum principal stress is 20 $\mathrm{MPa}$. The original problem specified an initial pore pressure, which was not in equilibrium with the mechanical stress state. The final form of the problem starts under equilibrium conditions with the principal stresses, initial pore pressure of $10 \mathrm{MPa}$, temperature of $420^{\circ} \mathrm{K}$, and an aperture of $1 \mathrm{~mm}$. Water at $420^{\circ} \mathrm{K}$

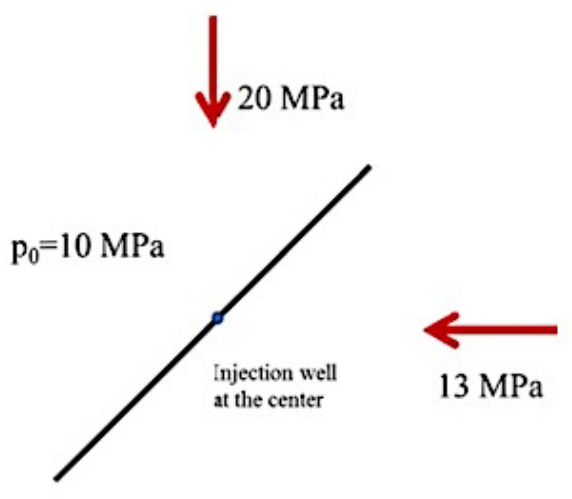
(isothermal) or $400^{\circ} \mathrm{K}$ (nonisothermal) is injected into this reservoir at a constant rate of $0.6 \times 10^{-7} \mathrm{~m}^{3} / \mathrm{s}$ per meter thickness of reservoir. The rock matrix properties are modeled after a Westerly granite. The problem is designed such that water injection into the fracture yields opening and shear. The shear strength of the fracture is modeled using the Bandis-Barton model (Bandis et al. 1983; Barton et al. 1985). The fracture normal stiffness is modeled following the Bandis (Bandis et al. 1983) joint closure function with a specified joint normal stiffness of $0.5 \mathrm{GPa} / \mathrm{m}$ and maximum joint closure of $3.0 \mathrm{~mm}$. Metrics for the problem include reporting of the fluid pressure, fracture aperture, and fracture shear as a function of time; and profiles of fracture aperture and shear along the fracture length at 5, 75, and 180 days after the start of water injection. Problem specifications are given and the results are discussed below in Section 10. 


\title{
1.3.7 Benchmark Problem 7: Surface deformation from a pressurized subsurface fracture
}

\author{
Problem Champion: Pengcheng Fu, Lawrence Livermore National Laboratory
}

This problem is largely based on the work of Pollard and Holzhausen (Pollard and Holzhausen 1979) and entails the calculation of ground surface deformation in response to fluid injection into a subsurface fracture. The fluid pressure is assumed to act uniformly over the fracture surfaces and the fracture is idealized as being rectangular in shape, oriented at various dipping angles. The rock matrix is assumed to have isotropic-homogeneous properties and the mechanical characteristics are to be modeled as being linear elastic. The problem was posed in both 2- and 3-dimensional forms. In 3dimensional form the fracture is specified as being $2 \mathrm{a}$ wide and $2 \mathrm{~b}$ long, with the coordinate system centroid being located on the ground surface, vertically (y-direction) directly above the centroid of the fracture. The z-direction coordinate system axis is oriented with the length of the fracture and the $\mathrm{x}$-direction is oriented with dipping or width direction. The dipping angle is respect to a horizontal axis. The in situ stress is assumed to be isotropic and the specified net fluid pressure is the difference between the normal in situ stress on the fracture and the actual

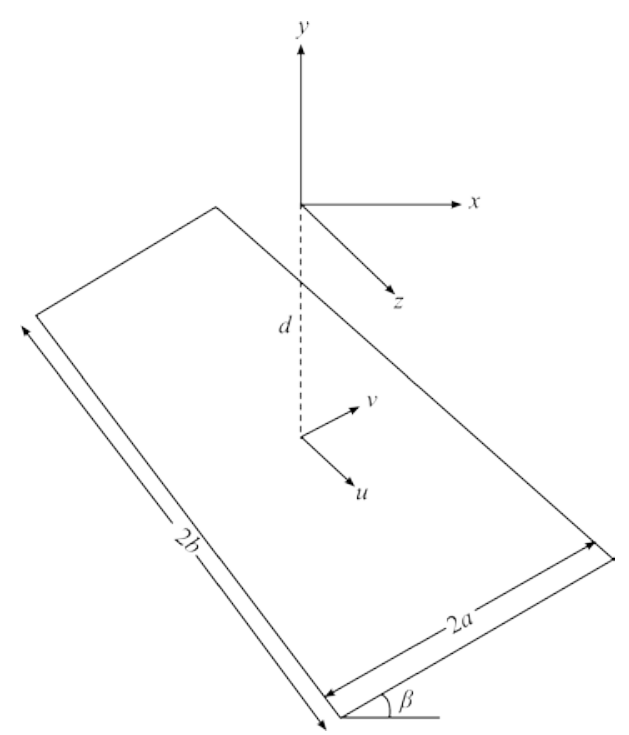

Figure 1-7. Geometry of the fracture in 3D for Benchmark Problem 7 fluid pressure. A static solution to the problem is sought, ignoring the transients. Metrics for the problem are the vertical surface displacements from $\mathrm{x}=-4 \mathrm{a}$ to $\mathrm{x}=4 \mathrm{a}$ for dipping angles of $0^{\circ}, 45^{\circ}$, and $90^{\circ}$. Additional metrics are the mode-I and mode-II stress intensity factors at the two fracture tips. Problem specifications are given and the results are discussed below in Section 11.

Six teams submitted results for this problem, three teams submitting both $2 \mathrm{D}$ and $3 \mathrm{D}$ results, two teams submitting only 2D results, and one team submitting only 3D results. The LLNL team conducted a suite of simulations for the 2D scenario to determine the sensitivity of simulation results of surface vertical displacement to the mesh resolution and grid element types. They noted that for meshes with element lengths less than $0.2 \mathrm{a}$, the results are not very sensitive to mesh resolution and that quadrilateral and triangular grid elements yielded comparable results. Simulation results submitted for the 2D scenarios show good agreement, with the flat fracture $\left(\beta=0^{\circ}\right)$ yielding the greatest surface displacements and the vertical fracture $\left(\beta=90^{\circ}\right)$ yielding a symmetrical double peaked surface profile, with negative surface displacements near the center and peak fringes. More diverse results were submitted for the $3 \mathrm{D}$ scenarios, but agreement between the teams is considered to be acceptable. 


\subsection{Comparison Standard}

Advances in numerical simulation capabilities in terms of modeling coupled processes, heterogeneous environments, time varying driving forces, and computational domains, makes comparison of numerical simulators against analytical solutions more and more difficult. The alternatives for EGS are comparisons against controlled laboratory-, meso-, or field-scale experiments, comparison against field operations, or comparisons among numerical simulators. One difficulty with code comparison studies is the absence of a true value for the target quantity (e.g., pressure, flow rate, fracture permeability, shear displacement). The use of the ISO-13528 standard, developed by the chemical and physical measurement communities to proficiency test computer simulation for canopy reflectance models, is reported by Widlowski et al. (Widlowski et al. 2013). The central concept followed is to use a surrogate for the unknown true value of a target quantity, referred to as the robust average, and a robust standard deviation of the collective numerical simulation results using a robust algorithm (ISO 13528 2005).

The algorithm (ISO 13528 2005) to compute the robust average and robust standard deviation is iterative. Consider a simulation whose output of interest is the pressure at a location within a three-dimensional domain over time. The algorithm is initialized by estimating the robust average and robust standard deviation

$$
\begin{aligned}
& \bar{P}^{*}=\text { median of } P_{i}(i=1,2, \ldots, N) \\
& s_{P}^{*}=1.483 \text { median of }\left|P_{i}-\bar{P}^{*}\right|
\end{aligned}
$$

where, $P_{i}$ is the pressure solution from each team. Updates to the robust average and robust standard deviation are then computed in an iterative process

$$
\begin{aligned}
& \delta=1.5 s_{P}^{*} \\
& P_{i}^{*}=\left\{\begin{array}{c}
\bar{P}^{*}-\delta \text { if } P_{i}<\bar{P}^{*}-\delta \\
\bar{P}^{*}+\delta \text { if } P_{i}>\bar{P}^{*}+\delta \\
P_{i}
\end{array}\right. \\
& \bar{P}^{*}=\frac{1}{N} \sum_{i}^{N} P_{i}^{*} \\
& s_{P}^{*}=1.134 \sqrt{\frac{1}{N-1} \sum_{i}^{N}\left(P_{i}^{*}-\bar{P}^{*}\right)}
\end{aligned}
$$

Convergence was determined by a stable robust average pressure

$$
\frac{\Delta \bar{P}^{*}}{\bar{P}^{*}}\left(\Delta \bar{P}^{*} / \bar{P}^{*}\right)<10^{-6}
$$

The standard uncertainty of the robust mean was then estimated as

$$
u_{P}=1.25 s_{P}^{*} / \sqrt{N}
$$


where according to the ISO 13528 (2005), the factor 1.25 represents the ratio of the standard deviation of the median to the standard deviation of the arithmetic mean, for large samples (i.e., $\mathrm{N}>10$ ) from a normal distribution. For data not normally distributed Eqn. (4) yields a value between the standard deviation of the arithmetic mean and standard deviation of the median, yielding a conservative estimate of the standard uncertainty. 



\subsection{Governing and Constitutive Equations}

\subsection{Heat Transfer Modeling}

Heat transfer in EGS is generally considered to be via thermal conduction and fluid advection. Radiation heat transfer is largely ignored in the modeling of heat transfer in the subsurface for EGS. The governing equation for heat transfer equates the change in internal energy over time of a volume of geologic media, including fluids in the pore space, with the flux of thermal energy into the volume over the surface of the volume via thermal conduction and advection, plus source rates of thermal energy and fluid mass into the control volume:

$$
\begin{aligned}
& \frac{\partial}{\partial t} \int_{V_{n}}\left[\sum_{\gamma=l, g}\left(\phi \rho_{\gamma} s_{\gamma} u_{\gamma}\right)+\left((1-\phi) \rho_{r} u_{r}\right)\right] d V_{n}=\int_{\Gamma_{n}}[\mathbf{E} \cdot \mathbf{n}] d \Gamma_{n} \\
& +\int_{V_{n}}\left[\sum_{\gamma=l, g}\left(h_{\gamma} m_{\gamma}\right)+q_{e}\right] d V_{n}
\end{aligned}
$$

where, $t$ is time (s), $V_{n}$ is the volume (m), $\phi$ is the porosity, $\rho_{\gamma}$ is the fluid density $\left(\mathrm{kg} / \mathrm{m}^{3}\right), s_{\gamma}$ is the fluid saturation, $u_{\gamma}$ is the fluid internal energy $(\mathrm{J} / \mathrm{kg}), \rho_{r}$ is rock density $\left(\mathrm{kg} / \mathrm{m}^{3}\right), u_{r}$ is the rock internal energy $(\mathrm{J} / \mathrm{kg}), \mathbf{E}$ is the energy flux tensor $\left(\mathrm{W} / \mathrm{m}^{2}\right), \mathbf{n}$ is the unit surface normal vector, $\Gamma_{n}$ is the volume surface area $\left(\mathrm{m}^{2}\right), h_{\gamma}$ is the enthalpy of the fluid $(\mathrm{J} / \mathrm{kg}), m_{\gamma}$ is the mass rate into the volume $(\mathrm{kg} / \mathrm{s})$, and $q_{e}$ is the thermal energy source into the volume (W). Energy flux into the control volume is via thermal conduction and fluid advection:

$$
\mathbf{E}=-\nabla\left(\mathbf{k}_{e} \nabla T\right)+\sum_{\gamma=l, g}\left(\rho_{\gamma} h_{\gamma} \mathbf{V}_{\gamma}\right)
$$

where, $\mathbf{k}_{e}$ is the effective thermal conductivity tensor $(\mathrm{W} / \mathrm{m} \mathrm{K}), T$ is the temperature $\left({ }^{\circ} \mathrm{C}\right)$, and $\mathbf{V}_{\gamma}$ is the fluid flux tensor volume $\left(\mathrm{kg} / \mathrm{s} \mathrm{m}^{2}\right)$. Fluid flux is generally assumed to follow Darcy's law, avoiding the need for a momentum balance equation:

$$
\mathbf{V}_{\gamma}=-\frac{k_{r \gamma} \mathbf{k}}{\mu_{\gamma}}\left(\nabla P_{\gamma}+\rho_{\gamma} g \mathbf{z}\right)
$$

where, $k_{r \gamma}$ is the fluid relative permeability, $\mathbf{k}$ is the intrinsic permeability tensor $\left(\mathrm{m}^{2}\right), \mu_{\gamma}$ is the fluid viscosity (Pa s), $P_{\gamma}$ is the fluid pressure (Pa), $g$ is the acceleration of gravity $\left(\mathrm{m} / \mathrm{s}^{2}\right)$, and $\mathbf{z}$ is the gravitation unit vector. The benchmark problems of this study only considered an aqueous fluid, but more general situations for EGS include gases and aqueous fluids. The general practice in solving the conservation of energy equation is to ignore the off-diagonal tensor values. Density, internal energy, enthalpy, viscosity, and thermal conductivity of the fluids are typically considered to be functions of fluid composition, temperature and pressure. For the aqueous fluid, the founding formulations are those of pure water, which are generally adopted from the International Association for the Properties of Water and 
Steam (IAPWS) (Wagner et al. 2000). The range of validity for the IAPWS formulations are temperatures between 273.15 and 1073.15 and pressures below $100 \mathrm{MPa}$, which is sufficient for EGS applications.

\subsection{Fluid Flow Modeling}

Fluid flow outside of geologic media is often solved via the Navier-Stokes momentum equation, coupled with a conservation of mass equation, and associated constitutive equations for the fluid properties. Fluid flow in geologic media typically takes advantage of Darcy's equations, scaling viscous losses with pressure gradient and eliminating the need to solve the Navier-Stokes momentum equation. For EGS, fluid flow is often dominated by discrete fractures or fracture systems, but may also involve flow through the rock matrix. A principal assumption taken in the codes applied during this study is that fluid flow in both fractures and matrix can be modeled via Darcy's equation, which reduces the fluid flow equations to a mass conservation equation. The governing equation for mass conservation equates the change in mass of fluid components over time with the component mass flux into the volume over the surface of the volume via diffusive and advective fluid transport, plus component mass source rates into the control volume:

$$
\frac{\partial}{\partial t} \int_{V_{n}}\left[\sum_{\gamma=l, g}\left(\phi \rho_{\gamma} s_{\gamma} \omega_{\gamma}^{i}\right)\right] d V_{n}=\int_{\Gamma_{n}}\left[\mathbf{F}^{i} \cdot \mathbf{n}\right] d \Gamma_{n}+\int_{V_{n}}\left[\sum_{\gamma=l, g}\left(\omega_{\gamma}^{i} m_{\gamma}\right)\right] d V_{n}
$$

where $\omega_{\gamma}^{i}$ is the mass fraction of component $i$ in fluid $\gamma$, and $\mathbf{F}^{i}$ is the combined advective and diffusive mass flux tensor of component $i(\mathrm{~kg} / \mathrm{s})$. Advective flux of components is via fluid flow and diffusive flux is via component diffusion within the fluid:

$$
\mathbf{F}^{i}=\sum_{\gamma=l, g}\left(\rho_{\gamma} \omega_{\gamma}^{i} \mathbf{V}_{\gamma}+\mathbf{J}_{\gamma}^{i}\right)
$$

where, $\mathbf{V}_{\gamma}$ is the Darcy velocity tensor of fluid $\gamma(\mathrm{m} / \mathrm{s})$ and $\mathbf{J}_{\gamma}^{i}$ is the diffusive flux tensor of component $i$ in fluid $\gamma(\mathrm{kg} / \mathrm{s})$. The diffusive flux depends on component concentration gradients, diffusion coefficients and fluid tortuosity factors:

$$
\mathbf{J}_{\gamma}^{i}=-\phi \rho_{\gamma} s_{\gamma} \frac{M^{i}}{M_{\gamma}}\left(\tau_{\gamma} D_{\gamma}^{i}\right) \nabla \chi_{\gamma}^{i}
$$

where, $M^{i}$ is the molecular weight of component $i(\mathrm{~kg} / \mathrm{kmol}), M_{\gamma}$ is the molecular weight of fluid $\gamma$ $(\mathrm{kg} / \mathrm{kmol}), \tau_{\gamma}$ is the tortuosity factor for fluid $\gamma, D_{\gamma}^{i}$ is the effective diffusion-dispersion coefficient $\left(\mathrm{m}^{2} / \mathrm{s}\right)$ of component $i$ in fluid $\gamma$, and $\chi_{\gamma}^{i}$ is the mole fraction of component $i$ in fluid $\gamma$.

\subsubsection{Fracture Transmissivity}

Fracture transmissivity is the product of permeability and fracture hydraulic aperture, following the cubic law (Jaeger et al. 2007): 


$$
T=k e=\frac{e^{3}}{12}
$$

where $T$ is the transmissivity $\left(\mathrm{m}^{3}\right), k$ is the permeability $\left(\mathrm{m}^{2}\right)$, and $e$ is the hydraulic aperture $(\mathrm{m})$.

\subsection{Rock Mechanics Modeling}

Numerical modeling of rock mechanics via the participating simulators was solved by one of two approaches; continuum or discrete fracture geomechanics. In the continuum approach the problem domain is spatially discretized (i.e., gridding or meshing). Solutions are sought for all node locations assuming elasticity with the incremental solution of plastic flow when either shear or tensile failure limits are reached. Within the continuum geomechanics approach, two discretization approaches were employed: 1) finite-element based with an implicit temporal formulation, or 2) finite-difference based with an explicit temporal formulation. The implicit approach requires the solution of a linear system whose order equaled the number of finite-element nodes times the number of unknowns per element. The explicit formulation does not require a global linear system solution, with the drawback being small time steps.

Two distinct approaches were used for modeling the mechanics of discrete fractures and fracture networks. In both approaches individual fractures are discretized and a stress field around the fracture elements yield shear and normal deformations of the fractures. Rock joint models are then used to transform those fracture deformations into changes in fracture apertures and permeability. In the solid finite element method the stresses on the fracture elements are determined from a finite element based solution of the continuum geomechanics equations ( $\mathrm{Fu}$ et al. 2013). In this approach the discrete fractures are mapped into the finite element mesh of the solid domain. In the discrete fracture approach the solid domain is not discretized and stresses on the fractures are commonly determined using the boundary element method, but other methods involve using finite elements to model the solid domain (Garipov et al. 2016).

The discrete fracture geomechanics is defined by integral equation or influence function, and only requires that the fractures or discontinuities be discretized. These approaches yield lower-order systems of algebraic equations, requiring only two-dimensional discretizations for three-dimensional problems and one-dimensional discretizations for two-dimensional problems. The discrete fracture approaches are founded on the original works of Crouch (Crouch 1976; Crouch and Starfield 1983) where the displacement discontinuity method is developed for homogeneous, isotropic, linearly elastic material for infinite, finite, and semi-infinite domains. The central concept of this approach is to create a number of displacement discontinuities, having normal and shear components, of unknown magnitude along the boundaries of a fracture, then set up and solve a system of algebraic equations to find the discontinuity values that produce the prescribed boundary tractions or displacements.

The discrete fracture and continuum geomechanics approaches are founded on the same equations of motion for bodies of rock mass; where the integral of the surface tractions acting on the boundary of the body plus the integral of the rock mass times body forces equal the acceleration of the rock mass (Jaeger et al. 2007):

$$
\nabla^{T} \tau+\rho \mathbf{F}=\rho \frac{\partial^{2} \mathbf{u}}{\partial t^{2}}
$$

where $\tau$ is the stress tensor $(\mathrm{Pa}), \rho$ is the rock mass density $\left(\mathrm{kg} / \mathrm{m}^{3}\right), \mathbf{F}$ is the body force per unit mass vector $(\mathrm{N} / \mathrm{m}), t$ is time (s), and $\mathbf{u}$ is the displacement vector $(\mathrm{m})$ : 


$$
\tau=\left|\begin{array}{ccc}
\tau_{x x} & \tau_{x y} & \tau_{x z} \\
\tau_{y x} & \tau_{y y} & \tau_{y z} \\
\tau_{z x} & \tau_{z y} & \tau_{z z}
\end{array}\right| ; \mathbf{F}=\left[\begin{array}{c}
F_{x} \\
F_{y} \\
F_{z}
\end{array}\right] ; \mathbf{u}=\left[\begin{array}{c}
u_{x} \\
u_{y} \\
u_{z}
\end{array}\right]
$$

For situations where rock displacements occur slowly or in the limit the rock is in static equilibrium, the equations of motion reduce to equations of equilibrium:

$$
\nabla^{T} \tau+\rho \mathbf{F}=0
$$

Equations (2.8) and (2.10) are independent of any stress-strain model, comprise three equations, one for each coordinate direction, but have at least six unknowns. Closure in these equations is provided by the six strain-displacement relationships and the stress-strain model:

$$
\varepsilon=\left[\begin{array}{ccc}
\varepsilon_{x x} & \varepsilon_{x y} & \varepsilon_{x z} \\
\varepsilon_{y x} & \varepsilon_{y y} & \varepsilon_{y z} \\
\varepsilon_{z x} & \varepsilon_{z y} & \varepsilon_{z z}
\end{array}\right]=\left[\begin{array}{ccc}
\frac{\partial u_{x}}{\partial x} & \frac{1}{2}\left(\frac{\partial u_{x}}{\partial y}+\frac{\partial u_{y}}{\partial x}\right) & \frac{1}{2}\left(\frac{\partial u_{x}}{\partial z}+\frac{\partial u_{z}}{\partial x}\right) \\
\frac{1}{2}\left(\frac{\partial u_{x}}{\partial y}+\frac{\partial u_{y}}{\partial x}\right) & \frac{\partial u_{y}}{\partial y} & \frac{1}{2}\left(\frac{\partial u_{y}}{\partial z}+\frac{\partial u_{z}}{\partial y}\right) \\
\frac{1}{2}\left(\frac{\partial u_{x}}{\partial z}+\frac{\partial u_{z}}{\partial x}\right) & \frac{1}{2}\left(\frac{\partial u_{y}}{\partial z}+\frac{\partial u_{z}}{\partial y}\right) & \frac{\partial u_{z}}{\partial z}
\end{array}\right]
$$

where, $\boldsymbol{\varepsilon}$ is the strain tensor. For example, the linear-elasticity stress-strain model relates the six components of the stress tensor with the six components of the strain tensor with the shear modulus and Lamé parameter:

$$
\begin{aligned}
& \tau_{x x}=(\lambda+2 G) \varepsilon_{x x}+\lambda \varepsilon_{y y}+\lambda \varepsilon_{z z} ; \quad \tau_{y y}=\lambda \varepsilon_{x x}+(\lambda+2 G) \varepsilon_{y y}+\lambda \varepsilon_{z z} \\
& \tau_{z z}=\lambda \varepsilon_{x x}+\lambda \varepsilon_{y y}+(\lambda+2 G) \varepsilon_{z z} ; \quad \tau_{x y}=\tau_{y x}=2 G \varepsilon_{x y}=2 G \varepsilon_{y x} \\
& \tau_{x z}=\tau_{z x}=2 G \varepsilon_{z y}=2 G \varepsilon_{z x} ; \quad \tau_{z y}=\tau_{y z}=2 G \varepsilon_{z y}=2 G \varepsilon_{y z}
\end{aligned}
$$

where, $\lambda$ is the Lamé parameter $(\mathrm{Pa})$ and $G$ is the shear modulus $(\mathrm{Pa})$. Linear elasticity is defined by two independent parameters, which can be related to a number of other parameters:

$$
\begin{aligned}
& \lambda=\frac{E v}{(1+v)(1-2 v)} ; \quad G=\frac{E}{2(1+v)} ; \quad K=\frac{E}{3(1-2 v)} ; \quad \lambda=\frac{2 G v}{(1-2 v)} \\
& E=2 G(1+v) ; \quad K=\frac{2 G(1+v)}{3(1-2 v)} ; \quad \lambda=K-\frac{2}{3} G ; \quad E=\frac{9 K G}{3 K+G} ; \quad v=\frac{3 K-2 G}{6 K+2 G}
\end{aligned}
$$

\subsubsection{Continuum Geomechanics}

Participating numerical simulators that approach geomechanical problems via the continuum approach use either implicit finite element methods (Deb 2006; Pande et al. 1990) or explicit finite difference methods (Itasca Consulting Group 2011, 2014). Finite element methods are numerical approaches in 
which the continua are discretized into small, finite elements. The geometry of these elements is relatively flexible allowing the modeling of complex physical systems. Node points are then distributed on the finite element depending on the degree of the polynomial that is chosen to mathematically describe the distribution of primary variables, such as displacement components, across the finite element. A set of simultaneous equations are then developed to solve either the equation of motion Eqn. (2.8), or the static equilibrium equation Eqn. (2.10) for each element in terms of the discrete nodal points. These equations are solved simultaneously, requiring a linear system solution, where the number of unknowns is equal to the total number of defined node points times the number of primary variables per node point. Finite element methods allow for heterogeneous distributions of geomechanical properties and can be used to identify rock mass failure, via a number of criteria models (e.g., Mohr-Coulomb (Mohr 1914), DruckerPrager (Drucker and Prager 1952), Hoek-Brown (Hoek and Brown 1980)). Finite element methods have additionally been applied to systems where the elasticity assumption is dropped and the elastic-plastic formulation of the stress-strain relationship is resolved via nonlinear solution methods. Moreover the finite element method has been applied to rock joints using discrete joint elements; where joint elements evolve dynamically with fracture propagation. Extended finite element methods (XFEM) (Moes et al. 1999; Mohammadi 2008; Pommier et al. 2011) are being developed to investigate crack propagation without the need for remeshing through the use of discontinuous enrichment terms. The mathematical background of the XFEM is the partition of unity concept. XFEM approaches are currently being developed for the GEOS numerical simulator, but were not fully implemented for the current study.

The mathematical foundation of the finite difference based method used in FLAC ${ }^{3 \mathrm{D}}$ is essentially identical to those used in the finite element based methods: a set of equations relating mechanical stress and kinematic strain rate are solved for particular geometries and properties for specified boundary and initial conditions (Itasca Consulting Group 2014). An important distinction between the two methods, however, is that FLAC ${ }^{3 \mathrm{D}}$ is formulated with the full inertial terms even for problems involving equilibrium or near equilibrium conditions. The FLAC ${ }^{3 \mathrm{D}}$ spatial discretization is based on tetrahedra, assuming linear distributions of the primary variables over the volumetric domain of the tetrahedron between the node points, located at the four apices of the tetrahedron. Temporal discretization also uses linear variations of the variables over time, but unlike the finite element method, the $\mathrm{FLAC}^{3 \mathrm{D}}$ approach solves the governing equations at the node points using an explicit time formulation, which eliminates the global linear system solution. The resulting equations are expressed in ordinary differential form with respect to time, and relate deformation rate with nodal velocities. The assumption of linear variation over time allows the differential equation in time to be evaluated using central finite differences, where nodal velocities are stored for times that are advanced by half time steps from the displacements and forces. The node locations and displacements are subsequently computed using the central difference approximation (Itasca Consulting Group 2014). The numerical formulation just described is termed the Large-Strain Mode in FLAC $^{3 \mathrm{D}}$. The simulator additionally provides a Small-Strain Mode, where small displacements, displacement gradients, and rotations are assumed, which translates numerically to static node coordinates (i.e., node translations and rotations are not taken into consideration).

\subsubsection{Discrete Fracture Geomechanics}

Whereas the continuum geomechanics approaches are considered with displacements of the bulk rock mass and any discontinuities, such as cracks, fractures and faults, the discrete fracture geomechanics approaches are specifically concerned with the normal and shear displacements of the fracture surfaces. Typical settings for EGS are formations with relatively low intrinsic permeability, where pre-existing or new fractures are stimulated to develop flow pathways. In the discrete fracture modeling approach, preexisting or potential new fractures are conceptualized as a network of discrete fractures, either in two or three dimensions. The governing equation for discrete fracture mechanics are founded on the assumption of quasi-static equilibrium in a rock continuum, assuming that the body forces are equal to zero: 


$$
\nabla^{T} \tau=0
$$

Fracture segments are considered to be either mechanically open or closed. Open fracture segments have walls that are not in contact and require the effective normal stress to be zero and the shear stress is zero:

$$
\sigma_{n}^{\prime}=\sigma_{n}-P=0, \tau=0
$$

where $\sigma_{n}^{\prime}$ is the effective normal stress $(\mathrm{Pa}), \sigma_{n}$ is the normal stress (Pa), $P$ is the fluid pressure (Pa), $\tau$ is the shear stress $(\mathrm{Pa})$. To approximate inertia in the shear stress component of open fracture segments, a radiation damping term can be included (Rice 1993):

$$
\sigma_{n}^{\prime}=\sigma_{n}-P=0, \tau-\left(\frac{G}{2 v_{s}}\right) v=0
$$

where $G$ is the shear modulus, $v_{s}$ is the shear-wave velocity $(\mathrm{m} / \mathrm{s})$ and $v$ is the shear velocity $(\mathrm{m} / \mathrm{s})$. Closed fracture segments have walls that are in contact with non-zero normal stress and nonzero shear stress:

$$
\sigma_{n}^{\prime}=\sigma_{n}-P>0,|\tau|=\mu_{f} \sigma_{n}^{\prime}+S_{o}
$$

where $\sigma_{n}^{\prime}$ is the effective normal stress $(\mathrm{Pa}), \sigma_{n}$ is the normal stress $(\mathrm{Pa}), P$ is the fluid pressure (Pa), $\tau$ is the shear stress $(\mathrm{Pa}), \mu_{f}$ is the coefficient of friction, and $S_{o}$ is the fracture cohesion $(\mathrm{Pa})$. As with open fracture segments inertia in the shear stress component can be approximated with a radiation damping term:

$$
\sigma_{n}^{\prime}=\sigma_{n}-P>0,\left|\tau-\left(\frac{G}{2 v_{s}}\right) v\right|=\mu_{f} \sigma_{n}^{\prime}+S_{o}
$$

The terms open and closed fractures have been used in the literature to mean those fractures that are hydraulically conducting and not, respectively. For this manuscript, mechanically closed fractures refer to those fractures with walls that are in contact, but contain and conduct fluids. Hydraulically closed fractures will refer to those fractures with walls that are in contact and do not conduct fluid.

\subsubsection{Displacement Discontinuity Method}

The governing equations for discrete fracture mechanics can be solved using the Displacement Discontinuity (DD) method, originally developed by Crouch and Starfield (Crouch and Starfield 1983). Shou and Crouch (Shou and Crouch 1995) further developed these approaches, where it was shown that quadratic basis functions increase the accuracy of stresses and displacements over a linear or constant basis function. The method of Shou and Crouch (Shou and Crouch 1995) calculates the stresses induced by shear and normal displacement discontinuities by satisfying the equations of quasi-static equilibrium and compatibility for small strain deformation in an infinite, two- dimensional, homogeneous, isotropic, linearly elastic medium in plane strain. The solution technique requires discretizing each discrete fracture into elements. The induced shear and normal stresses at each element $i$ caused by the shear and opening displacements of each element $j$ are linearly related through constant interaction coefficients: 


$$
\begin{aligned}
& \Delta \sigma_{i}=\left\{\sum_{j=1}^{n}\left(B_{E, \sigma}\right)_{i, j} \Delta E_{j}+\left(B_{D, \sigma}\right)_{i, j} \Delta D_{j}\right\} \\
& \Delta \tau_{i}=\left\{\sum_{j=1}^{n}\left(B_{E, \tau}\right)_{i, j} \Delta E_{j}+\left(B_{D, \tau}\right)_{i, j} \Delta D_{j}\right\}
\end{aligned}
$$

where $\Delta \sigma_{i}$ is the induced normal stress $(\mathrm{Pa})$ at element $i, \Delta \tau_{i}$ is the induced shear stress $(\mathrm{Pa})$ at element $i, \Delta E_{j}$ is the opening displacement (m) at element $j, \Delta D_{j}$ is the shear displacement (m) at element $j$, and $B_{E, \sigma}, B_{D, \sigma}, B_{E, \tau}, B_{D, \tau}$ are the constant matrices of interaction coefficients, calculated according to Shou and Crouch (Shou and Crouch 1995) . It is critical to note that induced normal and shear stresses at each element are dependent on the shear and opening displacements at all fracture elements. Shou and Crouch (Shou and Crouch 1995) additionally provide methods for handling fracture deviations and tips. The displacement discontinuity methods of Crouch and Starfield (Crouch and Starfield 1983) and Shou and Crouch (Shou and Crouch 1995) were used by the numerical simulators for this study, all of which involved two-dimensional calculations. Three-dimensional capabilities, which allow modeling twodimensional fractures and require discretization of the fracture surfaces into areas was recently completed for the CFRAC simulator, following the method of Okada (Okada 1992).

The Boundary Integral Equation Method (BEM) is a numerical technique for solving linear partial differential equations expressed in the form of integral equations. In the boundary element technique, a mesh or grid of elements is defined only on the surface of the body under consideration, and a system of simultaneous equations in terms of the unknowns associated with nodes of surface elements is solved. The boundary element method can be direct or indirect. The direct integral representation gives displacements at internal and boundary points in terms of boundary tractions and displacements. In the indirect integral representation, the displacements are written in function of variables which are not explicitly the boundary displacements or tractions (Brebbia and Dominguez 1977). In the context of solid mechanics analyses, the indirect boundary element method is comprised of two different techniques namely, the fictitious stress method and the displacement discontinuity method. The fictitious stress technique is applicable when the two surfaces of the boundary do not coincide in space (e.g., an underground opening). The displacement discontinuity is the most suitable method for fractures.

The boundary integral equations for the displacement discontinuity method can also be obtained formally from a direct BEM formulation using the principle of virtual work. It is, however, possible to heuristically construct the method using the principle of superposition which is less mathematical and permits a physical interpretation of the method. Its development began with attempts to model mining problems involving slit-like openings having one dimension much smaller compared with the other dimensions (Hackett 1959; Salamon 1963). Crouch (Crouch 1976) derived the fundamental solutions for a displacement discontinuity line segment in infinite and semi-infinite media. Crouch and Starfield (Crouch and Starfield 1983) further developed the technique by improvements and extensions involving inhomogeneous bodies and a three-dimensional formulation. Other investigators (Roegiers et al. 1982) use the point displacement discontinuity as their fundamental solution. This solution is then integrated over a desired element shape to form the building block of the DD method.

Higher order (quadratic) DD elements also have been extensively developed and used (Vandamme and Curran 1989). Shou and Crouch (Shou and Crouch 1995) proposed using a patch of constant elements to generate a quadratic DD variation using two degrees of freedom, improving the computational efficiency. Some of the codes presented here are based on poroelastic and thermos-poroelastic formulation. Details of the extension of the DD method to poroelasticity and thermo-poroelasticity can be found in Vandamme 
and Curran (Vandamme and Curran 1989), Vandamme and Roegiers (Vandamme and Roegiers 1990), and Ghassemi and Zhang (Ghassemi and Zhang 2006). The former also illustrate the difference between a fully coupled poroelastic DD formulation and a partial coupling used by some participants.

\subsubsection{Distinct Element Method}

The distinct element method (DEM) can be used to analyze coupled thermo-hydro-mechanical processes in discontinuous media (Itasca Consulting Group 2013). A discontinuous medium is distinguished from a continuous one by the existence of contacts or interfaces between the discrete bodies that make up the system. The distinct element method uses rigid or deformable blocks separated by contacts to represent a jointed rock mass. Distinct element programs use an explicit time-marching scheme to solve the equations of motion of the blocks directly.

The method consists of three parts which are conducted each time-step: (i) the block positions are used to calculate block contact forces, (ii) the forces and moments are used to calculate linear and angular accelerations of each block; block velocities and displacements are determined by integration over increments of time and (iii) a spatial search determines if any contacts have been created or removed. In the case of deformable blocks, additional degrees of freedom (grid points) are introduced into the blocks and the same algorithm is applied to the element grid points. Mechanical damping is used in the equations of motion to provide both dynamic and static solutions. Coupled joint fluid flow and thermal models are also available.

A rock joint is represented as a contact surface (composed of individual point contacts) formed between two block edges. Adjacent blocks can touch along a common edge segment, or at discrete points where a corner meets an edge or another corner. The distinct element method uses the soft-contact approach in which adjacent blocks are allowed to interpenetrate; the joint normal stress is a function of the interpenetration length. Many joint constitutive models for block contacts can be used with the distinct element method. The Coulomb slip joint model is found to capture many of the features that are representative of the physical response of rock joints, this model is described here. An increment in joint normal displacement $\Delta u_{n}$ results in a linear increase in joint normal stress $\Delta \sigma_{n}$ described by,

$$
\Delta \sigma_{n}=-k_{n} \Delta u_{n}
$$

where $k_{n}$ is the joint normal stiffness. A limiting tensile strength $T$ exists for each joint such that $\sigma_{n}=0$ if $\sigma_{n}<-T$. The joint shear stress, $\tau_{s}$, is limited by a combination of a cohesive, $C$, and

frictional, $\phi$, strength. The maximum joint shear stress, $\tau_{\max }$, is given by,

$$
\tau_{\max }=C+\sigma_{n} \tan \phi
$$

When $\left|\tau_{s}\right| \geq \tau_{\text {max }}$, then $\tau_{s}=\operatorname{sign}\left(\Delta u_{s}\right) \tau_{\max }$. In the absence of frictional sliding an increment of joint shear displacement, $\Delta u_{S}$, results in a linear increase in joint shear stress described by,

$$
\Delta \tau_{s}=-k_{s} \Delta u_{s}
$$

where $k_{s}$ is the joint stiffness. 
Joint dilation may occur during non-elastic joint sliding, this may be an important mechanism for increasing the permeability of enhanced geothermal reservoirs by high pressure water injection. Dilation is described by the dilation angle $\psi$. An increment of dilatational normal displacement results from a shear displacement according to,

$$
\Delta u_{n}^{d}=\Delta u_{s} \tan \psi
$$

The dilation angle, $\psi$, is zero when frictional slip is not occurring or if the accumulated shear displacement exceeds a predefined limit. If the shear displacement increment is in the same direction as the total shear displacement, dilation increases or decreases otherwise. Displacement weakening and Barton-Bandis joint models are also available.

Distinct blocks can either be rigid or deformable. Deformable blocks are directly represented by discretizing the blocks into triangular plane strain elements. The force in a grid point is

$$
F_{i}^{T}=\int_{s} \sigma_{i, i} n_{j} d s+F_{i}^{c}+F_{i}^{l}
$$

where $\sigma_{i, i}$ is the stress tensor in the zone adjacent to the grid point, $n_{j}$ is the normal of the surface $s$ which delimits the grid point mass, $F_{i}^{c}$ and $F_{i}^{l}$ are contact forces and external loads. Dots denote derivatives with respect to time and repeated indices imply summation. Strain is related to grid point displacement via

$$
\dot{\varepsilon}=0.5\left(\dot{u}_{i, j}-\dot{u}_{j, i}\right)
$$

where the commas in the subscripts denote derivatives.

A block constitutive model is an arbitrary function which takes a strain increment as input and returns a stress increment. The new stress results in new grid point forces. Non-linear and post peak constitutive models are readily incorporated into this framework. Isotropic linear elastic constitutive behavior can be written as

$$
\Delta \sigma_{i, j}^{e}=\lambda \Delta \varepsilon_{v} \delta_{i, j}+2 \mu \Delta \varepsilon_{i, j}
$$

where, $\lambda$ and $\mu$ are the Lame constants, $\Delta \sigma_{i, j}^{e}$ are the elastic increments of the stress tensor, $\Delta \varepsilon_{i, j}$ are the incremental strains, $\Delta \varepsilon_{v}$ is the increment of volumetric strain and $\delta_{i, j}$ is the Kronecker delta function.

The joint model and block deformation model described above are used to determine the force on each grid point (or block centroid in the case of rigid blocks). The equation of motion of a grid point (or rigid block) is

$$
\frac{d \dot{u}_{i}}{d t}=\frac{F_{i}^{T}+F_{i}^{d}}{m}+g_{i}
$$


where, $F_{i}^{T}$ is the total unbalanced grid point force (consisting of contact forces, external loads and stresses from adjacent zones), $F_{i}^{d}$ is a mechanical damping force described below, $m$ is the grid-point mass and $g_{i}$ is the acceleration due to gravity. A finite difference approximation of the transient term is made

$$
\frac{d \dot{u}_{i}}{d t}=\frac{\dot{u}_{i}^{t+\Delta t / 2}-\dot{u}_{i}^{t-\Delta t / 2}}{\Delta t}
$$

where, $\Delta t$ is the integration time step. Rearranging, substituting, and defining velocities at half time steps gives,

$$
\dot{u}_{i}^{t+\Delta t / 2}=\dot{u}_{i}^{t-\Delta t / 2}+\left(\frac{F_{i}^{T}+F_{i}^{d}}{m}+g_{i}\right) \Delta t
$$

The grid-point (or rigid-block) locations, $x_{i}$ are updated as,

$$
x_{i}^{t+\Delta t}=x_{i}^{t}+\dot{u}_{i}^{t+\Delta t / 2} \Delta t
$$

The new grid point locations yield new contact forces and zone stresses and the calculation continues.

For static (non-inertial) problems a form of mechanical damping referred to as local damping is used to dissipate kinetic energy and quickly approach the steady state. Local damping is proportional to the unbalanced force magnitude and is applied in the direction opposite the unbalanced force. The force is defined as

$$
F_{i}^{d}=-\alpha\left|F_{i}^{T}\right| \operatorname{sign}\left(\dot{u}_{i}^{t-\Delta t / 2}\right)
$$

where, $\alpha$ is a constant typically taken as 0.8 . This force is added to the total grid point force during the time integration.

An efficient spatial search algorithm is used to find new contacts and remove old contacts as blocks are displaced. For the purpose of contact resolution only block corners are represented as a circular arc whose tangent point is a constant distance from the block apex. This approximation is taken to avoid numerical artifacts (corner locking), typical rounding lengths are on the order of $1 \%$ of the characteristic block length.

\subsubsection{Joint Models}

Fractures are a nearly ubiquitous feature of rock masses and help distinguish geomechanics from the mechanics of other engineering materials. For this manuscript, closed fractures are considered to have surface contact in some regions, but separated in others. Fractures that have undergone significant shear will be classified as faults and those that have not will be denoted as joints (Mandl 2000). Both the continuum and discrete fracture modeling approaches use relationships to relate effective normal stress and cumulative shear displacement to void (volume of fluid stored per fracture surface area) and hydraulic (fluid transmittance) aperture. A number of relationships were used by the participating numerical simulators. 
There is not a particular set of equations that are universally accepted for modeling joint closure. One common method is to define the void and hydraulic aperture of a closed fracture as a function of the effective normal stress and cumulative shear displacement (Kohl and Megel 2007; Rahman et al. 2002; Willis-Richards et al. 1996):

$$
\begin{gathered}
E=\frac{E_{o}}{1+9\left(\frac{\sigma_{n}^{\prime}}{{ }^{*} \sigma_{n}^{E}}\right)}+D_{E, \text { eff }} \tan \left(\frac{\varphi_{\text {dil }}^{E}}{1+9\left(\frac{\sigma_{n}^{\prime}}{{ }^{*} \sigma_{n}^{E}}\right)}\right) ; \quad D_{\text {eff }}^{E}=\min \left[D,{ }^{*} D_{\text {eff }}^{E}\right] \\
e=\frac{e_{o}}{1+9\left(\frac{\sigma_{n}^{\prime}}{{ }^{*} \sigma_{n}^{e}}\right)}+D_{e, \text { eff }} \tan \left(\frac{\varphi_{\text {dil }}^{e}}{1+9\left(\frac{\sigma_{n}^{\prime}}{{ }^{*} \sigma_{n}^{e}}\right)}\right) ; \quad D_{\text {eff }}^{e}=\min \left[D,{ }^{*} D_{\text {eff }}^{e}\right]
\end{gathered}
$$

where, $E$ or $e$ is the void or hydraulic aperture $(\mathrm{m}), E_{o}$ is the reference void or hydraulic aperture $(\mathrm{m})$, $\sigma_{n}^{\prime}$ is the effective normal stress $(\mathrm{Pa}),{ }^{*} \sigma_{n}^{E}$ or ${ }^{*} \sigma_{n}^{e}$ is the void- or hydraulic-aperture reference fracture stiffness (Pa), $\varphi_{\text {dil }}^{E}$ or $\varphi_{\text {dil }}^{e}$ is the void- or hydraulic aperture dilation angle ( $\left.{ }^{\circ}\right),{ }^{*} D_{\text {eff }}^{E}$ or ${ }^{*} D_{e f f}^{e}$ is the maximum void- or hydraulic- aperture cumulative sliding displacement (m), and $D_{e f f}^{E}$ or $D_{e f f}^{e}$ is the voidor hydraulic- aperture cumulative sliding displacement $(\mathrm{m})$. A non-zero aperture dilation angle corresponds with aperture dilation with slip along the fracture. The void and hydraulic aperture of an open fracture is defined as a function of the cumulative shear displacement and the physical separation of the fracture surfaces (McClure and Horne 2013):

$$
\begin{aligned}
& E=E_{o}+D_{\text {eff }}^{E} \tan \left(\varphi_{\text {dil }}^{E}\right)+E_{\text {open }} \\
& e=e_{o}+D_{\text {eff }}^{e} \tan \left(\varphi_{\text {dil }}^{e}\right)+e_{\text {open }}
\end{aligned}
$$

Similar equations can be written for the void aperture of a closed fracture, following the Barton-Bandis formulations (Barton 1976; Barton 2013; Barton et al. 1985):

$$
E=E_{o}-\frac{\sigma_{n}^{\prime}}{\frac{k_{n}}{A}+\frac{\sigma_{n}^{\prime}}{d_{\max }}}+\Delta \delta \tan \left(d_{n}^{\circ}\right)
$$

where, $E$ is the void aperture (m), $E_{o}$ is the reference or initial void aperture (m), $\sigma_{n}^{\prime}$ is the effective normal stress $(\mathrm{Pa}), k_{n}$ is the normal stiffness of the fracture $(\mathrm{N} / \mathrm{m}), A$ is the surface area of the fracture $\left(\mathrm{m}^{2}\right), d_{\max }$ is the maximum fracture closure $(\mathrm{m}), \Delta \delta$ is the shear displacement $(\mathrm{m})$, and $d_{n}^{\circ}$ is the joint dilation angle. The reference or initial void aperture can be related to three joint characterization parameters (Barton et al. 1985): 


$$
E_{o}=\frac{J R C}{5}\left(0.2 \frac{\sigma_{c}}{J C S}-0.1\right)
$$

where, JRC is the joint roughness coefficient, JCS is the joint wall compressive strength $(\mathrm{Pa})$, and $\sigma_{C}$ is the unconfined compressive strength of the rock. Furthermore the joint roughness coefficient typically varies between zero to 15 and can be determined from experimental tests:

$$
J R C=\frac{\alpha-\phi_{r}}{\log \left(\frac{J C S}{\sigma_{n o}^{\prime}}\right)}
$$

where $\alpha$ is the tilt angle when sliding occurs, $\phi_{r}$ is residual friction angle, and $\sigma_{n o}^{\prime}$ is the effective normal stress $(\mathrm{Pa})$ when sliding occurs. The joint characterization parameters can additionally be used to determine the joint dilation angle (Barton et al. 1985):

$$
d_{n}^{\circ}=\frac{1}{2} J R C \log \left(\frac{J C S}{\sigma_{n}^{\prime}}\right)
$$

Conversion between void aperture and hydraulic aperture for the Barton-Bandis model makes use of the joint roughness coefficient:

$$
e=\frac{E^{2}}{J R C^{2.5}}
$$

An alternative approach to relating the mean effective normal stress to the fracture aperture using the Barton-Bandis model involves two material constants defined via three parameters. In this model mean effective normal stress is defined as a function of the mechanical aperture (Fu et al. 2013):

$$
\sigma_{n}^{\prime}=\frac{E^{\max }-E}{A-B\left(E^{\max }-E\right)}
$$

where $A(\mathrm{~m})$ and $B$ are two material constants. These constants can be determined from a reference normal effective stress and mechanical aperture:

$$
A=E^{\max }\left(\frac{E^{\max }-{ }^{*} E}{{ }^{*} \sigma_{n}^{E}{ }^{*} E}\right) ; \quad B=\left(\frac{E^{\max }-{ }^{*} E}{{ }^{*} \sigma_{n}^{E}{ }^{*} E}\right)
$$

where ${ }^{*} \sigma_{n}^{E}$ is the reference normal effective stress $(\mathrm{Pa})$, and ${ }^{*} E$ is the reference mechanical aperture.

\subsection{Geochemical Reaction Modeling}

Modern numerical simulators with capabilities for modeling geochemical reactions generally consider a variety of equilibrium and kinetic process types: 1) mineral dissolution/precipitation, 2) homogeneous aqueous, 3) surface complexation, 4) ion exchange, 5) fluid exchange (e.g., aqueous-gas, aqueousnonaqueous liquid), and 6) microbially mediated. For EGS the principal reaction processes of concern are mineral dissolution/precipitation, which are generally considered to be kinetic in nature; and aqueous based reactions, normally sufficiently rapid to be considered to be equilibrium in nature. The geochemical 
chemical system is defined by the number of independent chemical species and the reactions between the species. For mathematical closure an equation is needed for every chemical species; where, the equations can express chemical equilibrium, kinetic reactions, or conservation of total concentration.

Equilibrium equations, often referred to as mass action equations, relate species activities through an equilibrium constant (White and McGrail 2005):

$\left(\gamma_{j} C_{j}\right)=K_{j} \prod_{i=1}^{N_{j}^{e q}}\left(\gamma_{i} C_{i}\right)^{v_{i, j}}$

where, $\gamma_{j}$ is the activity coefficient of species $j, C_{j}$ is the concentration of species $j\left(\mathrm{kmol} / \mathrm{m}^{3}\right), K_{j}$ is the equilibrium constant for the equilibrium reaction $j, N_{j}^{e q}$ is the number of species in the equilibrium reaction $j$, and $v_{i, j}$ is the stoichiometric coefficient for species $i$ in the equilibrium reaction $j$. Equilibrium constants are frequently expressed as having temperature dependence.

Kinetic equations equate the time rate of change of a collection of species with a reaction rate (White and McGrail 2005):

$$
\frac{d \sum_{i=1}^{N_{s p}^{k n}}\left(b_{i} C_{i}\right)}{d t}=\sum_{k=1}^{N_{r t}^{k n}}\left(c_{k} R_{k}\right)
$$

where, $N_{s p}^{k n}$ is the number of species in the kinetic equation, $b_{i}$ is the kinetic-equation stoichiometric coefficient of species $i, C_{i}$ is the concentration of species $i\left(\mathrm{kmol} / \mathrm{m}^{3}\right), N_{r t}^{k n}$ is the number of kinetic reaction rate mechanisms, $c_{k}$ is the weighting parameter for reaction rate $k$, and $R_{k}$ is the rate for reaction rate $k(\mathrm{kmol} / \mathrm{s})$.

Conservation equations are used to close the system of geochemical equations. The number of conservation equations needed to close the system equals the total number species minus the number of equilibrium equations, Eqn. (2.20), minus the number of kinetic equations, Eqn. (2.21). Conservation equations define total concentrations or basis species and specify that the time rate of change of the total concentration of a basis species is zero (White and McGrail 2005):

$$
\frac{d \sum_{i=1}^{N_{s p}^{c n}}\left(v_{i, j} C_{i}\right)}{d t}=\frac{d \Psi_{j}}{d t}=0 ; \quad \text { for } i=j, v_{i, j}=1
$$

where, $N_{s p}^{c n}$ is the number of species that make up the total concentration, and $\Psi_{j}$ is the total concentration of species $j$. 


\subsubsection{Aqueous Reaction Rates}

Aqueous reaction rates generally appear in either a reversible or irreversible form (Steefel et al. 2014). The reversible form can be written with a transition state theory (TST) type rate law:

$$
R_{k}=k_{k}\left(1-\frac{Q_{k}}{K_{k}}\right) \prod\left(\gamma_{i} C_{i}\right)^{n}
$$

where, $R_{k}$ is the rate for reaction rate $k(\mathrm{kmol} / \mathrm{s}), k_{k}$ is the rate constant for reaction rate $k\left(\mathrm{~m}^{3} / \mathrm{s}\right), Q_{k}$ is the ion activity product for reaction rate $k, K_{k}$ is the equilibrium constant for reaction rate $k$. The ion activity product depends on the activities of the species in the kinetic equation:

$$
Q_{k}=\frac{\prod_{j=1}^{N_{j}^{e q}}\left(\gamma_{j} C_{j}\right)^{v_{i, j}}}{\left(\gamma_{i} C_{i}\right)}
$$

The term $\left(1-\frac{Q_{k}}{K_{k}}\right)$ drives the reaction toward equilibrium, with the sign of the reaction rate changing as the ion activity product transitions across the equilibrium point. The term $\prod\left(\gamma_{i} C_{i}\right)^{n}$ is the product of the far from equilibrium effects on the reaction rate (e.g., $\mathrm{pH}$ ), which promote or inhibit the reaction, with the product being over all species and the exponent being zero for species that have no effect on the reaction rate. The irreversible form of the aqueous reaction rate equation eliminates the thermodynamic equilibrium term:

$$
R_{k}=k_{k} \prod\left(\gamma_{i} C_{i}\right)^{n}
$$

\subsubsection{Mineral Precipitation/Dissolution Rates}

Mineral precipitation/dissolution reactions may be considered sufficiently rapid to be modeled as being in equilibrium, but in general are considered to be slower than aqueous reaction rates and are modeled as being kinetic. A number of reaction models have been developed that are distinguished by processes and controls. The transition state theory reaction rate models consider precipitation and dissolution to be reversible processes, with the dependence on the species saturation state being implicitly included (Steefel et al. 2014):

$$
R_{m}=\operatorname{sgn}|\Omega| A_{m} k_{m} \prod\left(\gamma_{i} C_{i}\right)^{n}\left|\left(\frac{Q_{m}}{K_{e q}}\right)^{\eta}-1\right|^{\lambda} ; \operatorname{sgn}|\Omega|=\operatorname{sgn}\left|\log \left(\frac{Q_{m}}{K_{e q}}\right)\right|
$$

where $\operatorname{sgn}|\Omega|$ gives the sign of the reaction (i.e., negative for dissolution, positive for precipitation), $A_{m}$ is the exposed specific surface area of the mineral $\left(\mathrm{m}^{2} / \mathrm{m}^{3}\right), k_{m}$ is the reaction rate $\left(\mathrm{kmol} / \mathrm{s} \mathrm{m}^{2}\right)$,

$\prod\left(\gamma_{i} C_{i}\right)^{n}$ is the activity product of the far from equilibrium effects on the reaction (e.g., $\left.\mathrm{pH}\right), Q_{m}$ is 
the aqueous ion activity product, and $K_{e q}$ is the equilibrium constant. Both the reaction rate and equilibrium constant are generally considered to be temperature dependent. The exponents $\eta$ and $\lambda$ are generally determined experimentally. A common temperature dependence model for the reaction rate makes uses of a reference temperature (e.g., 293.15 K) and rate (White and McGrail 2005):

$$
k_{m}={ }^{*} k_{m}\left[\frac{-E_{a}}{R}\left(\frac{1}{T}-\frac{1}{{ }^{*} T}\right)\right]
$$

where, ${ }^{*} k_{m}$ is the reference reaction rate $\left(\mathrm{kmol} / \mathrm{s} \mathrm{m}^{2}\right), E_{a}$ is the activation energy $(\mathrm{J} / \mathrm{kmol}), R$ is the ideal gas constant $(\mathrm{J} / \mathrm{kmol} \mathrm{K}), T$ is the temperature $(\mathrm{K})$, and ${ }^{*} T$ is the reference temperature $(\mathrm{K})$.

Dissolution- or precipitation-only mineral rate models are used to describe reactions that are not considered to be reversible or an extremely slow reversible reaction, therefore they do not include the equilibrium driving term. As these types of reaction rate models can have discontinuities across the equilibrium point, they can yield numerical difficulties. Irreversible mineral reaction rate laws have no dependence on equilibrium conditions, nor on the activities of the mineral species, and reactions may proceed well beyond the thermodynamic equilibrium point, but can have a dependence on the concentration of a remote species (e.g., $\mathrm{pH}$ ):

$$
R_{m}=A_{m} k_{m} \prod\left(\gamma_{i} C_{i}\right)^{n}
$$





\subsection{Numerical Solution Schemes}

Numerically simulating coupled thermal, hydrologic, geomechanical and geochemical (THMC) processes have been approached in a vast number of ways, and a complete review of the possible formulations is beyond the scope of this paper. Moreover, most of the numerical simulators for EGS involve the solution of large linear or nonlinear systems of equations and, linearization of the nonlinear systems commonly relies on Newton-Raphson techniques. These methods and their application in EGS simulators are well documented in the literature and will not be described in this paper. We will present specific and general numerical schemes for solving coupled THMC processes in subsurface environments. The global implicit solution, where all of the governing equations are solved simultaneously is possible, but is generally considered to be impractical for anything but small to moderate problems. The art of numerical simulation is clearly displayed in the ingenuity of code developers in creating methods for modeling complex THMC coupled processes.

\subsection{Sequential Schemes}

A straightforward method for avoiding a global implicit solution is to split the coupled THMC processes into unit process operations that are solved sequentially. The unit process operations are then coupled together via key input and output parameters (e.g., temperature, pressure, stress, aperture, porosity, permeability). If changes in the key parameters are small between unit process operation computations, then sequential schemes can yield accurate results. Time step size is generally the controlling factor for limiting changes in key input and output parameters between the unit process operation executions. A common sequential scheme is one where the governing equations for heat transport and multifluid flow are solved fully coupled, followed by geomechanics, and then geochemistry. Within the geochemistry unit operation it is additionally typical to use operator splitting, where a single time step is split into transport and reaction operations. Transport involves the migration of total species concentrations between grid cells based on the solution of the heat transport and multifluid flow field, and then geochemistry occurs within a grid cell, modifying the chemical composition of the grid cell. The reaction operation is analogous to conducting the reactions in individual Parr vessels at temperature and pressure. For this scheme, the key parameters output from the coupled heat transport and multifluid flow process computation are the temperature, pressure, fluid saturation, and fluid flow field. Key parameter inputs to the geomechanics computation are temperature and pressure, with key parameter outputs generally being stress, deformations, and changes in porosity, and permeability. Key parameter inputs to the geochemistry computation are temperature, pressure, fluid saturation, and the fluid flow field, with key parameter outputs being volumes of mineral precipitation/dissolution, and changes in porosity, and permeability.

\subsection{Iterative Schemes}

Iterative schemes also divide the coupled THMC processes into sequential unit operations, but then repeat the operations until convergence is reached on the key input and output parameters. Iterative schemes are specifically designed to overcome the small-time-step requirement of pure sequential schemes. Iteration between unit process operations can be limited to a subset of operations, with common subsets being THM and THC.

\subsubsection{Split Solid and Fracture Mechanics}

One simulation strategy specifically developed to model long-term production of an EGS reservoir is one that combines continuum heat transport and multifluid flow with separate solid and fracture mechanics 
(Fu et al. 2013). This scheme comprises four process computations: 1) discrete fracture network (Discrete-Fracture), 2) solid geomechanics via finite elements (Solid-FEM), 3) rock joint model (RockJoint), and 4) a continuum-based heat transport and fluid flow (Continuum-TH). The scheme is initiated with an iterative sequence between the Discrete-Fracture and Rock-Joint models, with the effective stress on the fracture and aperture width being the key input and output parameters, until the solution converges. The discrete fracture network is then mapped onto a finite difference mesh to create an equivalent permeability field for the Continuum-TH computations. The key output parameter from the ContinuumTH computations is temperature, which is used as input for the Solid-FEM computations. The key output parameter from the Solid-FEM computation is then the total stress, which serves to update the total stress on each fracture element of the discrete fracture network. Each new time step is initiated with the updated total stress field, which is used as key input parameters for the Discrete-Fracture computations and iteration with the Rock-Joint models.

\subsubsection{Implicit Sequential Solutions}

Sequential solutions of THMC unit process computations often involve executing independent computer codes, which could potentially execute on different spatial discretizations. For these situations, coupling modules are required to translate the key output and input parameters between the unit process computations (Rutqvist et al. 2002). An iterative solution strategy for modeling coupled THM processes that involves independent computer codes, using different spatial discretizations has been developed that provides two options for sequential TH and M process computations. This scheme comprises four elements: 1) a continuum-based heat transport and fluid flow (Continuum-TH) process computation, 2) a thermal-hydrologic to geomechanical coupling module (TH-M-Coupling), 3) a continuum-based geomechanics (Continuum-M) process computation, and 4) a geomechanical to thermal-hydrologic coupling module (M-TH-Coupling). The scheme is initiated with the Continuum-TH computation, which generates temperature, pressure, and fluid saturations. The TH-M-Coupling module then converts these into temperature and pressure inputs for the Continuum-M process computation. Key outputs from the Continuum-M computations are the effective stress tensor, which are then converted to changes in porosity, permeability, and entry pressure through the M-TH-Coupling module.

The multiple code and grid sequential scheme described above can be implemented in an explicit sequential manner, yielding a single unit process calculation each time step. The Continuum-TH calculation is carried out over the time step using old values of porosity, permeability and entry pressure, yielding changes in temperature and pressure, which are then used for the Continuum-M calculation to generate changes in stress and strain. The updated stress and strain tensors are then used to update the porosity, permeability, and entry pressure to initialize the next time step. The Continuum-TH calculation is nonlinear and requires an iterative solution scheme to reach convergence. In the implicit sequential procedure (Rutqvist et al. 2002) the Continuum-M calculations and associated updates to porosity, permeability, and entry pressure are executed after each iteration of the Continuum-TH calculation, not after the completed time step. With this scheme the resulting porosity, permeability and entry pressures at the close of the time step satisfy both the Continuum-TH and Continuum-M process calculations.

\subsection{Explicit Schemes}

A common thread across the numerical simulators used in this study is the discretization of time and space. Spatial discretization involves dividing solid domains into discrete volumetric or areal elements or dividing fractures into discrete areal or linear elements, depending on the problem dimensionality. Temporal discretization involves dividing time into discrete steps. Implicit solution schemes seek the solution of the governing equations at a future point in time for all discrete spatial points, requiring a global solution of the governing equations, with any nonlinearities being resolved through iterative 
schemes. In contrast, explicit solution schemes seek the solution of the governing equations at a future point in time for individual discrete spatial points; where contributions from other spatial points are based on values at the previous time step. From a computational perspective implicit schemes, with their requirement for a global solution, are more expensive per time step than explicit schemes, but the explicit schemes require smaller time steps to remain stable.

The FLAC ${ }^{3 D}$ (Itasca Consulting Group 2014) geomechanical simulator, used by a number of participating teams in this study, has an explicit finite difference formulation in time. Spatial discretization in FLAC ${ }^{3 \mathrm{D}}$ divides the three-dimensional domain into discrete tetrahedra, with the solutions being sought at node points located at the four vertices of the tetrahedron. In this explicit scheme, the velocity of a node point is assumed to vary linearly with time over the time step interval, and velocities are computed at half-timesteps with respect to displacements and forces (Itasca Consulting Group 2014):

$$
v_{i}^{\langle l\rangle}\left(t+\frac{\Delta t}{2}\right)=v_{i}^{\langle l\rangle}\left(t-\frac{\Delta t}{2}\right)+\frac{\Delta t}{M^{\langle l\rangle}} F_{i}^{\langle l\rangle}\left(t,\left\{v_{i}^{\langle 1\rangle}, v_{i}^{\langle 2\rangle}, v_{i}^{\langle 3\rangle}, \ldots, v_{i}^{\langle p\rangle}\right\}^{\langle l\rangle}, \kappa\right)
$$

where, $v_{i}^{\langle l\rangle}$ is the $i$ component of the velocity vector at global node point $\langle l\rangle(\mathrm{m} / \mathrm{s}), t$ is time (s), $\Delta t$ is the time step (s), $M^{\langle l\rangle}$ is the nodal mass (kg), and $F_{i}^{\langle l\rangle}$ is the out-of-balance force (N), which is a function values from all the tetrahedron that have vertices at global node point $\langle l\rangle$. Node locations and nodal displacements are updated using central differencing (Itasca Consulting Group 2014):

$$
\begin{aligned}
& x_{i}^{\langle l\rangle}(t+\Delta t)=x_{i}^{\langle l\rangle}(t)+\Delta t v_{i}^{\langle l\rangle}\left(t+\frac{\Delta t}{2}\right) \\
& u_{i}^{\langle l\rangle}(t+\Delta t)=u_{i}^{\langle l\rangle}(t)+\Delta t v_{i}^{\langle l\rangle}\left(t+\frac{\Delta t}{2}\right) ; u_{i}^{\langle l\rangle}(0)=0
\end{aligned}
$$

where, $x_{i}^{\langle l\rangle}(t)$ is the $i$ component of the node location at global node point $\langle l\rangle(\mathrm{m})$ at time $t$, and $u_{i}^{\langle l\rangle}(t)$ is the $i$ component of the nodal displacement at global node point $\langle l\rangle(\mathrm{m})$ at time $t$.

The time stepping criterion for this explicit scheme is based on conceptualizing the mechanical system as a collection of nodal masses connected with springs, whose spring constants are dependent on the bulk and shear modulus of the rock. For simulating oscillating mass-spring systems with an explicit finite difference scheme, time step increments are restricted to values below the minimum natural periods of the total system (Itasca Consulting Group 2014). Natural periods of oscillating mass spring systems can be determined from a global eigenvalue analysis, but this would be impractical for a practical spatial discretization of an EGS system. For an infinite series of uniform masses and springs, the time step limit can be expressed as a function of the mass and spring constant:

$$
\Delta t \leq \sqrt{\frac{m}{k}}
$$

where, $m$ is the mass $(\mathrm{kg})$ and $k$ is the spring constant $(\mathrm{N} / \mathrm{m})$. This infinite series criterion can be extended to a single tetrahedron by interpreting the mass as the nodal mass contribution at a local node, and the spring constant as the corresponding nodal stiffness contribution. The resulting time step limit is 
the minimum time step determined from a local analysis at global node point $\langle l\rangle$ (Itasca Consulting Group 2014):

$$
\Delta t \leq \sqrt{\frac{m^{\langle l\rangle}}{\left[\frac{\left(K+\frac{4}{3} G\right)}{9 V} \max \left(\left[n_{i}^{\langle l\rangle} S_{i}^{\langle l\rangle}\right]^{2}, i=1,3\right)\right]}}
$$

where, $m^{\langle l\rangle}$ is the tetrahedron mass contribution at a global node point $\langle l\rangle(\mathrm{kg}), K$ is the bulk modulus (Pa), $G$ is the shear modulus (Pa), $V$ is the volume of the tetrahedron $\left(\mathrm{m}^{3}\right)$, and $n_{i}\langle l\rangle S_{i}\langle l\rangle$ is the product of the unit surface normal vector and surface area $\left(\mathrm{m}^{2}\right)$.

\subsection{Functionalization Schemes}

Numerical simulation often requires repeated simulations of a particular physical process (e.g., heat transport, fluid flow, geomechanics, and geochemistry) conducted on a fixed model domain with varying driving forces (i.e., boundary conditions, sources, and sinks). The direct solution approach to this situation is to conduct the simulations with a mechanistic numerical simulator for the particular process or coupled processes. An alternative approach is to conduct a series of numerical simulations with a mechanistic model with different driving forces to develop a functional representation of the system The functional representation, with its lower execution time requirements, is then used to model the physical processes subject to different driving forces. Formulation of the functional representation requires a number of executions of the mechanistic process simulator, and a number of options are available for developing the representation: intuitive linear with a step-wise boundary function, analytical approach, quasi-linear, nonlinear using Volterra series solution, and nonlinear matrix model using Volterra series solution (Danko 2006). The formulation can be extended to multiple variables and multiple dimensions.

For EGS systems the functionalization approach has been applied in a unique fashion to solve coupled THMC problems with discrete fractures (Danko and Bahrami 2012; Danko et al. 2012). In this approach three independent numerical simulators are executed to develop functional representations for a particular physical domain with a discrete fracture network for three processes: 1) coupled heat transport and fluid flow in geologic media, 2) geomechanics and 3) geochemistry. Heat transport and fluid flow within the discrete fracture network are then modeled via a solution of the Navier-Stokes equations. The overall scheme melts these components together using iterative loops with successive approximation between the functional representations and the discrete fracture model equations. The scheme starts by computing bulk fluid flow in the discrete fracture network, assuming initial distributions of temperature, pressure, fracture aperture, and species concentrations. These results are then used to iterate between the discrete fracture network and the three functional representations for coupled heat transport and fluid flow, geomechanics and geochemistry between the continuum domain and discrete fractures. 


\subsection{Code Descriptions}

\subsection{AD-GPRS}

Automatic-Differentiation General Purpose Research Simulator (AD-GPRS) (Younis 2011; Zhou 2012) is a reservoir modeling platform developed and sustained by the SUPRI-B research group in the Energy Resources Engineering Department at Stanford University. AD-GPRS allows the flexible treatment of all nonlinear physics or formulations through the automatic differentiation framework inherent in the simulator (Voskov and Tchelepi 2012; Voskov et al.). Other AD-GPRS options include flexible MultiPoint Flux Approximation discretization and general Adaptive Implicit Method (Zhou et al.) OpenMP and Multi-GPU parallel implementation (Tchelepi and Zhou; Zhou 2012), advanced thermalcompositional formulation (Iranshahr et al. 2010; Zaydullin et al.) fully-coupled geomechanics for fractured media (Garipov et al. 2012) and adjoint-based optimization (Kourounis et al. 2010; Voskov and Volkov).

The main focus of AD-GPRS is to provide a flexible reservoir simulation research simulator that focuses on an extendable structure. Consequently, a modular object-orientated code structure was implemented. AD-GPRS focuses on providing a flexible, efficient and extendable numerical framework that is designed for reservoir simulation research. AD-GPRS solves the governing mass and energy balance equations with the finite volume method and linearizing the nonlinear equations using Newton-Raphson method. The entire code is written in standard $\mathrm{C}++$. A full description of the entire structure and implementation of the code is available in (Zhou 2012).

The main library that AD-GPRS uses to achieve this flexibility is an Automatic Differentiation Expression Template Library (ADETL) (Younis 2011; Zhou 2012). The computation of these automatic derivatives consists of: 1$)$ analyzing the expressions and to determine the basic operators $\left(+,-,{ }^{*}, /\right)$ that are involved; and 2) performing basic differentiation rules -- linearity, product, quotients, function derivatives and chain rule to evaluate the derivative. The key benefit that this provides is the ability to compute analytical derivatives automatically without any manual implementation of the Jacobian or by computing a numerical Jacobian. This manual implementation is often tedious and can be difficult to debug. Another alternative is to compute numerical derivatives by using a truncated Taylor series representation of the derivatives to represent the analytical derivatives. The main downside to this is that numerical derivatives lead to large truncation errors and small intervals would result in considerable round-off errors. A full description of this automatic differentiation library and implementation is available in (Younis 2011; Zhou 2012).

\subsection{CFRAC}

CFRAC (Complex Fracturing ReseArch Code) is a discrete fracture network code that fully implicitly couples fluid flow with the stresses induced by fracture deformation. The original version of the code was two-dimensional (McClure and Horne 2013), but recently the code has been extended to three dimensions (McClure et al. 2015). The fluid flow calculations are performed using the finite volume method (KarimiFard et al. 2004). Fluid leakoff from fractures into the matrix can be either neglected, handled with a onedimensional leakoff model (Vinsome and Westerveld 1980) or with a fully numerical solution using an unstructured conforming mesh (McClure 2014). Fluid is assumed to be single-phase, either gas or liquid (Ribeiro et al.). Calculations of stresses induced by fracture sliding and opening are performed with the constant boundary element method and the patch element formulation of Shou and Crouch (Shou and Crouch 1995) in 2D and Okada (Okada 1992) in 3D. The Shou and Crouch (Shou and Crouch 1995) method assumes the problem domain is an infinite whole space, but the Okada (Okada 1992) method 
assumes an infinite half-space. With the Okada (Okada 1992) method, deformations at the surface can be calculated.

When simulating fluid flow in the matrix surrounding the fractures, it is assumed that the deformations of the fractures do not poroelastically affect the fluid pressure in the matrix, and fluid pressure changes in the matrix do not affect the stress on the fractures. However, deformations of the fractures do have a poroelastic effect on the fluid pressure of other fractures. Nonlinear equations are used to relate fluid pressure, normal stress, and cumulative sliding displacement to the void and hydraulic aperture. The coefficient of friction can be assumed to be constant, or it can be assumed to be evolving according to a rate and state friction law. The code includes flow in both new and preexisting fractures, with the propagation of new fractures handled by numerically calculating a stress intensity factor at the crack tip. However, the location of any potentially forming hydraulic fractures must be specified in advance.

Similar to the fluid flow, thermal transport is simulated with the finite volume method. It is iteratively coupled to fluid flow. Thermal transport outside the fractures can be simulated with either a fully numerical simulation (in 2D) or a one-dimensional heat conduction model (in 2D or 3D). The onedimensional heat conduction model neglects the effect of effect of heat convection in the matrix. Thermoelastic effects are not included. The fully numerical treatment includes both convection and conduction.

\subsection{FALCON}

FALCON (Fracturing And Liquid CONvection) (Gaston et al. 2012a, b; Podgorney et al. 2010) is a fullycoupled and fully-implicit modeling tool for predicting the dynamics of fluid flow, heat and solute transport, geomechanics, and reactive geochemistry. The code is developed on a parallel Multiphysics Object Oriented Simulation Environment (MOOSE) (Gaston et al. 2009; Gaston et al. 2014a; Gaston et al. 2014b) computational framework developed at Idaho National Laboratory for providing finite element solutions of coupled systems of nonlinear partial differential equations using a Global Implicit Approach (GIA).

FALCON has been designed for the simulation of geothermal reservoirs, both conventional hydrothermal and EGS, but has been applied for multiphysics simulations of used fuel disposition of nuclear waste, groundwater flow and transport, and mining applications. The architecture of FALCON has a plug-andplay modular design structure based on representing each of the governing PDEs as a "kernel". Kernels may be coupled together in any number of ways to achieve different application goals - for any given simulation, any combination of kernels can be applied to make the problem as simple or complex as necessary. FALCON solves problems on structured or unstructured meshes, using standard finite element approaches for geomechanics and a stabilized finite volume approach for mass and heat transport. It was built to take advantage of modern HPC tools, such as adaptive mesh refinement, physics-based preconditioning, and advanced time stepping. Fig. 4-1 shows the basic architecture of FALCON, with the kernels at the uppermost level, directly underlain by the numerical framework and solver libraries used to couple the Kernels and perform reservoir simulations.

An auxiliary variable system has also been built into FALCON to handle solving most of the derived quantities and variables that are dependent on the primary kernels mentioned above. The number of auxiliary kernels needed for a given simulation depends on the choice of primary variables and whether they are formulated in terms of pressure-temperature or pressure-enthalpy. In general, a simulation run with the pressure-enthalpy formulation, considering geomechanical displacement and damaging, requires the most primary and auxiliary kernels and has the highest computational burden. 


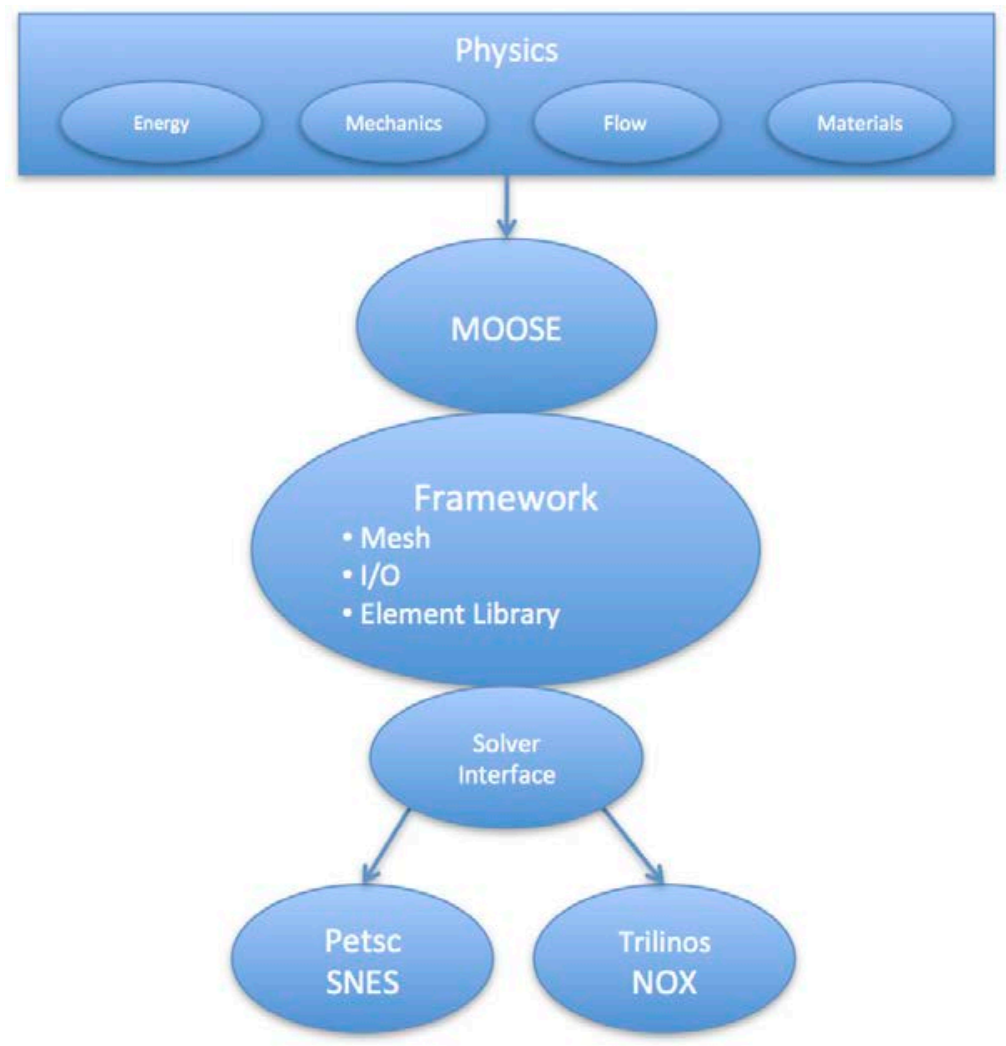

Figure 4-1. Architecture of the Falcon Code

\subsection{FEHM}

FEHM (Finite Element Heat and Mass) is a coupled simulator developed at the Los Alamos National laboratory (LANL) for modeling coupled thermo- hydro-mechanical and chemical (THM-C) processes in geomedia (Dempsey et al.; Kelkar et al. 2014; Kelkar et al. 2013; Pandey et al. 2014; Zyvoloski 2007). The simulator is designed to address spatial scales on the order of tens of centimeters to tens of kilometers, and time scales on the order of hours to tens of years. The coupled equations for fluid flow, mass transport and energy transport are implemented using finite volume, whereas the Galerkin finite element method is used for mechanical force balance. The governing coupled nonlinear equations are solved using a Newton-Raphson scheme with analytically or numerically computed Jacobians. A suite of models is available for coupling flow and mechanical deformation via permeability-deformation relationships.

The simulator FEHM was originally developed to model non-isothermal fluid flow in Hot Dry Rock geothermal reservoirs. FEHM has been developed extensively under projects on conventional/unconventional energy extraction (geothermal, oil, and gas), radionuclide and contaminant transport, watershed management, and $\mathrm{CO}_{2}$ sequestration. The capabilities of the simulator include nonisothermal, multiphase, multi-component fluid flow and chemical transport in media with dual porosity and dual permeability on unstructured grids. FEHM uses an efficient approach for evaluating thermodynamic functions using lookup tables and polynomial approximations to calculate derivatives. FEHM also uses a novel technique of decoupling the material coefficients from the geometric integrals involving shape functions. This allows the pre-computation of the geometric integrals at the beginning of the simulation, saving significant computational resources during iterative solutions of nonlinear 
problems. FEHM is capable of representing complex geometries using unstructured meshes to solve multi-phase flow of water, air and $\mathrm{CO}_{2}$.

The mass and energy balance equations are discretized using the control volume (CV) approach, as this is known to lead to good local mass conservation. The mechanical force balance equations are discretized with the finite element (FE) approach. Mapping between the CV and the FE grids is done such that the variables are defined at the same nodes. The mass and energy balance equations are solved simultaneously and are sequentially coupled to the force balance equation. Materials can display linear or nonlinear, elastic or elastic-plastic behavior, and may have anisotropic properties. Spatially varying material properties are mapped from nodes onto finite elements using shape function interpolations. Coupling between the fluid flow, heat transfer and mechanical force balance equations occurs both through the explicit occurrence of terms in balance equations (e.g., the effective stress and thermal stress), as well as through the dependence of various coefficients such as density, viscosity, permeability, and porosity on the state variables such as pressure, temperature, and stresses.

\subsection{FLAC $^{3 D}$}

$F L A C^{3 D}$ (Itasca Consulting Group 2014) is a three-dimensional explicit finite-difference program for engineering mechanics computation. The basis for this program is the well-established numerical formulation used by Itasca's two-dimensional program, FLAC (Itasca Consulting Group 2011). FLAC $C^{3 D}$ extends the analysis capability of FLAC into three dimensions, simulating the behavior of threedimensional structures built of soil, rock or other materials that undergo plastic flow when their yield limits are reached. Materials are represented by polyhedral elements within a three-dimensional grid that is adjusted by the user to fit the shape of the object to be modeled. Each element behaves according to a prescribed linear or nonlinear stress/strain law in response to applied forces or boundary restraints. The material can yield and flow, and the grid can deform (in large-strain mode) and move with the material that is represented. The explicit, Lagrangian calculation scheme and the mixed-discretization zoning technique used in $F L A C^{3 D}$ ensure that plastic collapse and flow are modeled very accurately. Because no matrices are formed, large three-dimensional calculations can be made without excessive memory requirements. The drawbacks of the explicit formulation (i.e., small time-step limitation and the question of required damping) are overcome by automatic inertia scaling and automatic damping that does not influence the mode of failure. $F L A C^{3 D}$ offers an ideal analysis tool for solution of three-dimensional problems in geotechnical engineering.

The mechanics of the medium are derived from general principles (definition of strain, laws of motion), and the use of constitutive equations defining the idealized material. The resulting mathematical expression is a set of partial differential equations, relating mechanical (stress) and kinematic (strain rate, velocity) variables, which are to be solved for particular geometries and properties, given specific boundary and initial conditions. An important aspect of the model is the inclusion of the equations of motion, although $F L A C^{3 D}$ is primarily concerned with the state of stress and deformation of the medium near the state of equilibrium. The inertial terms are used as a means by which to reach the equilibrium state in a numerically stable manner.

The method of solution in $F L A C^{3 D}$ is characterized by the following three approaches:

- finite difference approach (first-order space and time derivatives of a variable are approximated by finite differences, assuming linear variations of the variable over finite space and time intervals, respectively) 
- discrete-model approach (the continuous medium is replaced by a discrete equivalent - one in which all forces involved (applied and interactive) are concentrated at the nodes of a three-dimensional mesh used in the medium representation)

- dynamic-solution approach (the inertial terms in the equations of motion are used as numerical means to reach the equilibrium state of the system under consideration)

The laws of motion for the continuum are, by means of these approaches, transformed into discrete forms of Newton's law at the nodes. The resulting system of ordinary differential equations is then solved numerically using an explicit finite difference approach in time.

\subsection{GeoFrac-Mech}

GeoFrac-Mech is a two-dimensional numerical simulator which solves the coupled equations for fluid flow, heat transfer, stress, and fracture deformation for a single- or multi-fracture system. Aqueous fluid flow is considered within fractures according to Darcy's law, in response local changes in fracture permeability with local changes in fracture normal and shear deformation. Aqueous fluid flow between the fractures and surrounding rock is considered to be fluid leakoff. Heat transfer within the fracture is via conduction and convection, with thermal capacitance including a variable fracture aperture. Heat transfer between the fractures and surrounding rock is time and position dependent parameter. The simulator solves the thermo-poroelasticity equations, relating change in rock strain to changes in rock stress, fluid pressure and temperature, change in fracture volume to changes in rock stress, fluid pressure and temperature, via the boundary element solution (Ghassemi and Zhang 2006). A principal assumption of the boundary element method is that the fracture resides in an infinite homogeneous geologic media. For multiple fracture systems, where stresses induced by changes in temperature, fluid pressure, and deformations in other fracture segments are obtained through spatial and temporal superposition of the individual fracture segment solutions.

Deformation of the fracture is computed from changes in normal and shear stress on the fracture segments, yielding both normal and shear deformations. The response of rough fractures are modeled via the Barton-Bandis (Bandis et al. 1983) joint deformation model. Fracture slip is modeled to occur when the shear stress exceeds the shear strength of the fracture. A linear relation is used to determine the change in fracture aperture with the change in shear displacement. The joint is assumed to deform elastically until the stress conditions yield permanent slip, which is determined in accordance with the Mohr-Coulomb failure criterion. All equations are solved simultaneously, requiring iteration to resolve the nonlinearities. The temperature distribution and interface heat flow rate at every fracture segment can be solved for given fluid flow rate in fractures; and the fluid pressure, normal and shear fracture deformation, and interface flow rate can be solved for given interface heat flow rate. Solved together these equations yield the distribution of temperature, fluid pressure and fracture deformation.

\subsection{GeoFrac-Stim}

GeoFrac-Stim is a three dimensional numerical simulator for solving coupled thermal, hydrological, and mechanical processes in geologic media. The code solves three coupled equations for geomechanical displacement, fluid pressure and temperature using a finite-element formulation for discretization. The governing equations are the balance of force for geomechanics, continuity of fluid mass, and continuity of energy. A partitioned solution scheme is followed that splits the solution of the governing equations into two parts. First a prediction of the change in temperature is made, which is then used to solve the coupled equations for geomechanical displacement and fluid pressure. Fluid velocity is coupled with pore pressure variations via Darcy's law. Changes in temperature are then computed from the conservation of energy 
equation. Mechanical displacement considers impacts of fluid pressure and thermal expansion/contraction. Oscillations in the temperature solution due to thermal convection processes are dampened via the Streamline Upwind Petrov Galerkin stabilization scheme (Lee and Ghassemi 2011).

The simulator offers two approaches to modeling the geomechanical behavior of the rock: 1) thermoporoelastic and 2) damage and permeability. The thermo-poroelastic model considers the influence of fluid flow and temperature change on stress variation in the rock, but based on linear rock behavior without reaching the failure point. The damage and permeability model extends this capability to account for hardening and post-peak softening near the failure point, following the continuum damage mechanics theory, first introduced by (Kachanov 1958). A modified model of rock damage and permeability, proposed by (Tang et al. 2002) is used which considers continuous stress relaxation from the peak stress to the residual strength. This model divides the stress-strain relationship into elastic an damage phases. The elastic phase is reversible, but in the damage phase the rock begins to fail by crack initiation, crackgrowth, and void-growth beyond the failure criterion.

\subsection{GEOS}

GEOS is a massively parallel, multi-physics simulation application utilizing high performance computing (HPC) to address subsurface reservoir stimulation activities with the goal of optimizing current operations and evaluating innovative stimulation methods. GEOS enables coupling of different solvers associated with the various physical processes occurring during reservoir stimulation in unique and sophisticated ways, adapted to various geologic settings, materials and stimulation methods. Developed at the Lawrence Livermore National Laboratory (LLNL) as part of a Laboratory-Directed Research and Development (LDRD) Strategic Initiative (SI) project, GEOS represents the culmination of a three-year code development and improvement plan that has leveraged existing code capabilities and staff expertise to design new computational geosciences software (Settgast et al. 2014).

The overall architecture of the framework includes consistent data structures, generalized parallel communication and input/output functions, and interfaces for incorporating additional physics solvers and materials models as demanded by future applications. Along with predicting the initiation, propagation and reactivation of fractures, GEOS also generates synthetic micro-seismic source terms that can be used to generate motions at surface and down-hole array positions. GEOS can also be linked with existing, non-intrusive uncertainty quantification schemes to constrain uncertainty in its predictions and sensitivity to the various parameters describing the reservoir and stimulation operations.

GEOS development was originally motivated by the need to simulate hydraulic fracture stimulation; however, the capabilities are being expanded beyond this to include simulation of long-term fault behavior associated with injection-induced/triggered seismicity, modeling the behavior of discontinuous rock masses under load, particle method simulation of granular mechanics, flow and transport of heat and fluid in dual permeability (discontinuous/continuous) geologies, and numerical (finite difference based) propagation of seismic waves. GEOS can additionally call LLNL-proprietary material models and equations of state. Despite the expansion of its application space, the GEOS framework development continues to be focused on the solution of low-rate loading of coupled hydromechanical systems with the target application of better characterizing reservoir response to different stimulation, fracture control, and injection/disposal techniques over both stimulation and production timescales. 


\subsection{MULTIFLUX}

MULTIFLUX is a qualified, DOE-funded code developed for coupled thermal-hydrologic-air flow simulations for the proposed High-Level Nuclear Waste Repository at Yucca Mountain Project (Danko 2008). The coupling of the component codes is successfully accomplished using the Numerical Code Functionalization (NTCF) technique (Danko 2006), a transformative compression method that replaces the original component codes with their surrogate mathematical model by numerical functionalization. The component codes coupled in the conjugate solution use the MULTIFLUX Version 5.0 software as a framework for the coupled T-H-M-C model. MULTIFLUX is also used at the sub-model level as an integrated CFD solver for flow and transport simulations in the EGS fractures.

The NTCF model-elements for the rock mass and the CFD model-elements for the fracture are coupled by an iterative procedure in MULTILUX (Danko 2008). Fig. 4-2 is a solution flowchart showing the coupling process by the Direct Iteration and Successive Approximation coupler (DISAC) module. DISAC matches temperature heat flux, concentration, species flux, and bulk flow flux on the boundary surface node and time instance during simulation. The coupled simulation results are processed and saved by the DISAC module.

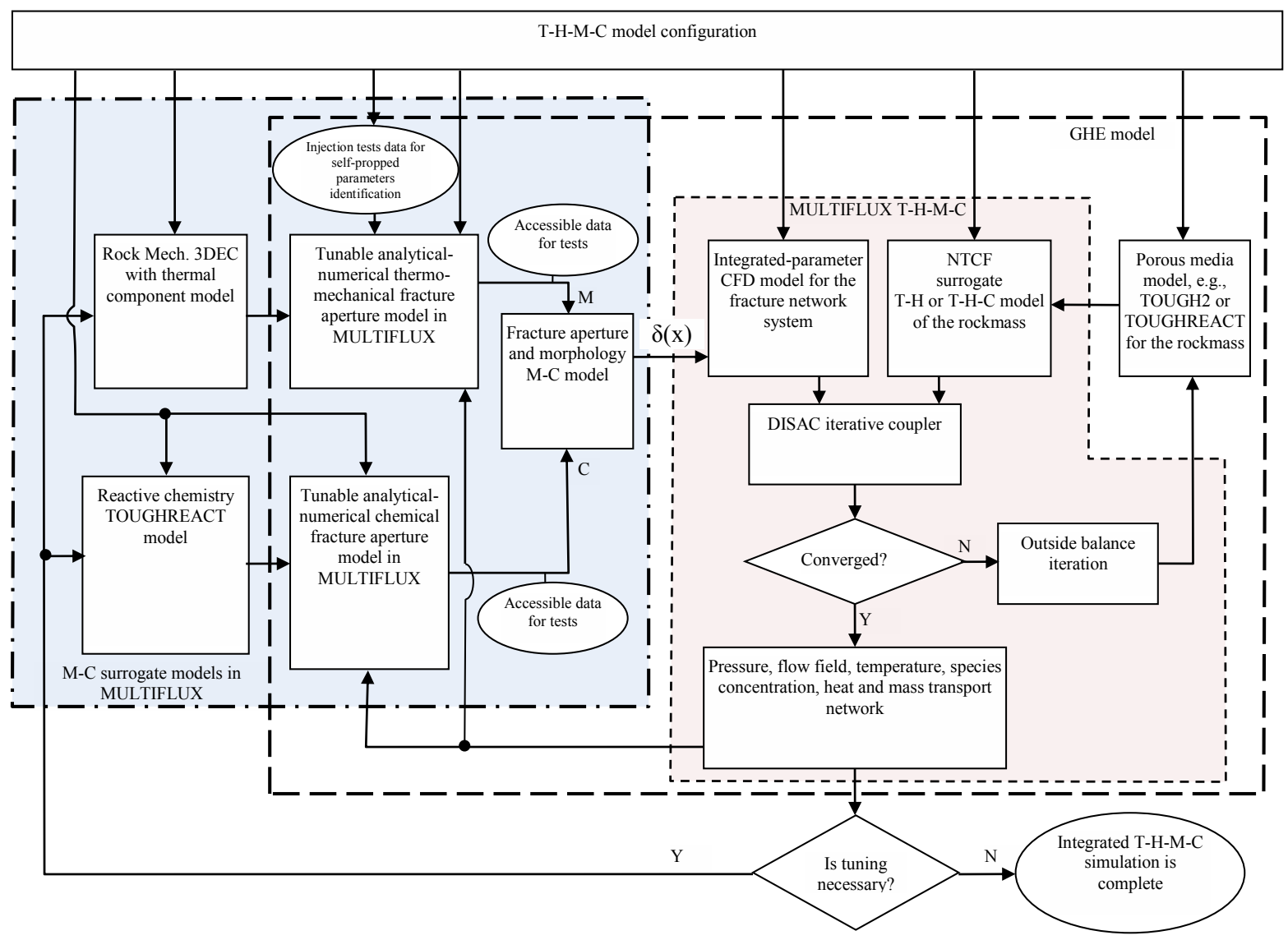

Figure 4-2. Logic flow chart of the new, integrated THMC model

Three iteration loops are used in the Internal Balance Iteration $(I B I)$ cycle to balance the rockmass and fracture flow transport processes, starting from the first, most inner loop to the third outer loop:

- Bulk flow calculation in the discretized fracture network system assuming an initial temperature, pressure and concentration distribution 
- Heat flow balance iteration between the NTCF and CFD models for each time division

- Chemical species mass flow balance iteration between the NTCF and fracture CFD models for each time division

The three iteration loops are executed until no significant change is observed in the results between consecutive iterations. While the CFD model-element is solved within MULTIFLUX, the NTCF modelelements are only surrogate models for the rockmass. TOUGH2 may be used as an independent solver for the heat and bulk coolant transport processes in the rockmass. TOUGHREACT may be used as an independent solver for the chemical species transport. In high temperature, strongly non-linear applications, the NTCF model parameters must be refreshed during coupling iterations (Danko 2006).

\subsection{NUFT}

The NUFT (Nonisothermal Unsaturated-saturated Flow and Transport) code is a highly flexible computer software package for modeling multiphase, multi-component heat and mass flow and reactive transport in unsaturated and saturated porous media. This computer code has been developed over decades at the Lawrence Livermore National Laboratory (LLNL), and represents a current state-of-the-art in subsurface flow and reactive transport modeling (Hao et al. 2013; Nitao 2000). NUFT uses an integral finite difference spatial discretization along with implicit time-integration schemes to solve the mass and energy balance equations. The resulting non-linear equations at each time step are solved by the NewtonRaphson method. Several models or modules, which utilize a common set of utility routines and input file formats, are implemented in NUFT to address various flow and reactive transport processes in porous media. Available modules include:

- UCSAT - unconfined and confined aquifer, saturated flow module

- US1P - unsaturated single phase flow module (Richard's equation)

- USNT - fully coupled unsaturated multiple phases, multiple components flow module with isothermal and nonisothermal options

- US1C - unsaturated single component transport module

- TRANS - geochemical multiphase transport module

- RESHEAT - electrical resistive heating module

These modules can either work as stand-alone models or be coupled with another sub-model through internal inter-module data exchange/transfer.

NUFT is capable of running on PCs, workstations, and major parallel computing platforms. It has been widely used for numerical modeling of subsurface multiphase flow and reactive transport processes. Application areas include geological disposal of nuclear waste, $\mathrm{CO}_{2}$ geologic sequestration and storage, geothermal exploitation, groundwater monitoring and remediation, and subsurface hydrocarbon production.

\subsection{STOMP}

STOMP (Subsurface Transport Over Multiple Phases) (STOMP User Guide 2015; White and Oostrom 2000; White et al. 2012; White and McGrail 2005; White and Oostrom 2006) is a suite of numerical simulators for modeling multi-fluid flow, heat transport, and reactive transport in geologic media. The suite of simulators is distinguished by operational modes that solve various combinations of conservation equations and associated constitutive equations, including the equations of state. Fluid flow is computed using a multifluid version of Darcy's equation and diffusive/dispersive transport is considered in all 
fluids. Phase appearances, disappearances, and transitions are computed dynamically depending on the component composition, temperature and pressure state, assuming thermodynamic equilibrium. The heat transport and component mass conservation equations are solved implicitly using a Newton-Raphson scheme with numerically generated derivatives. Spatial discretization is via an integral volume formulation on structured hexahedral grids and temporal discretization is via backward Euler.

Reactive transport is solved sequentially to the coupled heat transport and component mass conservation equation using an operator-splitting scheme where local geochemistry is solved following transport of conserved and kinetic species. Reactive processes include mineral precipitation/dissolution, gas exsolution/dissolution, ion exchange, and surface complexation via the solution of three general reaction equation types: 1) equilibrium, 2) conservation, and 3) kinetic, using the ECKEChem (Equilibrium, Conservation, Kinetic Equation Chemistry) module. Newton-Raphson iteration is used to resolve the nonlinearities in the reaction equations. The precipitation and dissolution of minerals is coupled to porosity and intrinsic permeability that then affect the coupled fluid flow and heat transport. For geomechanical calculations, STOMP has been solved sequentially with SIMULIA's Abaqus for carbon sequestration applications. For this study, geomechanical changes in fracture aperture and associated permeability were modeled assuming static stress conditions in the rock, yielding a mean effective stress as a function of pressure only.

The development of the STOMP simulator was initiated in the early 1990s with the investigations of the technical potential for Yucca Mountain as a nuclear waste repository (Eslinger et al. 1993). STOMP's capabilities were soon expanded to study the migration of volatile organics through the deep vadose zone at the Hanford Site (Lenhard et al. 1995; White et al. 1995). Since these early development stages, application areas for the simulator have greatly expanded to include environmental remediation (Oostrom et al. 2003; Zhang et al. 2012), vegetated surface barriers (White and Ward 2005), carbon sequestration in deep saline reservoirs (Cheng et al. 2013), the production of oil and gas from oil shales, enhanced oil recovery with carbon dioxide injection (White et al. 2014b), compressed gas storage (McGrail et al. 2013), storage of low-level nuclear waste in glass form (McGrail et al. 2001), production of natural gas hydrates (White and Lee 2014), and geothermal (White and Phillips 2015). Parallelization of the simulator varies across the operational modes, from sequential only, to threaded via OpenMP and coupling with PETSc, to fully parallel on distributed memory computers via Global Arrays, MPI, and PETSc.

\subsection{TOUGH2 and TOUGH-FLAC}

Developed at the Lawrence Berkeley National Laboratory since the early 1980 s, TOUGH 2 is currently the most widely applied code in the TOUGH suite of nonisothermal multiphase flow and transport simulators (Finsterle et al. 2014; Pruess et al. 2011). TOUGH2, through its predecessors MULKOM (Pruess 1983) and TOUGH (Pruess 1987), was initially developed primarily for geothermal reservoir engineering, but is now widely used for applications related to geological carbon sequestration, nuclear waste disposal, energy production from geothermal, oil and gas reservoirs as well as methane hydrate deposits, environmental remediation, vadose zone hydrology, and other uses that involve coupled thermal, hydrological, geochemical, and geomechanical processes in permeable media (Finsterle et al. 2014). The basic TOUGH2 code considers flow of multiphase fluid mixtures and heat in fractured porous media for select components and phases. The considered combination of components and related fluid properties are calculated by the particular EOS fluid property module that is linked to the main flow and transport core, which sets up and solves the mass-and energy-balance equations. An unstructured finite-volume (integrated finite-difference) numerical approach is implemented for the flow/transport computations. This allows modeling of porous and/or fractured systems and other types of systems with zones of different component mobility, through the use of multiple interacting continua (MINC) (Pruess and 
Narasimhan 1985). The TOUGH suite of simulators, including TOUGH2 have over the years been continually updated, with new equation-of-state (EOS) modules, and refined process descriptions, and the numerical performance of the simulators is being improved through parallelization and the use of state-ofthe-art linear equation solvers (Finsterle et al. 2014). All EOS modules contain water, and may include non-condensable gases, solutes, volatile organic compounds, tracers, and radionuclides. These components are transported through the geologic media in one, two, or three fluid phases (gaseous, aqueous, and non-aqueous phase liquids). Some components may also precipitate and dissolve, or adsorb and desorb, i.e., become part of or interact with an immobile solid phase. The main fluid flow processes considered in the basic TOUGH simulators are advective fluid flow described with a multiphase extension of Darcy's law, which includes relative permeability and capillary pressure effects; in addition, there is diffusive mass transport in all phases. Heat flow occurs by conduction and convection as well as sensible and latent heat effects (Finsterle et al. 2014; Pruess et al. 2011).

TOUGH-FLAC is a simulation approach based on linking TOUGH2 and FLAC ${ }^{3 \mathrm{D}}$, originally developed during the late 1990s for considering geomechanical effects on multiphase flow and transport associated with the Yucca Mountain nuclear waste disposal project (Rutqvist and Tsang 2003; Rutqvist et al. 2002). TOUGH-FLAC is currently being applied to study coupled geomechanical processes under multiphase flow conditions for a wide range of applications, including nuclear waste disposal (Rutqvist et al. 2014b), $\mathrm{CO}_{2}$ sequestration (Rutqvist et al. 2007; Rutqvist et al. 2014a), EGS (Rutqvist et al. 2015a), gas production from hydrate-bearing sediments (Rutqvist and Moridis 2009), and compressed air energy storage (Rutqvist et al. 2012). TOUGH-FLAC is currently being extensively applied for studies related to injection-induced fault activation and seismicity associated with $\mathrm{CO}_{2}$ sequestration (Rutqvist et al. 2014a) stimulation of shale-gas reservoirs (Rutqvist et al. 2015b), and EGS (Jeanne et al. 2014). Other efforts of TOUGH-based geomechanical models have also been developed, such as for modeling of fracture propagation of various complexity, including TOUGH-RDCA (Pan et al. 2014) and TOUGH-RBSN (Asahina et al. 2014), though those have not yet applied for the analysis of EGS.

In the TOUGH-FLAC approach, the two codes TOUGH2 and FLAC ${ }^{3 \mathrm{D}}$ are sequentially coupled through a central THM model that is material specific and depends on the application (Fig. 4-3). The couplings include direct pore-volume coupling between mechanics and hydraulics, as well as indirect coupling through property changes. For example, permeability and capillary scaling can be dependent on mechanically induced porosity changes. For fractured media, various models have been applied to correct permeability for changes in the three-dimensional stress field (Rutqvist et al. 2002). Faults and discontinuities that can be discretized either using FLAC ${ }^{3 \mathrm{D}}$ interfaces or finite thickness elements with strongly anisotropic properties (Rutqvist et al. 2007; Rutqvist et al. 2015a). Models involving Bandis' normal closure model and strain-hardening-softening shear behavior have also been used for modeling fractures and faults, including strain-softening (slip weakening) shear strength applied for modeling sudden (seismic) fault slip (Rutqvist et al. 2014a). Numerially, the coupling of TOUGH and FLAC ${ }^{3 \mathrm{D}}$ corresponds to a mixed formulation that is stable in space, whereas the sequential coupling scheme uses so-called stress fixed iterations that are known to result in an unconditionally stable solution (Kim et al. 2012; Kim et al. 2011). The fixed stress iterations means that the flow problem is solved first under fixed stress, whereas pressure, temperature are prescribed during the mechanical run and porosity in the flow simulation is updated through a special correction scheme (Kim et al. 2012; Kim et al. 2011).

Related to EGS, TOUGH-FLAC was the main code used for planning, design, and validation of the stimulation operation associated with The Geyser EGS Demonstration Project in California (Rutqvist et al. 2015a) and also has also been used for modeling shear stimulation at the Newberry Volcano EGS Demonstration (Rinaldi et al. 2015). Both cases involve relative cool water injection into very hot (up to $350^{\circ} \mathrm{C}$ ) reservoir rock, associated stress changes, and induced seismicity. In the case of the Geysers, a network of discrete fracture zones were discretized into the 3D model of the site and these have a profound effect on the coupled THM behavior of the EGS (Jeanne et al. 2014). 


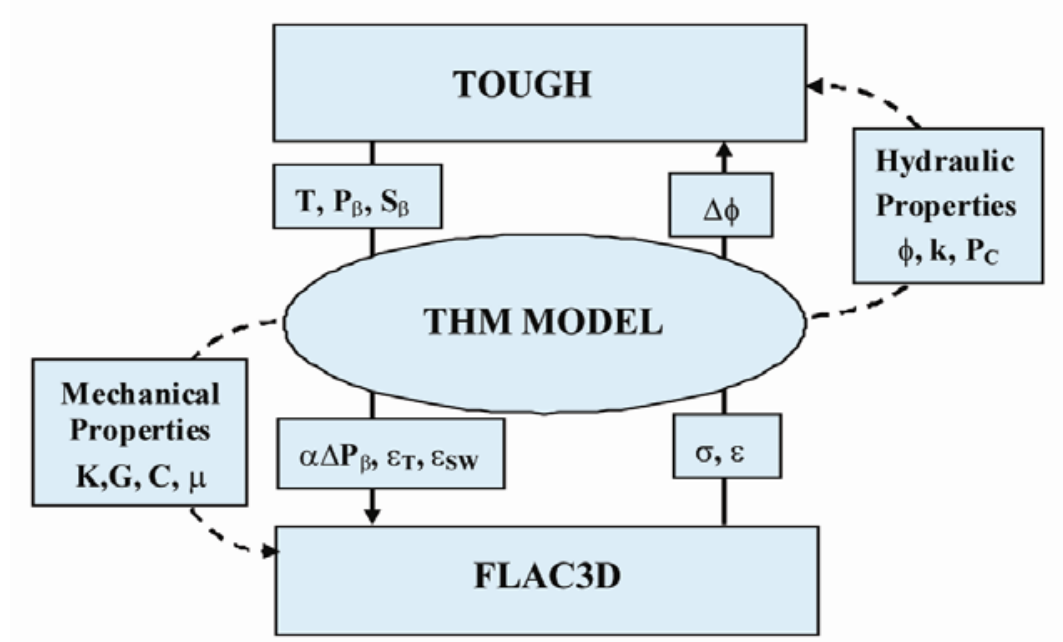

- Direct couplings
-- Indirect coupling
$\mathrm{C}=$ Cohesion
$\mathrm{G}=$ Shear modulus
$\mathrm{K}=$ Bulk modulus
$\mathrm{k}=$ Intrinsic permeability
$\mathrm{P}_{\beta}=$ Pressure of phase $\beta$
$\mathrm{P}_{\mathrm{c}}=$ Capillary pressure
$\mathrm{S}_{\beta}=$ Saturation of phase $\beta$

\begin{tabular}{|l|}
\hline \\
$\varepsilon=$ Temperature \\
$\varepsilon=$ Strain \\
$\varepsilon_{\mathrm{T}}=$ Thermal strain \\
$\varepsilon_{\mathrm{S} w}=$ Swelling strain \\
$\alpha=$ Biot's parameter \\
$\phi=$ Porosity \\
$\mu=$ Coefficient of friction \\
$\sigma=$ Stress
\end{tabular}

Figure 4-3. Schematic of linking of TOUGH2 with FLAC ${ }^{3 \mathrm{D}}$ for a TOUGH-FLAC coupled THM simulation (Rutqvist 2011)

\subsection{TOUGHREACT and TOUGHREACT-ROCMECH}

Reactive transport in geothermal systems is inherently a multicontinuum problem, typically characterized by a more permeable fracture "network" in communication with a porous lower permeability rock matrix. Whereas fluid flow and heat transport can often be simulated adequately in "equivalent" or singlecontinuum media, large differences in fracture and matrix mineralogy, fluid chemistry, and potentially gas saturation, make it imperative to consider multiple interacting continua (MINC) as extended to water-gasrock interaction(Sonnenthal et al. 2005; Xu and Pruess 2001; Xu et al. 2001). While such methods are computationally intensive in 3D, accurate models of THMC processes on reservoir-scale problems will require such methods. TOUGHREACT (Sonnenthal et al. 2014; Xu and Pruess 2001; Xu et al. 2006; Xu et al. 2001; Xu et al. 2011) was developed by introducing reactive geochemistry into the framework of TOUGH2, for which the multiple continuum approach had been well developed for fluid and heat flow.

TOUGHREACT simulates non-isothermal, multiphase, multicomponent reactive transport of aqueous and gaseous components in porous and fractured media. Reactive transport is solved by an operatorsplitting approach that can be either iterative or non-iterative. The code can accommodate an arbitrary number of chemical species that can be present in liquid, gas and solid phases in media with physical and 
chemical heterogeneity. Thermophysical and geochemical properties are calculated as a function of pressure and temperature as well as of thermodynamic and kinetic data for mineral-water-gas reactions. Multiphase transport of aqueous and gaseous species occurs by advection and molecular diffusion. Aqueous and surface complexation, acid-base, redox, gas dissolution and exsolution, and multi-site cation exchange are considered under the local equilibrium assumption. Mineral dissolution and precipitation proceed under either equilibrium or kinetic constraints. Intra-aqueous kinetics and biodegradation and surface complexation using non-electrostatic, constant capacity and double layer electrostatic models were incorporated into TOUGHREACT V2 (Xu et al. 2011).

Mineral dissolution and precipitation lead to temporal changes in porosity and associated changes in permeability and unsaturated hydrologic properties, which is an important feedback mechanism that modifies fluid flow patterns. The porosity-permeability correlation in geologic media can be complex, depending on factors such as pore size distribution, pore shape, connectivity, and crystal morphology. Several porosity-permeability and fracture aperture-permeability, and capillary pressure-porositypermeability relationships are included (Sonnenthal et al. 2005; Sonnenthal et al. 2014). TOUGHREACT has been applied to a wide range of subsurface hydrological and biogeochemical environments, including applications related to geothermal systems, nuclear waste repositories, geologic carbon sequestration, and environmental remediation (Aradóttir et al. 2012; Dobson et al. 2004; Finsterle et al. 2014; Sonnenthal et al. 2005; Sonnenthal et al. 2012; Sonnenthal et al. 2015; Sonnenthal et al. 2014; Spycher et al. 2003; Xu et al. 2004a; Xu et al. 2004b; Xu and Pruess 2001, 2004; Xu et al. 2001).

TOUGHREACT Version 3.0-OMP (Sonnenthal et al. 2014) adds many new features for simulating 3D EGS and hydrothermal systems, including OpenMP parallelization of the most time-consuming routines. Composition and temperature-dependent mineral heat capacities and thermal conductivities calculated from thermodynamic data can be dynamically updated during mineral precipitation-dissolution, and the coefficients calculated directly from mineral abundances for use in $\mathrm{TH}(\mathrm{M})$ calculations. Homogeneous and heterogeneous heats of reaction can be calculated from the thermodynamic database and coupled to heat and reactive transport. Reactive trace gas species can be injected/produced and transported with a $\mathrm{CO}_{2}-\mathrm{H}_{2} \mathrm{O}$ "carrier" gas. A newly developed single phase (aqueous) nonisothermal wellbore flow model can be easily implemented with little modification to existing reservoir models.

Ongoing development of TOUGHREACT includes hybrid MPI-OpenMP parallelization for large-scale parallel computing, new equation-of-state modules, and coupling of geomechanical effects in the new THMC(B) simulator TOUGHREACT-ROCMECH.

TOUGHREACT-ROCMECH (Kim et al. 2015; Kim et al. 2012) is one of several codes that have been developed based on TOUGHREACT to consider aspects of coupled THMC processes. ROCMECH was developed specifically for treating stimulation in EGS, providing the ability to more tightly couple THC and mechanical processes, without using an external "black-box" simulator. TOUGHREACT-

ROCMECH considers changes in pore volume, induced by chemical reaction, poroelasticity, tensile, and shear failure when solving for fluid and heat flow (Kim et al. 2015). The fluid and heat flow, geomechanics, and reactive transport are solved sequentially, adopting implicit solution schemes for the sub problems (i.e., sequential implicit method), as shown in Fig. 4-4. Specifically, when solving flow and geomechanics, the fixed stress sequential method was employed, which solves flow first, fixing the total stress field locally, and solves geomechanics at the next step from the calculated flow solutions. As discussed above, the fixed-stress method can provide numerical unconditional stability and high accuracy, comparable to the fully implicit method, regardless of the coupling strength in pore-volume between flow and geomechanics (Kim et al. 2012; Kim et al. 2011). For EGS, TOUGHREACT-ROCMECH has been extended to coupled THMC in multiple porosity materials, including thermoporoplasticity for considering tensile and shear failure. Recently, the code has been has been further improved to solve wellbore stimulation problems in EGS and unconventional hydrocarbon reservoirs (Smith et al. 2015). Detailed 
injection-flowback 3D THM simulations of the stimulation at the Newberry Volcano EGS Demonstration have been performed, considering shear failure along multiple planes that represent the dominant fracture orientations at the site (Smith et al. 2015; Sonnenthal et al. 2015).

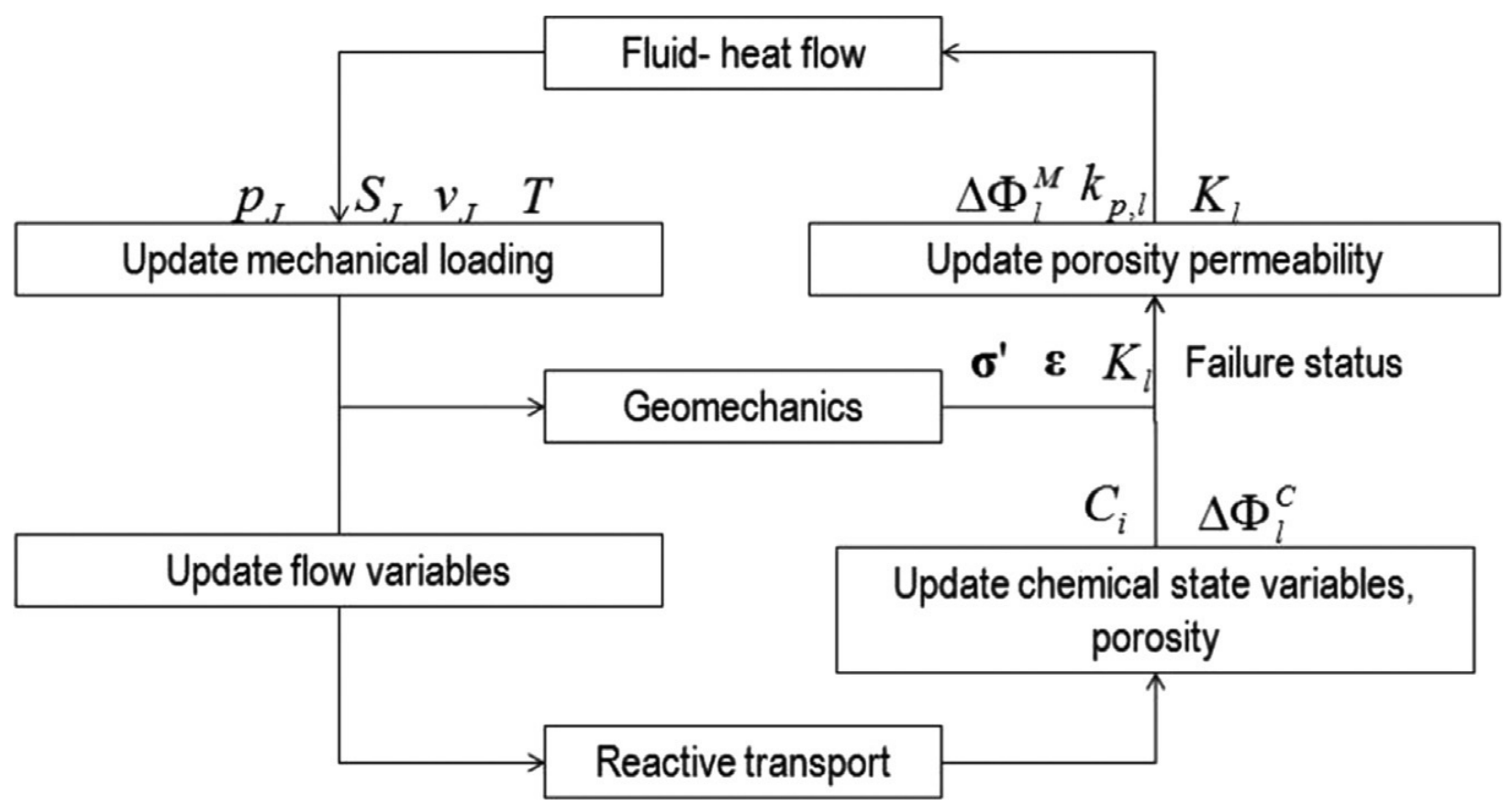

Figure 4-4. The sequential implicit algorithm of chemo-thermo-poro-mechanics. Flow, geomechanics, reactive transport simulators are communicated sequentially (Kim et al. 2015)

\subsection{TR_FLAC3D}

TR_FLAC3D (Taron and Elsworth 2009, 2010b) is a continuum model that represents coupled THMC interactions in dual porosity media including undrained loading. Equilibrium and complex mechanical constitutive relations are enforced in FLAC ${ }^{3 \mathrm{D}}$. Transport of fluid (Darcian), dissolved mineral mass (Fickian and advective) and heat (conductive and advective) are accommodated in TOUGHREACT together with non-equilibrium reactions. Primary dependent variables of displacement and fluid pressure in FLAC ${ }^{3 \mathrm{D}}$ and of pressure, aqueous concentration and temperature in TOUGHREACT are mapped between different grid structures in the two codes. Undrained loading is accommodated in FLAC ${ }^{3 \mathrm{D}}$. Undrained pressures are calculated from dual porosity constitutive relations in FLAC $^{3 \mathrm{D}}$ and interpolated, then redistributed in TOUGHREACT. Revised pressures are returned to FLAC ${ }^{3 \mathrm{D}}$, then incremented by undrained pressures that honor total stresses (equilibrium) in $\mathrm{FLAC}^{3 \mathrm{D}}$. This principal mode of interaction is illustrated Fig. 4-5.

Thermal behavior includes dual porosity response with thermal capacitance in the conductive matrix and advective transport in fractures. This is coupled with linear thermal expansion in the matrix and saturation weighted densities, specific heats and conductivities. Hydraulic behavior is also dual porosity for multiphase flow with matrix permeabilities weighted by saturation and fracture permeabilities controlled by stress. Mechanical behavior uses a cracked continuum with dual porosity deformation response (Bai and Elsworth 2000; Elsworth and Bai 1992) comprising a linear elastic matrix but nonlinear elastic fracture with exponential closure to residual aperture, damage upon closure limiting recovery, and linear dilation in shear following a Mohr-Coulomb failure criterion. The deformation cycle includes the generation of undrained fluid pressures and of thermal stresses, each in dual porosity media (Bai and 
Elsworth 2000; Elsworth and Bai 1992).

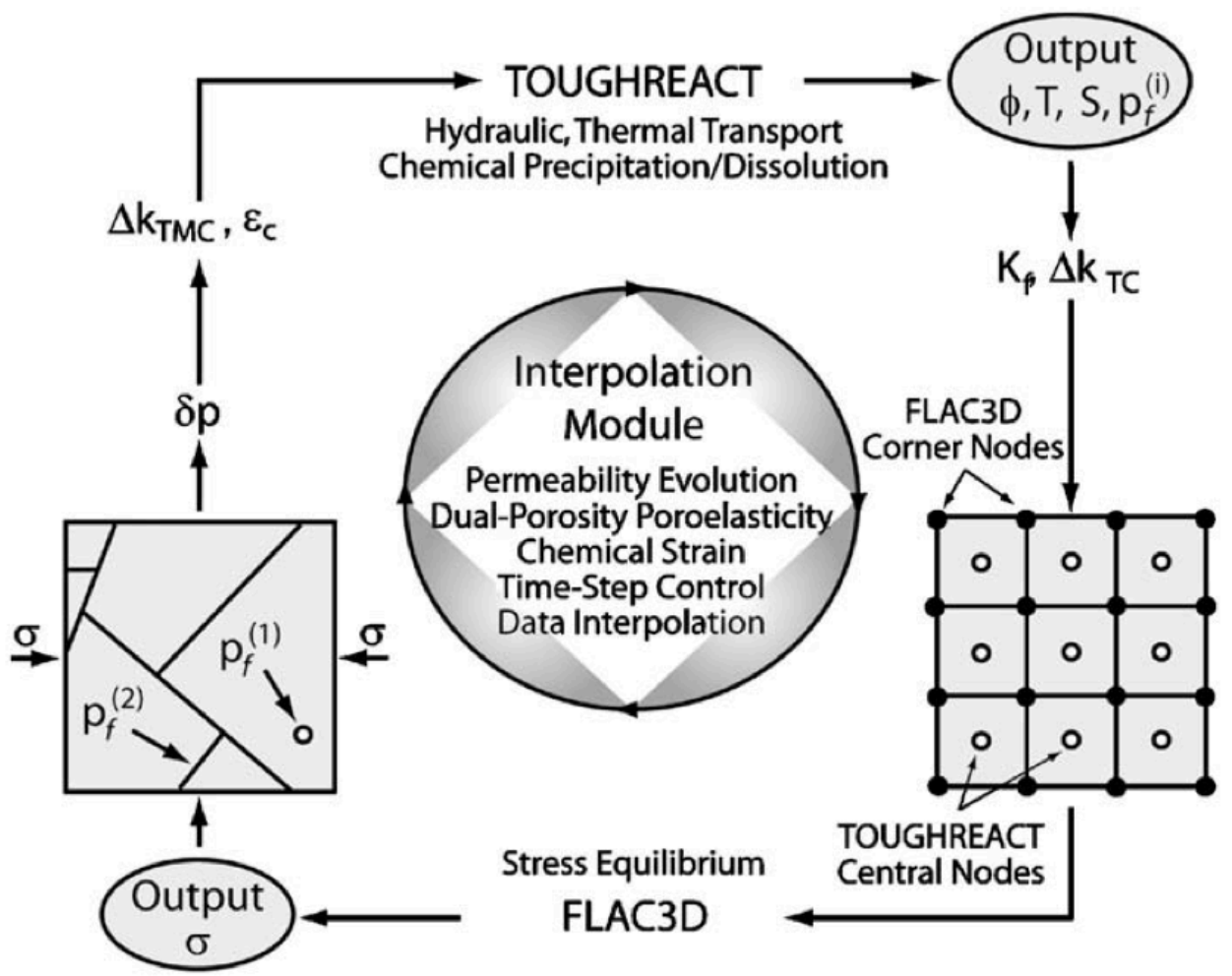

Figure 4-5. Coupling relationship between TOUGHREACT, FLAC ${ }^{3 \mathrm{D}}$ and the interpolation module, illustrating the different grids used in the two principal models

Chemical response incorporates dual porosity transport together with dissolution/precipitation that is rate constant dependent for multiple species and with Arrhenius dependence. THMC coupling accommodates fracture closure and permeability evolution due to TMC effects including dissolution and precipitation on free faces of fractures and dissolution from bridging asperities. This conforms to both equilibrium closure with pressure solution activated by a critical stress and with equilibrium dissolution (Min et al. 2009) and also disequilibrium closure with non-equilibrium dissolution (Taron and Elsworth 2010a) with Boussinesq compaction of asperities and rate-controlled dissolution.

\subsection{UDEC Universal Distinct Element Code}

The UDEC and 3DEC codes are used to analyze coupled thermo-hydro-mechanical processes in discontinuous media via the distinct element method (DEM) (Itasca Consulting Group 2013). Using the distinct element method to solve discrete geomechanics problem is described in the previous section. Several coupled fluid flow models are part of UDEC and 3DEC. The transient compressible joint flow model is the most applicable to geothermal reservoir analysis and is described here. In this formulation matrix flow is not considered. Fluid flow domains (locations where fluid pressure is defined) exist between the mechanical contact points which represent rock joints. A length is associated with each mechanical contact. The rate of fluid flow, q, across a contact (from one domain to another) is given by,

$$
q=-k_{j} a^{3} \frac{\Delta p}{l}
$$


where, $k_{j}$ is the joint permeability factor (whose theoretical value is $1 / 12 \mu$, where $\mu$ is the fluid dynamic viscosity), $a$ is the joint hydraulic aperature, $\Delta p$ is the difference in fluid pressure between adjacent domains and $l$ is the contact length. Because the distinct element method uses the soft-contact approach in which adjacent blocks are allowed to interpenetrate the hydraulic aperture of a joint is not directly represented. Joint hydraulic aperture is defined as

$$
a=a_{0}+u_{n}
$$

where, $a_{0}$ is the aperture at zero normal stress and $u_{n}$ is the joint normal displacement described above. The apertures can be bound by user specified upper and lower limits.

During each time step, new block positions are found which may result in joint hydraulic aperture or fluid domain volume change. The domain fluid pressure is update as

$$
p=p_{0}+K_{w} Q \frac{\Delta t}{V}-K_{w} \frac{\Delta V}{V_{m}}
$$

where $p$ and $p_{0}$ are the new and old fluid pressures, $K_{w}$ is the fluid bulk modulus, $\Delta V$ is the domain volume change, $Q$ is the unbalanced flow into the domain and $V_{m}$ is the average of the old and new domain volumes. These fluid pressures are applied to the adjacent blocks to achieve two-way hydromechanical coupling. Total stresses result inside the blocks and effective stresses are obtained at the mechanical contacts. Standard Neumann and Dirichlet boundary conditions can be applied in this scheme. Additional terms can be included to account for partial saturation, two phase flow, or gas flow. A standard vertex centered finite volume method is used to solve the transient heat conduction equation over the grid points. The resulting temperature can influence block and joint stresses and fluid properties. Advection of heat and convective heat transfer terms can also be included in this approach. 



\title{
5.0 Benchmark Problem 1: Poroelastic Response in a Fault Zone (Permeability-Pressure Feedback)
}

\author{
Problem Champion: Robert Podgorney, Idaho National Laboratory
}

Benchmark Problem 1 was loosely based on recent observations at a test well from the Raft River EGS demonstration site in southern Idaho (Bradford et al. 2013; Bradford et al. 2015; Huang et al. 2013). However, the data were simplified and generalized for the purposes of the code comparison study. The simulation problem involved a poroelastic fault layer in a geothermal reservoir undergoing water injection. The observed reservoir behavior includes a strong non-linear response between the injection rates and pressures over multiple-day, variable-rate injection tests. Simulation of the behavior is simplified with the assumption of the permeability being an exponential function of hydrodynamic pressure in the inferred fault zone. Water was injected into a well at a constant rate of $80 \mathrm{~kg} / \mathrm{s}$, with the fluid entering the reservoir in a narrow (4-meter thick) fault zone at a depth of $2000 \mathrm{~m}$. Simulated pressure and temperature are given in the wellhead over a 3 day test, emulating monitored data. For simplicity, the injected fluid is assumed to be at the virgin rock temperature of the reservoir at $140 \mathrm{deg} C$, minimizing the heat transport between the coolant fluid and the host rock. Participants could choose whether or not to model the overlying reservoir rock, with two sets of results being presented for either a case with no leak off to the dense formation and a case with leak off. Eleven teams participated in the solutions using various simplifying assumptions, multiple runs and THM models as listed in Table 5-1.

Table 5-1. Participating Teams and Simulators Used in the Solution of Benchmark Problem 1

\begin{tabular}{lll}
\hline Simulation Team & Team Identifier & Code(s) \\
\hline Idaho National Laboratory & INL & FALCON \\
Itasca Consulting Group & Itasca & FLAC $^{3 \mathrm{D}}$ \\
Lawrence Berkeley National Laboratory & LBNL & TOUGH and FLAC \\
Lawrence Livermore National Laboratory & LLNL & GEOS \\
Oak Ridge National Laboratory & ORNL & PFLOTRAN \\
Pennsylvania State University & PSU & FLSC3D \\
Pacific Northwest National Laboratory & PNNL & STOMP \\
Stanford University & Stanford & CFRAC and AD-GPRS \\
University of Nevada, Reno & UNR & MULTIFLUX and \\
& & TOUGH2 \\
The University of Oklahoma & OU & GEOFRAC \\
The University of Texas at Austin & UTA & CFRAC_UT \\
\hline
\end{tabular}

\subsection{Geometry and Input Data for Benchmark Problem 1}

The schematic of the arrangement is shown in Fig. 5-1(a). The domain may be considered in Cartesian or cylindrical coordinates depicted in Figs. 5-1(b) and (c), respectively. A disc-shaped fracture of in cylindrical coordinates is the most economical. In this case, the injection point is at the center of the hollow disk at radius $0.15 \mathrm{~m}$ at $2000 \mathrm{~m}$ depth. The outer perimeter of the disk is at $2828.42 \mathrm{~m}$, modeling the hypotenuse of a square of $2000 \mathrm{~m}$ sides. In Cartesian domain, the bottom, left, front and top-sides are treated as no flow and zero heat flux boundaries for simplicity. The right- and back-sides are at fixed fluid pressure and temperature with values equal to the initial fluid pressure and temperature in the reservoir. 


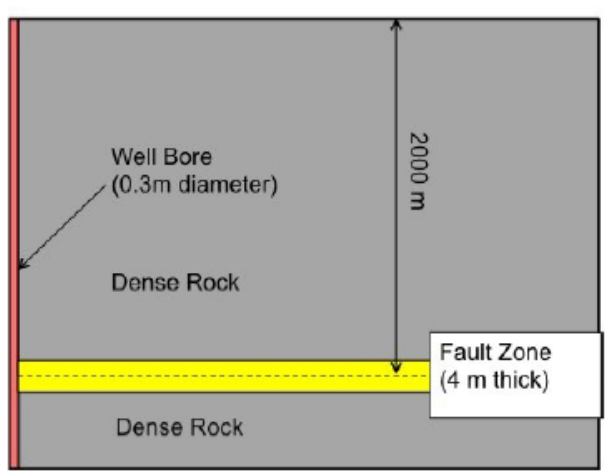

(a) Schematic

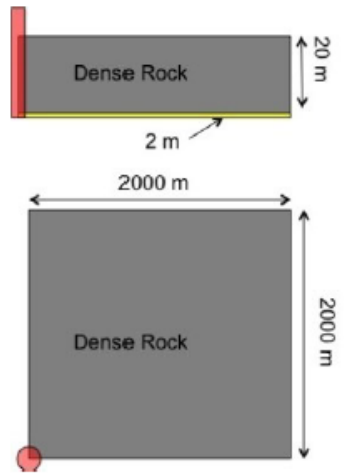

(b) Cartesian

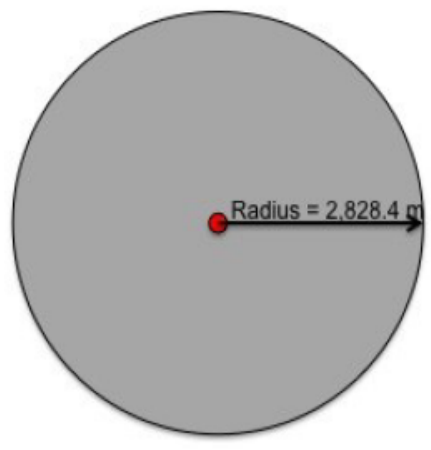

(c) Radial

Figure 5-1. Conceptual model for Benchmark Problem 1

A 1-dimensional cylindrical grid is recommended. A wedge of the complete cylindrical domain may be modeled in Cartesian coordinates. The density of discretization of the domain is as desired by individual participants, using e.g., 100 grid cells in the radial direction with geometrically increasing grid spacing, starting with a grid spacing of $0.05 \mathrm{~m}$. Other choices may be used with geometrical progression which is fine at the injection point and gradually increases according to

$$
r(i)=0.15+\sum_{j=1}^{i} q^{j} ; \text { with } q>1
$$

where $q$, the quotient, varies for representing finer or coarser grids as needed to check grid-independency. The results should be reported at grid centroids at $1.414 \mathrm{~m}, 3.535 \mathrm{~m}, 7.071 \mathrm{~m}, 14.14 \mathrm{~m}, 70.71 \mathrm{~m}$, and $707.10 \mathrm{~m}$, to meet the reporting requirements in Cartesian grids at $\mathrm{x}=1.0 \mathrm{~m}, \mathrm{y}=1.0 \mathrm{~m} ; \mathrm{x}=2.5 \mathrm{~m}, \mathrm{y}=2.5$ $\mathrm{m} ; \mathrm{x}=5.0 \mathrm{~m}, \mathrm{y}=5.0 \mathrm{~m} ; \mathrm{x}=10.0 \mathrm{~m}, \mathrm{y}=10.0 \mathrm{~m} ; \mathrm{x}=50 \mathrm{~m}, \mathrm{y}=50 \mathrm{~m}$; and $\mathrm{x}=500 \mathrm{~m}, \mathrm{y}=500 \mathrm{~m}$, respectively. Only the fractured zone is considered in the no leak-off case in simulation, setting the vertical height of the computational domain to $4.0 \mathrm{~m}$. In the leak-off case, Darcy flow in the compressible dense rock may be considered.

The effective-stress-dependent permeability characteristics of the fracture/fault zone are assumed to follow the exponential law of (Nathenson 1999):

$$
k=k_{o} \exp \left(\frac{c\left[P-P_{o}\right]}{\sigma}\right)
$$

where $k_{0}$ is the permeability at zero stress, the exponent $c$ is a fitting parameter that can be experimentally determined, $P$ and $P_{o}$ are the current and initial fluid pressures (in $\mathrm{MPa}$ ), and $\sigma$ is the total overburden confining stress (in MPa). Table 2 summarizes the parameters for the permeability function. Examination of the permeability function reveals that the permeability response can be determined using the fluid pressure alone, and that calculations of effective stress are not required. This was chosen for initial ease of comparison, and to allow codes that do not include mechanics the ability to participate. The properties of the reservoir rock surrounding the fault zone are summarized in Table 3 . 
Table 5-2. Permeability Function Parameter Values

\begin{tabular}{lll}
\hline Parameter Name & Units & Value \\
\hline$k_{0}$ & $\mathrm{~m}^{2}$ & $1 \times 10^{-13}$ \\
$c$ & & 10.0 \\
$\sigma$ & $\mathrm{MPa}$ & 45 \\
\hline
\end{tabular}

Table 5-3. Material Properties

\begin{tabular}{llll}
\hline Property Name & Units & Value for Dense Rock & Value for Fault Zone \\
\hline Permeability, $k$ & $\mathrm{~m}^{2}$ & $1 \times 10^{-18}$ & $1 \times 10^{-13}$ \\
Porosity, $\phi$ & -- & $1.0 \times 10^{-2}$ & $1.0 \times 10^{-4}$ \\
Thermal conductivity, $\kappa$ & $\mathrm{W} / \mathrm{m} \mathrm{K}$ & 4.0 & 4.0 \\
Specific heat of water, $c_{p}$ & $\mathrm{~J} / \mathrm{kg} \mathrm{K}$ & 4186 & 4186 \\
Specific heat of rock, $c$ & $\mathrm{~J} / \mathrm{kg} \mathrm{K}$ & 920 & 920 \\
Rock Density, $\rho$ & $\mathrm{kg} / \mathrm{m}^{3}$ & 2500 & 2500 \\
Pore compressibility, $C_{\phi}$ & $\mathrm{Pa}^{-1}$ & $4.0 \times 10^{-10}$ & $1.0 \times 10^{-7}$ \\
Bulk compressibility, $C_{b}$ & $\mathrm{~Pa}^{-1}$ & $4.0 \times 10-^{12}$ & $1.0 \times 10^{-11}$ \\
Bulk modulus, $K$ & $\mathrm{GPa}$ & 250 & 100 \\
Poisson's ratio, $v$ & -- & 0.1 & 0.2 \\
Young's modulus, $E$ & $\mathrm{GPa}$ & 600 & 180 \\
Water density, $\rho_{w}$ & $\mathrm{~kg} / \mathrm{m}^{3}$ & 936.42 & 936.42 \\
Water viscosity, $\mu_{w}$ & $\mathrm{~Pa} \mathrm{~s}$ & $2.0151 \times 10^{-4}$ & $2.0151 \times 10^{-4}$ \\
Water compressibility, $C_{w}$ & $\mathrm{~Pa}-1$ & $4.475 \times 10^{-10}$ & $4.475 \times 10^{-10}$ \\
\hline
\end{tabular}

\subsection{Initial and Boundary Conditions and Sources for Benchmark Problem 1}

The initial conditions for fluid pressure and temperature for the entire domain are assumed to be $20 \mathrm{MPa}$ and $140^{\circ} \mathrm{C}$, respectively.

For a symmetric Cartesian domain, the bottom, left and front sides are treated as no flow and zero heat flux boundaries. For simplicity, the top-side is also treated as no flow and zero heat flux boundary since all fluid flow essentially occurs in the faulted zone and the cooling front only barely propagates into the overburden rock within the test period. The right- and back-sides are treated as fixed fluid pressure and temperature boundaries, with values equal to the initial fluid pressure and temperature in the reservoir. For a cylindrical coordinate system, a Dirichlet (constant pressure) boundary condition is defined at the outer radial boundary surface. All other boundary surfaces are zero-flux type boundaries except for the injection boundary at the well.

Fluid injection was modeled as a constant mass source of water of $80 \mathrm{~kg} / \mathrm{s}$ at the circular well with a radius of $0.15 \mathrm{~m}$. For example, the first inner radial grid cell at $\mathrm{r}(1)=0.15 \mathrm{~m}$ for a $45^{\circ}$ wedge domain assumes $10 \mathrm{~kg} / \mathrm{s}$ injection source rate, i.e., $1 / 8$ of the specified rate of $80 \mathrm{~kg} / \mathrm{s}$ for the full cylinder.

\subsection{Solution Metrics for Benchmark Problem 1}

This problem is based on field observations that demonstrate nonlinear feedback from pressure changes on the permeability field. The basis for comparison was to report a number of pressure and permeability time series at specified points in the fault zone. Table 5-4 summarizes the comparison metrics for this problem. Eight teams submitted results for both the leak-off and no leak-off cases, and three teams 
submitted results for only one case.

Table 5-4. Comparison Metrics for Benchmark Problem 1

\begin{tabular}{lllll}
\hline Metric & Parameter & $\begin{array}{l}\text { Cartesian } \\
\text { Location } \\
(\mathrm{m})\end{array}$ & $\begin{array}{l}\text { Radial } \\
\text { Location } \\
(\mathrm{m})\end{array}$ & $\begin{array}{l}\text { Time } \\
(\mathrm{s})\end{array}$ \\
\hline Time & 1. Pressure & $\mathrm{x}=10$ & $\mathrm{r}=14.142$ & Continuous \\
Series & 2. Permeability & $\mathrm{y}=10$ & & \\
& 3. Water Density & $\mathrm{z}=2,000$ & & \\
4. Water Viscosity & & Along line $\mathrm{x}=\mathrm{y}$ & Line from & $1.0 \times 10^{4}$ \\
& 1. Pressure & $\mathrm{z}=2,000$ & & \\
& 2. Permeability & & $\mathrm{r}=2,828.4$ & \\
& 3. Water Density & & & \\
& 4. Water Viscosity & &
\end{tabular}

\subsection{Results for Benchmark Problem 1}

Benchmark Problem 1 was designed to provide the simulation codes with the least complexity in terms of the coupled processes being modeled, and to have the submitted results reflect the overall findings in this study; where the degree of uncertainty in the collective simulation results was correlated with the number of the modeled coupled processes and their complexity. Note that the structure of the "no-leak-off" scenario allowed for a one-dimensional solution, whereas the "leak-off" scenario required a multidimensional approach. Results are presented by output parameter (i.e., pressure, permeability, aqueous density, and aqueous viscosity) with each figure pair reporting raw data and the ISO-13528 average, standard deviation, and uncertainty. For each output parameter there are two pairs of plots, one versus time at the reference location and one versus distance at the reference time.

Pressure solutions for the "no-leak-off" scenario are shown versus time and distance for 10 of the 11 participating teams in Figs. 5-2 and 5-3, respectively. For the "no-leak-off" scenario the pressure at $14.142 \mathrm{~m}$ rapidly rises in response to the fluid injection and then asymptotically approaches a constant value. The spatial distribution at $10^{4} \mathrm{~s}$, shows a sharp decay in pressure from the centroid, followed by an exponential decay toward the outer boundary pressure. Comparisons of pressure solutions among the participants for the "no-leak-off" scenario are shown versus time and distance in Figs. 5-4 and 5-5, respectively. Uncertainty in the simulation pressures are relatively low, being generally 2.5 orders of magnitude lower than their robust averages, both in the temporal and spatial results. Maximum uncertainties pressure occur closest to the injection well, but are generally not above $0.1 \mathrm{MPa}$. 


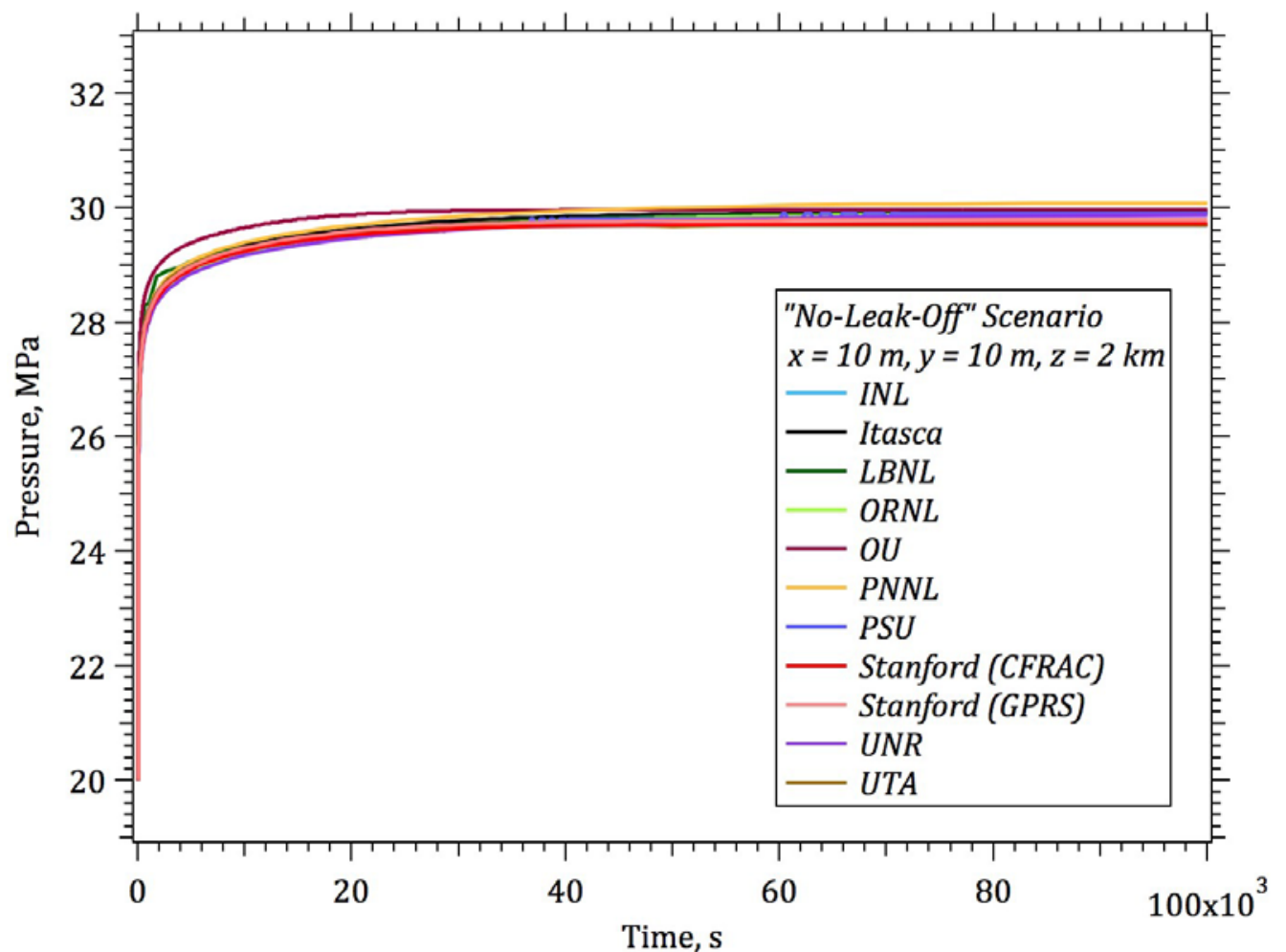

Figure 5-2 Pressure solution versus time at $\mathrm{r}=14.142 \mathrm{~m}$ for the "no-leak-off" scenario for the 10 teams.

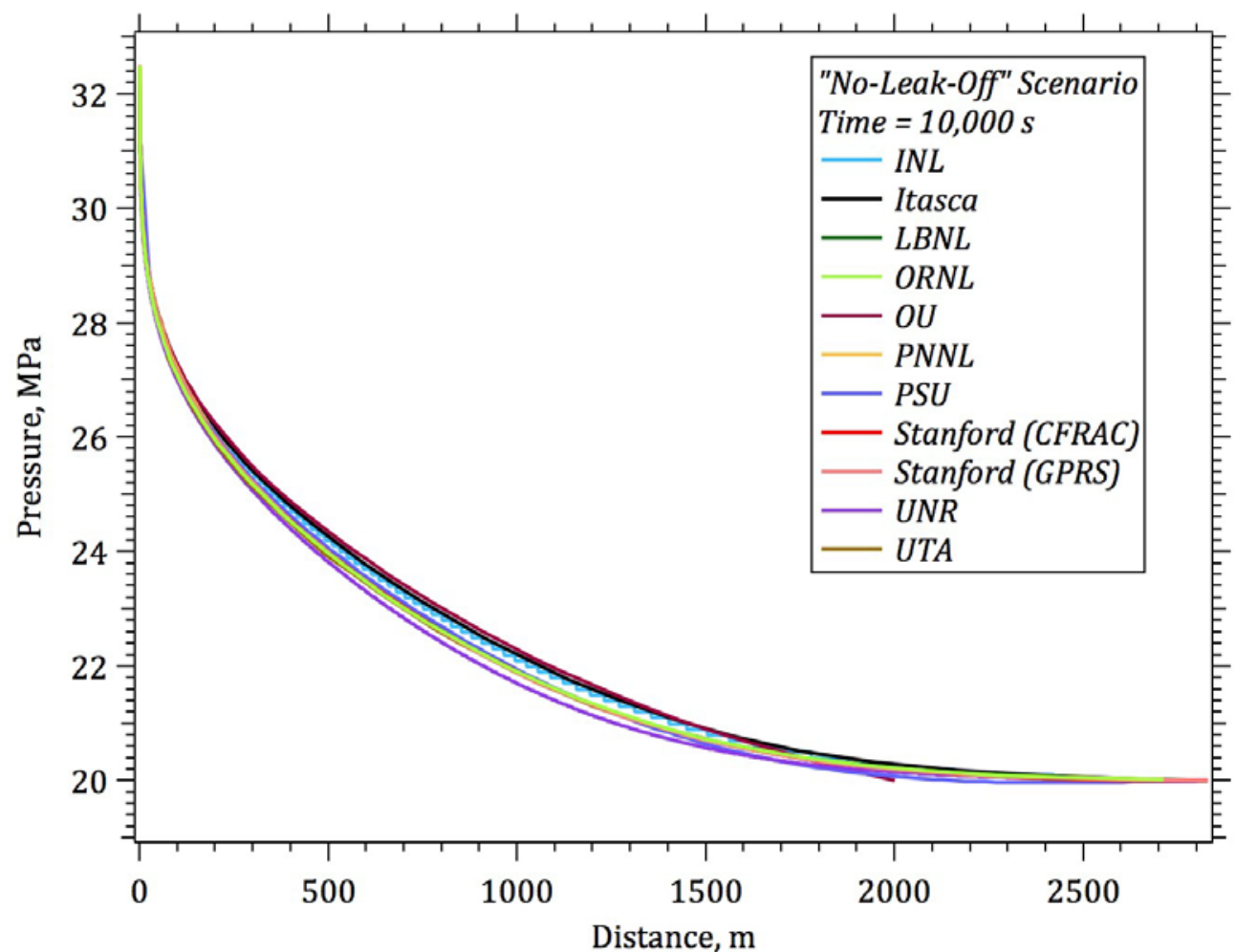

Figure 5-3 Pressure solution versus distance a at $\mathrm{t}=10^{4} \mathrm{~s}$ for the "no-leak-off" scenario for the 10 teams 


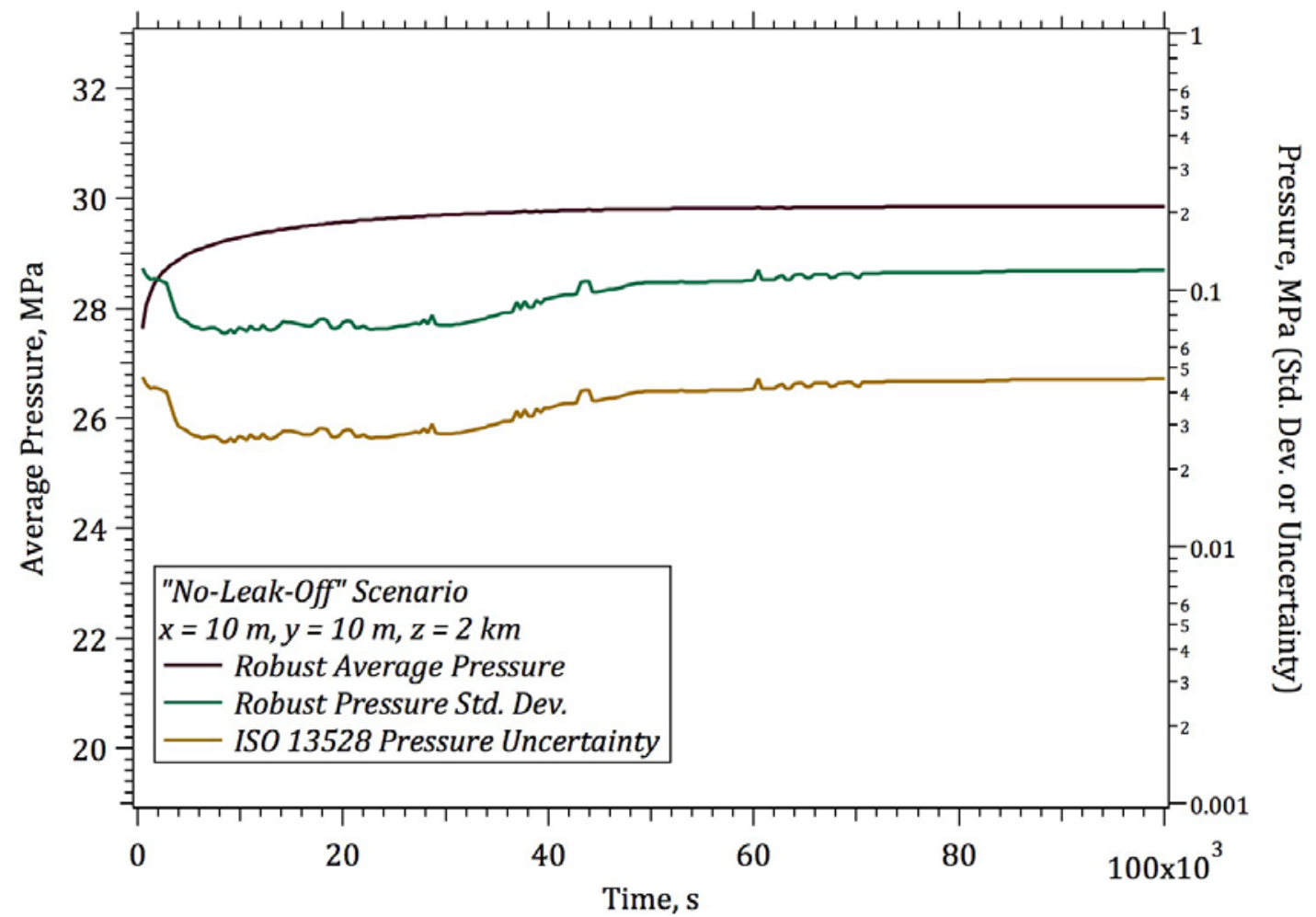

Figure 5-4 Robust average, standard deviation, and ISO 13528 uncertainty in pressure solution versus time at $r=14.142 \mathrm{~m}$ for the "no-leak-off" scenario for the 10 teams

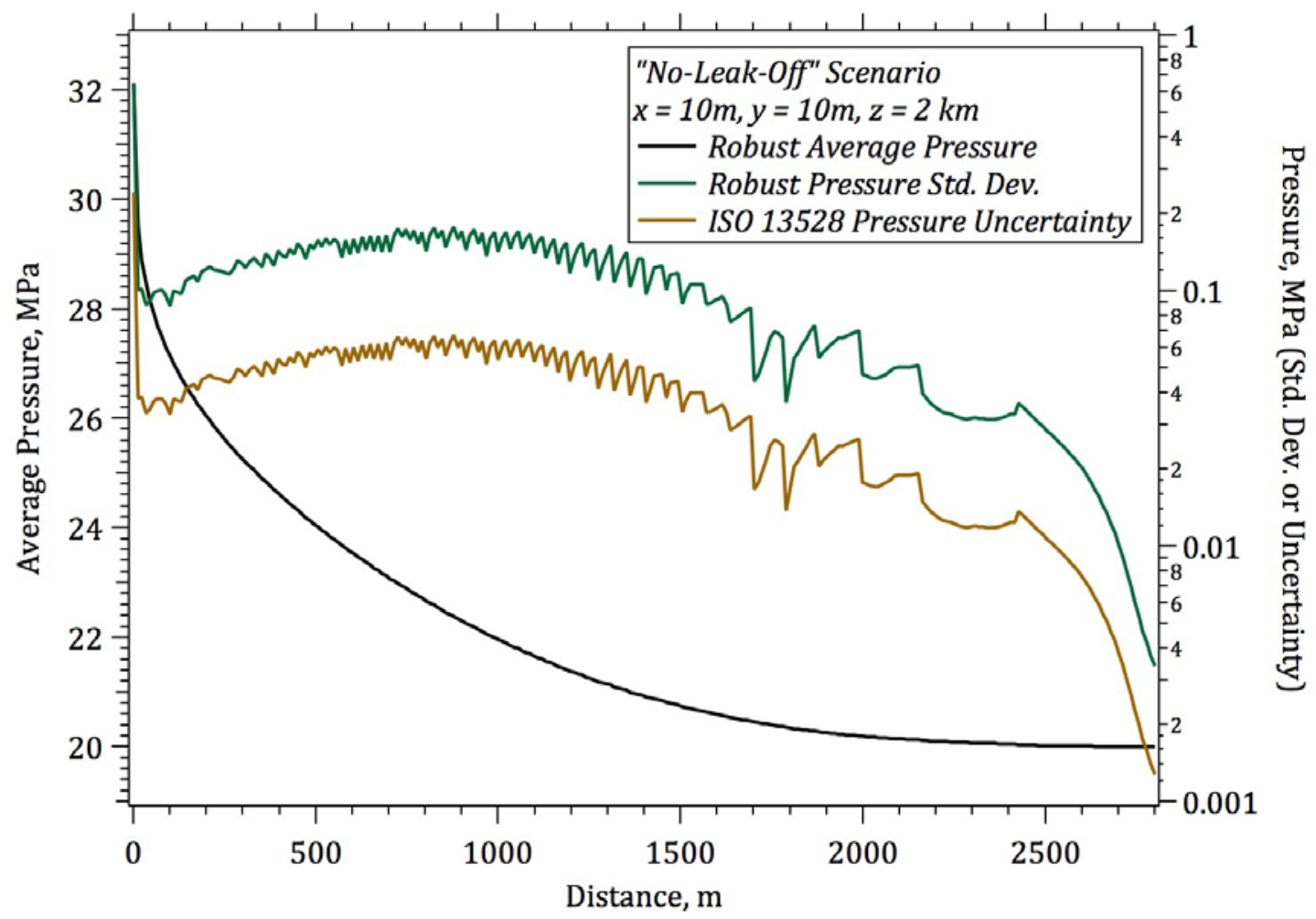

Figure 5-5 Robust average, standard deviation, and ISO 13528 uncertainty in pressure solution versus distance at $\mathrm{t}=10^{4} \mathrm{~s}$ for the "no-leak-off" scenario for the 10 teams 
Permeability solutions for the "no-leak-off" scenario are shown versus time and distance for the 10 participating teams in Figs. 5-6 and 5-7, respectively. As the expression for permeability as a function of pressure was provided, see Eqn. (5.2), the agreement in permeability between the teams is comparable to those for pressure. Comparisons of permeability solutions among the participants for the "no-leak-off" scenario are shown versus time and distance in Figs. 5-8 and 5-9, respectively. Uncertainty in the simulation permeability are relatively low, being generally 1.5 to 2.0 orders of magnitude lower than their robust averages, both in the temporal and spatial results. Maximum uncertainties permeability occur closest to the injection well, but are generally not above $10^{-14} \mathrm{~m}^{2}$, with the robust averages being around $10^{-12} \mathrm{~m}^{2}$ at those points in space and time.

The "leak-off" scenario considered the reservoir rock to have a low, but finite intrinsic permeability (i.e., $10^{-18} \mathrm{~m}^{2}$ ), meaning it could receive water from the fault zone. Pressure solutions for the "leak-off" scenario are shown versus time and distance for all 11 participating teams in Figs. 5-10 and 5-11, respectively. The pressure responses predicted by all the participating teams for the "leak-off" scenario were in good agreement, and as expected were lower than those for the "no-leak-off" scenario. Although the rock surrounding the fault zone had an extremely low permeability, sufficient water migrated from the fracture to alter the pressure response between the "no-leak-off" and "leak-off" scenarios. Comparisons of pressure solutions among the participants for the "no-leak-off" scenario are shown versus time and distance in Figs. 5-12 and 5-13, respectively.

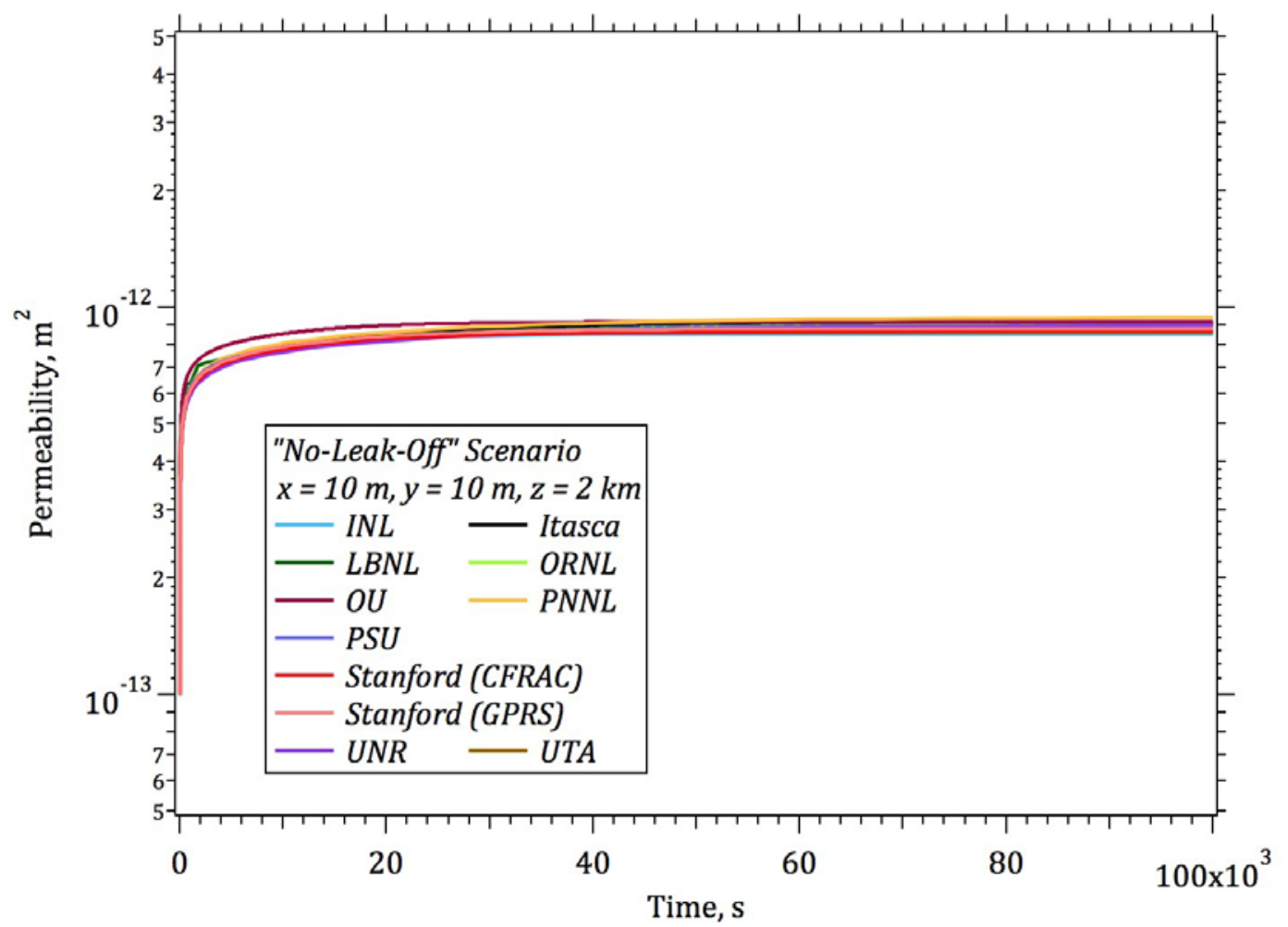

Figure 5-6 Permeability solution versus time at $\mathrm{r}=14.142 \mathrm{~m}$ for the "no-leak-off" scenario for the 10 teams 


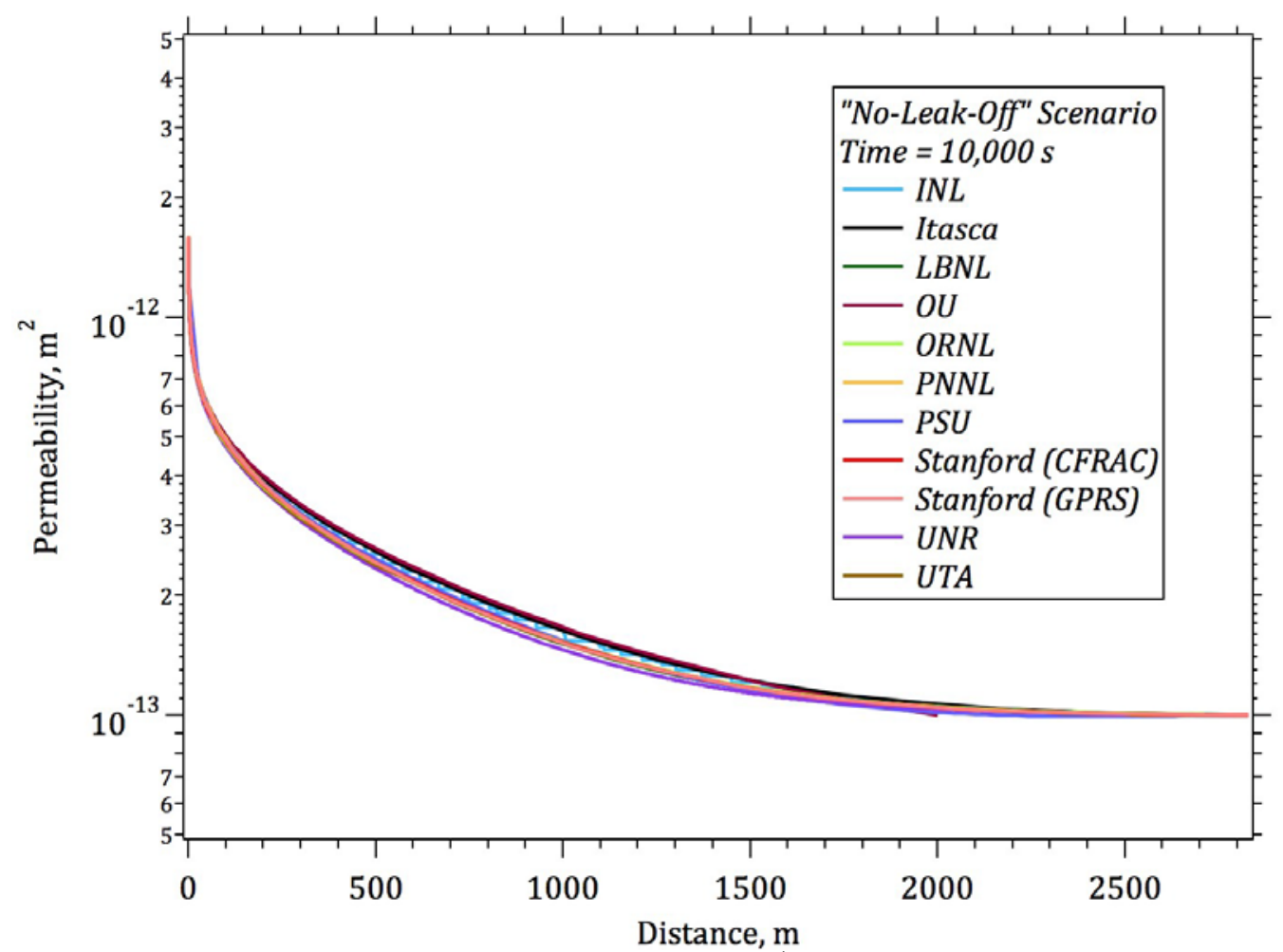

Figure 5-7. Permeability solution versus distance at $\mathrm{t}=10^{4} \mathrm{~s}$ for the "no-leak-off" scenario for the 10 teams

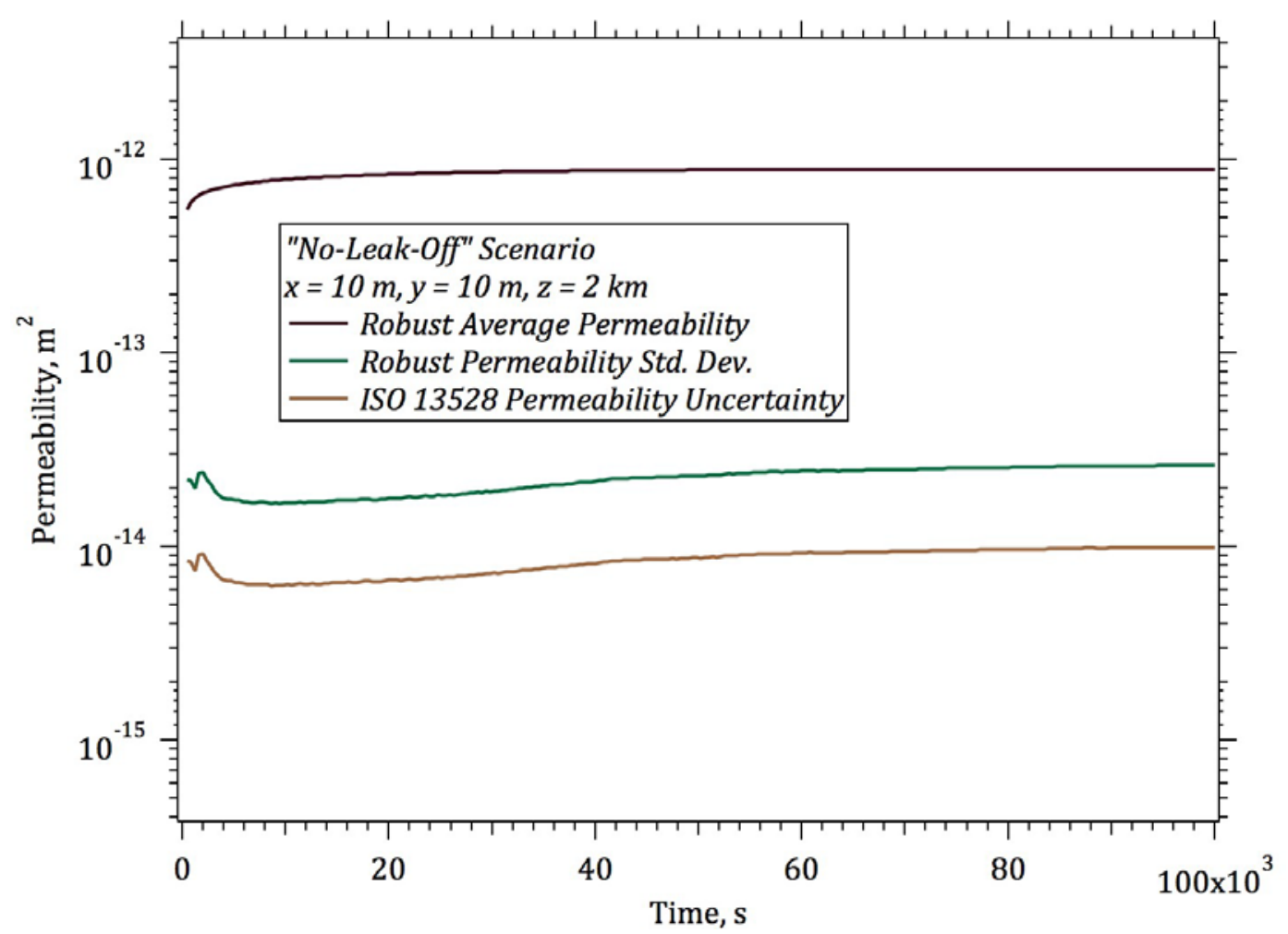

Figure 5-8. Robust average, standard deviation, and ISO 13528 uncertainty in permeability solution versus time at $r=14.142 \mathrm{~m}$ for the "no-leak-off" scenario for the 10 teams 


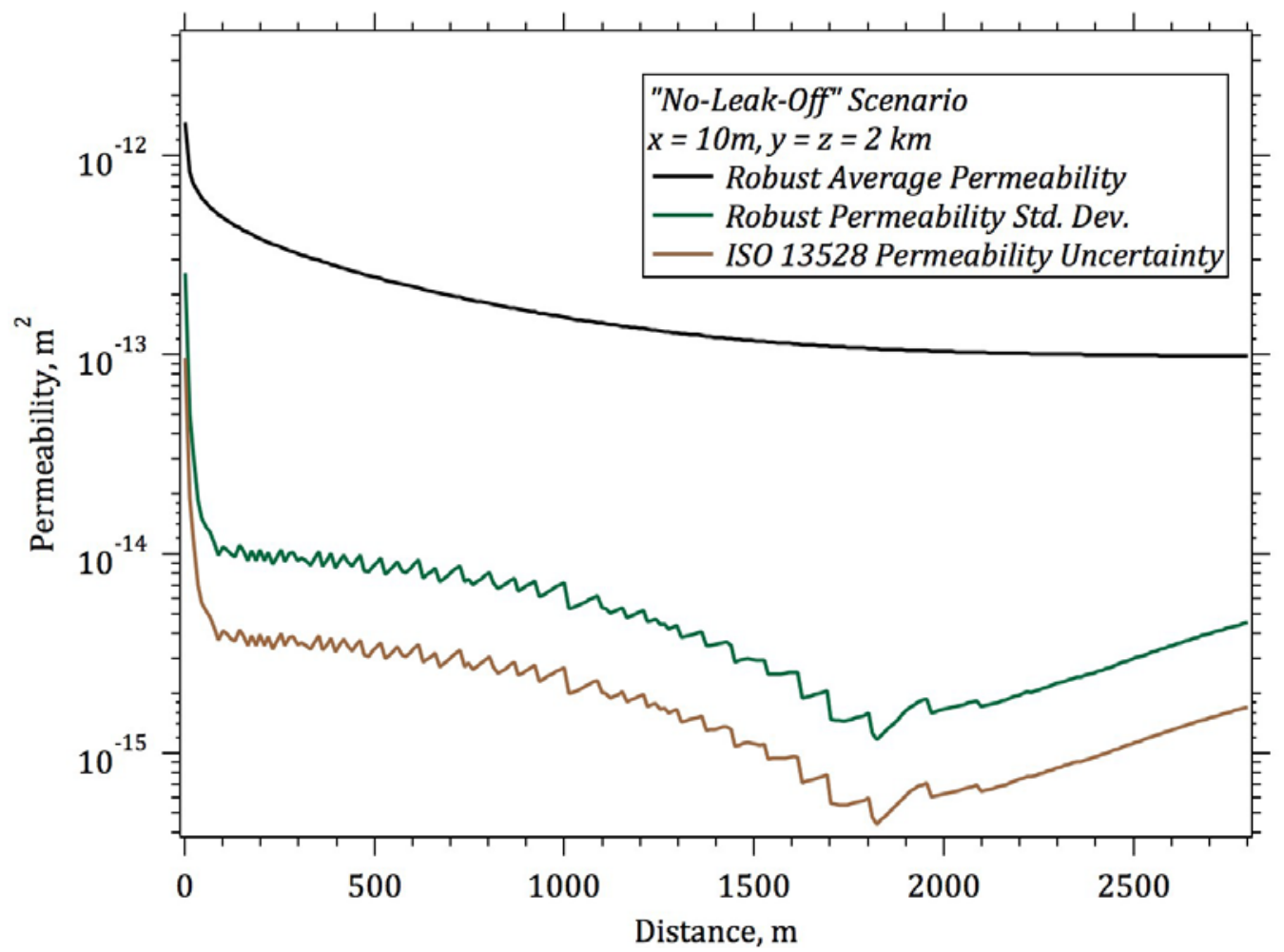

Figure 5-9. Robust average, standard deviation, and ISO 13528 uncertainty in permeability solution versus distance at $\mathrm{t}=10^{4} \mathrm{~s}$ for the "no-leak-off" scenario for the 10 teams

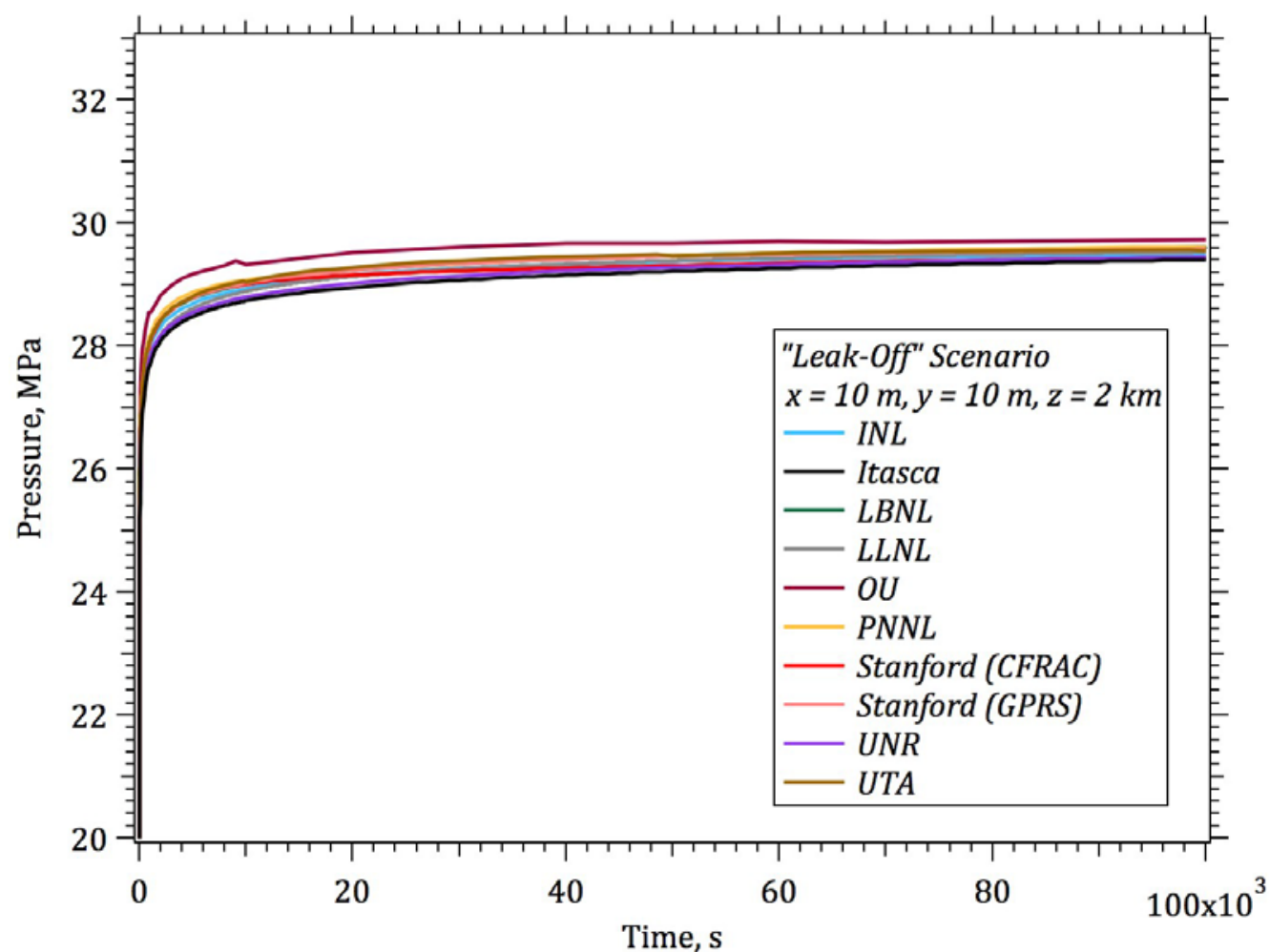

Figure 5-10. Pressure solution versus time at $r=14.142 \mathrm{~m}$ for the "leak-off" scenario for the 11 teams 


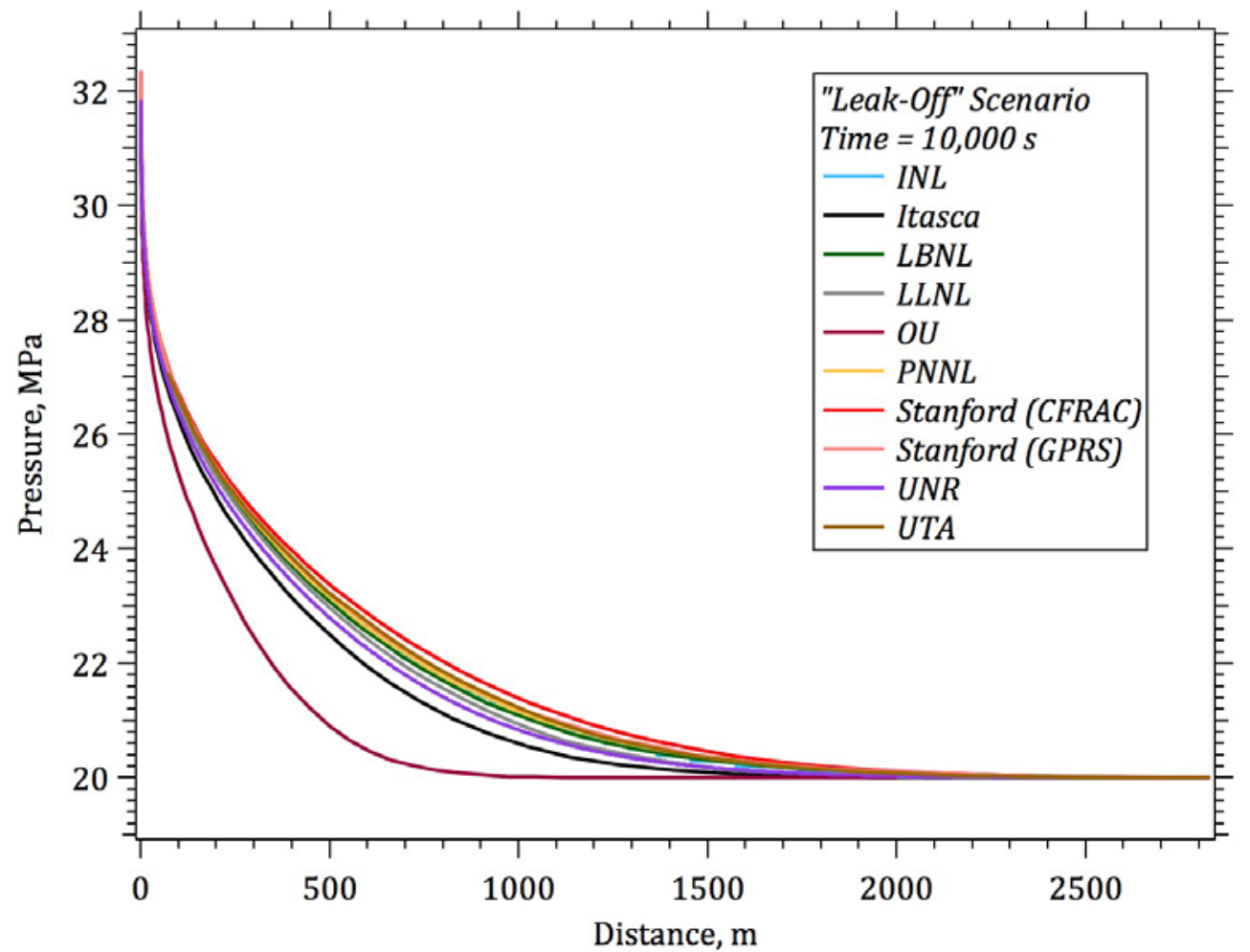

Figure 5-11. Pressure solution versus distance at $\mathrm{t}=10^{4} \mathrm{~s}$ for the "leak-off" scenario for the 11 teams

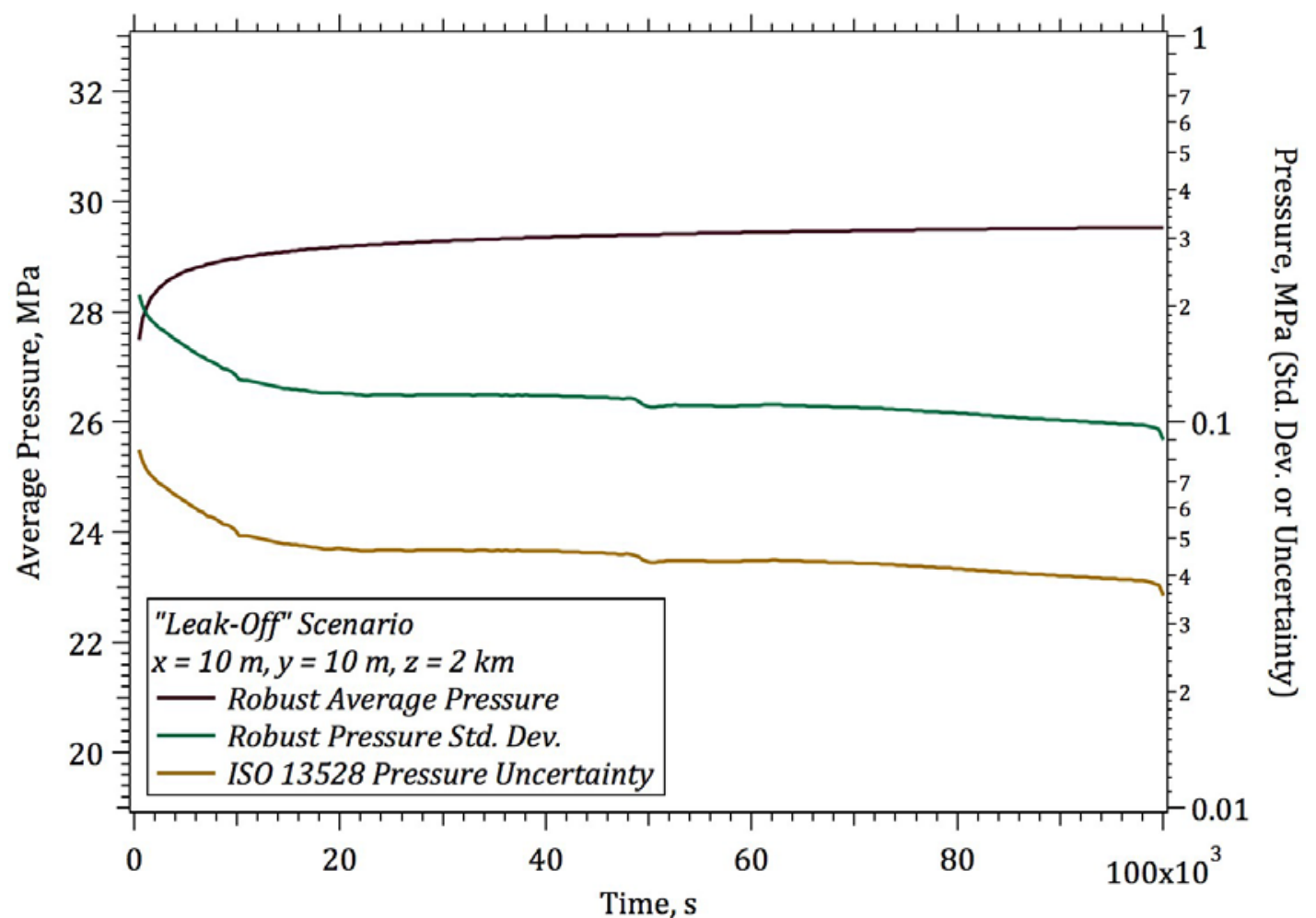

Figure 5-12. Robust average, standard deviation, and ISO 13528 uncertainty in pressure solution versus time at $\mathrm{r}=14.142 \mathrm{~m}$ for the "leak-off" scenario for the 11 teams 


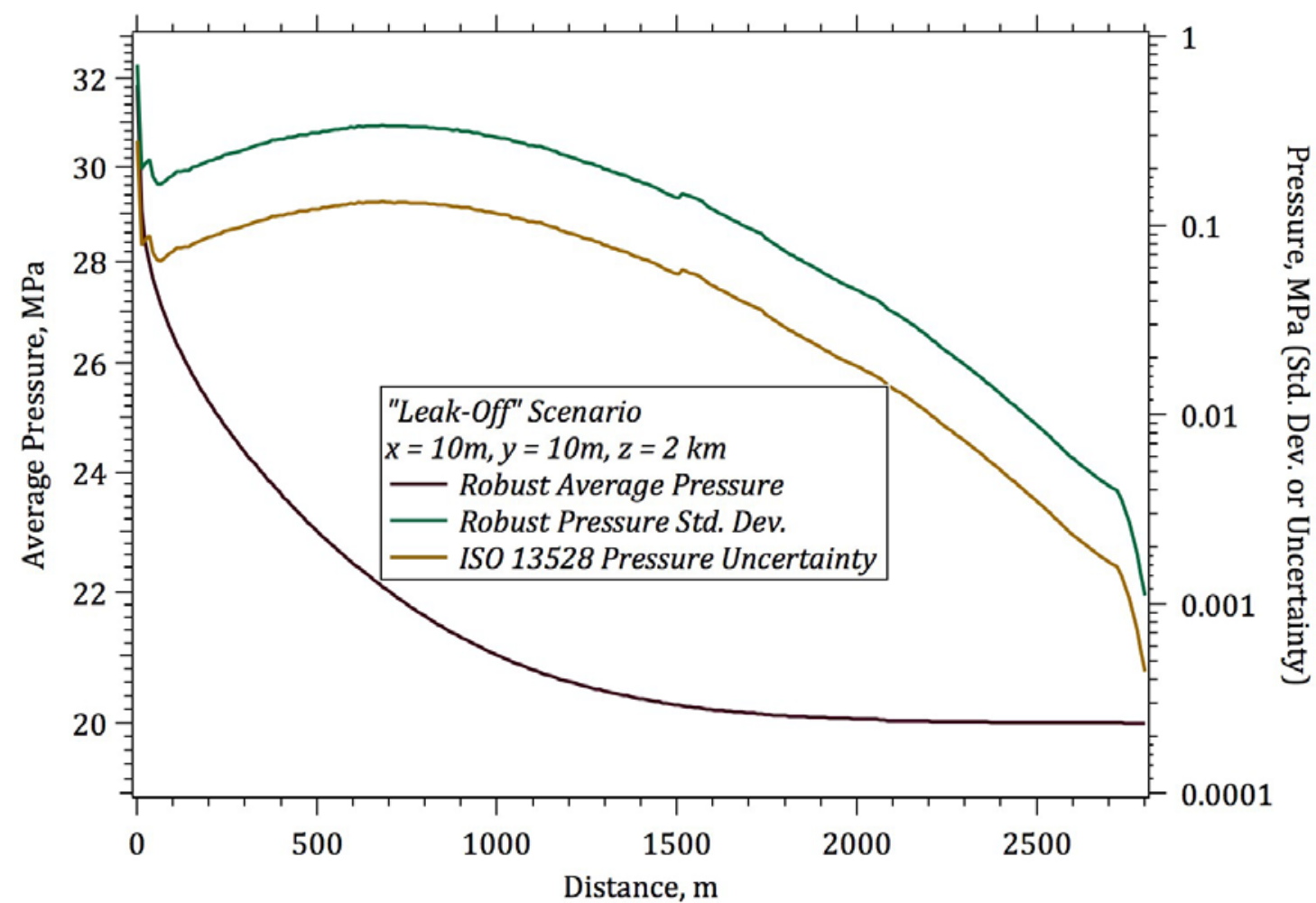

Figure 5-13. Robust average, standard deviation, and ISO 13528 uncertainty in pressure solution versus distance at $\mathrm{t}=10^{4} \mathrm{~s}$ for the "leak-off" scenario for the 11 teams

Uncertainty in the simulation pressures are relatively low, being generally 2.5 orders of magnitude lower than their robust averages, both in the temporal and spatial results. Maximum uncertainties pressure occur closest to the injection well, but are generally not above $0.1 \mathrm{MPa}$.

Permeability solutions for the "leak-off" scenario are shown versus time and distance for the 10 participating teams in Figs. 5-14 and 5-15, respectively. As the expression for permeability as a function of pressure was provided, see Eqn. (5.2), the agreement in permeability between the teams is comparable to those for pressure. Comparisons of permeability solutions among the participants for the "leak-off" scenario are shown versus time and distance in Figs. 5-16 and 5-17, respectively. Uncertainty in the simulation permeability are relatively low, being generally 1.5 to 2.0 orders of magnitude lower than their robust averages, both in the temporal and spatial results. Maximum uncertainties pressure occur closest to the injection well, but are generally not above $10^{-14} \mathrm{~m}^{2}$, with the robust averages being around $10^{-12} \mathrm{~m}^{2}$ at those points in space and time. 


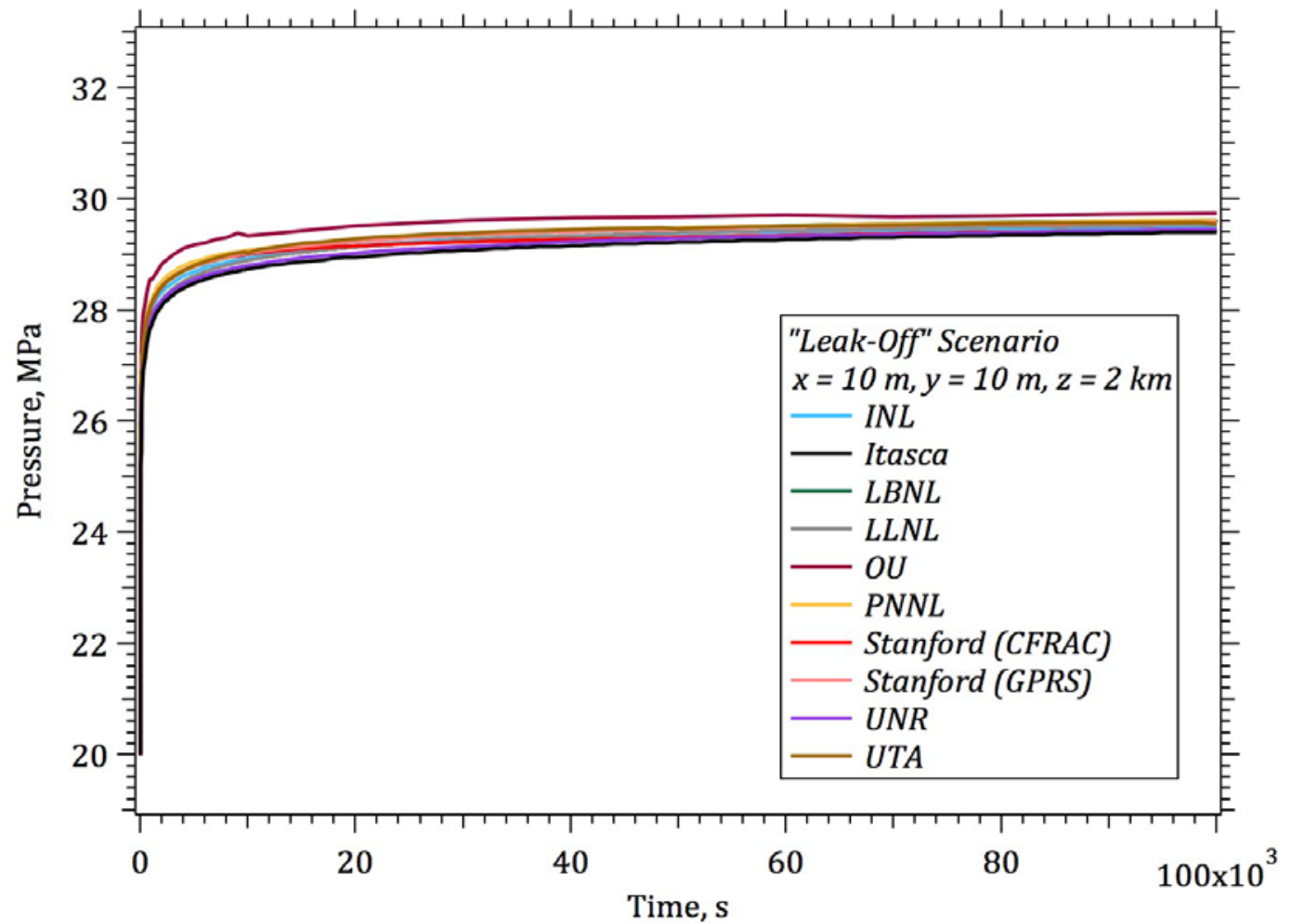

Figure 5-14. Permeability solution versus time at $r=14.142 \mathrm{~m}$ for the "leak-off" scenario for the 10 teams

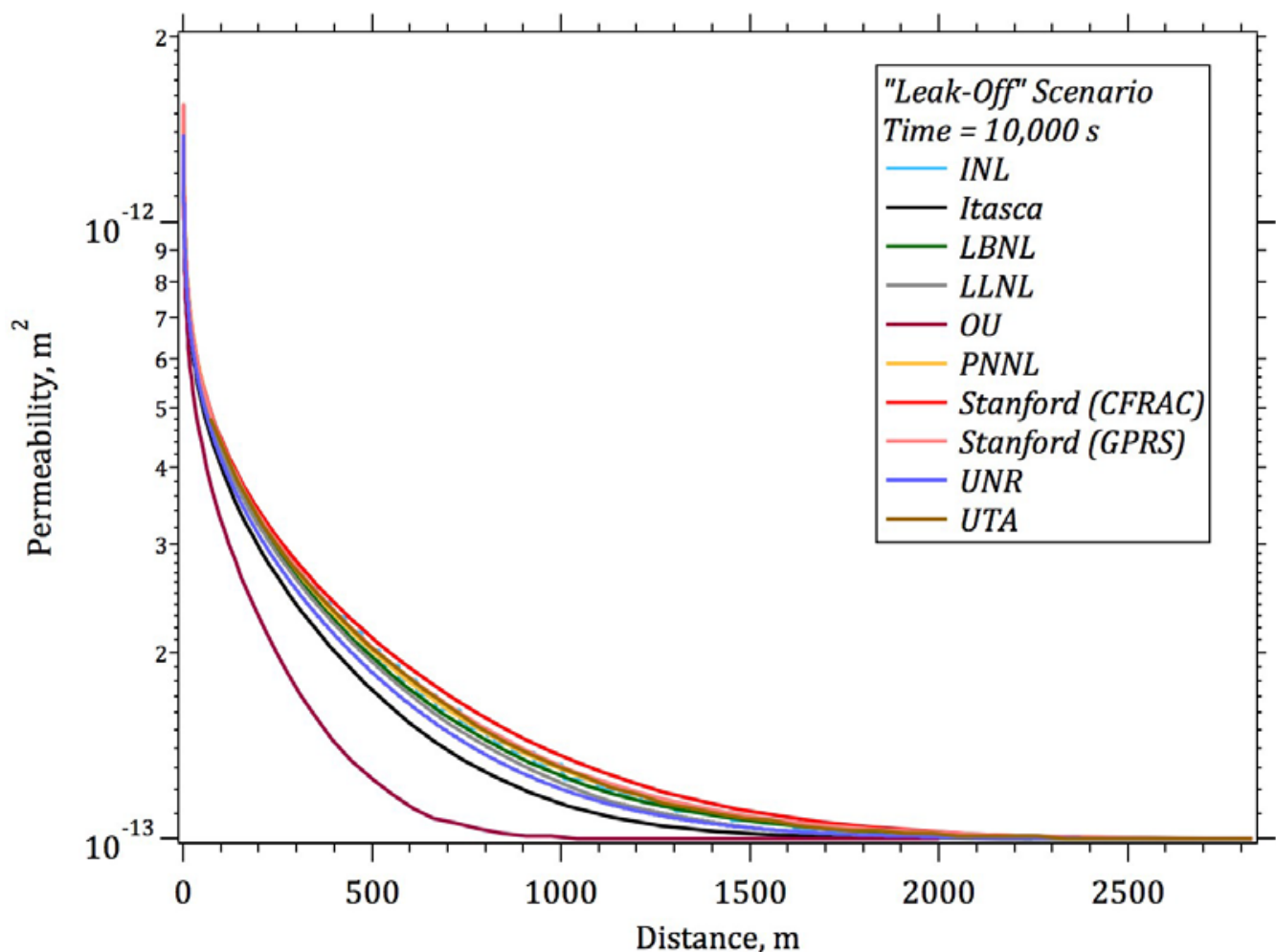

Figure 5-15. Permeability solution versus distance at $\mathrm{t}=10^{4} \mathrm{~s}$ for the "leak-off" scenario for the 10 teams 


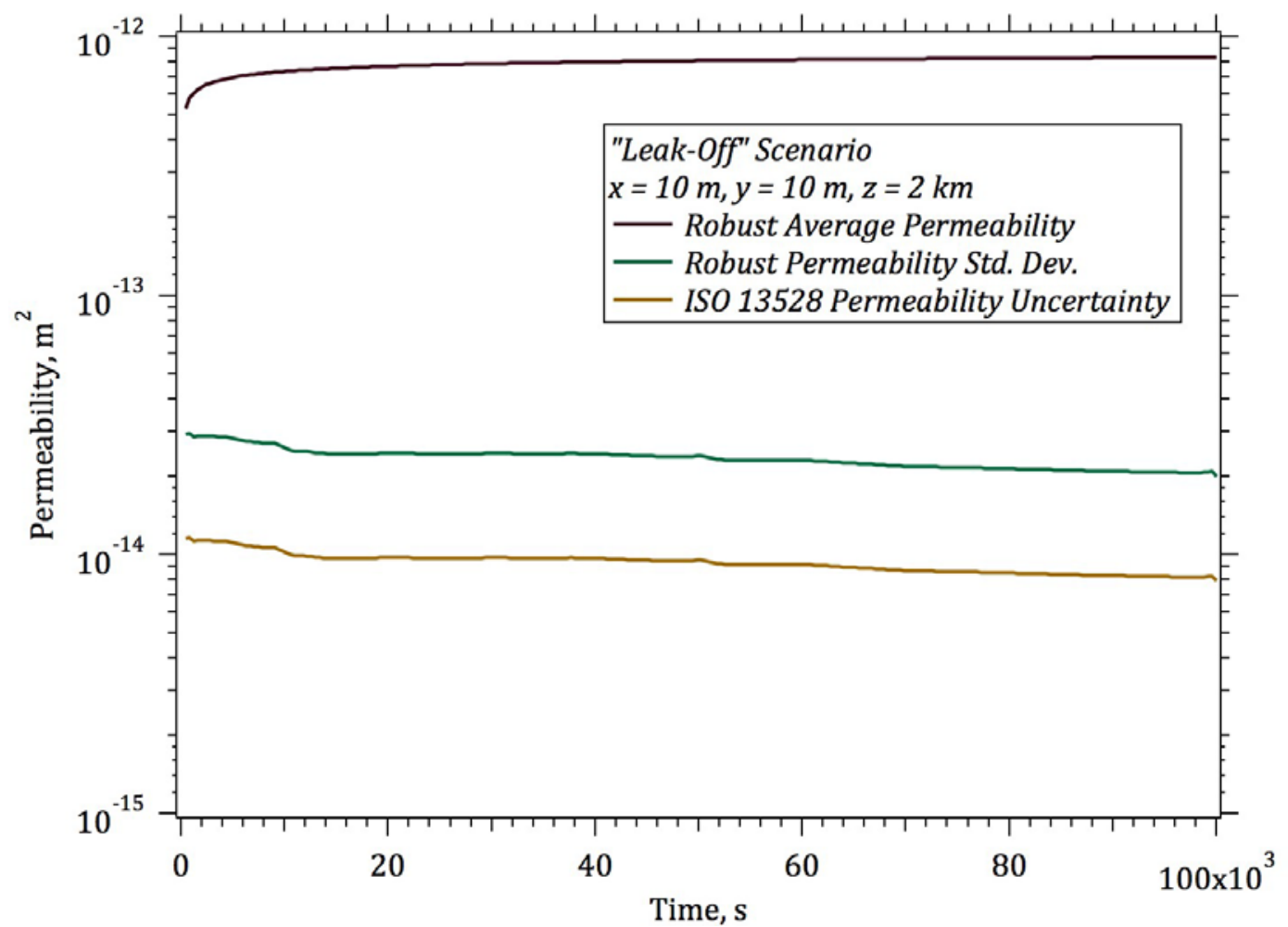

Figure 5-16. Robust average, standard deviation, and ISO 13528 uncertainty in permeability solution versus time at $r=14.142 \mathrm{~m}$ for the "leak-off" scenario for the 10 teams

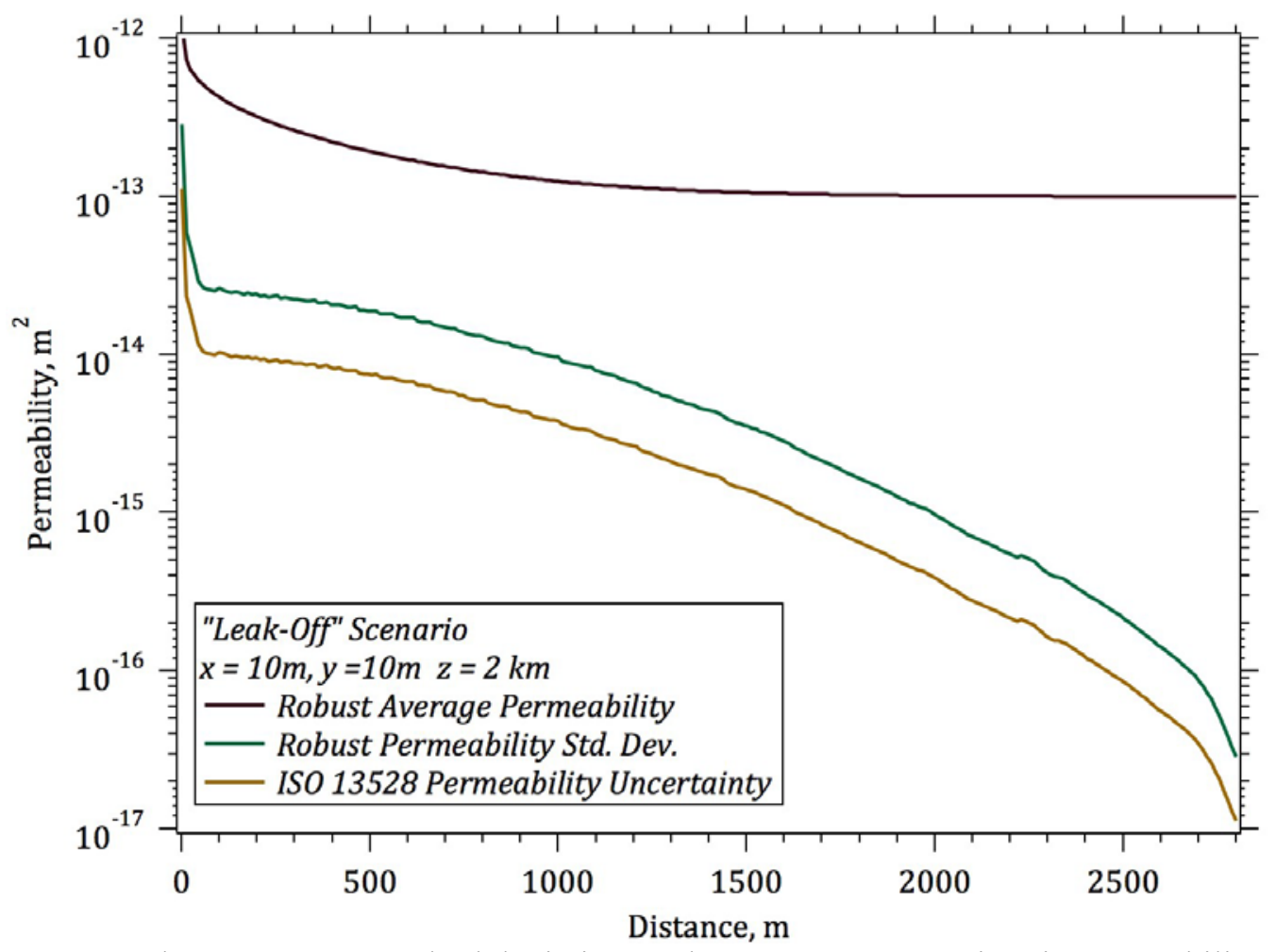

Figure 5-17. Robust average, standard deviation, and ISO 13528 uncertainty in permeability solution versus distance at $\mathrm{t}=10^{4} \mathrm{~s}$ for the "leak-off" scenario for the 10 teams 


\subsection{Discussion of Benchmark Problem 1 Results}

This problem served as an important first test problem for the validation of reservoir simulation codes against each other, and was also used as a means to develop a common dialogue between participants with a reservoir engineering background and those with a hydrogeology background. The assumption of the given poro-elastic characteristics of Nathenson (Nathenson 1999) was followed in all models including the self-propped, open-fracture approach. The codes by all eleven teams delivered qualitatively close, comparable results. Optional outputs for the problem were aqueous density and viscosity. In general most participating teams used equations-of-state for computing water properties that included dependences on pressure and temperature. Some of the teams, however, used constant values for aqueous density and viscosity. As the problem involved isothermal conditions no systematic differences between the various approaches for determining aqueous density and viscosity were noted.

For the time variation of pressure, the computed curves obtained by ten teams using the "no-leak-off" scenario have reached good agreement (see Fig. 5-2), despite the small discrepancy between each other mainly due to the different numerical discretization methods (finite difference method (FDM), finite volume method (FVM), and finite element method (FEM)), geometric topologies (Cartesian and radial), and computational meshes used (triangles, quadrangles, and hexahedron). Similarly, the computed permeability curves also match well (see Fig. 5-6), since the permeability in the fault zone solely depends on the predicted pressure value as shown in Eqn. (5.2). In the "leak-off" scenario, the computed pressure curves match very well (see Fig. 5-10), except for the OU curve whose values appear lower than the others. According to the OU team, the reason is probably that the variable permeability function is also applied to the dense rock zone in OU's simulation, whereas the permeability is assumed to be a constant value in the dense rock zone in all the other teams' simulations. Nevertheless, this does not lead to a huge difference between OU's and the other teams' results in the computed permeability curve (see Fig. 5-14). Moreover, no major difference is observed when the "leak-off" scenario results are compared with those of the "no-leak-off" scenario, mainly because the permeability is five orders of magnitude lower in the dense rock zone than the fault zone.

For spatial variations at the specified time instant, the agreement between different models is also very good. In the "no-leak-off" scenario simulation, the curves match well for both pressure (Fig. 5-3) and permeability (see Fig. 5-7). The OU's results in the "leak-off" scenario simulation differ from the other teams' (see Figs. 5-11 and 5-15), with the reason explained earlier. Overall, the codes by all the eleven participating teams have carried out quite similar prediction of the pressure variation both in time and in space, indicating the high fidelity for simulating the cold-water injection process in the case of no fracture occurrence yet. 


\subsection{Benchmark Problem 2: Shear Stimulation of Randomly Oriented Fractures by Injection of Cold Water Into a Thermo- Poro-Elastic Medium with Stress-Dependent Permeability}

\section{Problem Champion: Sharad Kelkar, Los Alamos National Laboratory}

Benchmark Problem 2 involved injection of cold water at a specified pressure at one location inside a three-dimensional domain with outside boundaries held fixed at initial conditions of pressure, temperature and far-field stresses. This problem was motivated by the shear stimulation treatment of the well 27-15 at Desert Peak, Nevada, in September 2010 (Chabora et al. 2012a) and the subsequent modeling analysis (Dempsey et al. 2013). Observed results of the field stimulation including injection rate, wellhead pressure (WHP) and downhole pressure (DHP) are shown in Fig. 6-1.

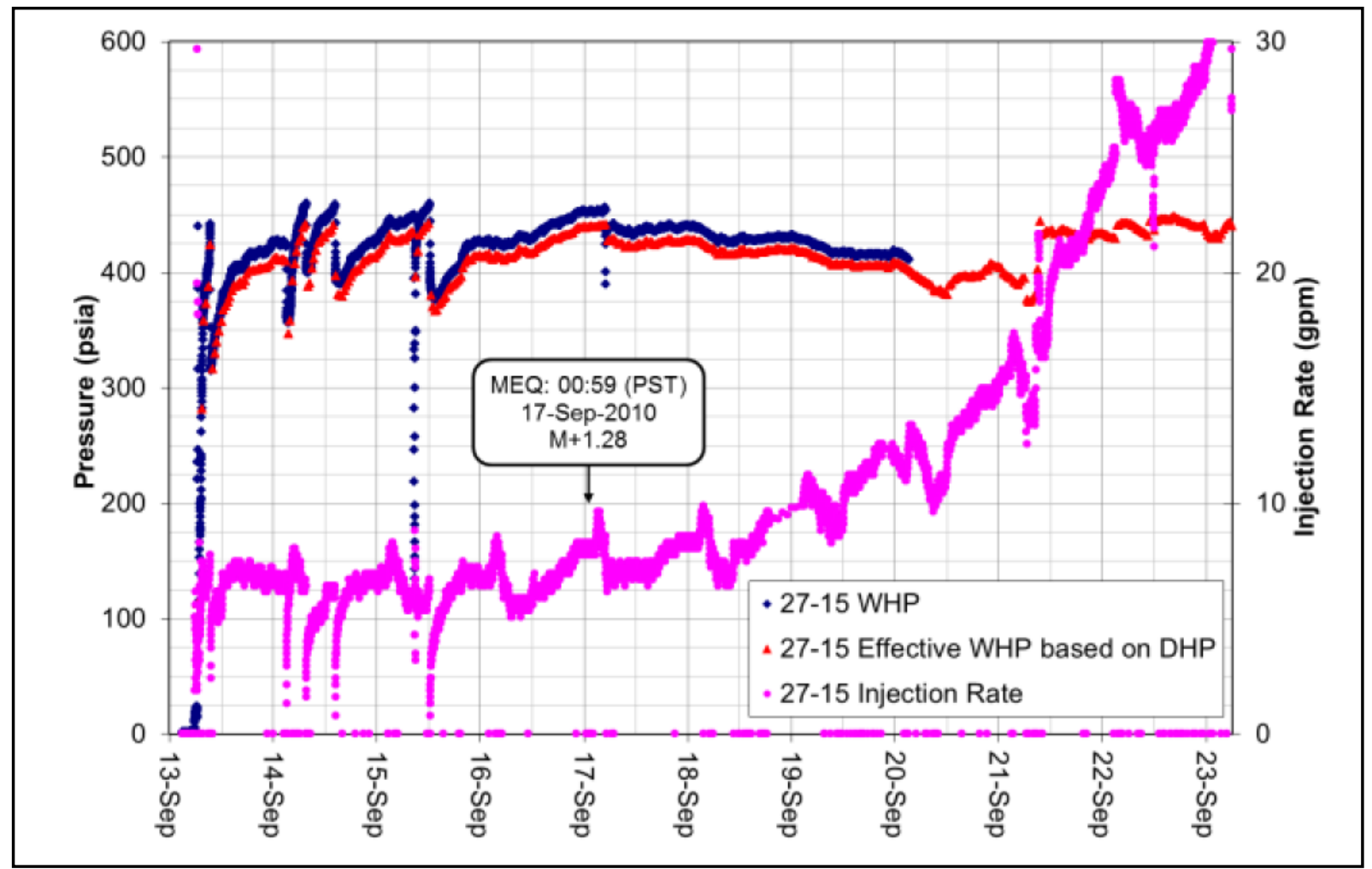

Figure 6-1. Observed results of the field stimulation at Desert Peak well 27-15

The primary objective of the simulations was to predict the injection rate as a function of time. The governing equations for the problem are:

- $\quad$ single-phase fluid mass balance with Darcy's law,

- thermal energy balance including advection and conduction,

- static force balance with linear poro-elasticity (Biot's theory) and thermal stress,

- the Mohr-Coulomb criteria for shear failure using effective stress:

$$
\text { MCStress } \equiv \frac{1}{2}\left(\sigma_{1}-\sigma_{3}\right)\left(\mu^{2}+1\right)^{1 / 2}-\frac{1}{2} \mu\left(\sigma_{1}+\sigma_{3}\right)+\mu P-S_{0}
$$


where $\sigma_{1}$ and $\sigma_{3}$ are the local maximum and minimum principal stresses, $\mathrm{P}$ is the pore pressure, $\mu$ is the coefficient of friction and $S_{0}$ is the cohesion.

- permeability enhancement as a specified function of Mohr-Coulomb stress upon reaching the failure criteria:

$$
\begin{array}{cc}
K=K_{\text {initial }} & \text { if } M \text { CStress }<0 \\
K=K_{\text {initial }}+\left(\frac{M C \text { Stress }}{M C_{\text {ramp }}}\right)\left(K_{\text {max }}-K_{\text {initial }}\right) & \text { if } 0<M C \text { Stress } \leq M C_{\text {ramp }} \\
K=K_{\text {max }} & \text { if } M C \text { Stress }>M C_{\text {ramp }}
\end{array}
$$

Where $K_{\text {initial }}$ is the initial permeability, $K_{\max }$ is the maximum allowed permeability, and $M C_{\text {ramp }}$ is the ramp stress - range of MCStress over which the permeability ramps from the initial to the final value. The simulation duration was 27 days.

Eight teams participated in the solutions using various simplifying assumptions, multiple runs and THM models as listed in Table 6-1. The methods and codes used by each team are described in (White et al. 2015a). Near the injection point, the PNNL, PSU, and LBNL models used a grid spacing of $1 \mathrm{~m}$, the LANL model used a grid spacing of $1.25 \mathrm{~m}$, and the Itasca models used a grid spacing of $5 \mathrm{~m}$. PNNL, PSU and Itasca-local models used a local approximation for calculating the MCStress defined in Eqn. (6.1), not solving the stress-displacement equations and MCStress by using far field stresses and local values of temperature and pressure at each node - these models are referred to as the 'local' models. The PNNL and PSU models solved the three-dimensional porous flow and advective-conductive heat transfer equations. The Itasca-local model solved the porous flow equations while using a simplified semianalytical approach for the temperature calculations. The LBNL and LANL models solved the threedimensional porous flow and advective-conductive heat transfer equations sequentially coupled with the three-dimensional solution of static linear elastic stress-displacement equations. The Itasca-mech model solved the three-dimensional porous flow equations while using a simplified semi-analytical approach for the temperature calculations, and sequentially coupled with the three-dimensional solution of static linear elastic stress-displacement equations. These three models are referred to as the 'non-local' models.

Table 6-1. Participating Teams and Simulators Used in the Solution of Benchmark Problem 2

\begin{tabular}{lll}
\hline Simulation Team & Team Identifier & Code(s) \\
\hline Idaho National Laboratory & INL & FALCON \\
Itasca Consulting Group & Itasca & FLAC $^{3 D}$ \\
Lawrence Berkeley National Laboratory & LBNL & TOUGH and FLAC \\
Los Alamos National Laboratory & LANL & FEHM \\
Pennsylvania State University & PSU & FLAC3D and TOUGHREACT \\
Pacific Northwest National Laboratory & PNNL & STOMP \\
The University of Oklahoma & OU & GEOFRAC \\
The University of Texas at Austin & UTA & CFRAC_UT \\
\hline
\end{tabular}

\subsection{Geometry and Input Data for Benchmark Problem 2}

The injection point was chosen to be one corner of the model and the far field boundaries were placed at 2 $\mathrm{km}$ in the $\mathrm{x}, \mathrm{y}, \mathrm{z}$ directions from the injection point (Fig. 6-2). The problem was taken to have spherical 
symmetry, and only one eighth of the domain was included in the simulation. The material properties are specified in Table 6-2.

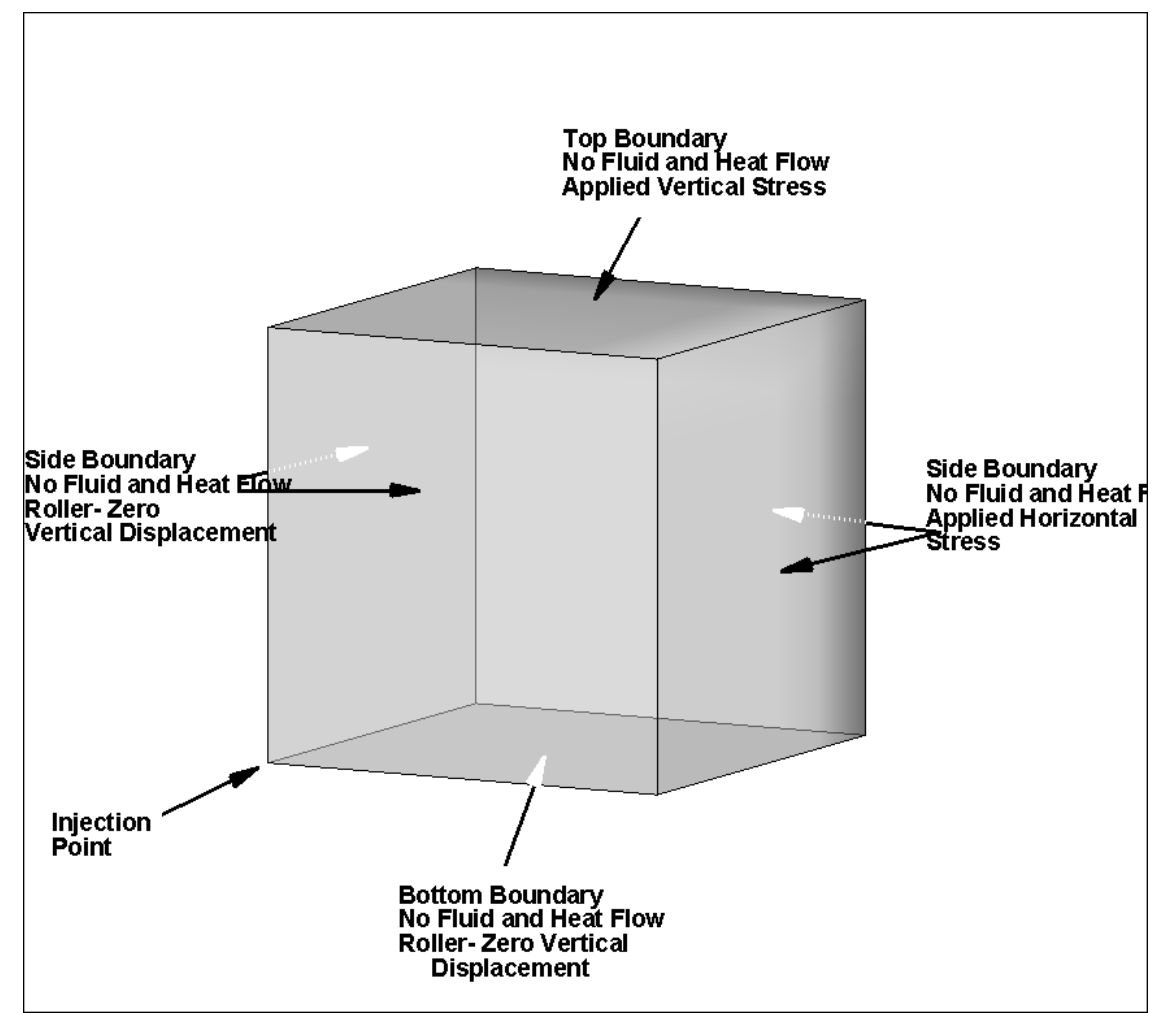

Figure 6-2. Model domain showing the location of the injection point

Table 6-2. Material Property Table for Benchmark Problem 2

\begin{tabular}{lll}
\hline Property Name & Units & Value \\
\hline Initial Permeability, $k_{0}$ & $\mathrm{~m}^{2}$ & $2.6 \times 10^{-15}$ \\
Maximum Permeability, $k$ & $\mathrm{~m}^{2}$ & $55^{*} 2.6 \times 10^{-15}$ \\
Porosity, $\phi$ & -- & 0.1 \\
Thermal Conductivity, $\kappa$ & $\mathrm{W} / \mathrm{m} \mathrm{K}$ & 2.2 \\
Rock Density, $\rho$ & $\mathrm{kg} / \mathrm{m}^{3}$ & 2480 \\
Specific Heat Capacity, $c$ & $\mathrm{~J} / \mathrm{kg} \mathrm{K}$ & 1200 \\
Ramp Stress, $M C_{\text {ramp }}$ & $\mathrm{MPa}$ & 15 \\
Young's Modulus, $E$ & $\mathrm{GPa}$ & 25 \\
Poisson's Ratio, $v$ & -- & 0.2 \\
Coefficient of Thermal Expansion, $\alpha$ & $1 / \mathrm{K}$ & $0.5 \times 10^{-5}$ \\
Biot's Coefficient, $\beta$ & -- & 0.5 \\
Cohesion, $S_{0}$ & $\mathrm{MPa}$ & 3.0 \\
Static Friction Coefficient, $\mu$ & -- & 0.5 \\
\hline
\end{tabular}

\subsection{Boundary and Initial Conditions and Sources for Benchmark Problem 2}

The domain is assumed to be fully liquid water saturated. The initial pore pressure is $9.81 \mathrm{MPa}$ and the reservoir temperature is $190^{\circ} \mathrm{C}$. There is no heat flow or fluid mass flux across the external boundaries. 
The mechanical boundary conditions are rollers on the three coordinate planes going through the injection point (located at $\mathrm{x}, \mathrm{y}, \mathrm{z}=0,0,0$ (i.e. the nodes on the plane normal to the $\mathrm{x}$-axis with coordinates $(0, \mathrm{y}, \mathrm{z})$ with displacement specified as 0 in the X-direction; etc.). A normal stress was applied on the far field model faces, with a downward vertical stress of $22.7 \mathrm{MPa}$ assigned to the top boundary, a minimum principal stress of $13.88 \mathrm{MPa}$ assigned to the far x-direction boundary, and a median principal stress of $18.3 \mathrm{MPa}$ assigned to the far $\mathrm{y}$-direction boundary.

A constant pressure source of liquid water is applied at the node located at $x, y, z=0,0,0$. The control volume associated with this node is $5 \mathrm{~m} \times 5 \mathrm{~m} \times 5 \mathrm{~m}$. The injection pressure is $12.91 \mathrm{MPa}$ and the injection temperature is $100^{\circ} \mathrm{C}$.

\subsection{Solution Metrics for Benchmark Problem 2}

The primary metric for comparing results from different models is the fluid injection rate over time. In addition, temperature, pressure, stress, and displacement versus time were to be reported at the location $\mathrm{x}$ $=\mathrm{y}=\mathrm{z}=2.5 \mathrm{~m}$ and $\mathrm{x}=\mathrm{y}=\mathrm{z}=7.5 \mathrm{~m}$. These locations correspond to a radial distance of $4.33 \mathrm{~m}$ and 12.99 $\mathrm{m}$ respectively.

\subsection{Results for Benchmark Problem 2}

The injection flow rates, scaled by a factor of 8 for the entire spherical domain, as a function of time for the various models are plotted in Fig. 6-3. Also shown in this figure are the two bounding curves calculated using the LANL model shown by dashed grey lines for constant permeabilities with the high $(143 \mathrm{mD})$ and low $(2.6 \mathrm{mD})$ values - these can be expected to be the high and low bounds on the flow rates. In the case of constant formation permeability, the injection rate at a constant injection pressure is seen to decrease with time as expected. However, in this problem the permeability is a function of the MCstress (Mohr-Coulomb stress, Eqn. 6.1), and increases with time due to pore pressure and temperature changes as demonstrated in Fig. 6-4. This causes the flow rate to increase with time. The increase is rapid at the start and slows down as time progresses as can be seen in Fig. 6-3. The predicted injection rates after 25 days fall in the interval of 3.6 to $5.8 \mathrm{~kg} / \mathrm{s}$, with the PSU model predicting the lowest and the LBNL predicting the highest values. The ISO 13528 uncertainty in the injection rate is seen to be $\sim 0.4$ $\mathrm{kg} / \mathrm{s}$, about $8-10 \%$ of the average injection rate (Fig. 6-5). 


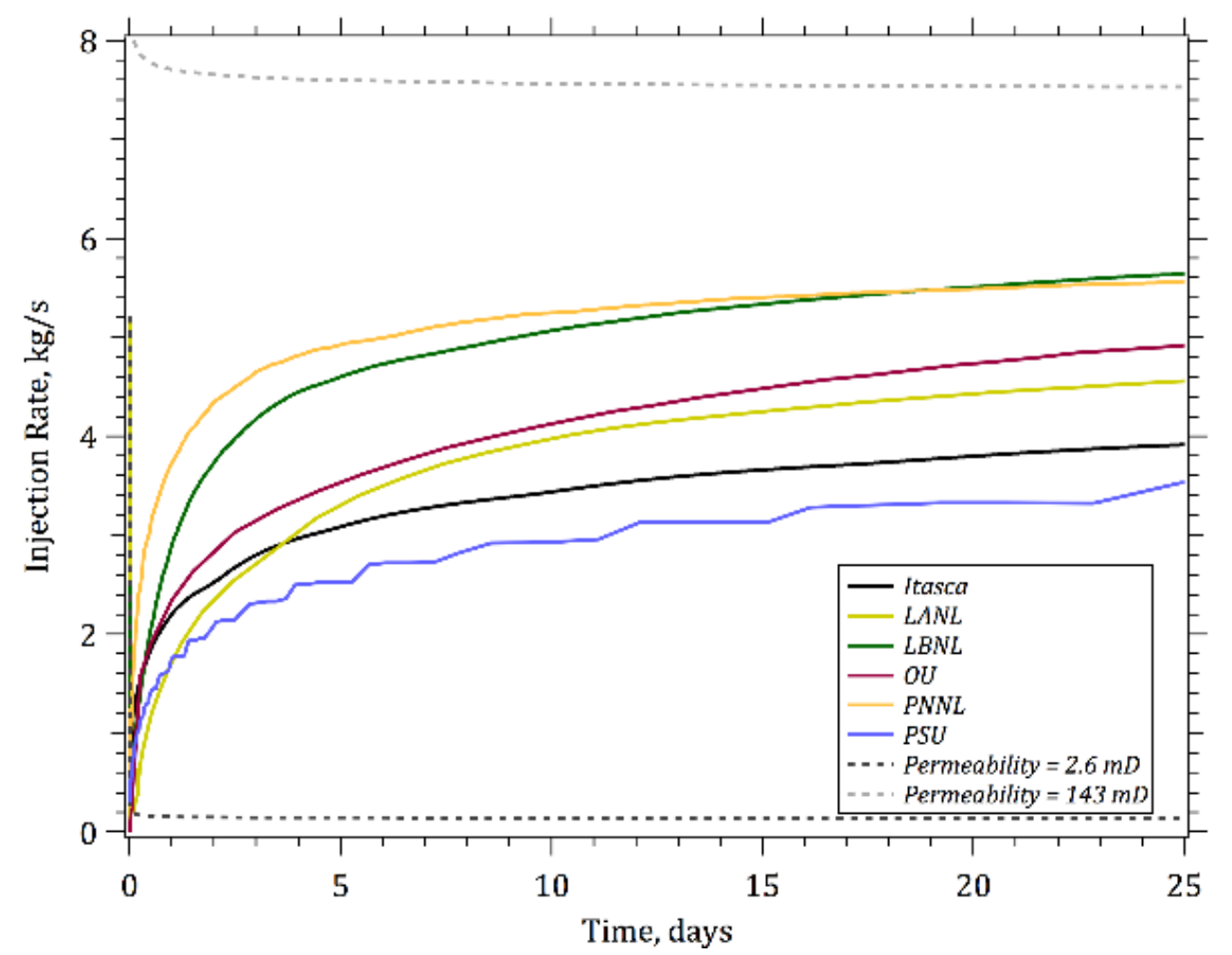

Figure 6-3. Simulation results showing injection rate versus time

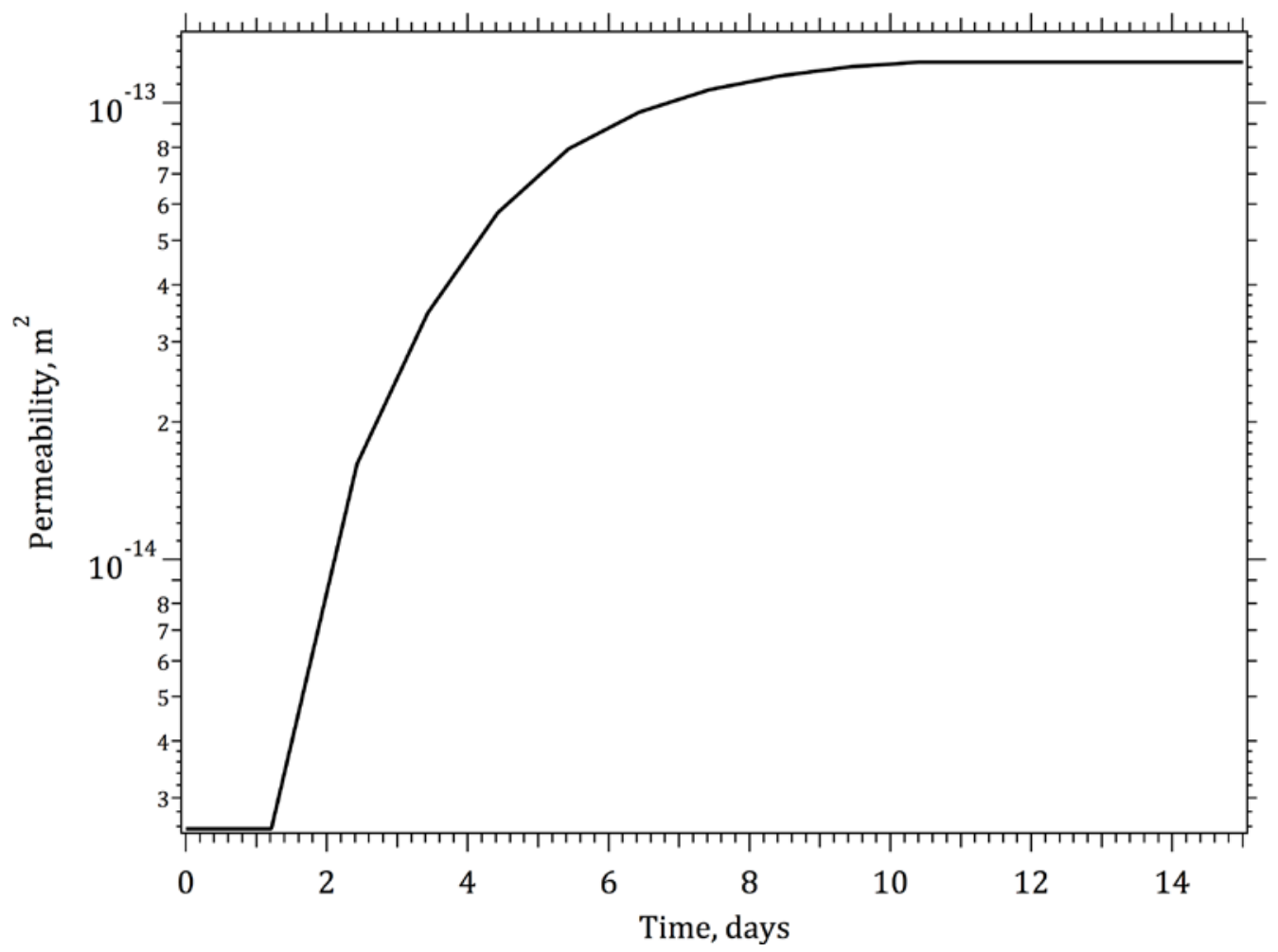

Figure 6-4. Permeability calculated by the LANL model at $4.33 \mathrm{~m}$ from the injection point as a function of time. 


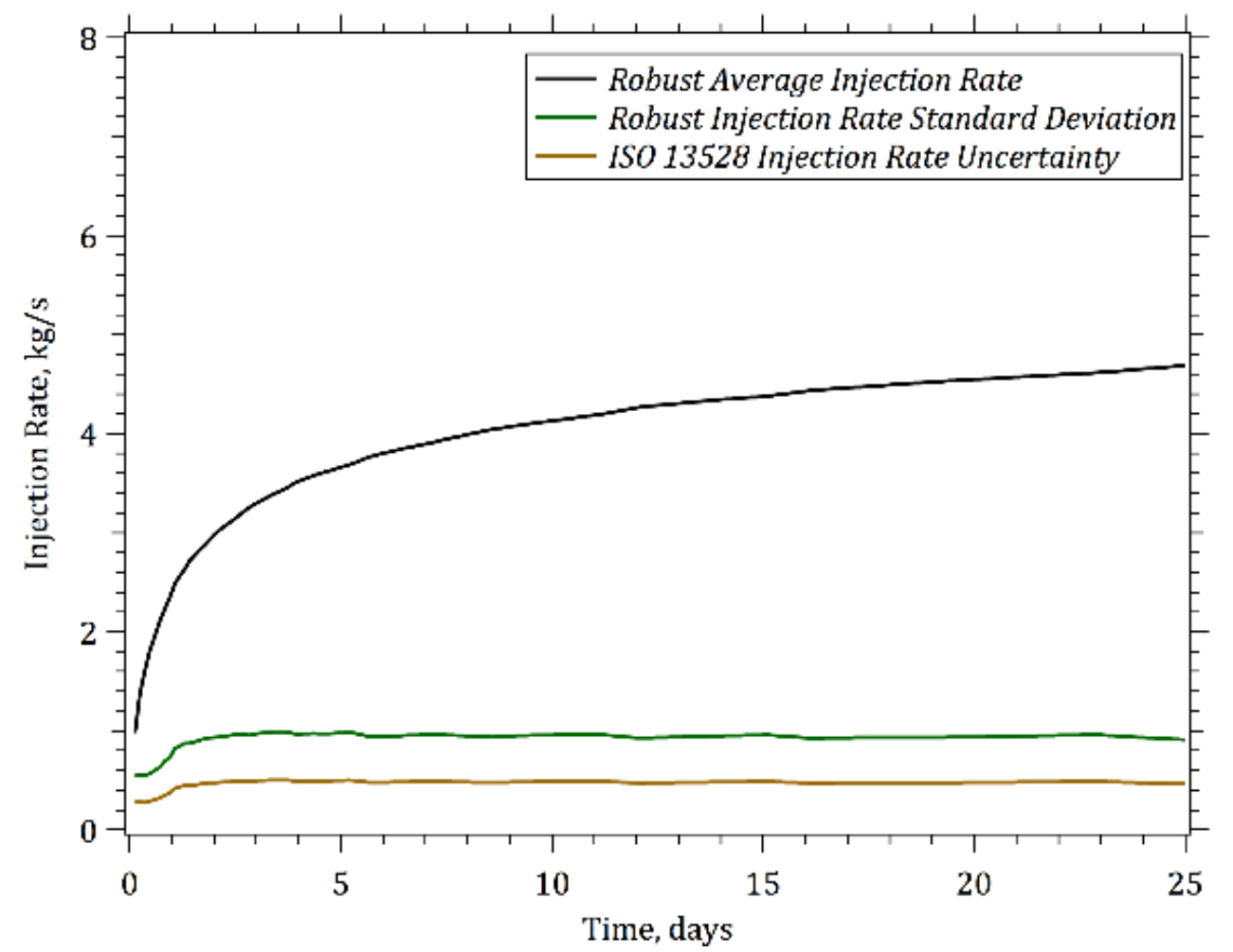

Figure 6-5. ISO 13528 uncertainty in injection rate versus time

Temperature as a function of time at a point $\mathrm{x}=\mathrm{y}=\mathrm{z}=2.5 \mathrm{~m}$ from the injection point is shown in Fig. 6-6. The general trend in Fig. 6-6 is similar for all models, with the temperature dropping from the initial value of $190^{\circ} \mathrm{C}$ to $100^{\circ} \mathrm{C}$, a value close to the injection value, in less than 9 days. Also shown in Fig. 6-6 are the two bounding curves with high $(143 \mathrm{mD})$ and low $(2.6 \mathrm{mD})$ values of permeability. The model curves fall between these two bounding curves as expected, except for the Itasca model, which shows a more rapid decline. The LBNL and OU curves showed a similar temperature decrease, followed by PNNL and then PSU, with LANL having the slowest decrease.

It is interesting to note by comparing Figs. 6-3 and 6-6 that while the PNNL and LBNL models show similar flow rate trends, the LBNL model predicts faster cooling. On the other hand, the OU model predicts lower injection rates than the LBNL model, yet they predict similar temperature declines. The PSU model predicts lower flow rates than the above models, and also shows slower cooling. However, while the LANL model calculates flow rates that are higher than the PSU model, the cooling in the LANL model is slower. The flow rates for the PNNL and PSU models are different as seen in Fig. 6-3, however they predict similar cooling curves. These differences are likely due to the different approaches taken by each model. 


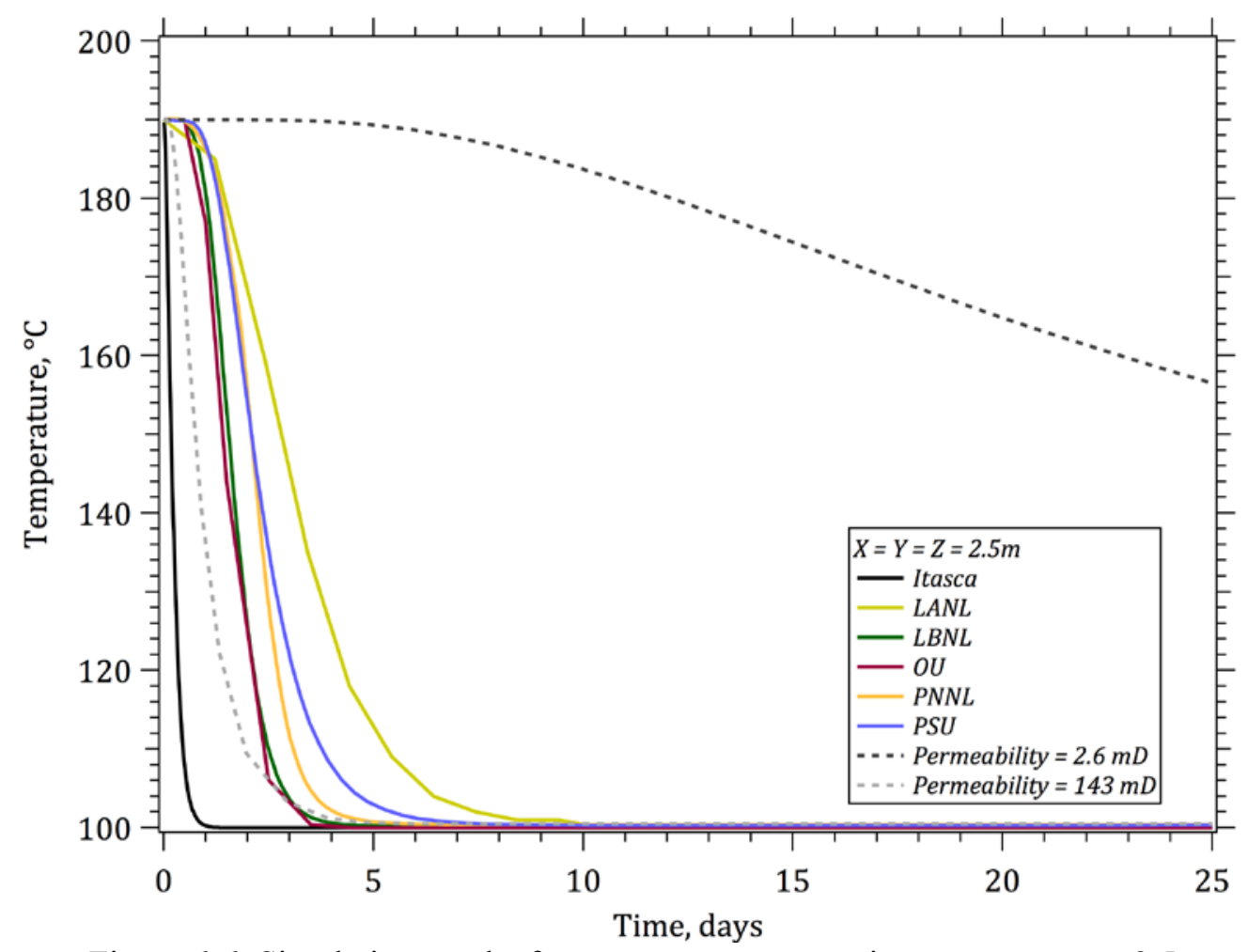

Figure 6-6. Simulation results for temperature versus time at $\mathrm{x}=\mathrm{y}=\mathrm{z}=2.5 \mathrm{~m}$

Temperatures at a point $\mathrm{x}=\mathrm{y}=\mathrm{z}=7.5 \mathrm{~m}$ from the injection point are shown in Fig. 6-7. The general trends are similar, with cooling commencing at a later time due to the increased distance from the injection point. With the exception of the Itasca and OU models, the other model curves fall between the two bounding curves for the high $(143 \mathrm{mD})$ and low $(2.6 \mathrm{mD})$ values of permeability. The relative order of the rate of cooling for LBNL, PSU, and LANL are similar to those in Fig. 6-6, with LBNL showing the most, and LANL showing the least cooling. The PNNL model shows the least amount of cooling at this location.

It is interesting to compare Fig. 6-6 showing the temperature profile at $\mathrm{x}=\mathrm{y}=\mathrm{z}=2.5 \mathrm{~m}$ with that in Fig. $6-7$ at $\mathrm{x}=\mathrm{y}=\mathrm{z}=7.5 \mathrm{~m}$. At $2.5 \mathrm{~m}$, the LANL model showed the slowest cooling, while at $7.5 \mathrm{~m}$, the PNNL model shows the slowest cooling. Also, while the cooling curves for OU and LBNL are close to each other at 2.5 $\mathrm{m}$, they are quite distinct at $7.5 \mathrm{~m}$. The ISO 13528 uncertainty is shown for both locations in Figs. 6-8 an 6-9. The standard deviation and uncertainty in the temperature is greater in early time at $\mathrm{z}=2.5 \mathrm{~m}$ and at later time at $\mathrm{z}=7.5 \mathrm{~m}$. 


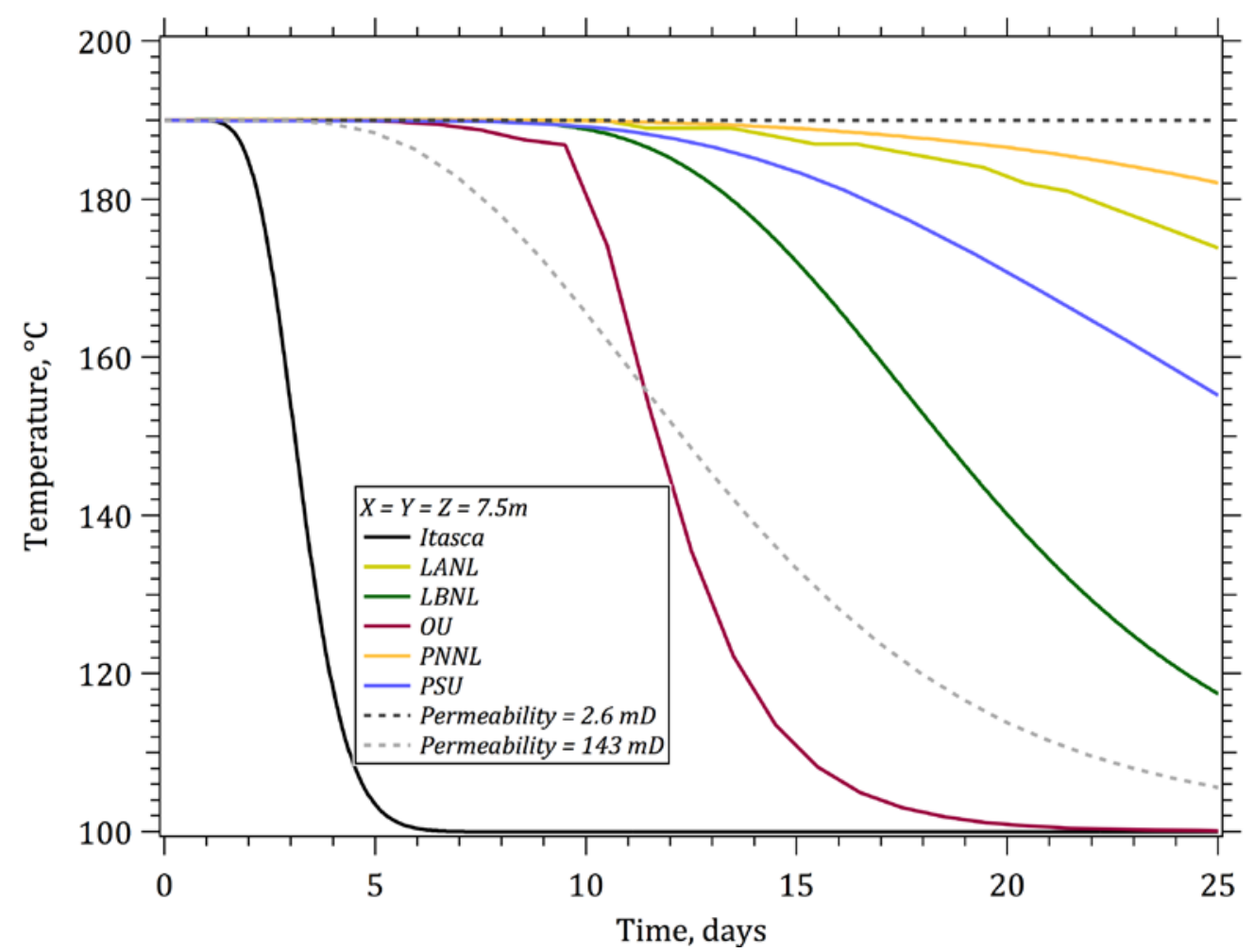

Figure 6-7. Simulation results for temperature versus time at $\mathrm{x}=\mathrm{y}=\mathrm{z}=7.5 \mathrm{~m}$

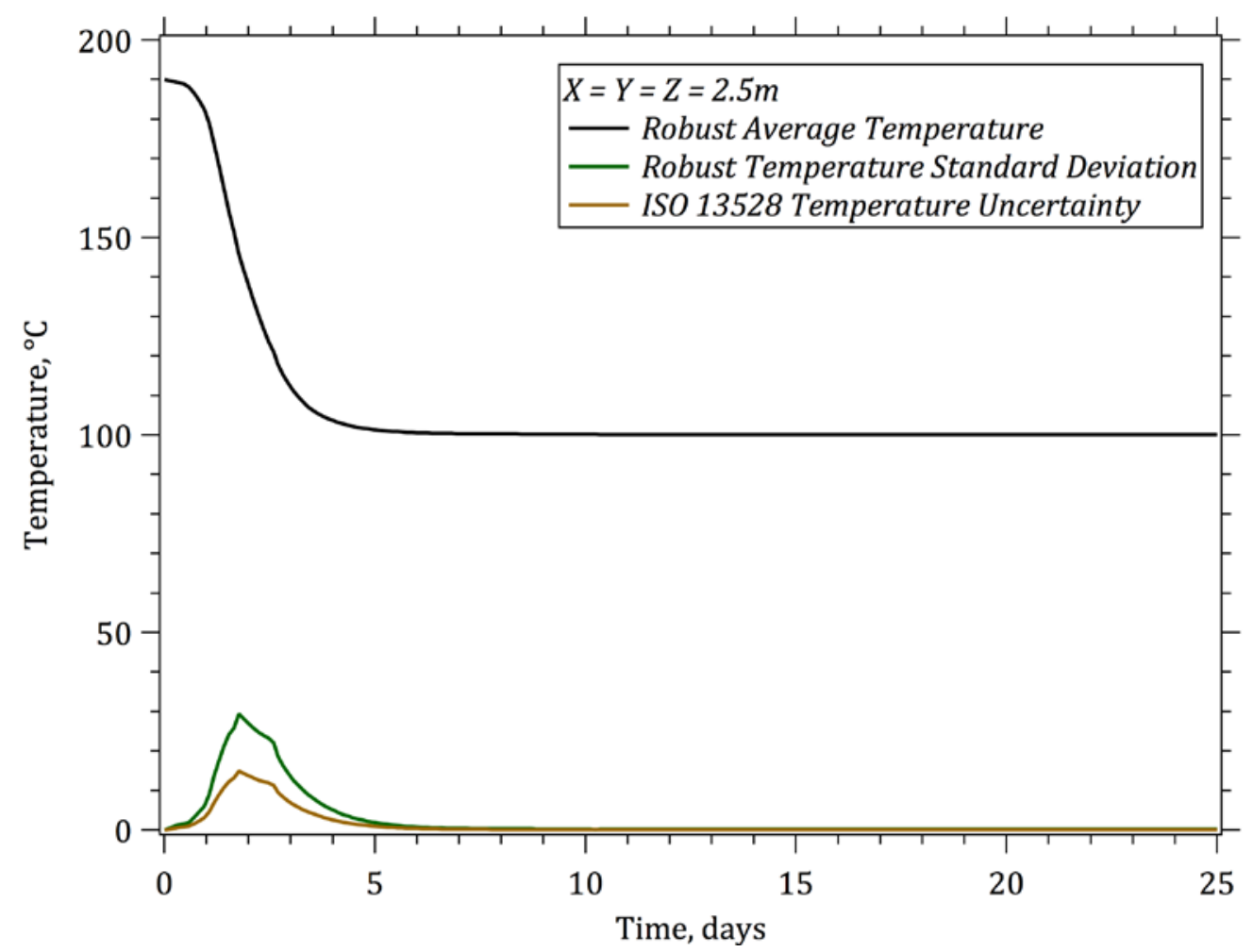

Figure 6-8. ISO 13528 uncertainty for temperature versus time at $\mathrm{x}=\mathrm{y}=\mathrm{z}=2.5 \mathrm{~m}$ 


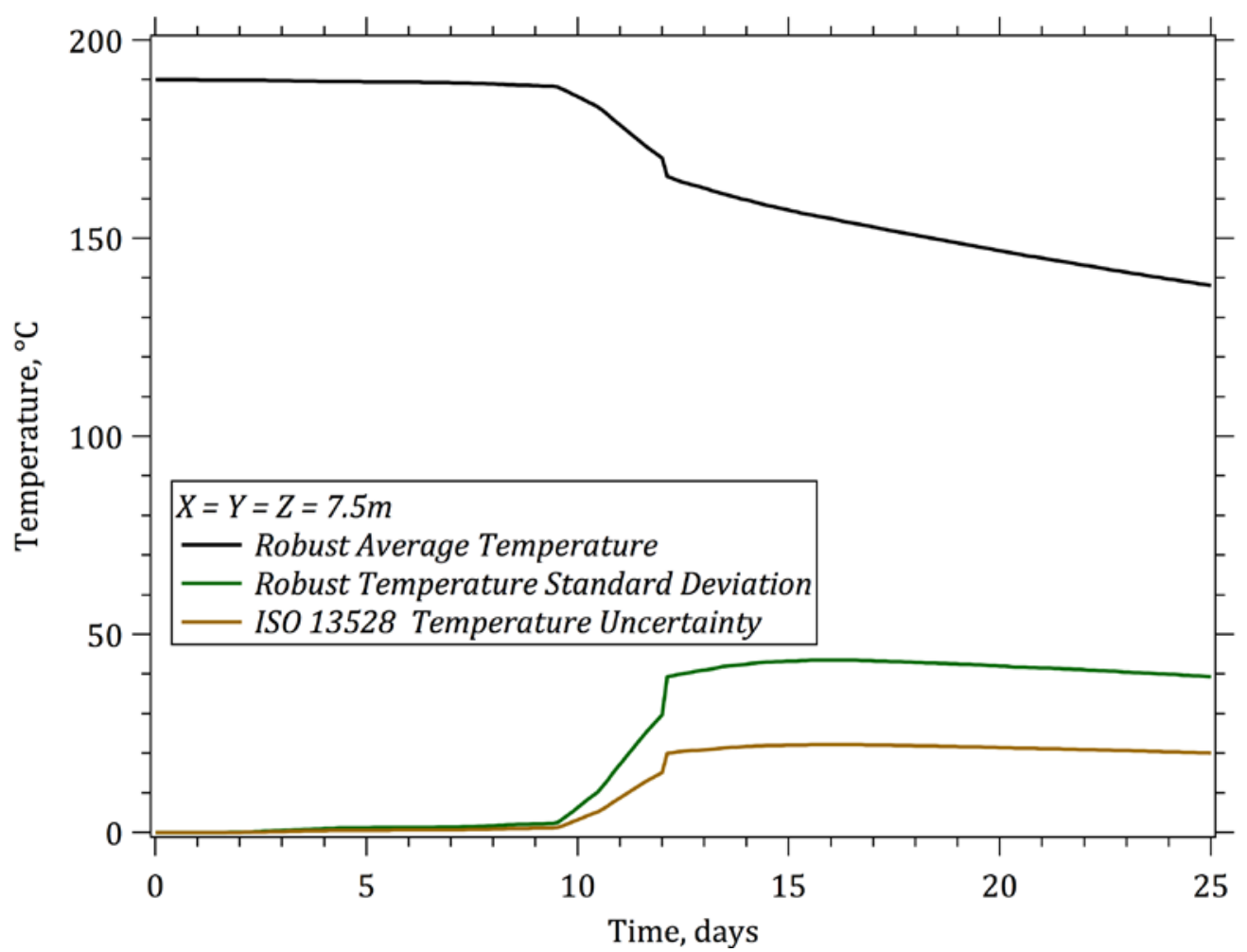

Figure 6-9. ISO 13528 uncertainty for temperature versus time at $\mathrm{x}=\mathrm{y}=\mathrm{z}=7.5 \mathrm{~m}$

Developments of the MCStress (Eqn. (6.1)) as a function of time at the point $\mathrm{x}=\mathrm{y}=\mathrm{z}=2.5 \mathrm{~m}$ from the injection point predicted by the various models (with the exception of the OU model) are shown in Fig. 610. All models show the trend of MCStress starting with a negative value, and rising to zero in less than 4 days and then plateauing at a positive value between 11-21 MPa in less than 9 days. Negative values of the MCStress denote a stable rock state prior to reaching the failure threshold. Rock failure occurs as MCStress crosses the zero value to become positive. Increasing positive values of MCStress are a modeling artifact caused by the fact that none of the models include plasticity in the stress calculations. The positive values of MCStress serve as a computational proxy for material damage, which in these models is represented by modified permeability as given in Eqn. (6.2). The Itasca model shows the fastest rise, followed by the LBNL, PSU, PNNL and LANL models, in that order. These trends are generally consistent with the cooling trends shown in Fig. 6-5. The PSU and PNNL models show the highest MCStress buildup, while the LANL and LBNL models show the lowest. Similar trends are noted in Fig. 6-11 showing the MCStress at the point $\mathrm{x}=\mathrm{y}=\mathrm{z}=7.5 \mathrm{~m}$. 


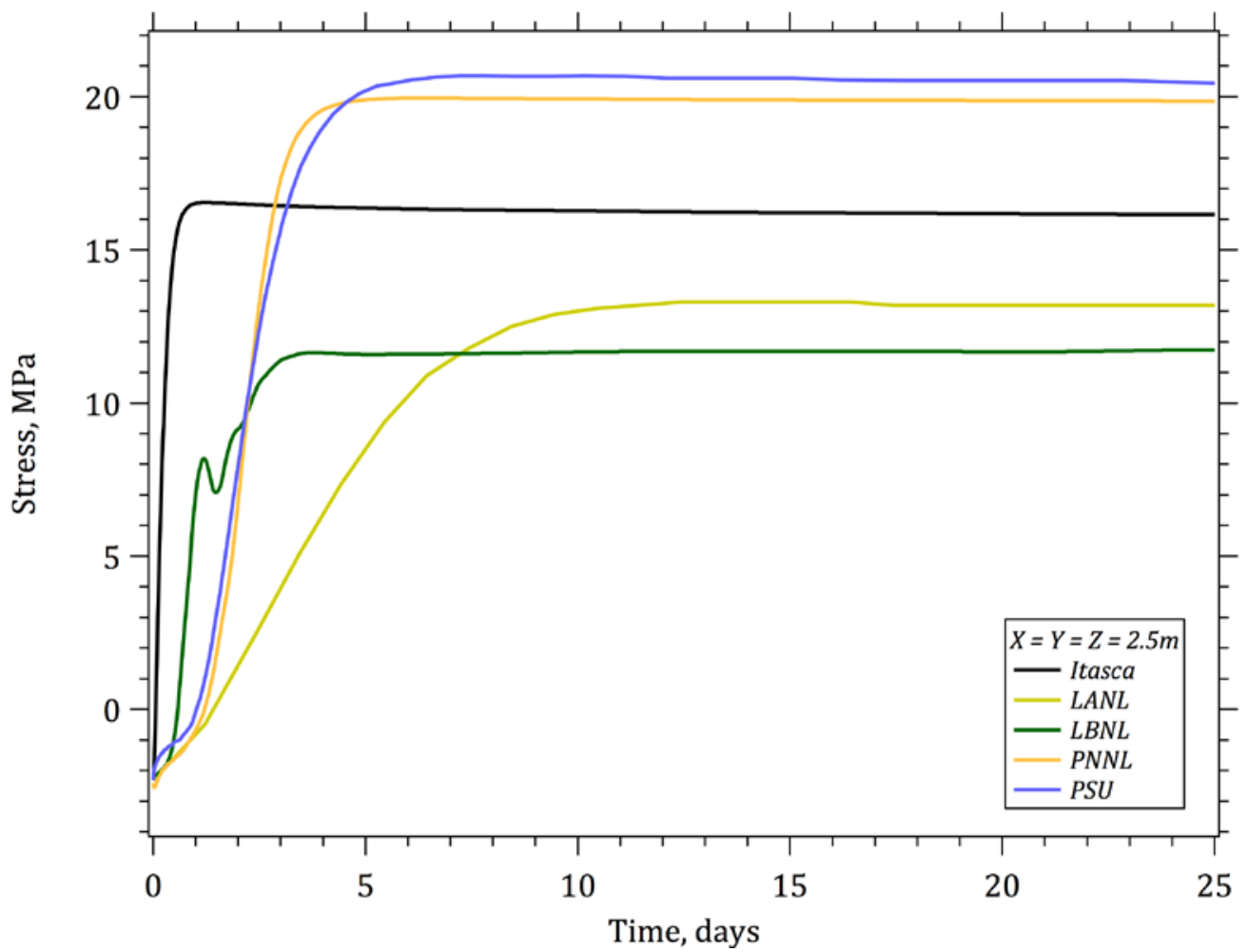

Figure 6-10. Simulation results for stress versus time at $x=y=z=2.5 \mathrm{~m}$

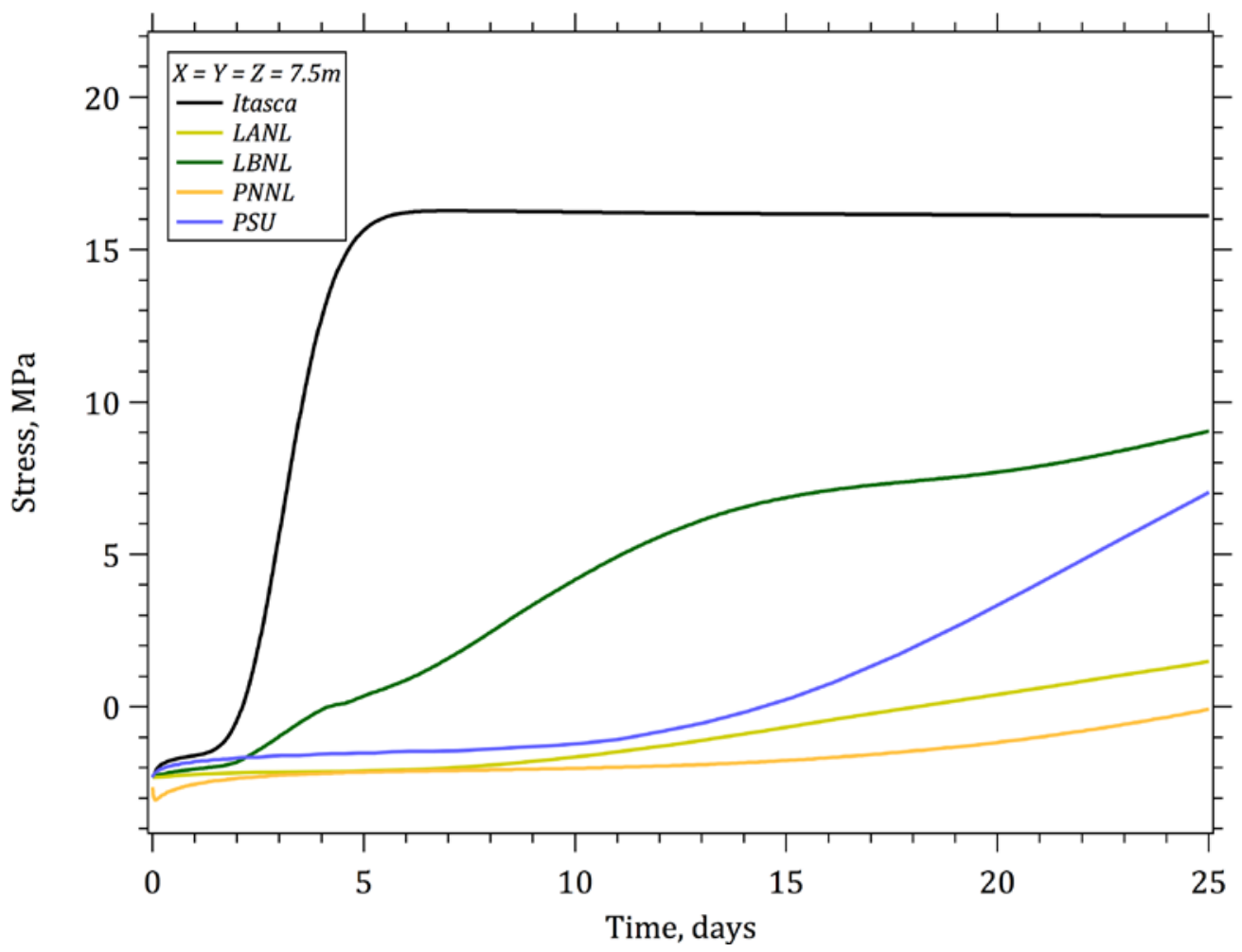

Figure 6-11. Simulation results for stress versus time at $x=y=z=7.5 \mathrm{~m}$ 


\subsection{Discussion of Benchmark Problem 2 Results}

Comparison of results between groups shows broad agreement, for the injection flow rate and temperature variation with time close to the injection point, although the discrepancy appears to increase with distance. Matches between the codes are imperfect because of the different numerical approaches used by the groups and due to the different constitutive relations applied in the different models. Considering the practical importance of the stimulation scenarios represented by this test problem and the differences experienced between the available models, future efforts are warranted for reconciliation. It would be instructive to attempt the derivation of a semi-analytical solution to the problem under appropriate realistic simplifying assumptions for verification. This would facilitate a more detailed comparison between the results of various models. 



\subsection{Benchmark Problem 3: Fracture Opening and Sliding in Response to Fluid Injection}

\section{Problem Champion: Mark McClure, The University of Texas at Austin}

The objective of this problem is to simulate injection into a system with three fractures. The problem is based on simulations performed by (McClure 2012) and (Mutlu and Pollard 2008). Injection is performed into a central fracture oriented to slide in response to increased fluid pressure. The central fracture is connected to two peripheral fractures perpendicular to the minimum principal stress (Fig. 7-1). Leakoff of fluid from the fractures to the surrounding matrix is neglected. Injection is performed at constant pressure until the fluid pressure in all three fractures has become constant. The injection pressure is less than the minimum principal stress.

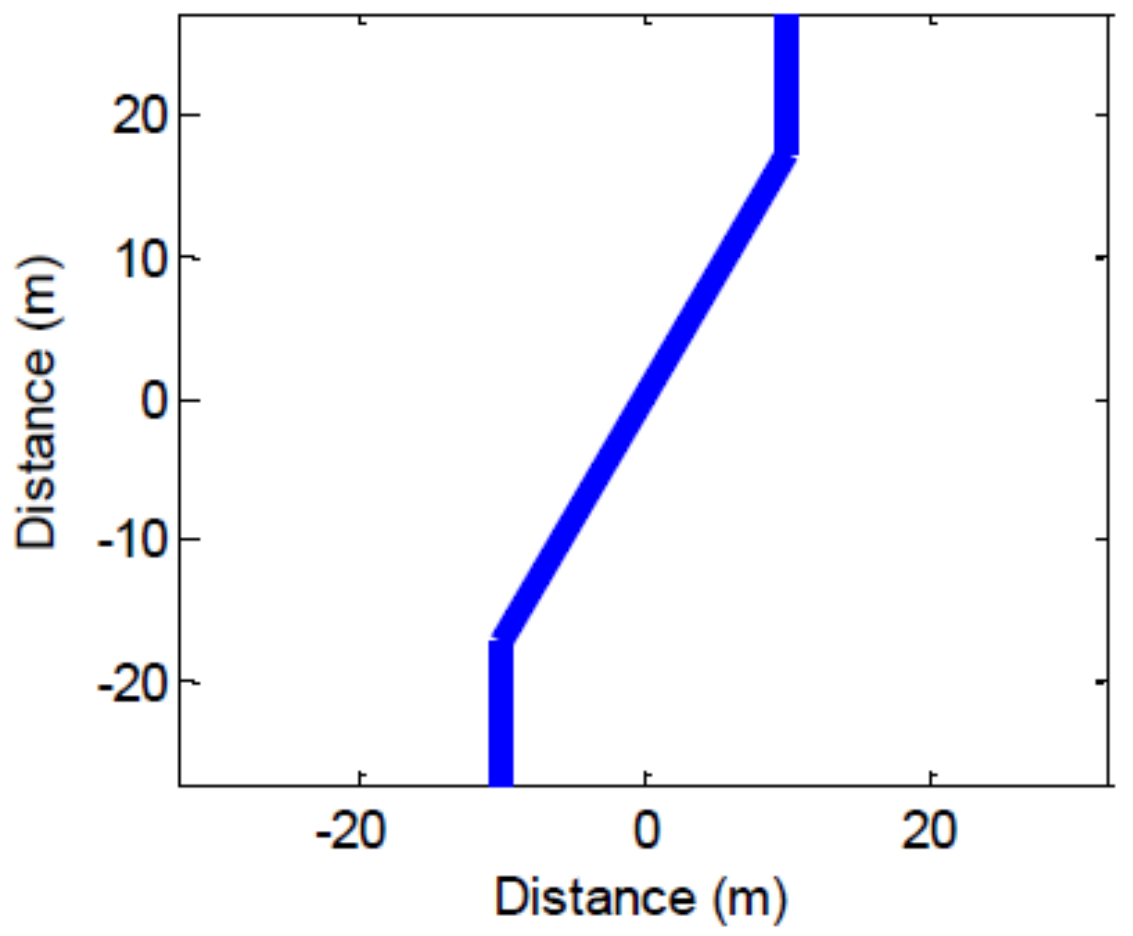

Figure 7-1. Problem geometry for the base case with three preexisting fractures

The stresses induced by the deformation of the fractures have strong effects on the simulation results. Stresses induced by the sliding of the central natural fracture induce tension on the peripheral fractures, causing them to partially open, even though fluid pressure remains below the minimum principal stress. The peripheral fractures slide in response to shear stresses induced by the sliding of the central fracture. Results are compared on the basis of the final distribution of deformation (when fluid pressure has become constant everywhere) and on the basis of the evolution of fluid pressure, injection rate, and deformation with time.

This problem is relevant to EGS because it has been hypothesized that hydraulic fractures propagate off sliding natural fractures during stimulation (Jung 2013; McClure 2012; McClure and Horne 2014). The concentration of tensile stress created by the fracture sliding could enable hydraulic fractures (Mode I) to 
form even if the injection pressure is less than the minimum principal stress. This problem is a simplified demonstration of that process.

Nine teams participated in the solutions using various simplifying assumptions, multiple runs, and HM models as listed in Table 7-1. The codes used for the simulations are described in (White et al. 2015a).

Table 7-1. Participating Teams and Simulators Used in the Solution of Benchmark Problem 3

\begin{tabular}{lll}
\hline Simulation Team & Team Identifier & Code(s) \\
\hline Idaho National Laboratory & INL & FALCON \\
Itasca Consulting Group & Itasca & FLAC $^{3 D}$ \\
Lawrence Berkeley National Laboratory & LBNL & TOUGH and FLAC \\
Lawrence Livermore National Laboratory & LLNL & GEOS \\
Pennsylvania State University & PSU & FLAC3D and TOUGHREACT \\
Stanford University & Stanford & CFRAC and GPRS \\
University of Nevada, Reno & UNR & MULTIFLUX and TOUGH2 \\
The University of Oklahoma & OU & GEOFRAC \\
The University of Texas at Austin & UTA & CFRAC UT \\
\hline
\end{tabular}

\subsection{Geometry and Input Data for Benchmark Problem 3}

The geometry of the 3-fracture system is shown in Fig. 7-1. For the flow calculations, an out-of-plane thickness, $\mathrm{h}=100 \mathrm{~m}$, for the fractures is assumed (so that the elements do not have infinite volume). Simplifying assumptions are:

- Isothermal

- Single phase, single component fluid

- Constant fluid viscosity

- Constant fluid compressibility

- No leakoff from the fractures

- Constant fracture transmissivity, even for open fractures (walls out of contact)

- No chemical effects

- Stresses induced by the very slight normal displacements of closed fracture elements are neglected

The fractures are assumed to have a constant compressibility so that the fracture aperture of a closed element can be given by the simple equation:

$$
E=E_{r e f} \exp \left(c_{E}\left[\sigma_{n}-P\right]\right)
$$

where $E$ is aperture (volume of fluid stored per surface area of fracture), $\sigma_{n}$ is normal stress, $c_{E}$ is the fracture compressibility and $P$ is fluid pressure. For an open fracture element, the aperture is given by the equation:

$$
E=E_{\text {ref }}+E_{\text {open }}
$$

where $E_{\text {open }}$ is the mechanical opening of the fracture. Because the fracture transmissivity is assumed constant, the hydraulic aperture is effectively assumed to be constant. Although constant fracture transmissivity is not realistic, this assumption was made in order to simplify the problem and facilitate comparison between codes. 
The properties of the reservoir rock are presented in Table 7-2.

Table 7-2. Material Properties

\begin{tabular}{lll}
\hline Property Name & Units & Value \\
\hline Shear Modulus, $G$ & $\mathrm{MPa}$ & 15000 \\
Poisson's ratio, $v$ & -- & 0.25 \\
Out-of-plane thickness, $h$ & $\mathrm{~m}$ & 100 \\
Fracture Coefficient of Friction, $\mu$ & -- & 0.6 \\
Fracture Cohesion, $S_{0}$ & -- & 0 \\
Fracture Transmissivity, $T_{f}$ & $\mathrm{~m}^{3}$ & $10^{-13}$ \\
Fracture Compressibility, $c_{E}$ & $1 / \mathrm{MPa}$ & 0.01 \\
Reference Aperture, $E_{\text {ref }}$ & $\mathrm{m}$ & .001 \\
Fluid Viscosity, $\mu_{w}$ & $\mathrm{cp}$ & 1.0 \\
Fluid Density (at initial fluid pressure), $\rho_{w}$ & $\mathrm{~kg} / \mathrm{m}^{3}$ & 1000.0 \\
Fluid Compressibility, $C_{w}$ & $1 / \mathrm{MPa}$ & 0.00458 \\
\hline
\end{tabular}

\subsection{Initial and Boundary Conditions and Sources for Benchmark Problem 3}

The fractures are initially saturated with fluid at a pressure of $18 \mathrm{MPa}$. The initial stress state is assumed to be homogeneous, with $\sigma_{x x}$ equal to $21 \mathrm{MPa}$ and $\sigma_{y y}$ equal to $26 \mathrm{MPa}$. It is assumed that any stress caused by the formation of the preexisting fractures is negligible, so that the normal and shear stress on the fractures can be calculated solely by resolving the remote stress field onto the preexisting fractures, and the initial aperture can be calculated directly using Eqn. (7.2). Injection is performed at a constant pressure of 20.25 MPa. The simulation is performed for one week, long enough that the fluid pressure everywhere in the three fractures reaches $20.25 \mathrm{MPa}$.

\subsection{Physical processes and governing equations for Benchmark Problem 3}

The simulations have no fracture propagation (the fractures are preexisting) and include the following physical processes and properties:

1. unsteady state mass balance coupled with Darcy's law

2. linear elastic deformation of the material around the fractures (satisfying quasistatic stress equilibrium, Hooke's law, and the compatibility equations)

3. when fluid pressure on a fracture is less than the normal stress, the fracture is "closed;" closed fractures slide when their shear stress reaches their frictional resistance to slip, as defined by Coulomb's law:

$$
|\tau| \leq\left[\sigma_{n}-P\right] \mu+S_{o}
$$

where $\tau$ is shear stress, $\mu$ is the coefficient of friction, and $S_{o}$ is the cohesion.

4. when fluid pressure reaches the normal stress, fractures open (walls lose contact); open fractures have fluid pressure equal to their normal stress and do not bear any shear stress,

5. stress calculations are two- dimensional and plane strain 


\subsection{Solution Metrics for Benchmark Problem 3}

1. Plots of change in fracture aperture along the fractures (from the initial aperture) after one minute and at the end of the simulation (plotted as $y$-coordinate location versus change in aperture).

2. Plots of fracture sliding along the fractures after one minute and at the end of the simulation (plotted as y-coordinate location versus sliding).

3. Plots of injection rate versus time.

\subsection{Results for Benchmark Problem 3}

As seen in Fig. 7-2, the injection rate is highest at the beginning of the simulation and gradually declines to zero over time. At the start of the simulation, there is a sharp pressure difference between the well (which injects at a constant pressure) and the adjacent fracture. Because there is no leakoff into the matrix, the fluid pressure eventually rises to equal the injection pressure everywhere, and the injection rate goes to zero. The PSU and LBNL results have a lower rate than the other simulation results at all times, suggesting that there is less cumulative fluid injection in these simulations than in the others, probably caused by differences in how fluid storage is calculated. Both UNR results deviate significantly from the general trend of the other results. The Itasca result has one of the lowest injection rates at early time, and the highest rate at late time, suggesting that a similar cumulative volume of fluid is injected, but there is some difference in the way fracture transmissivity is calculated. The OU result deviates significantly from the other results during the first 100 seconds, before settling onto a very similar trend. This is probably because the OU result started the simulation with comparatively large timesteps (around 30 seconds). The ISO 13528 uncertainty for injection rate versus time is shown in Fig. 7-3. The uncertainty in model results is more pronounced for this problem that many of the others, resulting from differences in timestep selection and how the models calculate storage.

The aperture results after 60 seconds of injection are quite variable (Fig. 7-4). These results are affected by the time-dependent process of fluid pressure spreading through the fractures. At 60 seconds, the region where significant aperture change occurs is small, and the gradient in aperture is significant. Some of the simulations are not performed with adequate mesh refinement to capture this behavior in detail. The PSU result has more opening along the peripheral fractures than the other results. The UNR Run 2 result matches several other simulations in maximum opening near the juncture, but predicts significantly more opening along the length of the peripheral fractures. As with the other results for Benchmark Problem 3, the Stanford and UTA results are nearly identical, with the Stanford curve overlaying and obscuring the UTA result on the figure. This is not surprising because the groups used different versions of a common base code. Fig. 7-5 shows the ISO 13528 uncertainty for the change in fracture aperture, and the distinct differences between models can be seen in the uncertainty particularly at the peak fracture opening. 


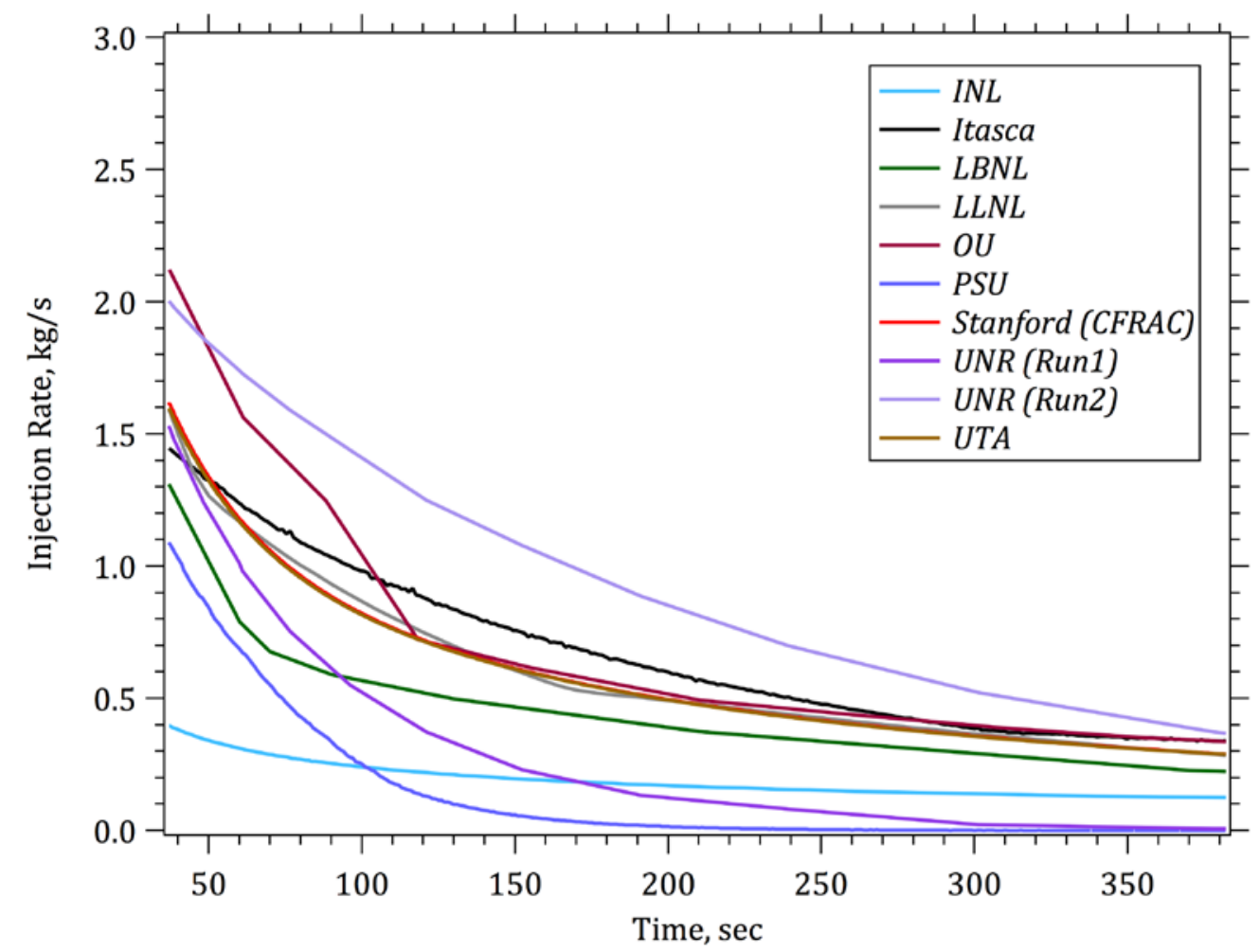

Figure 7-2. Simulation results for injection rate versus time

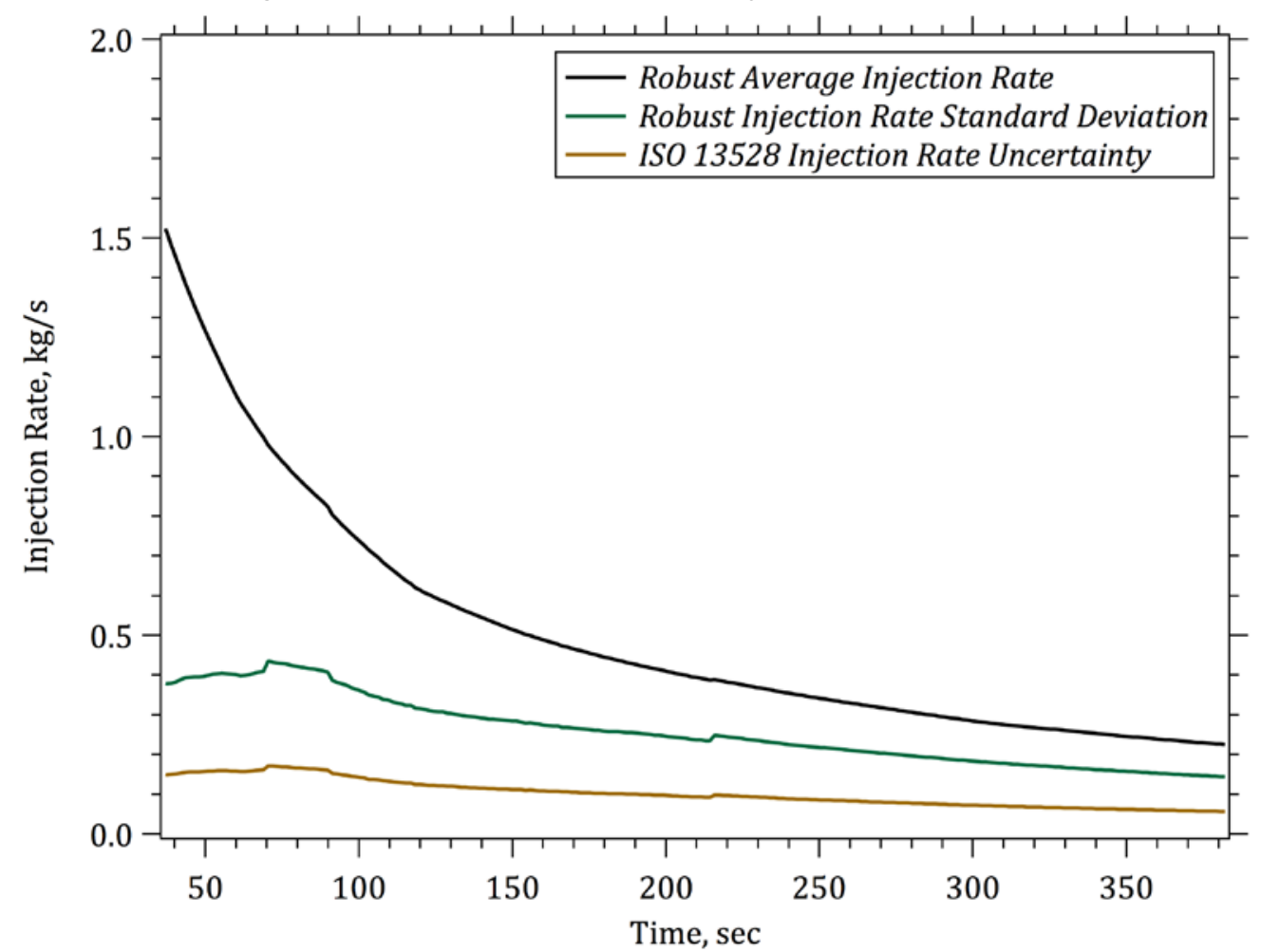

Figure 7-3. ISO 13528 uncertainty in injection rate versus time 


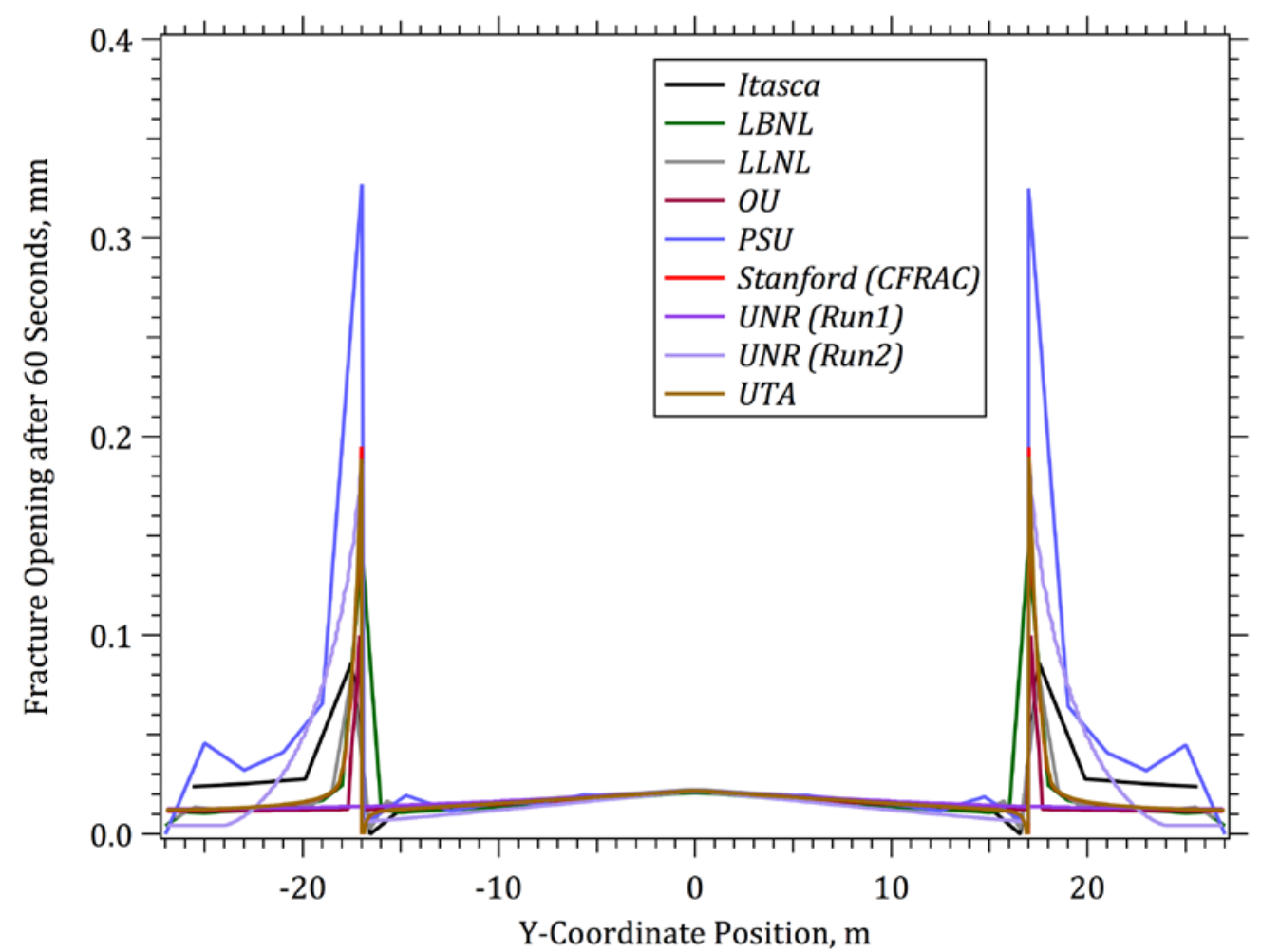

Figure 7-4. Simulation results for change in fracture aperture (from the initial aperture) after 60 seconds of injection

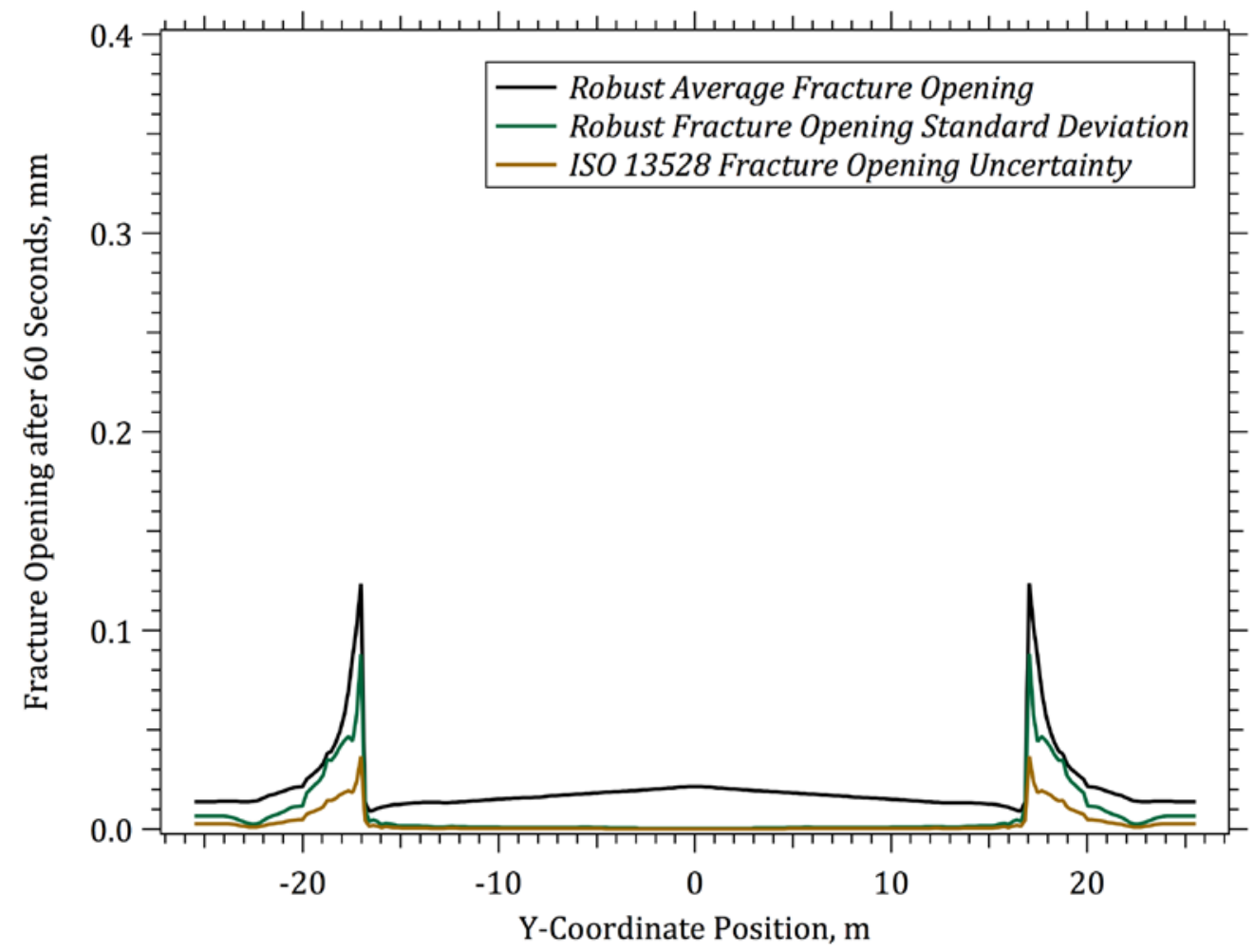

Figure 7-5. ISO 13528 uncertainty in change in fracture aperture after 60 seconds of injection 
The predicted final change in fracture aperture from UTA, Stanford, OU, LBNL, LLNL, and UNR Run 2 are quite similar (Fig. 7-6). The main point of difference occurs at the juncture between the central and peripheral fracture at $y= \pm 17$. Fracture opening sharply increases on the peripheral fractures near the juncture. This sharp gradient creates a strong mesh dependence that cannot be captured fully by the groups using a relatively coarse mesh (such as in the LLNL and Itasca submissions). The UTA, Stanford, and OU groups have nearly identical solutions for the final distribution of opening and sliding, and their results show the sharpest increase in aperture near the fracture juncture. These three codes use the boundary element method, which is especially well suited for this particular problem because of the sharp gradient in aperture in a small region due to a localized concentration of stress. The UNR Run 2 is tuned to match the UTA result for final opening displacement, and so the results match very closely. The UNR Run 1 result neglects the effect of sliding on aperture and therefore does not show any perturbation in aperture near the fracture juncture. The PSU result has significantly larger opening displacement along the length of the peripheral fracture, though the maximum displacement calculated at the juncture is similar to other results. ISO 13528 uncertainty for the change in fracture aperture at the end of the simulation shown in Fig. 7-7 demonstrates that the disparity in model assumptions and approaches results in significant differences in results.

The sliding results at 60 seconds are fairly similar between the groups (Fig. 7-5), except that the PSU result predicts significantly more sliding on the peripheral fractures than the other results. This is evident in Fig. 7-9 which shows the ISO 13528 uncertainty to be nearly zero. For the final fracture sliding distribution, all results are similar (Fig. 7-10). The LLNL and PSU results have modestly less sliding along the fractures than the other submitted results. This is evident in Fig. 7-11 which shows the ISO 13528 uncertainty to be nearly zero.

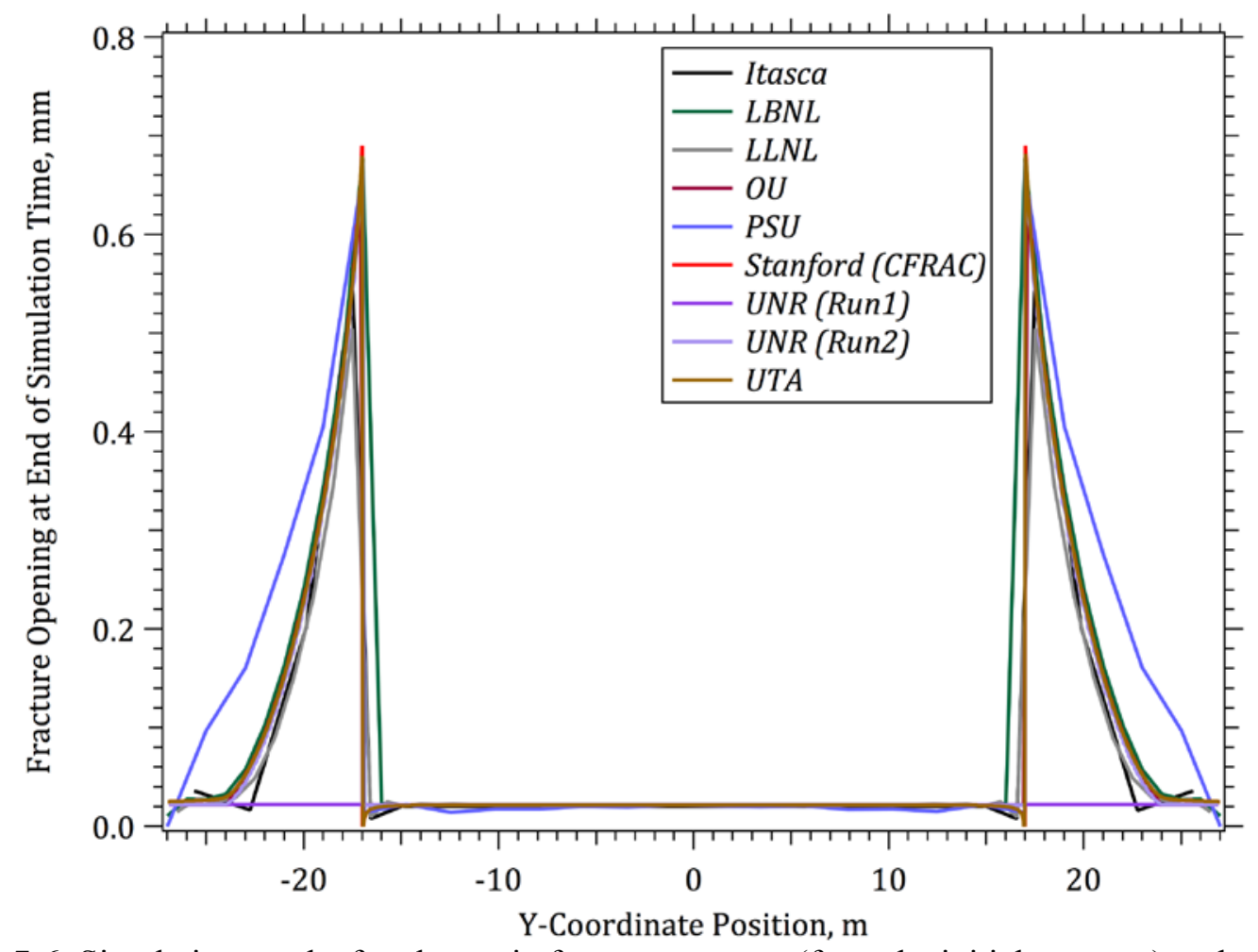

Figure 7-6. Simulation results for change in fracture aperture (from the initial aperture) at the end of the simulation 


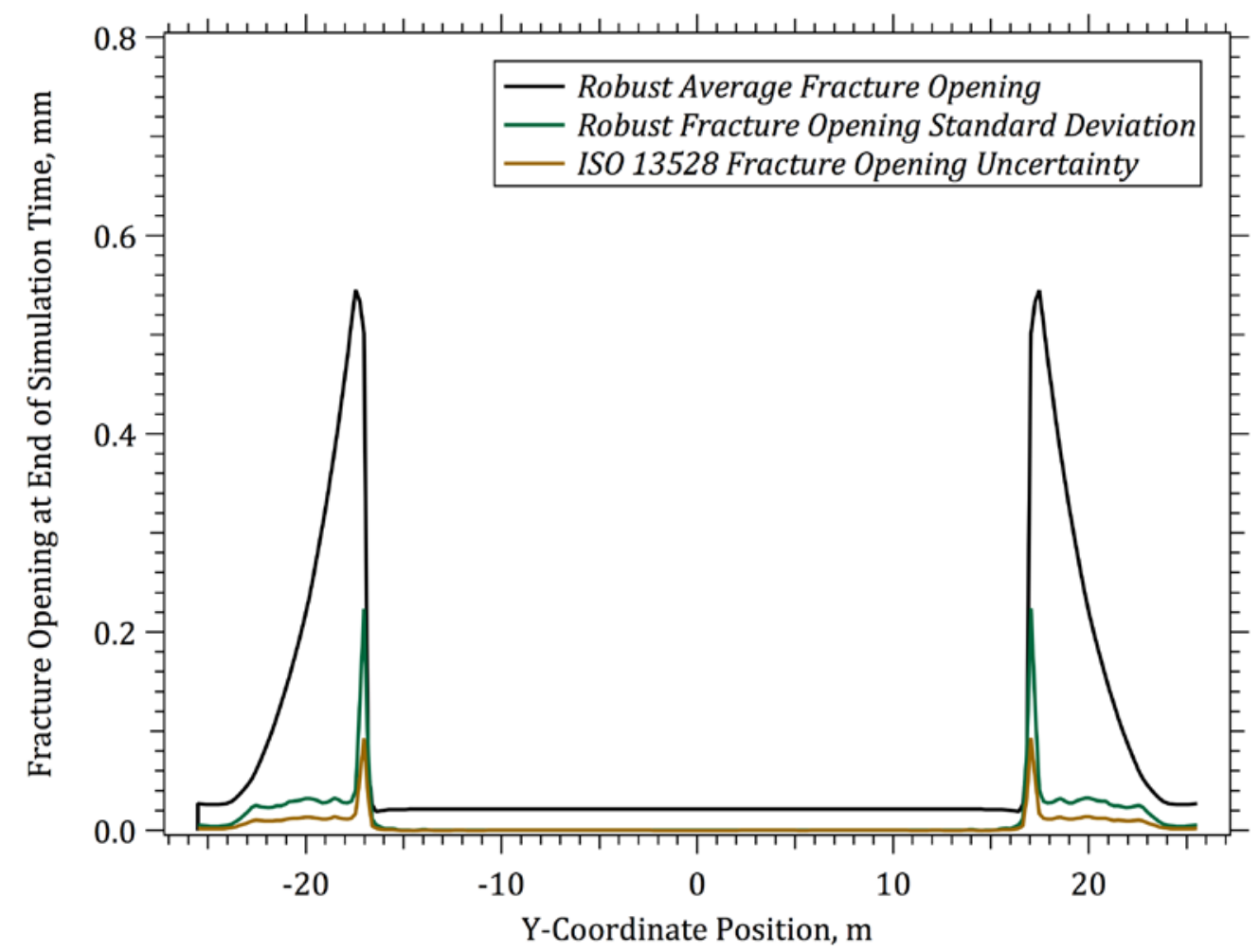

Figure 7-7. ISO 13528 uncertainty in change in fracture aperture at the end of the simulation

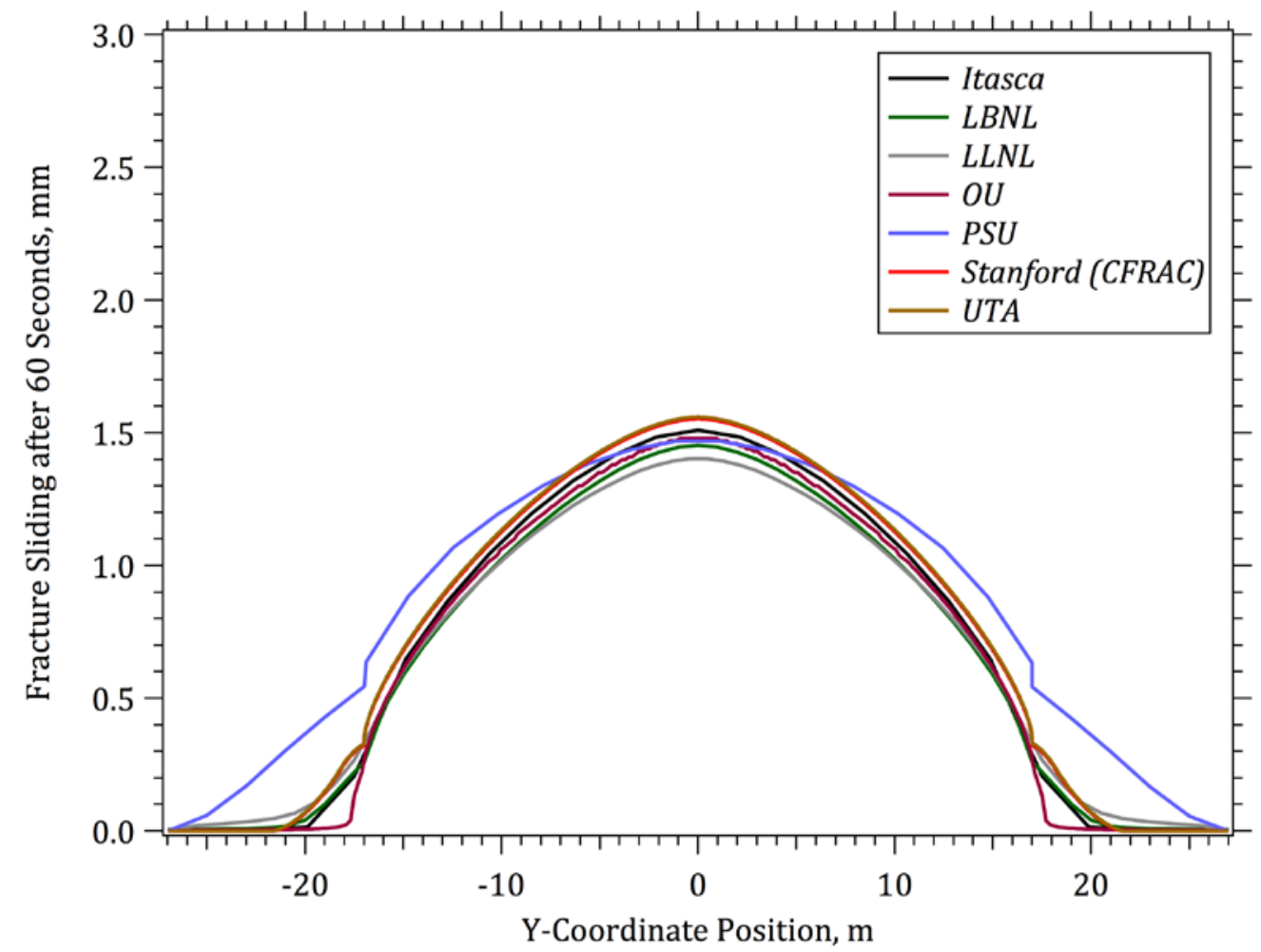

Figure 7-8. Simulation results for fracture sliding after 60 seconds of injection 


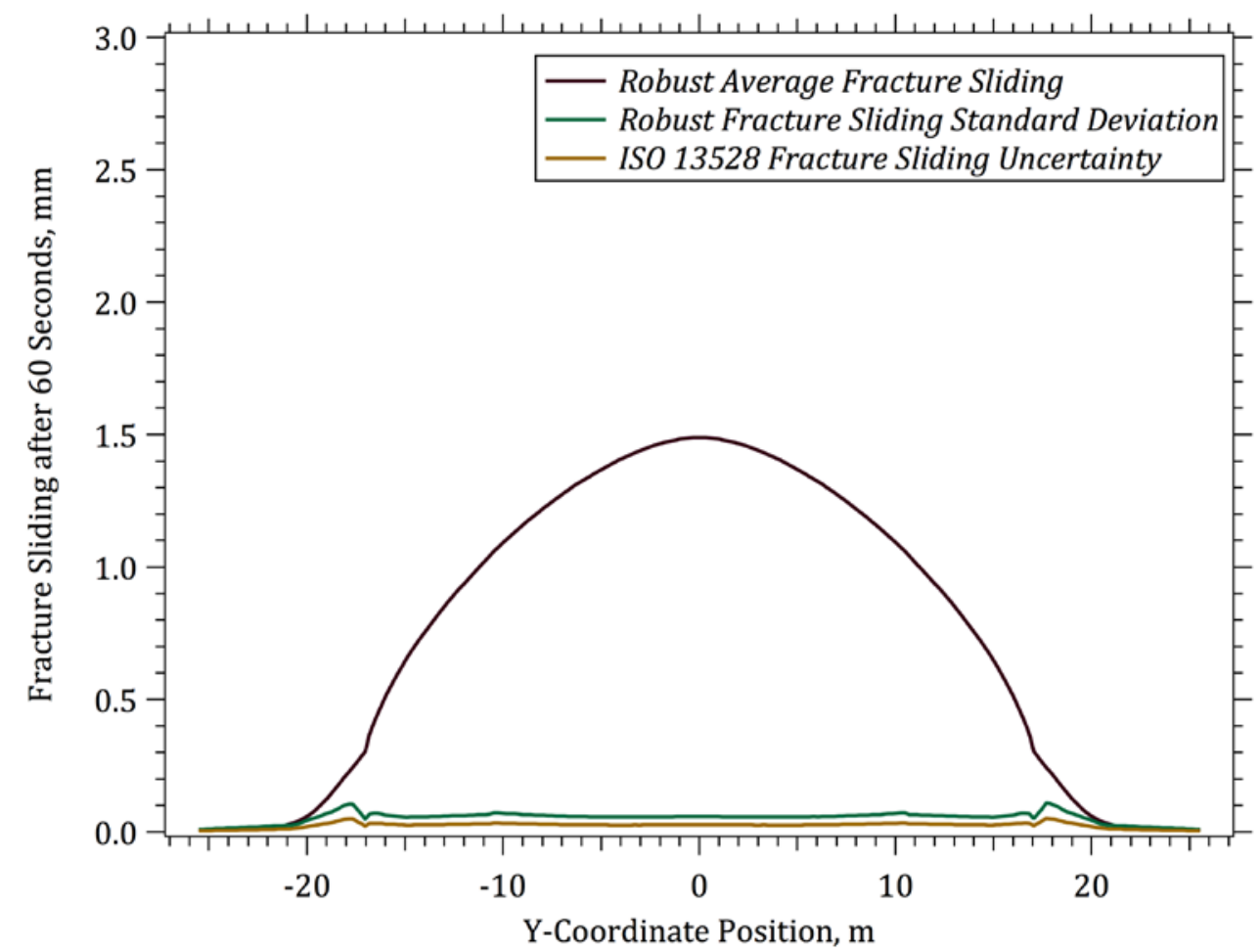

Figure 7-9. ISO 13528 uncertainty in fracture sliding after 60 seconds of injection

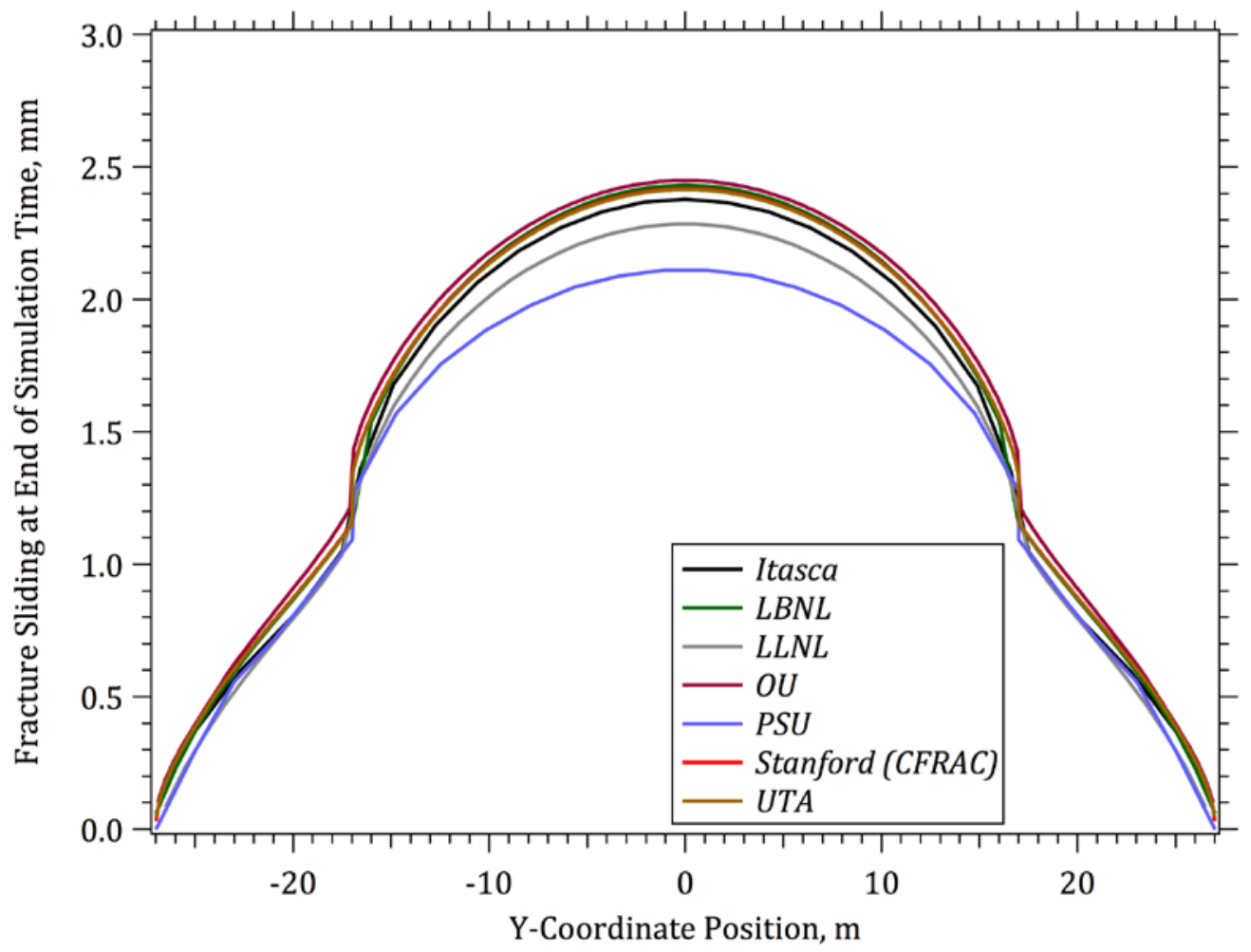

Figure 7-10. Simulation results for fracture sliding at the end of the simulation 


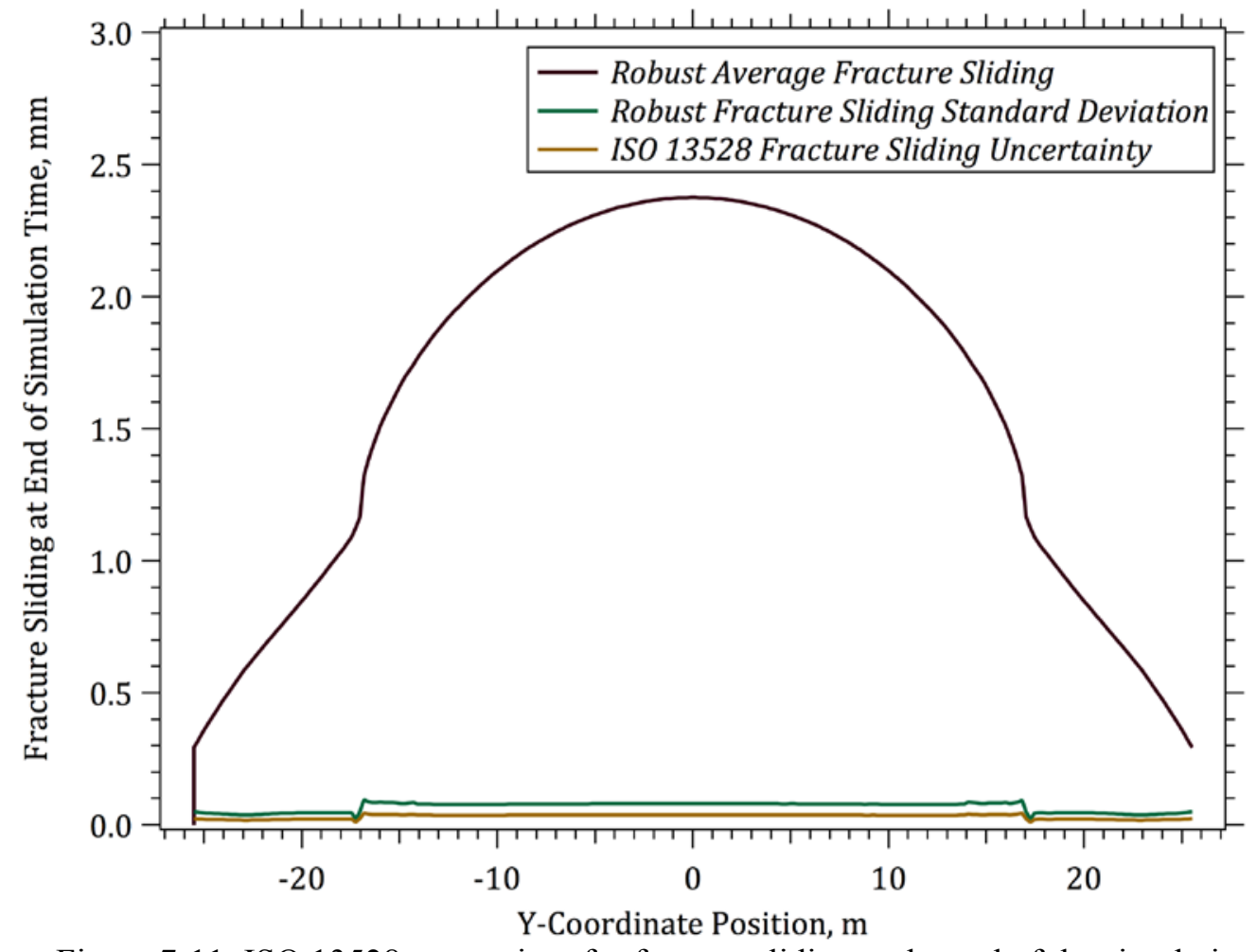

Figure 7-11. ISO 13528 uncertainty for fracture sliding at the end of the simulation

\subsection{Discussion of Benchmark Problem 3 Results}

Because the fluid pressure is uniform everywhere in the fractures at the end of the simulation, the final distributions of deformation are determined solely from a static deformation problem. The increase in fluid pressure along the central fracture causes it to slide. The peripheral fractures initially bear zero shear stress, but the sliding of the central fracture induces shear stress on the peripheral fractures, causing them to slide as well. The sliding of the central fracture also reduces the normal stress on the peripheral fractures, causing them to mechanically open near their juncture with the central fracture. The fluid pressure remains below the minimum principal stress, but locally, the fluid pressure exceeds the normal stress on the peripheral fractures at the juncture. The same trends in deformation are apparent at one minute, but are less pronounced because the fluid pressure is still in the process of increasing inside the fractures.

Overall, there is a qualitative, but not exact, match between the different simulation results. There is a theoretically "exact" solution to this problem, but it is not known, and so we do not have an independent, preexisting solution by which to determine the most accurate results. Some differences between the results are clearly due to differences in mesh refinement. However, some results deviate so significantly from the others that the differences must be due to differences in accuracy or the way the problem was set up by the groups.

The static deformation problem, isolated from time-dependent effects, is solved with reasonable accuracy by the groups, as seen from comparison of the final fracture opening and sliding results. Differences are mostly at the fracture juncture and are apparently due to differences in mesh refinement. However, the time-dependent effects show considerable variability between groups. Correspondingly, the ISO 13528 robust standard deviation is a significant percentage of the robust average (indicating lower agreement 
between groups) for the injection rate, the change in fracture aperture after $60 \mathrm{~s}$, and for the final change in fracture aperture near the juncture.

Most likely, the mismatch in time dependent effects is due to difficulty in implementing constitutive relations for fracture aperture and transmissivity consistently across all groups. This problem uses rather unusual relations for fracture aperture and transmissivity (Eqns. (7.1) and (7.2) for aperture and a constant specified value for transmissivity). These simple relations are used in order to facilitate consistency across groups. There is not a universally accepted set of constitutive relations available for describing fracture aperture and transmissivity as a function of fracture aperture and sliding deformation. As a result, regardless of which constitutive relations are chosen, some of the codes will not already have those relations implemented. This underscores the value of developing codes with the flexibility to easily implement user-defined constitutive relations. This also suggests that work is needed to synthesize the rock mechanics literature on fracture aperture and transmissivity and identify which relations would be most appropriate for universal adoption among code developers. 



\title{
8.0 Benchmark Problem 4: Planar EGS Fracture of Constant Extension and Penny-Shaped or Thermo-elastic Aperture in Impermeable Hot Rock
}

\author{
Problem Champion: George Danko, University of Nevada, Reno
}

Benchmark Problem 4 considered the responses of a single planar fracture in the rock based on the first experimental EGS in the U.S. at Fenton Hill, Phase I. The thermal-hydrologic-mechanical (THM) responses of this fracture during a 24-day injection and production period were simulated. The rock mechanics model component includes the thermo-elastic response of the self-propped fracture layer coupled to the thermal model of the reservoir during coolant injection. Six teams participated in the solutions using various simplifying assumptions, multiple runs and THM models as listed in Table 8-1. The methods and codes used by each team are described in (White et al. 2015a).

Table 8-1. Participating Teams and Simulators Used in the Solution of Benchmark Problem 4

\begin{tabular}{lll}
\hline Simulation Team & Team Identifier & Code(s) \\
\hline Itasca Consulting Group & Itasca & FLAC \\
Lawrence Berkeley National Laboratory & LBNL & TOUGH and FLAC ${ }^{3 \mathrm{D}}$ \\
Lawrence Livermore National Laboratory & LLNL & GEOS \\
Pacific Northwest National Laboratory & PNNL & STOMP \\
University of Nevada, Reno & UNR & MULTIFLUX and TOUGH2 \\
The University of Texas at Austin & UTA & CFRAC_UT \\
\hline
\end{tabular}

Two sub-cases were applied to the problem:

1. In Case 1, temperature-dependent fluid properties and a constant-aperture, penny-shaped fracture is assumed. The injection temperature and flow rate are given. The objective is to match as closely as possible the measured production temperature at the extraction point and the pressure loss across the fracture between the injection point and production well for a 24-day experiment conducted at Fenton Hill, and for the entire period of 75 days.

2. In Case 2, the assumption about the fracture aperture is refined. A rock mechanics model component is added to include the elasticity of the self-propped fracture layer, expanding the task to THM modeling. The objective is to determine if the THM model can better match the measured production temperature at the extraction point and pressure loss across the fracture than the simple TH model.

\subsection{Geometry and Input Data for Benchmark Problem 4}

A penny-shaped, planar fracture (constant aperture) is defined with impermeable rock around it following as closely as possible the EGS arrangement at the Fenton Hill Phase I experiments. The key assumption is that the geometry of the planar fracture is known with a given constant fracture aperture, and a given constant radial extension in the plane. Note that an open fracture and not a porous layer are defined.

In Case 2, the radial extension of the planar fracture is constant as in Case 1, however, the aperture of fracture is assumed to change due to the elasticity and thermal dilatation of the rock. A simple, linear thermo-mechanical, elastic fracture aperture model may be used by calibration to the in situ measurement results as follows (Danko and Bahrami 2013a, b): 


$$
\delta(x, y)=\delta_{o}+C^{P}\left[\bar{P}(x, y)-\bar{P}_{o}(x, y)\right]+C^{T} \Delta \bar{L}\left[\bar{T}(x, y)-\bar{T}_{o}(x, y)\right]
$$

where $\delta$ is the hydrodynamic fracture aperture, $\delta_{0}$ is the initial fracture aperture, $C^{p}$ is the pressure aperture coefficient, $C^{T}$ is the thermal aperture coefficient, $p_{0}$ and $T_{0}$ are the initial pressure and temperature, $p$ and $T$ are the pressure and temperature, and $\Delta \bar{L}$ is the thermal contraction. However, other joint characteristics are used by some project participants. The LLNL model applies the Barton-Bandis exponential joint characteristics, while the UTA model uses the in situ stress as a threshold pressure for the unconfined fracture opening. The properties and the in situ virgin temperature of the rock are also known. The injection flow rate and temperature as a function of time likewise follow given trends from published data (Murphy et al. 1981). The injection and production boreholes are assumed to be heated and/or cooled by the surrounding rock mass during circulation.

The Fenton Hill Phase I reservoir model has an injection well situated at a depth of $2750 \mathrm{~m}$ below the surface, and a production well connected to the EGS fracture at a depth of $2630 \mathrm{~m}$. The injection well is connected $25 \mathrm{~m}$ from the bottom of a $120 \mathrm{~m}$ diameter fracture, an estimate given by (Murphy et al. 1981). Fig. 8-1 shows the conceptual diagram of the EGS arrangement. The depths of the injection and production wells are measured from the surface.

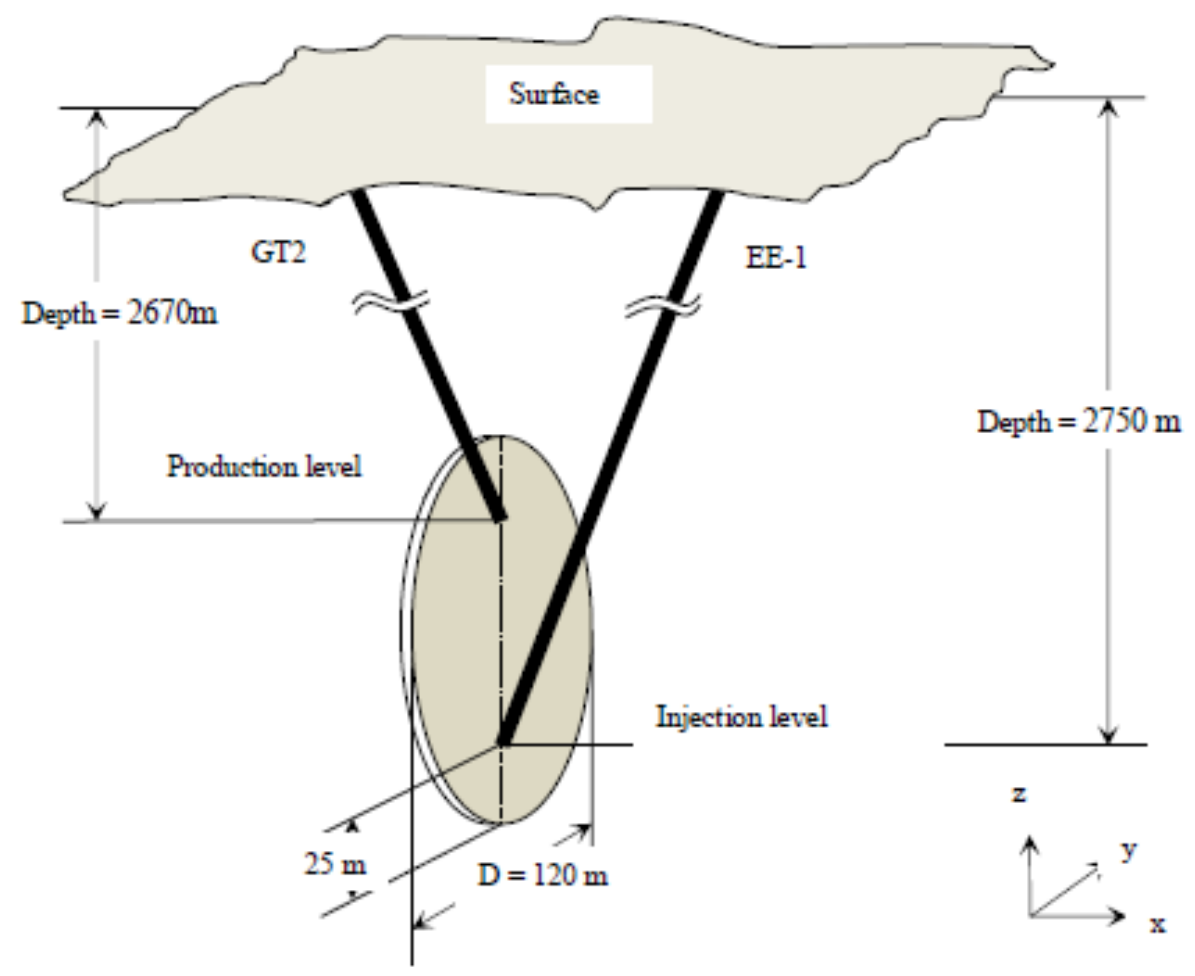

Figure 8-1. Simplified reservoir geometry (after (Murphy et al. 1981))

Fig. 8-2 shows the numerical model domain with the fracture at the center as a disc void space of $120 \mathrm{~m}$ in diameter. Note that the discretization is not shown to scale. The dashed line separates the near-field and the far-field domains. In Case 1 , the planar fracture aperture is assumed to be constant $(0.141 \mathrm{~mm})$, irrespective of fluid pressure and rock temperature. In Case 2 , the planar fracture aperture, $\delta_{0}$, without fluid injection is assumed to be nearly closed and constant $\left(1 \times 10^{-6} \mathrm{~m}\right)$; just enough to conduct fluid flow in a minute crack under pressure. The radial extension of the closed but conducting fracture is constant as in Case 1. The fracture aperture at the Fenton Hill Phase I reservoir is quantitatively not known. The given aperture of $0.141 \mathrm{~mm}$ in Case 1 is obtained from a trial-and-error simulation according to which the aperture is determined by matching the injection pressure between simulation and field measurement for 
the given injection flow rate at days 1 through 4 when the temperature is still unchanged in the reservoir. The properties of the reservoir rock are summarized in Table 8-2.

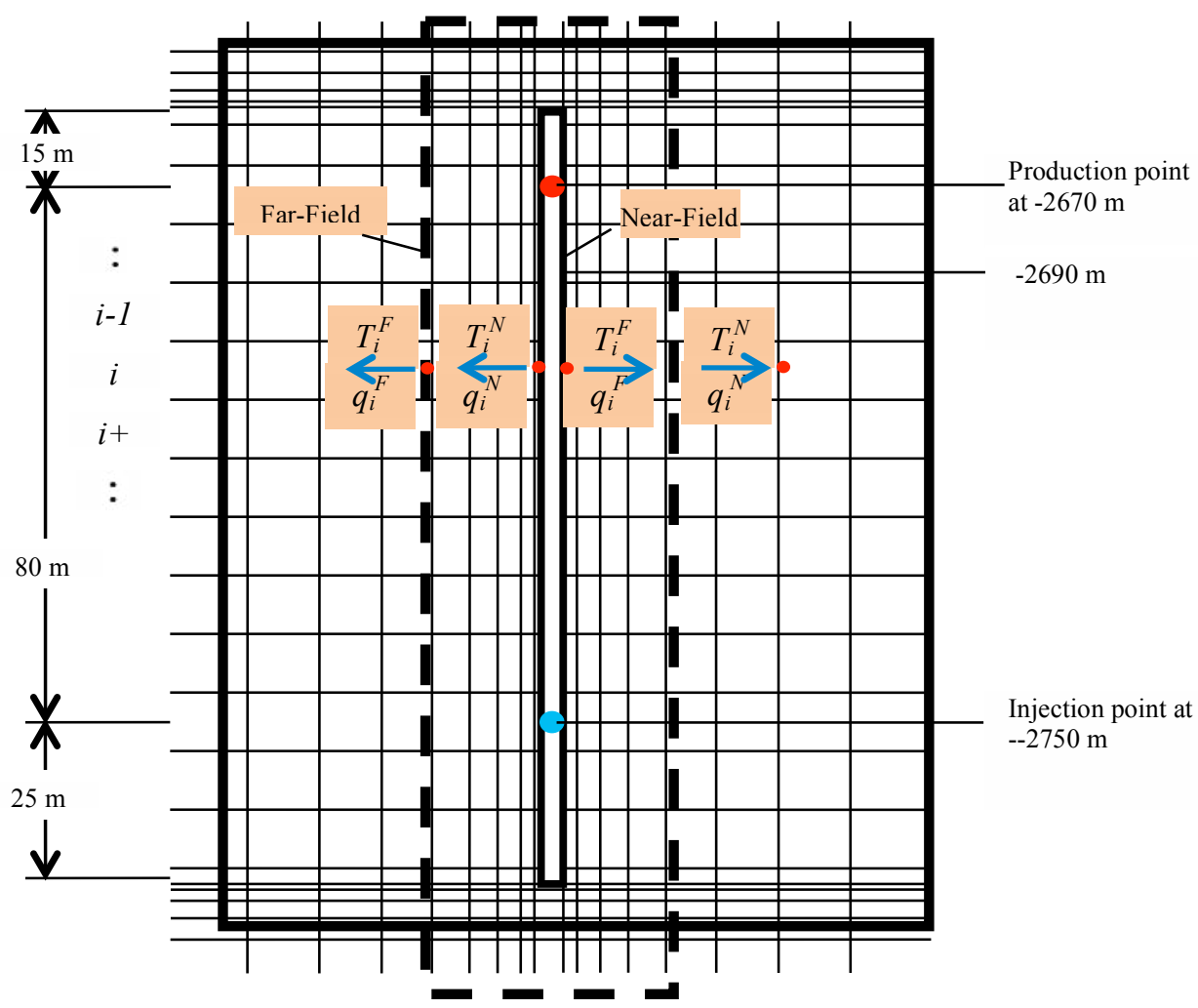

Figure 8-2. Schematic diagram of the near-field and far-field domains of Fenton Hill EGS with a $120 \mathrm{~m}$ diameter fracture

Table 8-2. Material Property Table for Benchmark Problem 4

\begin{tabular}{lll}
\hline Property Name & Units & Value \\
\hline Thermal Conductivity, $\kappa$ & $\mathrm{W} / \mathrm{m} \mathrm{K}$ & 2.9 \\
Heat Capacity, $c$ & $\mathrm{~J} / \mathrm{kg} \mathrm{K}$ & 0.25 \\
Density, $\rho$ & $\mathrm{Kg} / \mathrm{m}^{3}$ & 2700 \\
Diffusivity, $D$ & $\mathrm{~m}^{2} / \mathrm{s}$ & $1.0 \times 10^{-6}$ \\
Rock Mass Bulk Modulus of Elasticity, $K$ & $\mathrm{GPa}$ & 25 \\
Minimum Compressive Horizontal Tectonic Stress, $\sigma_{2}$ & $\mathrm{MPa}$ & 37 \\
Linear Thermal Contraction Coefficient of Rock, $\alpha$ & $1 /{ }^{\circ} \mathrm{C}$ & $8 \times 10^{-6}$ \\
\hline
\end{tabular}

\subsection{Initial and Boundary Conditions and Sources for Benchmark Problem 4}

The rock temperature at a depth of $2750 \mathrm{~m}$ is $185^{\circ} \mathrm{C}$. The geothermal gradient in the area is $100^{\circ} \mathrm{C} / \mathrm{km}$ until about a depth of $2300 \mathrm{~m}$ when it falls to $55^{\circ} \mathrm{C} / \mathrm{km}$. Fig. 8-3 shows the vertical temperature profile for the experimental area. 


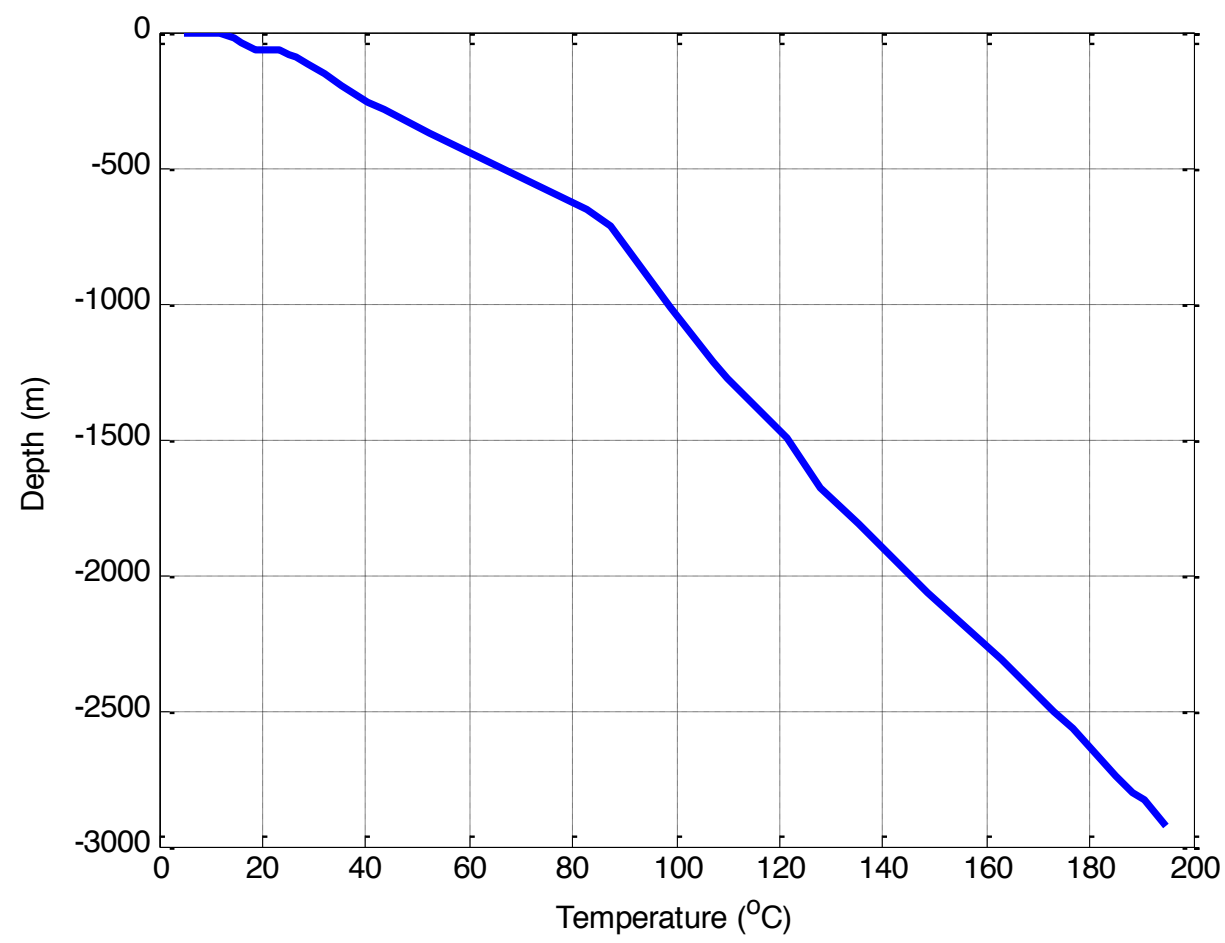

Figure 8-3. Temperature of the rock mass (after (Murphy et al. 1981))

The variation of the injection flow rate and injection pressure during the 75-day test as presented in (Murphy et al. 1981) are shown in Fig. 8-4. It can be seen that the flow rate gradually doubles between days 26 and 36, causing interesting changes and presenting a challenge in making a simple numerical model match. The temperature of the injection water is assumed to be constant at $25^{\circ} \mathrm{C}$ at the surface. The injection temperature at the depth of $2750 \mathrm{~m}$ is elevated, due to heat exchange with the well driven in hot rock. The injection temperature at the entry point of the EGS fracture was not measured and is not given in the reference literature. To overcome this deficiency, the injection temperature at the entry point of the planar fracture is calculated for this benchmark problem using MULTIFLUX, by modeling the coupled, time dependent advection-convection-conduction heat exchange between the coolant fluids in the injection well and the rock mass around it. The calculated injection temperature variation with time is shown in Fig. 8-5 and is used as input in the solution to the EGS fracture model in place of specifying an injection well. 


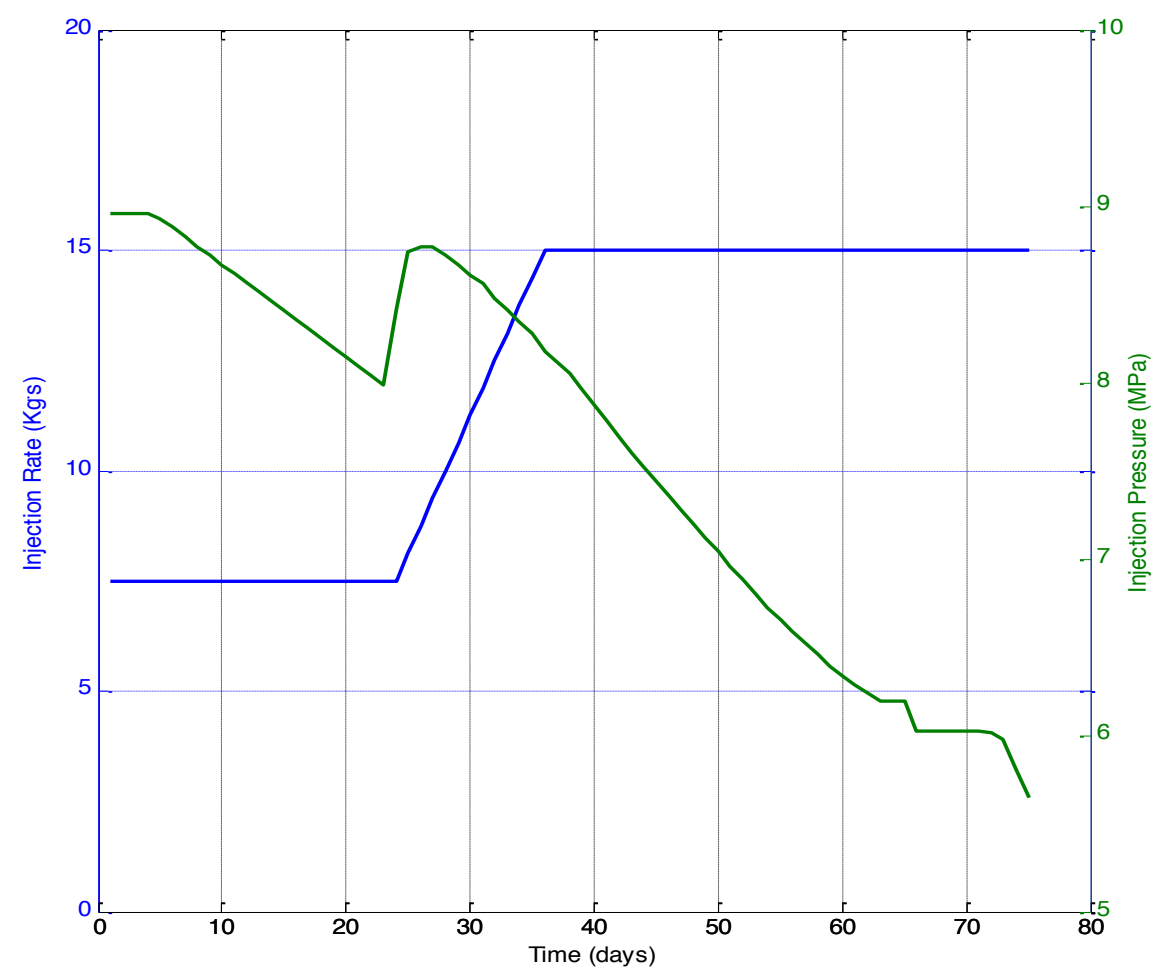

Figure 8-4. Variation of injection pressure and flow rate during a 75-day test of the Phase I reservoir ((Murphy et al. 1981))

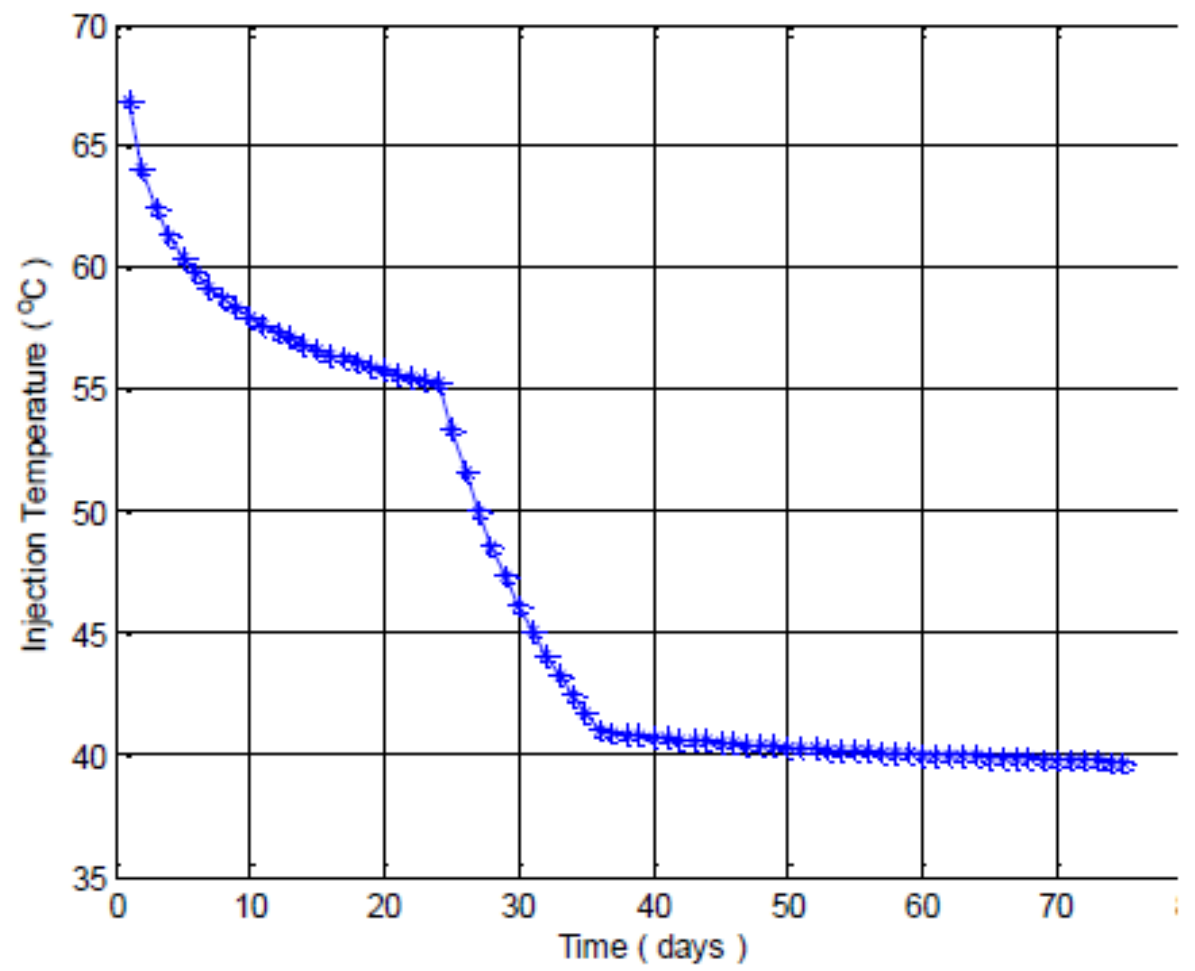

Figure 8-5. Calculated coolant fluid temperature variation with time at the EGS fracture injection point at a depth of $2750 \mathrm{~m}$ 
The production well is modeled to calculate the back-pressure at the extraction point on the EGS fracture at a depth of $2670 \mathrm{~m}$. Since the extraction temperature at the entry point to the well is given from measured data for Fenton Hill, this model-element can be de-coupled. With the coolant flow rate and the input temperature at the extraction point being known, a separate MULTIFLUX model was used to find the total back-pressure at the fracture outlet point at a depth of $2670 \mathrm{~m}$ that includes temperature-varying hydrostatic as well as frictional loss components as a function of time. This back-pressure is shown in Fig. 8-6. The input pressure at the depth of the injection point $(2750 \mathrm{~m})$, depicted in Fig. 8-7, is backcalculated from the specified injection pressure at the surface using the Fenton Hill data by adding the pressure effects (friction loss and hydrostatic) of the injection well from the MULIFLUX model.

For those participant modelers focusing on the EGS fracture only without including the injection and extraction wells, major simplification is provided with the pre-processed functions of the time-dependent (a) injection temperature and pressure (shown in Fig. 8-5 and 8.7, respectively) right at the inlet point of the fracture; as well as (b) the back pressure (shown in Fig. 8-6) given right at the extraction point of the fracture.

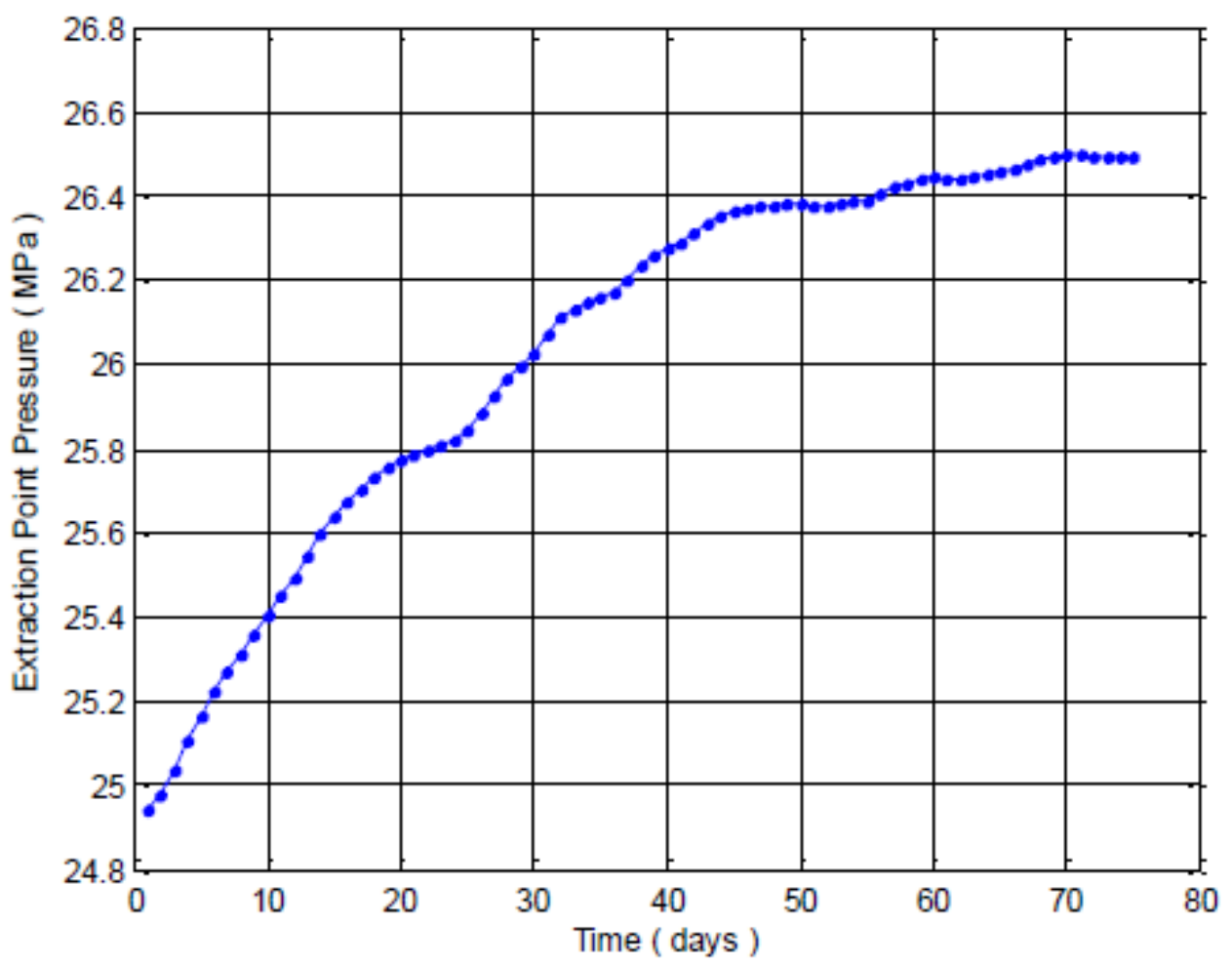

Figure 8-6. Coolant fluid back-pressure variation with time at the EGS fracture extraction point at a depth of $2670 \mathrm{~m}$ 


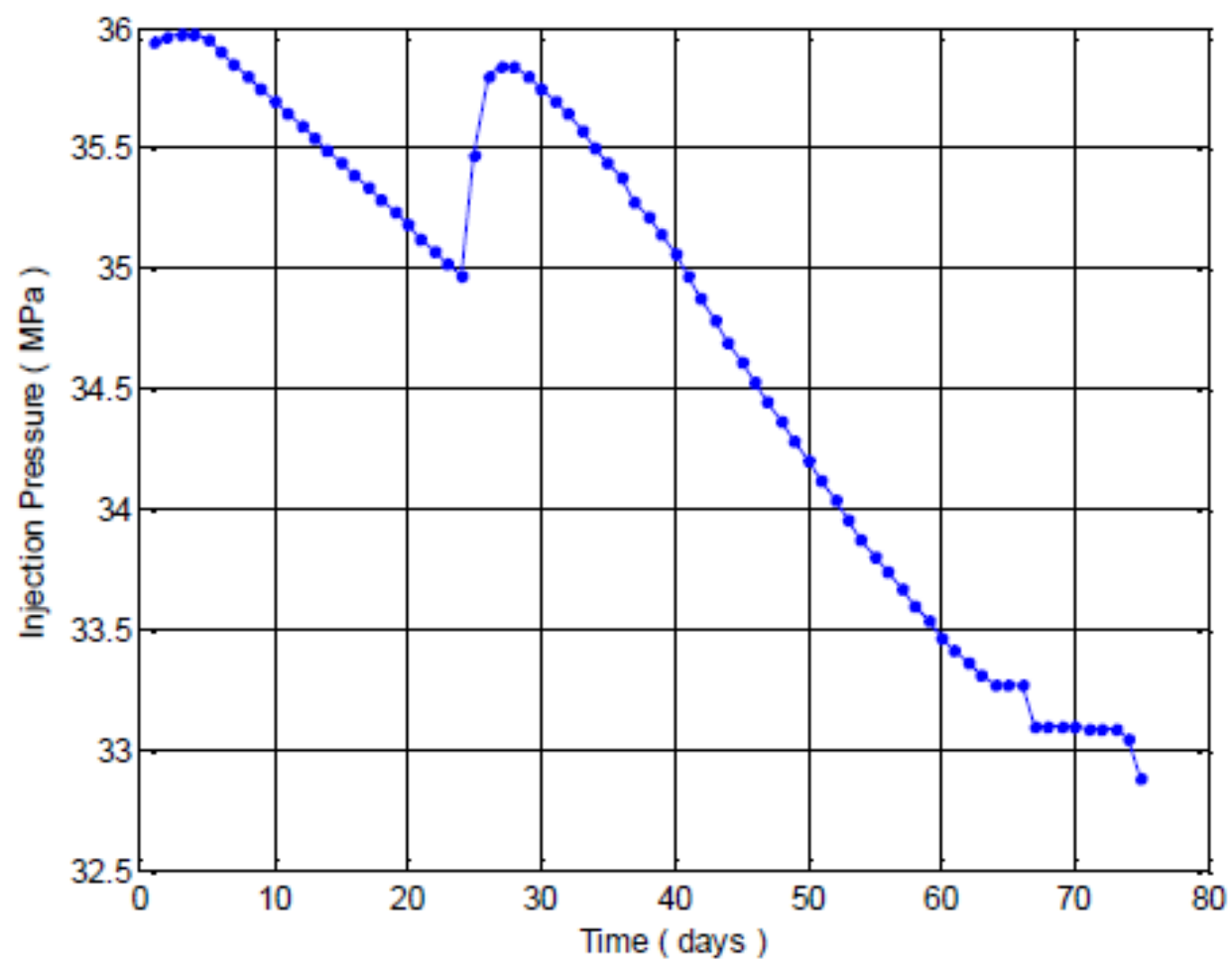

Figure 8-7. Coolant fluid pressure variation at the EGS fracture injection point at a depth of $2750 \mathrm{~m}$

\subsection{Solution Metrics for Benchmark Problem 4}

The expectation of the model output is to match the injection pressure with time for the given experimental flow rate shown in Fig. 8-4, and the extraction temperature shown in Fig. 8-8 for the Fenton Hill experiment. The injection pressure at the surface was used to calculate the injection pressure at a depth of $2750 \mathrm{~m}$ at the fracture plane as a derived metric for those focusing only on the fracture plane model. The post-processed results for the simulated injection pressure at a depth of $2750 \mathrm{~m}$ are shown in Fig. 8-7 as a function of time for 75 days. 


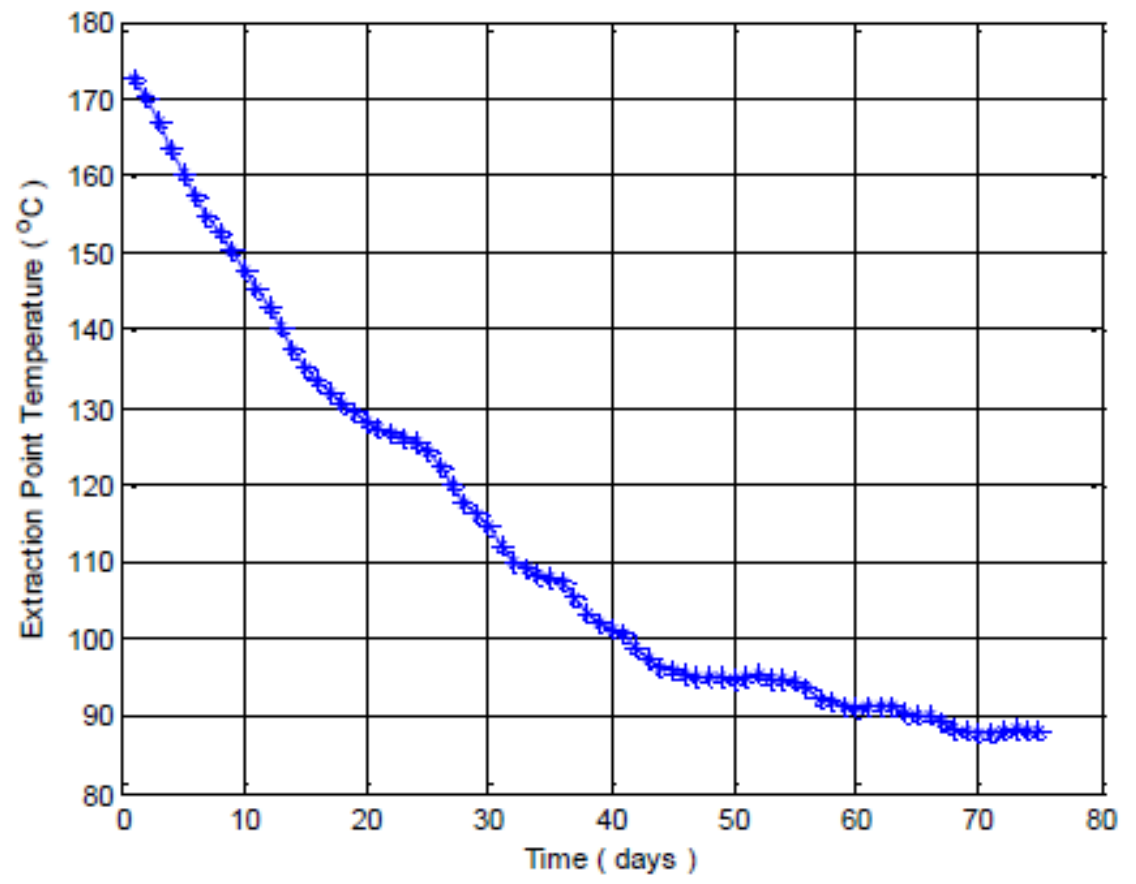

Figure 8-8. Coolant fluid temperature variation at the EGS fracture extraction point at a depth of $2670 \mathrm{~m}$ (from published data for Fenton Hill)

\subsection{Results for Benchmark Problem 4}

The time histories of the simulated injection pressure results at $-2750 \mathrm{~m}$ for the six teams are shown in Fig. 8-9 for Case 1 and Fig. 8-10 for Case 2, respectively. The dashed line in Figs. 8-9 and 8-10 represent the performance metric, post-processed from published measurement results (Murphy et al. 1981). The maximum ISO 13528 uncertainty in the injection pressure is seen to be $\sim 7 \mathrm{MPa}$ for Case 1 (Fig. 8-11) and $\sim 8$ Mpa for Case 2 (Fig. 8-12), about 12\% of the average injection pressure. 


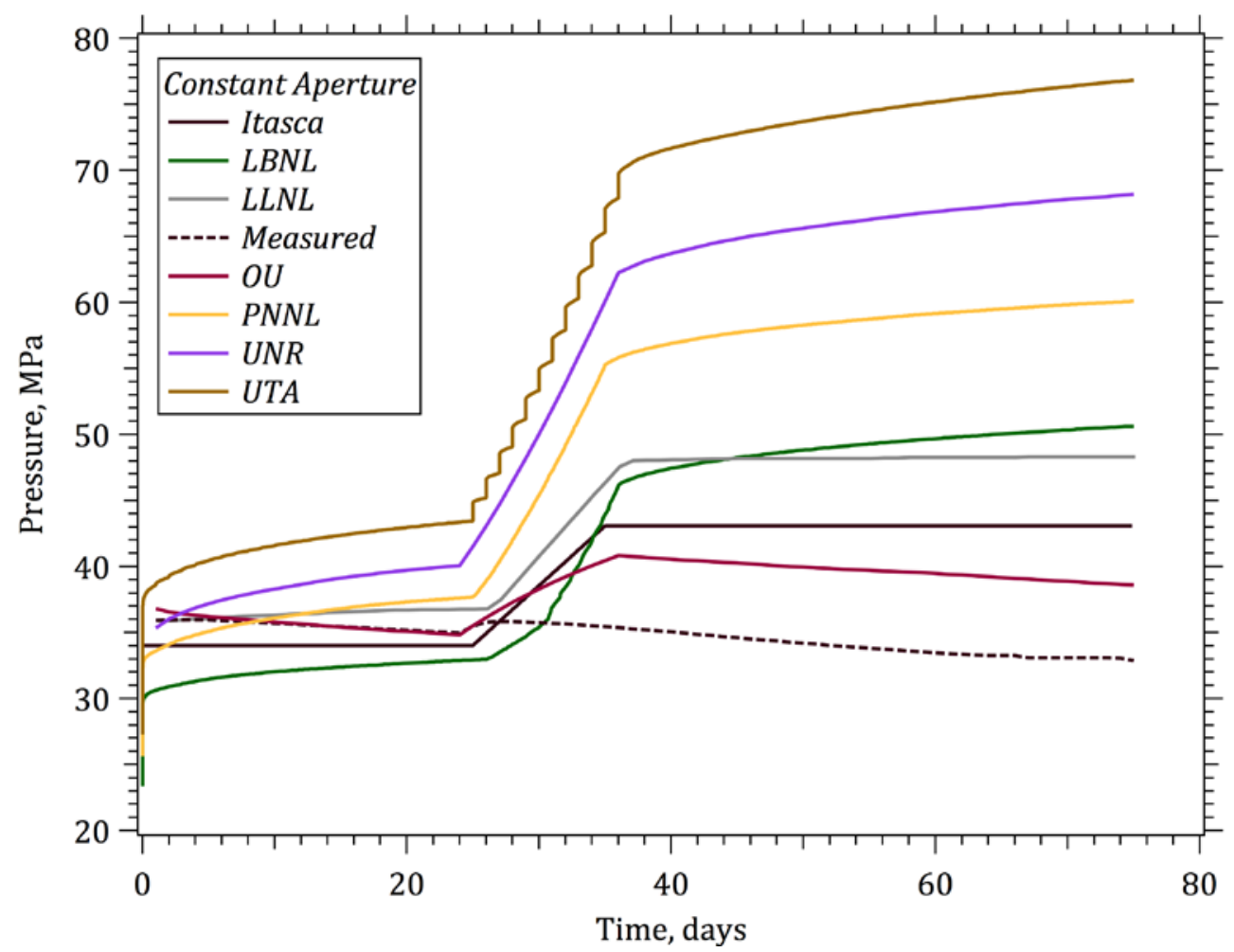

Figure 8-9. Simulation results for injection pressure versus time for Case 1 with constant aperture

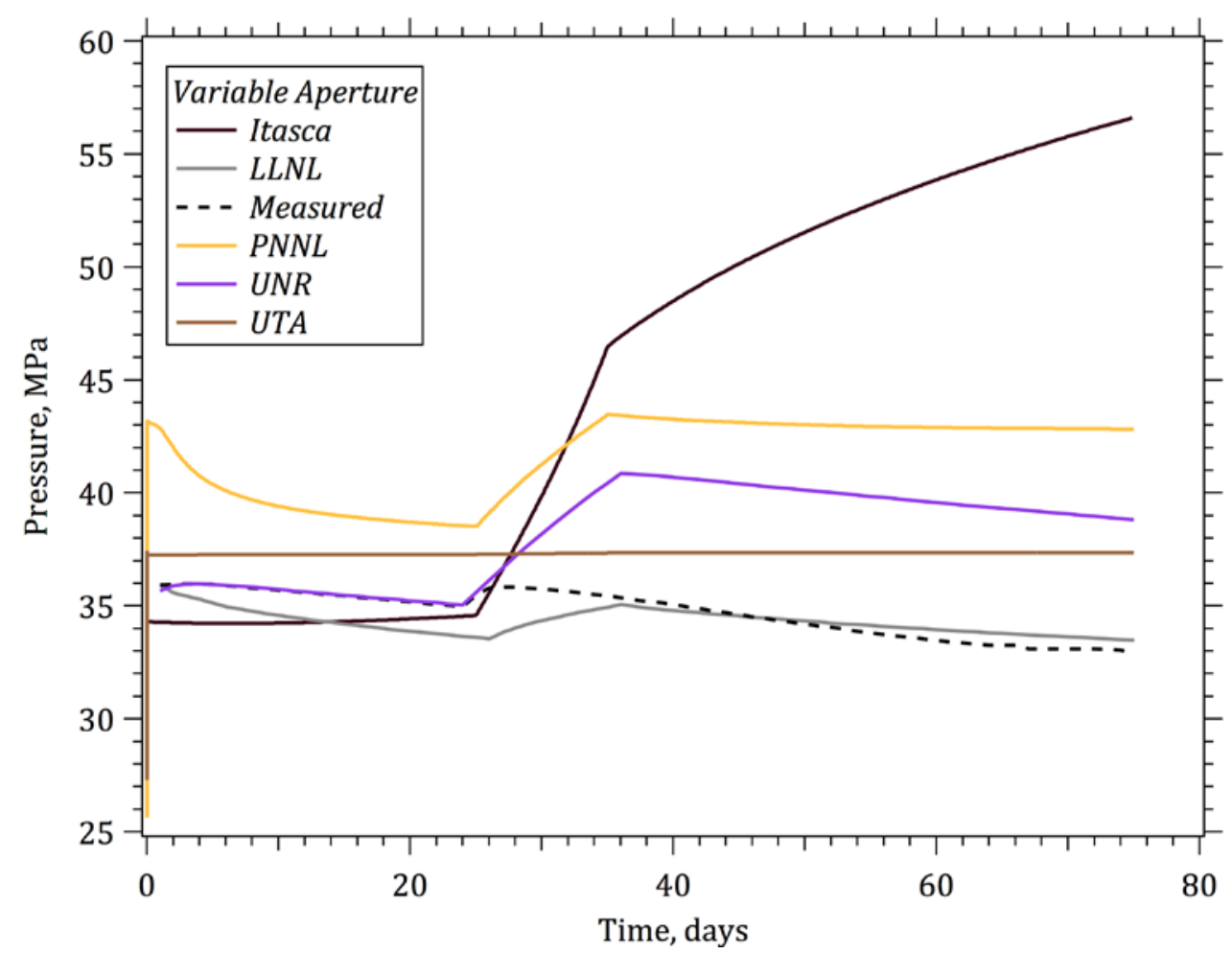

Figure 8-10. Simulation results for injection pressure versus time for Case 2 with variable aperture 


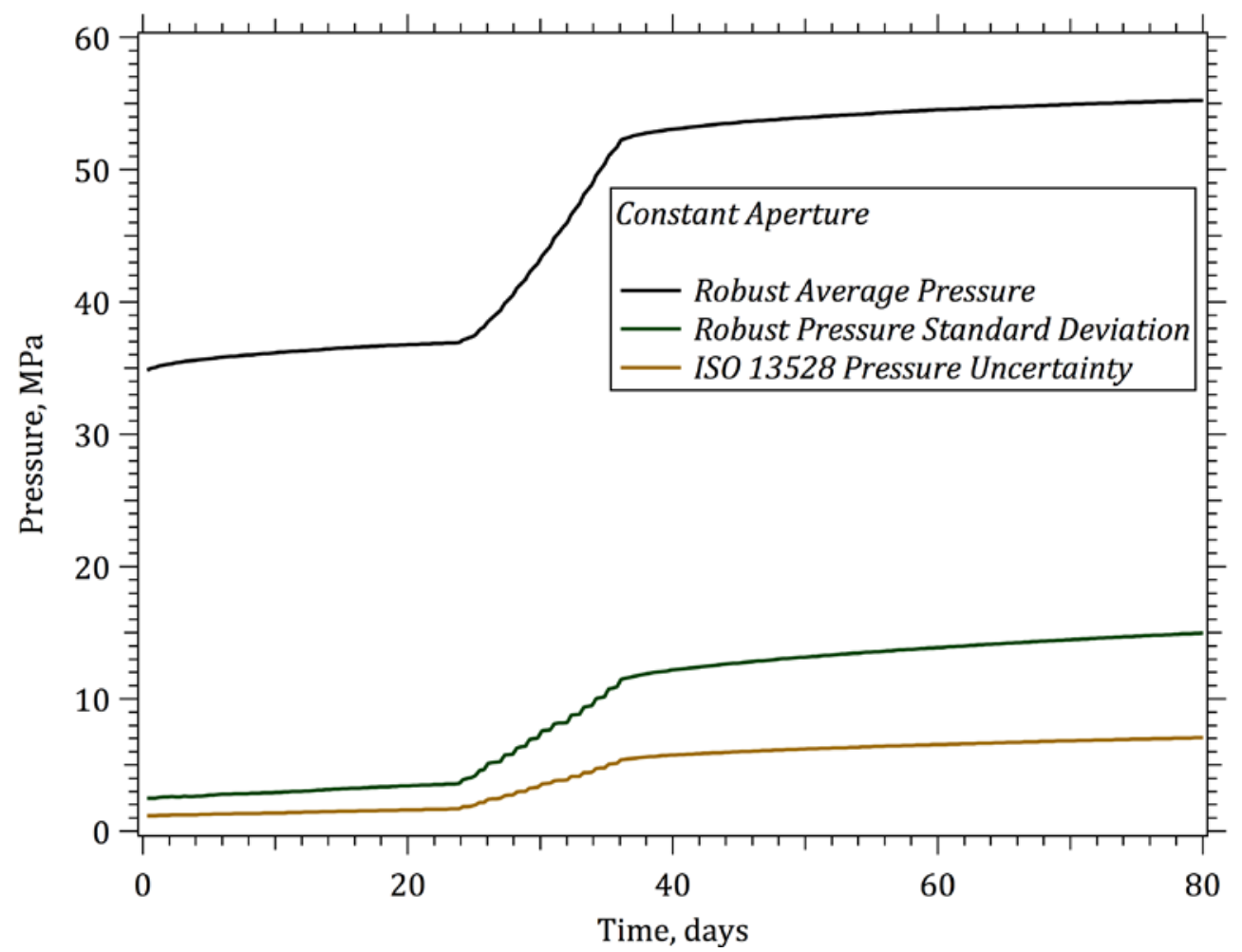

Figure 8-11. ISO 13528 uncertainty for injection pressure versus time for Case 1 with constant aperture

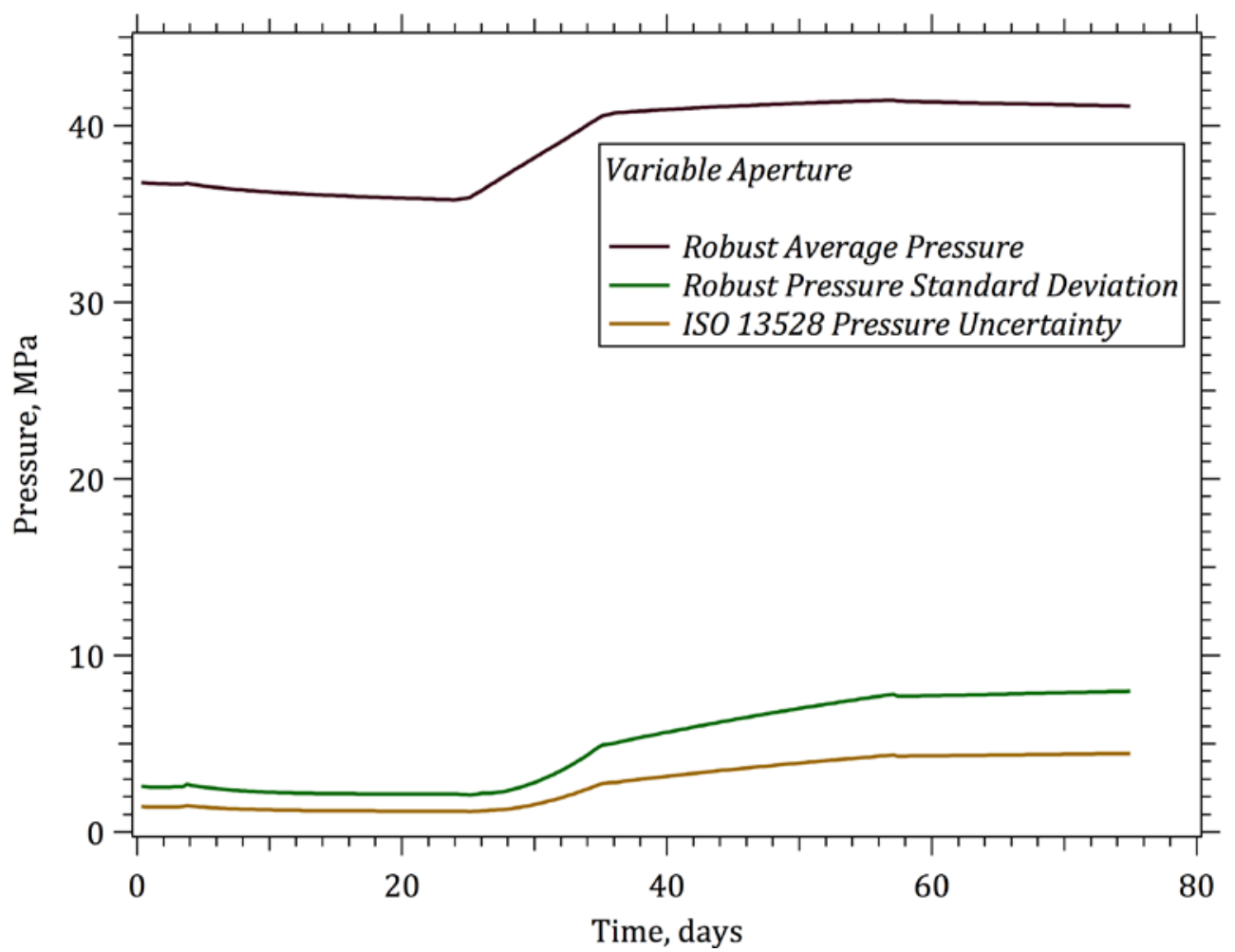

Figure 8-12. ISO 13528 uncertainty for injection pressure versus time for Case 2 with variable aperture 
The limitations of the model in Case 1 are intentional to explore the effects of the temperature-dependent fluid properties and a constant fracture aperture on the shape of the pressure and temperature variations with time. The model results show various degrees of deviations in the curves in Fig 8-9 against the measured pressure function, clustering in two groups. The results of the first group (LBNL, PNNL, UNR and UTA) run nearly parallel to each other and differ only by a vertical shift that is likely due to differences in how a given aperture is converted to permeability of an equivalent poroelastic material. The increase in pressure with time from day 1 to day 24 is caused by the change in the properties of water, most importantly, viscosity with decreasing temperature. This effect is verified by another run, not reported here for brevity, with constant water properties in the same models. The model runs with constant water properties, completed only by a few groups, have shown constant pressure with time for constant fracture aperture. The second group (Itasca and LLNL) shows near-constant pressure with time, caused likely by simplification in the water properties or the thermal model.

The upward trend in pressure change with time for a constant injection flow rate from the constantaperture models is opposite from the measured trend of pressure variation, a proof for fracture aperture opening with time. This opposite trend clearly implies the need for modeling fracture opening due to thermal drawdown through thermo-mechanical coupling. Fracture opening must first compensate for the upward pressure trend due to the viscosity increase with decreasing temperature in addition to further lowering the hydraulic resistance of the fracture. Such an aperture change is modeled in Case 2. The main point to see from these results is that the pressure can perhaps be matched at one point but it will change with time due to temperature variation effects resisting the forced match and demanding a deeper understanding and a refined model.

It is also apparent that the increased flow rate from day 25 will cause a very large pressure increase in a constant-aperture model relative to field data. A question is raised as to why the constant-extension fracture model of the first 24 days cannot match the measured pressure data under a doubled coolant injection flow rate between days 25 through 75 also measured at Fenton Hill and shown as a low-cost computational extension in Fig. 8-9 for Case 1 and Fig. 8-10 for Case 2. The challenge seen from this simplified exercise is to model the aperture change with realistic THM components in order to match reality of the ground at a physical EGS site. The need for a self-opening, thermally-enhanced fracture model is identified, leading to the topic of a future challenge problem.

For the time variation of the injection pressure for Case 2, the results cluster in two groups. The results of the first group (LLNL, PNNL, and UNR) run nearly parallel to each other and differ only by magnitude (vertical shifts) caused by different joint model characteristics: LLNL used an adjusted Barton-Bandis, and PNNL a simpler poroelastic model, while UNR applied a self-propped, linear joint model with constant pressure and temperature coefficients for the fracture. The decrease in pressure with time from day 1 to day 24 agrees well with the measured trend. The second group is represented by the interesting result from UTA with a constant pressure from day 1 through day 24 and onto day 75 as well. The assumption of UTA is that the injection pressure shown in Case 1 for constant aperture goes above the normal stress from some days before day 24 , therefore, the fracture must open as if in the hydro-fracture mode at constant pressure that equals the given normal stress. The injection pressure, however, is higher than that from measurement that runs below the opening pressure for the entire time period; and fails to show the signature of thermal enhancement on pressure variation with time, an important indication of the interplay between the THM components, all present in the Fenton Hill observations.

The time histories of the simulated production temperature results from the six teams are shown in Fig 813 for Case 1 and Figure 8-14 for Case 2, respectively. The dashed line in Figs 8-13 and 8-14 is the performance metric from published measurement results (Murphy et al. 1981). The maximum ISO 13528 uncertainty in the injection temperature is seen to be less than $5^{\circ} \mathrm{C} \mathrm{MPa} \mathrm{for} \mathrm{both} \mathrm{Case} 1$ (Fig. 8-25) and Case 2 (Fig. 8-26), about 12\% of the average injection pressure. 


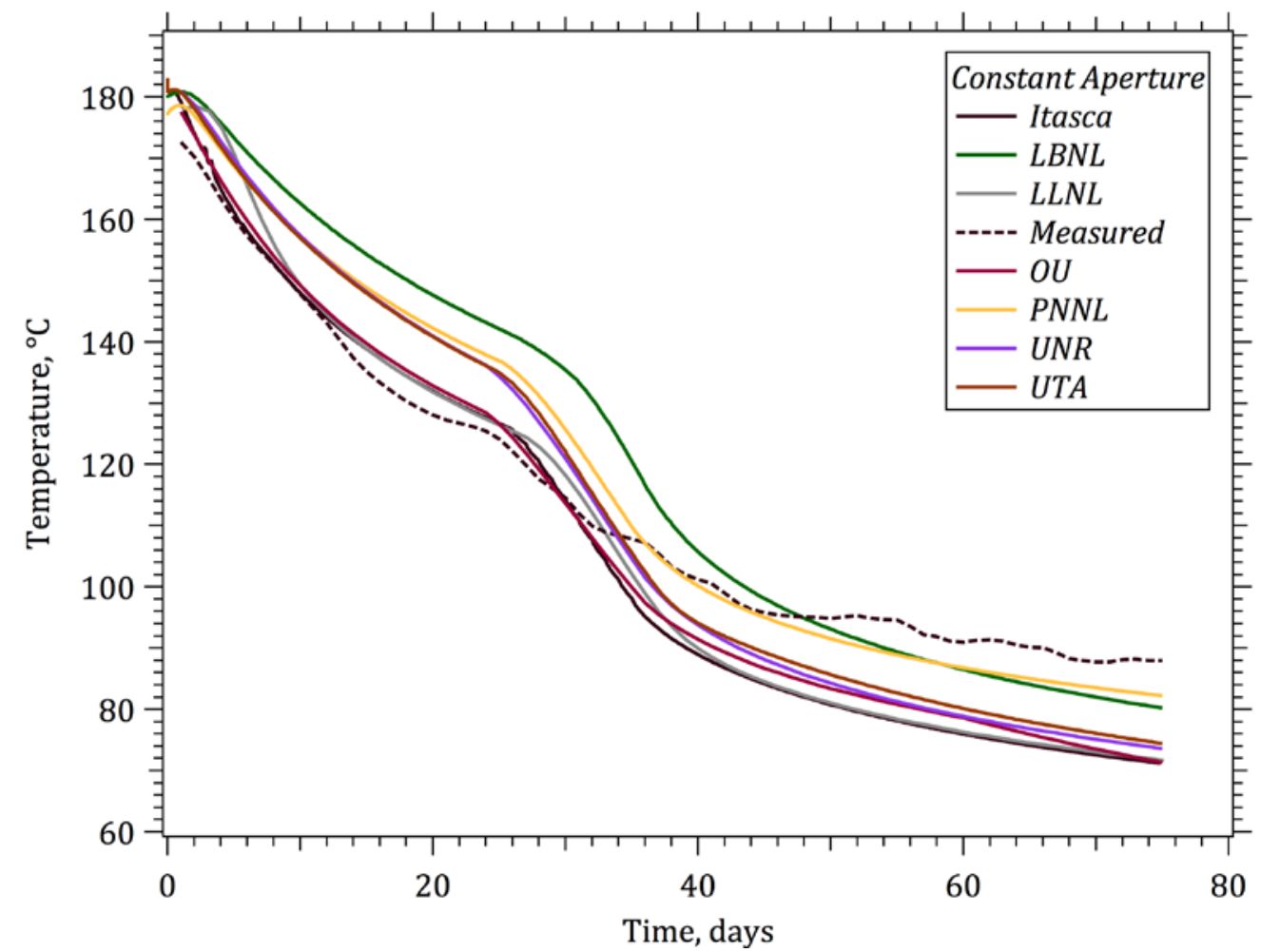

Figure 8-13. Simulation results for temperature versus time for Case 1 with constant aperture

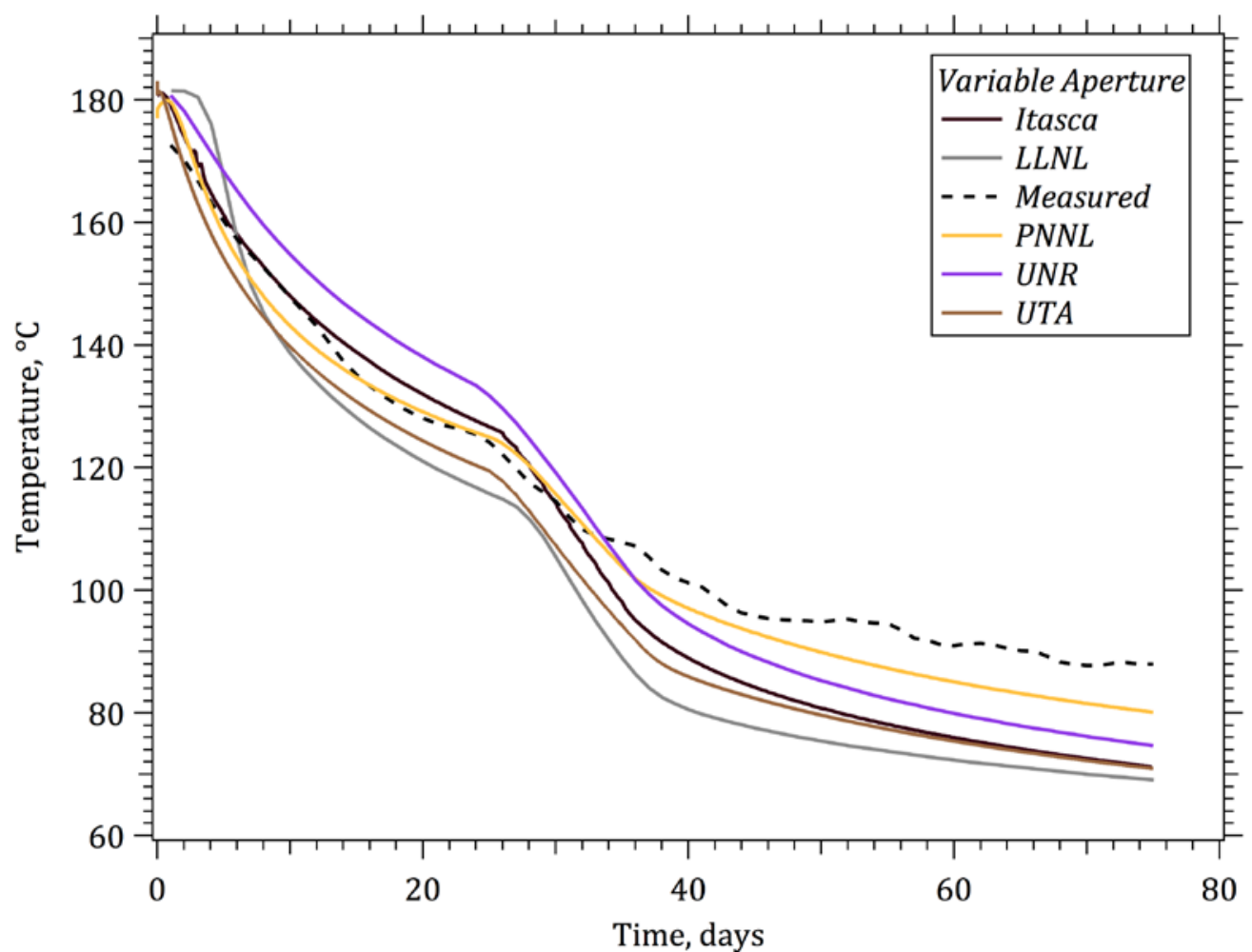

Figure 8-14. Simulation results for temperature versus time for Case 2 with variable aperture 


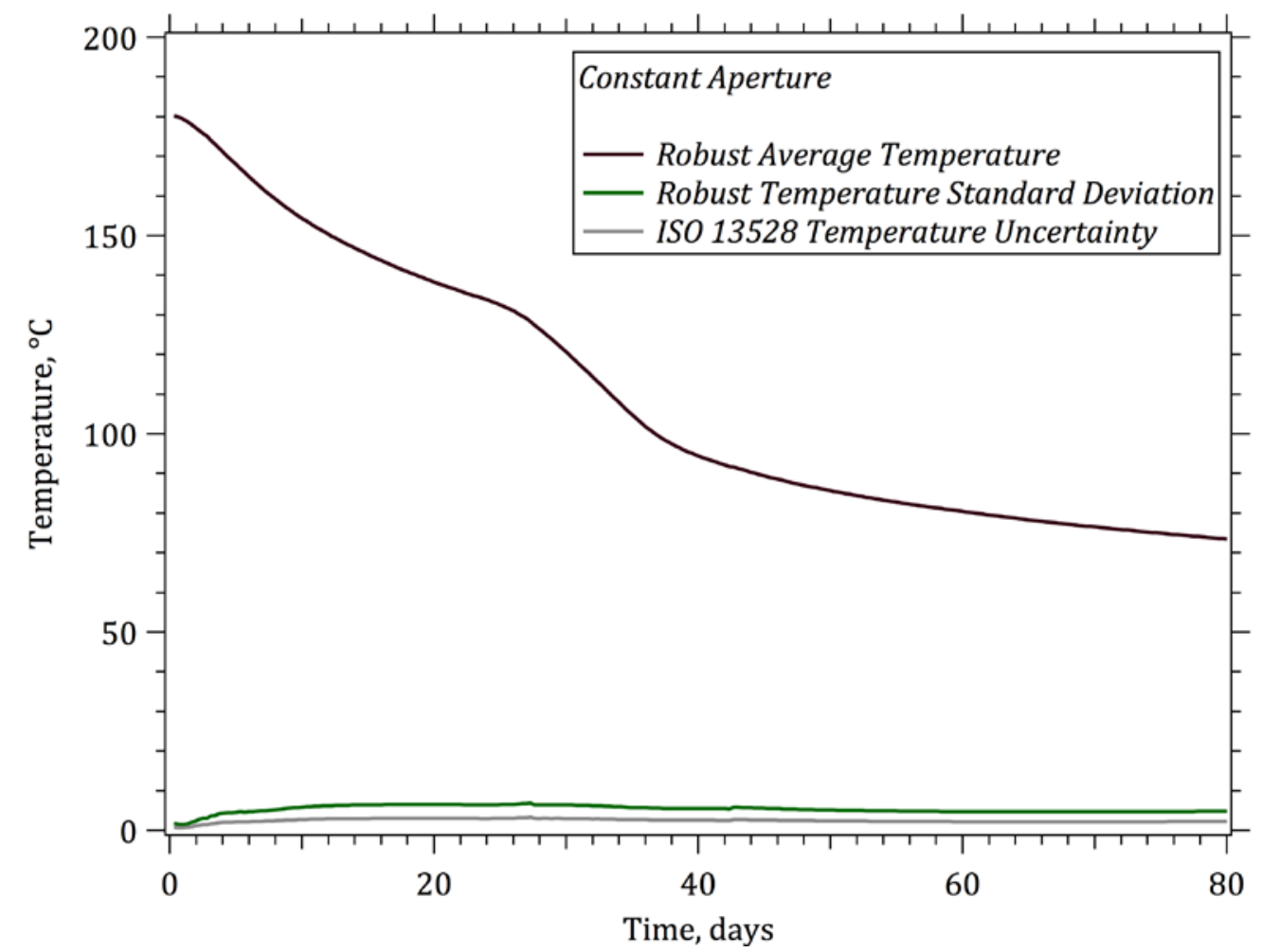

Figure 8-15. ISO 13528 uncertainty for temperature versus time for Case 1 with constant aperture

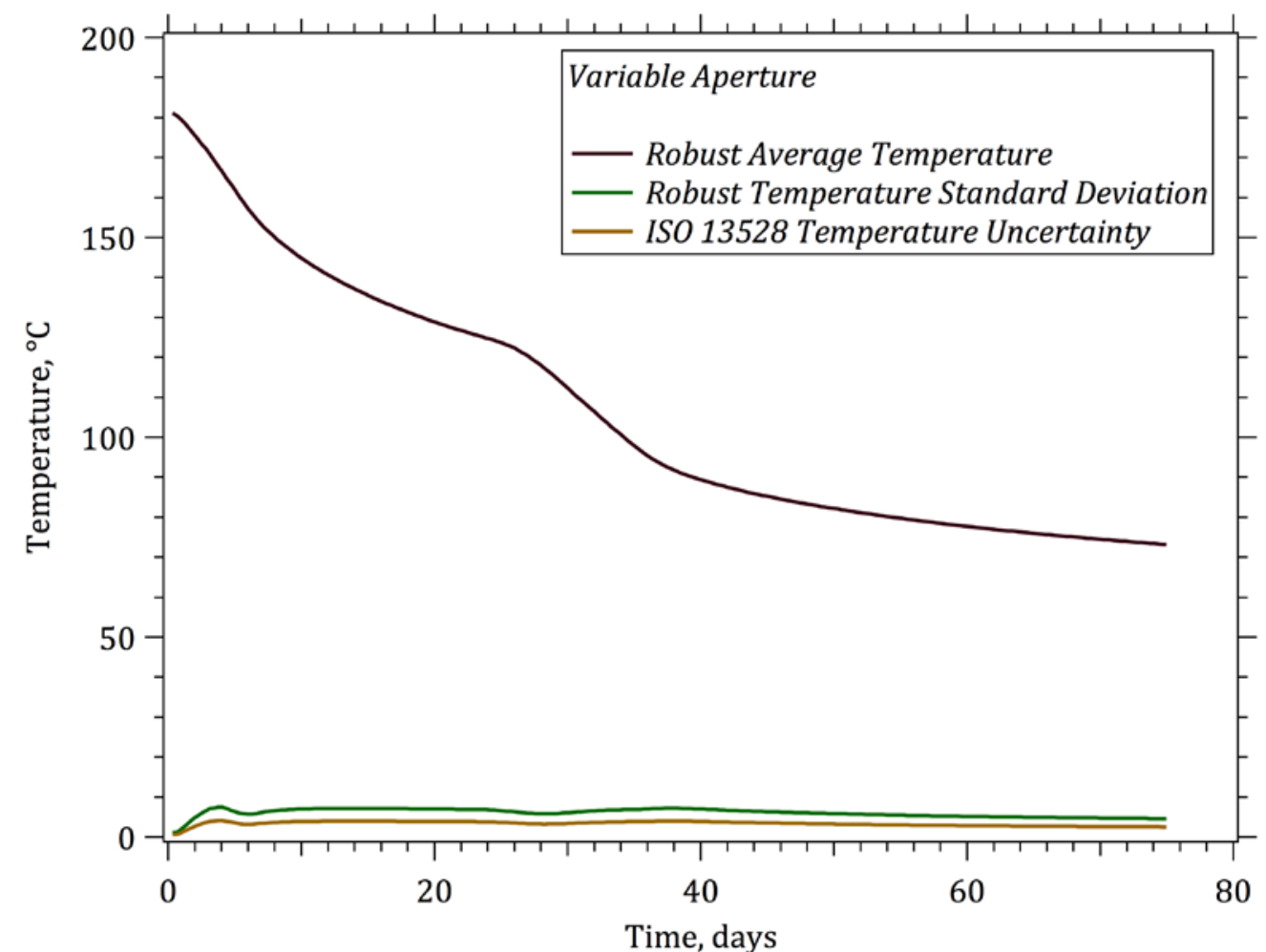

Figure 8-16. ISO 13528 uncertainty for temperature versus time for Case 2 with variable aperture 
Results for the first 24 days are considered as the primary, quantitative performance criteria. Differences in the measured trends for the rest of the time period serve for conclusions toward model limitations and the challenges that EGS models must face in matching field data.

For the time variation of the production temperature for Case 1, the results cluster in two groups. The results of the first group (LBNL, PNNL, UNR, and UTA) run very close to each other albeit LBNL's is a bit of an outlier but on the same trend. All models over-predict the extraction temperature significantly and thus the available heat extraction potential; and all under-predict the thermal drawdown. The results of this group are also in very good agreement with a previous, independent simulation result conducted at LBNL as part of a funded research project (Danko and Bahrami 2013a) and posted at GTO-CCS' Velo site as a target metric for comparison. The difference in over-prediction is explained by the penny-shaped, constant fracture aperture with a more efficient fluid delivery to the edge area which is not likely to be the Case in a lens-shaped fracture representative to the openings in the field. Indeed, if the lens-shape form is enforced to the fracture of the same diameter, the thermal drawdown matches the measured temperature variation very closely (Danko and Bahrami 2013a). The second group (ITASCA, LLNL) shows very good agreement with the measured data likely driven by a less effective heat exchange process in or around the fracture between the coolant fluid and the host rock.

For the time variation of the production temperature for Case 2, the results also cluster in two groups. The first group (LLNL, PNNL, and UT Austin) run very close to each other and match the target metric excellently for the first 24 days. The UNR solution in the second group shows only a very slight improvement over the Case 1 result for temperature for this time period. This is not surprising, since the fracture, essentially penny-shape, is not much different from Case 1 to Case 2, since the fracture model lacks the three-dimensional stress-field effects. The refinement for three-dimensional effects has not been assigned to this problem though it has been implemented with some success (Danko and Bahrami 2013b).

\subsection{Discussion of Benchmark Problem 4 Results}

Various approaches are used in different models regarding the responses of a single planar fracture in the rock based on the first experimental EGS in the U.S. at Fenton Hill, Phase I. The rock mechanics component in all models except for that of UTA includes the thermo-elastic response of the fracture layer coupled to the thermal model of the reservoir during coolant injection.

Only the first 24-day injection and production period is considered in the effort of matching the simulation results of a selected THM model with experimental data. The working hypothesis is a good match for the variations both for injection pressure and thermal drawdown for 24 days. Subsequent to the 24-day time period, the injection rate is gradually doubled according to the field experiment. The selected model, if linear, is expected to be poor in matching both pressure and temperature variations against field measurements.

Various model assumptions and successes have been shown for the solutions to Benchmark Problem 4. Some models used more advance model assumptions than what was prescribed to test. For example, LLNL used a Barton-Bandis joint model with calibrated parameters and obtained a much better match than others, a courageous attempt to go beyond the stated purpose of Case 2 in order to point out the failure of the simple model assumption. However, even the LLNL model fails to match the metric for the production temperature variation which points to an increasing fracture diameter with time and injection flow rate and an increase of the active heat transfer surface area of the EGS fracture at Fenton Hill. All models significantly over-predict the thermal drawdown for Fenton Hill between the first 24-day and the second 51-day time periods. This is one of the differences that calls for further investigation of model 
capabilities and necessary model-refinements to support advanced EGS studies, and will be addresses in the GTO-CCS Challenge Problems. 



\title{
9.0 Benchmark Problem 5: Amorphous Silica Dissolution/Precipitation In A Fracture Zone
}

\author{
Problem Champion: Mark White, Pacific Northwest National Laboratory
}

Benchmark Problem 5 considered the flow of water through an idealized horizontal fracture with temperature dependent reaction of the water with amorphous silica, the formation mineral. This problem is an altered version of the simulations conducted by (Xu and Pruess 2004) that investigated the effects of mineral scaling and clay swelling in a fractured geothermal reservoir. The chemical reaction network for this problem was reduced from the (Xu and Pruess 2004) configuration to a single dissolving mineral, yielding a network of three reactions:

$$
\begin{aligned}
& \mathrm{SiO}_{2}(\mathrm{am}) \leftrightarrow \mathrm{SiO}_{2}(\mathrm{aq}) \text { kinetic dissolution } \\
& \mathrm{SiO}_{2}(\mathrm{aq}) \leftrightarrow \mathrm{H}^{+}+\mathrm{HSiO}_{3}^{-} \text {aqueous equilibrium } \\
& \mathrm{H}_{2} \mathrm{O} \leftrightarrow \mathrm{H}^{+}+\mathrm{OH}^{-} \text {aqueous equilibrium }
\end{aligned}
$$

Although the number of chemical species was reduced, the problem retained the challenges of having the kinetic amorphous silica dissolution reaction having temperature dependence in both the kinetic rate and equilibrium constants, following the general form of the rate law (Steefel and Lasaga 1994). The fracture zone comprises three regions: 1) fracture, 2) altered granite, and 3) unaltered granite and the volume fractions of amorphous silica, inert minerals, and pore space differ across the rock regions. The problem is driven by the injection of water into the fracture, thereafter the flow is predominately through the fracture, but the altered granite and unaltered granite have finite intrinsic permeability and porosity. The two variants on the problem are either the injection of "pure" water or the injection of "recycled" water. Under both scenarios, water at $65^{\circ} \mathrm{C}$ is injected into the fracture zone, which is initially at $200^{\circ} \mathrm{C}$. The dissolution and precipitation of amorphous silica along the length of the fracture zone is to be computed for two forms of injected water: 1) "pure" water and 2) "recycled" water. The "recycled" water scenario assumes a dissolved composition which is supersaturated with respect to amorphous silica, the only mineral considered in this analysis. Changes in intrinsic permeability and porosity due to mineral dissolution and precipitation were also considered. A relationship between changes in porosity and intrinsic permeability developed by (Verma and Pruess 1988) is used to more accurately capture the effect of pore-throat clogging by precipitates. We anticipate that some aspects of this problem will yield results that are dependent on the spatial and potentially temporal discretizations chosen. Three teams participated in the solutions using various simplifying assumptions, multiple runs and THC models as listed in Table $9-1$.

Table 9-1. Participating Teams and Simulators Used in the Solution of Benchmark Problem 5

\begin{tabular}{lll}
\hline Simulation Team & Team Identifier & Code(s) \\
\hline Lawrence Berkeley National Laboratory & LBNL & TOUGHREAC \\
Lawrence Livermore National Laboratory & LLNL & NUFT \\
Pacific Northwest National Laboratory & PNNL & STOMP \\
\hline
\end{tabular}

\subsection{Geometry and Input Data for Benchmark Problem 5}

An idealized vertical fracture zone is modeled, with a fracture half-width of $0.026 \mathrm{~m}$. The fracture zone is adjacent to an altered granite zone with a width of $0.2 \mathrm{~m}$. Adjacent to the altered granite zone is an unaltered granite zone that extends to $120 \mathrm{~m}$. The fracture is assumed to be $150 \mathrm{~m}$ in length. A unit 
length of $1.0 \mathrm{~m}$ of the vertical extent of the fracture is considered, creating a two-dimensional problem as shown in Fig. 9-1. For the "pure" water scenario, water with $\mathrm{pH}$ of 7.0 without dissolved $\mathrm{SiO}_{2}(\mathrm{aq})$ and $\mathrm{HSiO}_{3}-$ at $65^{\circ} \mathrm{C}$ is injected at one end of the horizontal column and is produced at the opposite end. For the "recycled" water scenario, the composition of the water being produced from the outlet of the fracture after 10 years is to be re-injected. Cooling of the "recycled" water from the outlet to $65^{\circ} \mathrm{C}$ is assumed to occur without precipitation of $\mathrm{SiO}_{2}(\mathrm{aq})$. Water reacts with the surfaces of the fracture, altered granite and unaltered granite. Hydrologic and thermal properties for the three rock zones are shown in Table 9-2.

Unaltered Granite

Altered Granite

Fracture

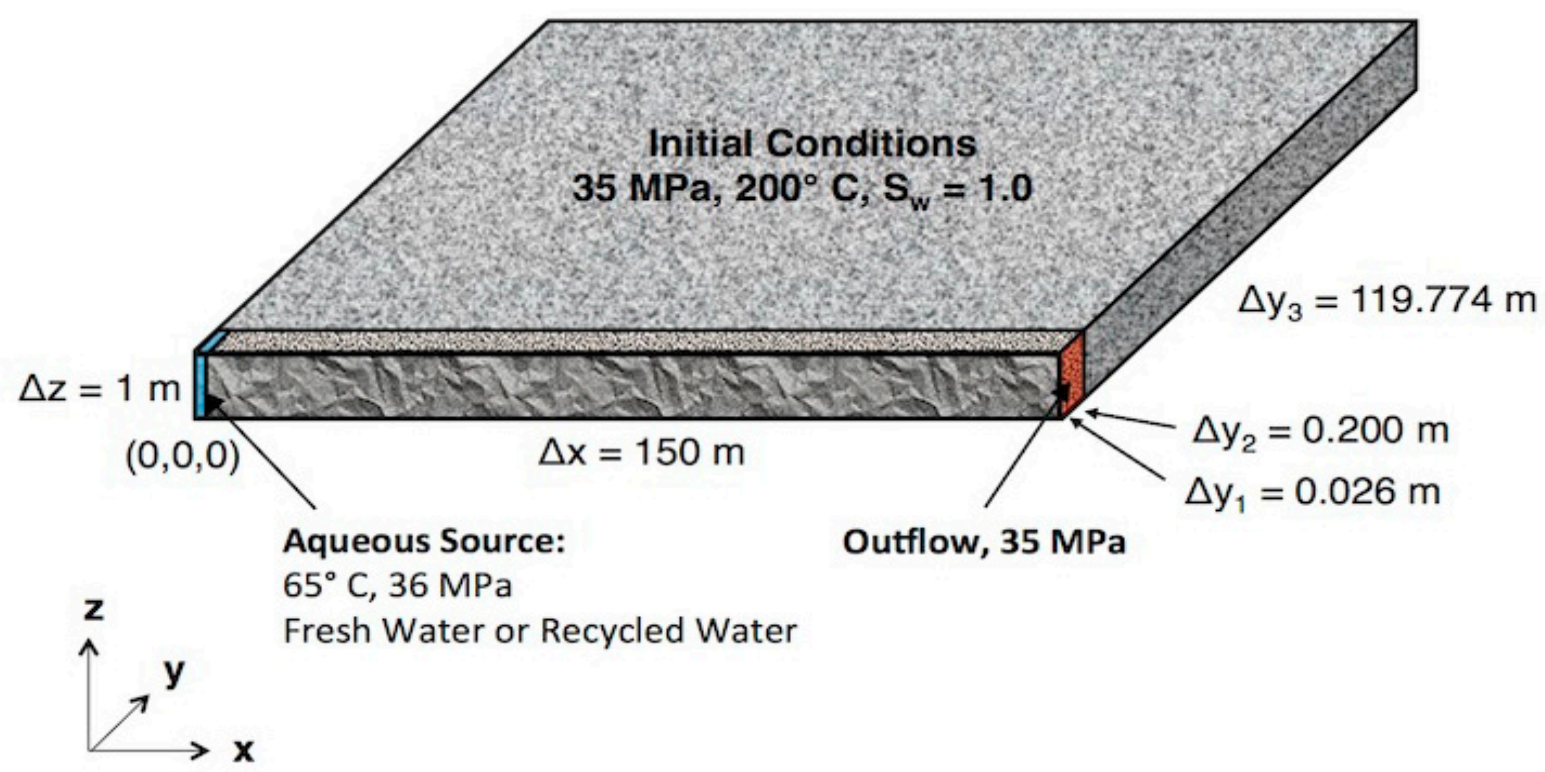

Not to scale

Figure 9-1. Idealized fracture, altered granite, and unaltered granite zones

Table 9-2. Material Property Table

\begin{tabular}{lllll}
\hline Property & Units & Fracture & $\begin{array}{l}\text { Altered } \\
\text { Granite }\end{array}$ & $\begin{array}{l}\text { Unaltered } \\
\text { Granite }\end{array}$ \\
\hline Intrinsic Permeability, $k$ & $\mathrm{~m}^{2}$ & $2.0 \times 10^{-12}$ & $2.0 \times 10^{-15}$ & $2.0 \times 10^{-18}$ \\
Porosity, $\phi$ & -- & 0.2 & 0.1 & 0.02 \\
Thermal Conductivity, $\kappa$ & $\mathrm{W} / \mathrm{m} \mathrm{K}$ & 2.9 & 3.0 & 3.0 \\
Tortuosity Factor, $\tau$ & -- & 0.3 & 0.1 & 0.05 \\
Grain Density, $\rho_{g}$ & $\mathrm{~kg} / \mathrm{m}^{3}$ & 2650 & 2650 & 2650 \\
Grain Specific Heat, $c_{g}$ & $\mathrm{~J} / \mathrm{kg} \mathrm{K}^{2}$ & 1000 & 1000 & 1000 \\
Aqueous Diffusion Coefficient, $D$ & $\mathrm{~m}^{2} / \mathrm{s}$ & $1 \times 10^{-9}$ & $1 \times 10^{-9}$ & $1 \times 10^{-9}$ \\
\hline
\end{tabular}




\subsection{Initial and Boundary Conditions and Sources for Benchmark Problem 5}

The initial reservoir temperature and pressure were $200^{\circ} \mathrm{C}$ and $35 \mathrm{MPa}$, respectively. The initial mineralogical composition of the three zones is shown in Table 9-3. The initial water chemical compositions for the three rock zones will be that which is in equilibrium with the corresponding mineral composition at $200^{\circ} \mathrm{C}$. An overpressure of $1 \mathrm{MPa}$ was applied to the injection end of the horizontal column. Water was injected at $65^{\circ} \mathrm{C}$ and $36 \mathrm{MPa}$ over the fracture and altered granite zones. The production end of the column was maintained at $35 \mathrm{MPa}$, over the fracture and granite zones. All surfaces beyond those used for injection and production were considered to be adiabatic. Two scenarios for water injection were considered: 1) "pure" water and 2) "recycled" water.

Table 9-3. Initial mineralogical volume fractions of rock zones

\begin{tabular}{llll}
\hline Mineral & Fracture & $\begin{array}{l}\text { Altered } \\
\text { Granite }\end{array}$ & $\begin{array}{l}\text { Unaltered } \\
\text { Granite }\end{array}$ \\
\hline $\mathrm{SiO}_{2}(\mathrm{am})$ & 0.35 & 0.33 & 0.24 \\
Nonreactive Minerals & 0.45 & 0.57 & 0.74 \\
Porosity & 0.20 & 0.10 & 0.02 \\
\hline
\end{tabular}

\subsection{Constitutive Relationships for Benchmark Problem 5}

The (Verma and Pruess 1988) model was used to relate changes in porosity with changes in intrinsic permeability; where, the critical porosity was defined as $80 \%$ of the initial porosity and the functional exponent was 2:

$$
\frac{k}{k_{o}}=\left(\frac{\phi-\phi_{c}}{\phi_{o}-\phi_{c}}\right)^{n} ; \phi_{c}=0.8 \phi_{o} ; n=2
$$

where, $k$ is the intrinsic permeability, $\mathrm{m}^{2}, k_{o}$ is the reference intrinsic permeability, $\mathrm{m}^{2}, \phi$ is the porosity, $\phi_{c}$ is the critical porosity, $\phi_{o}$ is the reference porosity, and $n$ is the functional exponent.

\subsection{Reaction Network for Problem 5}

As shown in Eqn. (6), the reaction network comprises one kinetic reaction for the dissolution of $\mathrm{SiO}_{2}$ and two equilibrium reactions between the aqueous species. For the equilibrium reaction the equilibrium constants were assumed to be a function of temperature:

$$
\log K=c_{1} \ln (T)+c_{2}+c_{3} T+\frac{c_{4}}{T}+\frac{c_{5}}{T^{2}} ; \text { with } \operatorname{Tin}^{\circ} \mathrm{K}
$$

where, the coefficients for the two equilibrium reactions are shown in Table 9-4. The kinetic reaction rate is expressed in terms of the change in amorphous silica per time, mol/s, using a common form for kinetic mineral dissolution and precipitation (Steefel and Lasaga 1994): 


$$
\frac{\partial \mathrm{SiO}_{2}(\mathrm{am})}{\partial t}=k_{25} A_{m}\left[1-\frac{Q_{m}}{K_{m}}\right] \exp \left[\frac{-E_{a}}{R}\left(\frac{1}{T}-\frac{1}{T_{25}}\right)\right] ; \text { with } T \text { in }^{\circ} \mathrm{K}
$$

where, $\mathrm{SiO}_{2}(\mathrm{am})$ are the moles of amorphous silica, $k_{25}$ is the reaction rate at $25^{\circ} \mathrm{C}, \mathrm{mol} / \mathrm{m}^{2} \mathrm{~s}, A_{m}$ is the specific mineral surface area, $\mathrm{cm}^{2} / \mathrm{gm}, Q_{m}$ is the ion activity product, $K_{m}$ is the equilibrium constant, $E_{a}$ is the activation energy, $\mathrm{kJ} / \mathrm{mol}, R$ is the ideal gas constant $(8.314 \mathrm{~J} / \mathrm{K} \mathrm{mol}), T$ is the temperature, ${ }^{\circ} \mathrm{K}$ and $T_{25}$ is the reference temperature $\left(298.15^{\circ} \mathrm{K}\right)$. The parameters for this equation (Eqn. (9.4)) are shown in Table 9-5.

Table 9-4. Equilibrium reactions and coefficients for the temperature-dependent equilibrium constant

\begin{tabular}{llllll}
\hline Equation & $c_{1}$ & $c_{2}$ & $c_{3}$ & $c_{4}$ & $c_{5}$ \\
\hline $\mathrm{H}_{2} \mathrm{O} \leftrightarrow \mathrm{H}^{+}+\mathrm{OH}$ & $1.167 \times 10^{2}$ & $-7.455 \times 10^{2}$ & $-1.170 \times 10^{-1}$ & $3.916 \times 10^{4}$ & $-2.637 \times 10^{6}$ \\
$\mathrm{SiO}_{2}(\mathrm{aq}) \leftrightarrow \mathrm{H}^{+}+\mathrm{HSiO}_{3}^{-}$ & 5.733 & $-1.374 \times 10^{1}$ & $-3.538 \times 10^{-2}$ & $-8.173 \times 10^{3}$ & $8.088 \times 10^{5}$ \\
\hline
\end{tabular}

Table 9-5. Kinetic reactions, parameters, and coefficients for the temperature-dependent equilibrium constant

\begin{tabular}{llllllll}
\hline $\begin{array}{l}k_{25} \\
\left(\mathrm{~mol} / \mathrm{m}^{2} \mathrm{~s}\right)\end{array}$ & $\begin{array}{l}E_{a} \\
(\mathrm{~kJ} / \mathrm{mol})\end{array}$ & $\begin{array}{l}A_{m} \\
\left(\mathrm{~cm}^{2} / \mathrm{gm}\right)\end{array}$ & $c_{1}$ & $c_{2}$ & $c_{3}$ & $c_{4}$ & $c_{5}$ \\
\hline $3.8 \times 10^{-10}$ & 49.8 & 9.8 & $1.014 \times 10^{2}$ & $-6.658 \times 10^{2}$ & $-7.844 \times 10^{-2}$ & $4.266 \times 10^{4}$ & $-3.055 \times 10^{6}$ \\
\hline
\end{tabular}

The kinetic reaction rate, shown in Eqn. (9.4), depends on the ratio of the ion activity product to the equilibrium product. The greater the difference in these two parameters the greater the departure from equilibrium and the faster the reaction rate. For a general kinetic reaction where species $A$ and $B$ react to produce $C$ and $D$ :

$$
a A+b B \Leftrightarrow c C+d D
$$

where $a, b, c$, and $d$ represent the number of moles of these constituents. At chemical equilibrium, the distribution of chemical species mass between reactants and products can be expressed as:

$$
K_{m}=\frac{\left[C_{e q}\right]^{c}\left[D_{e q}\right]^{d}}{\left[A_{e q}\right]^{a}\left[B_{e q}\right]^{b}}
$$

where $[A],[B],[\mathrm{C}]$, and $[D]$ are the activities for the reactants and products at equilibrium. The ion activity product is computed in the same fashion as the equilibrium constant:

$$
Q_{m}=\frac{[C]^{c}[D]^{d}}{[A]^{a}[B]^{b}}
$$

If $Q_{m} / K_{m}>1$, then the reaction tends toward the reactants (precipitation of $\mathrm{SiO}_{2}(\mathrm{am})$ for Eqn. (9.4)), and conversely if $Q_{m} / K_{m}<1$ then the reaction tends toward the products (dissolution of $\mathrm{SiO}_{2}(\mathrm{am}$ ) for Eqn. (9.4)). 
Species activity is computed from the product of the species concentration times its activity coefficient. Activity coefficients were calculated using the B-dot model, which is an extension of the Debye-Huckel model, with temperature dependent coefficients. There are two equations for computing the activity coefficient as a function of temperature and ionic strength, one for charged species and a second for neutral or nonpolar species. As the kinetic reaction for this problem only involves the dissolution of amorphous silica, without changes in associated charge, the second form of the equation is used:

$$
\begin{array}{lll}
\log \gamma_{o}=a I+b I^{2}+c I^{3} & \\
a=\sum_{i=0}^{3} c_{a}^{i} T^{i}\left({ }^{\circ} \mathrm{C}\right) & b=\sum_{i=0}^{4} c_{b}^{i} T^{i}\left({ }^{\circ} \mathrm{C}\right) & c=\sum_{i=0}^{4} c_{c}^{i} T^{i}\left({ }^{\circ} \mathrm{C}\right) \\
c_{a}^{0}=1.31678 \times 10^{-1} & c_{b}^{0}=-1.86731 \times 10^{-2} & c_{c}^{0}=2.88841 \times 10^{-3} \\
c_{a}^{1}=-8.36829 \times 10^{-4} & c_{b}^{1}=3.9022 \times 10^{-4} & c_{c}^{1}=-6.70405 \times 10^{-5} \\
c_{a}^{2}=3.07179 \times 10^{-6} & c_{b}^{2}=-2.62611 \times 10^{-6} & c_{c}^{2}=5.65666 \times 10^{-7} \\
c_{a}^{3}=1.46701 \times 10^{-9} & c_{b}^{3}=4.40918 \times 10^{-9} & c_{c}^{3}=-1.34012 \times 10^{-9}
\end{array}
$$

Coefficients a, b, and c vary with temperature, as shown in Fig. 9-2.

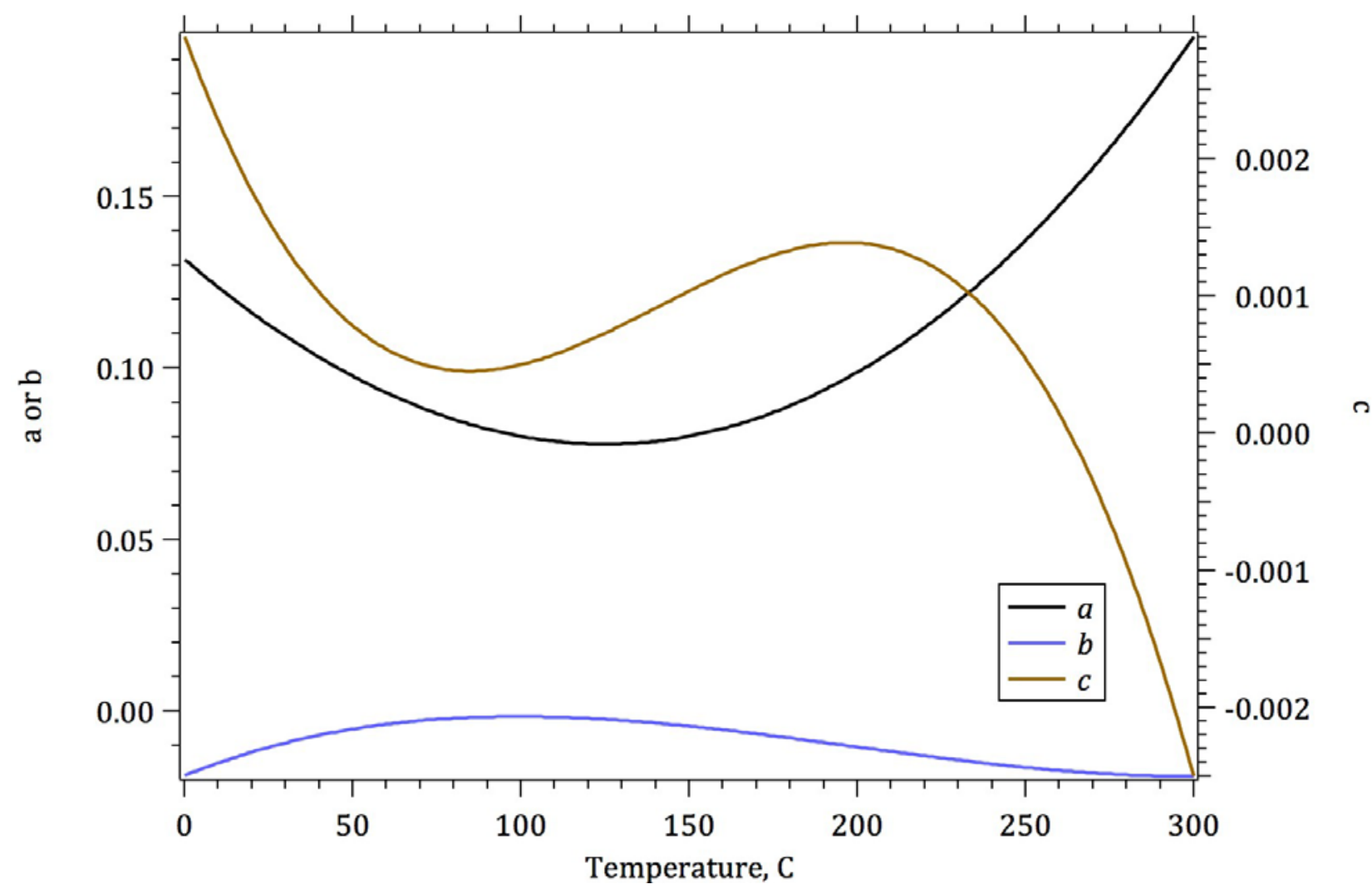

Figure 9-2. Temperature dependence on the coefficients in the B-dot equation 


\subsection{Solution Metrics for Benchmark Problem 5}

Two simulations were to be conducted for a period of 10 years: 1) injection of "pure" water and 2) "recycled" water. The following results are to be reported for both simulations:

1. Temperature ${ }^{\circ} \mathrm{C}$, porosity, fracture permeability $\left(\mathrm{m}^{2}\right)$, and pressure $(\mathrm{MPa})$ versus distance at 10 years

2. Aqueous concentrations (mole/liter) of $\mathrm{H}+, \mathrm{OH}-, \mathrm{HSiO} 3-$, and $\mathrm{SiO} 2(\mathrm{aq})$ as a function of time at $3.0 \mathrm{~m}$ from the inlet

3. Change in amorphous silica abundance as a function of time at $3.0 \mathrm{~m}$ from the inlet

\subsection{Results for Benchmark Problem 5}

The solutions for temperature, pressure, permeability, and porosity across the length of the fracture for the "pure" water scenario at 10 years is shown for the 3 participating teams in Figs. 9-3 and 9-4, respectively. The temperature profiles show heating of the injected water from $65^{\circ} \mathrm{C}$ to nearly $120^{\circ} \mathrm{C}$, with a nearly linear increase across the fracture length. Pressure profiles, however, show sharper gradients in pressure decline near the inlet side of the fracture, transitioning to linear profiles. Both pressure and temperature trends predicted by the three teams are in good agreement. After ten years of injecting fresh water, the fracture porosity is higher across the entire length, with a peak occurring near $30-\mathrm{m}$ from the inlet. Increases in porosity with the dissolution of amorphous silica yield sharp increases in permeability with logarithmic profiles similar to those for porosity, as expected from the permeability to porosity relationship shown in Eqn. (9.2). The porosity and permeability profiles at 10 years result from dissolution of the amorphous silica at about 15 meters from the inlet. This increase in porosity results in large increases in permeability and a migration of the dissolution front downstream. A comparison of the "pure" water scenarios in terms of results for temperature, pressure, fracture permeability, and fracture porosity are provided in Figs. 9-5 to 9-8, respectively. For all output parameters, the uncertainty in simulation results increase across the length of the domain, which is expected with fixed inlet conditions. Maximum uncertainties in temperature, pressure, permeability, and porosity for the "pure" water scenario at 10 years is $3.65^{\circ} \mathrm{C}, 0.046 \mathrm{MPa}, 1.1 \mathrm{e}-11 \mathrm{~m}^{2}, 0.029$, respectively. 


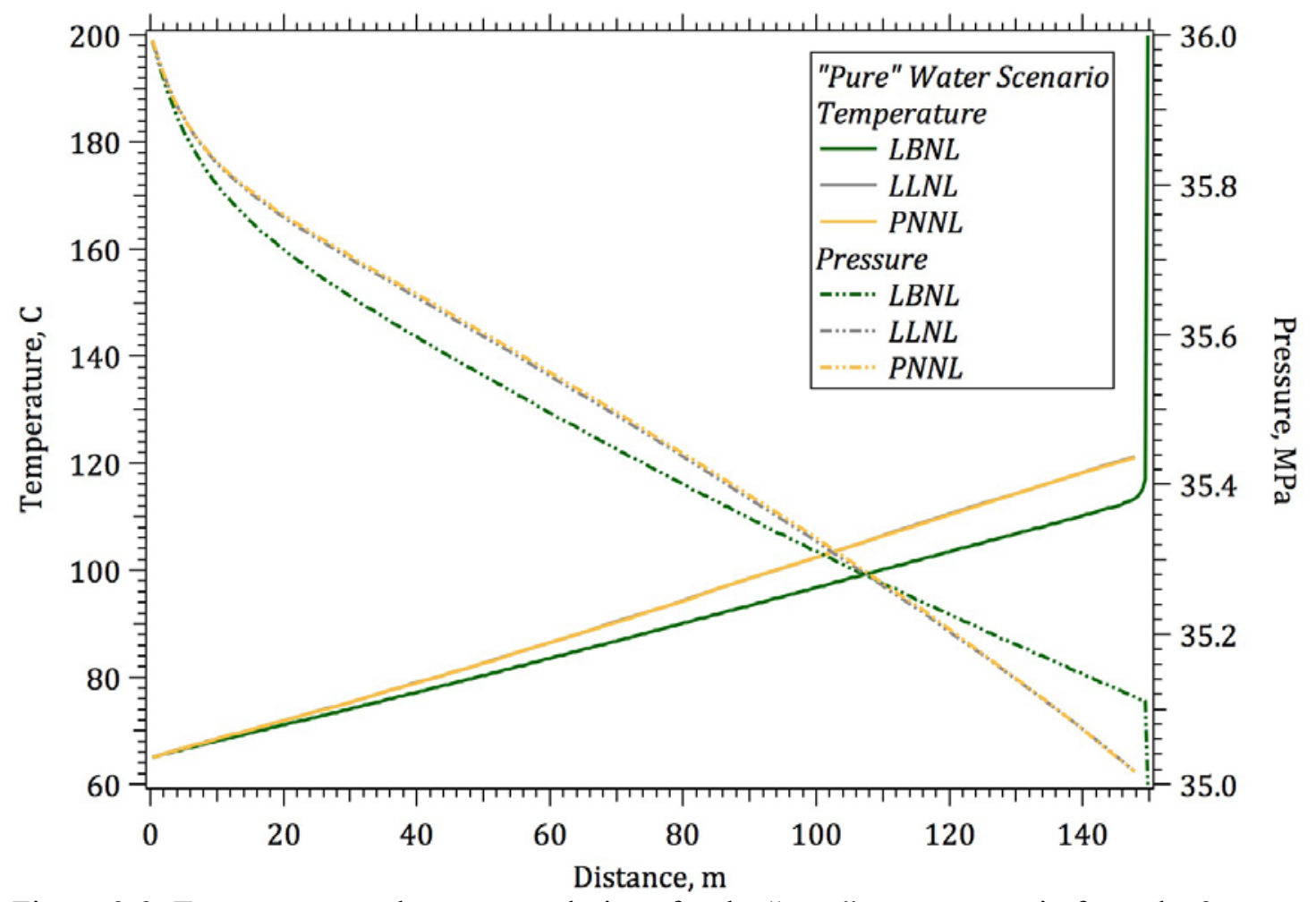

Figure 9-3. Temperature and pressure solutions for the "pure" water scenario from the 3 teams

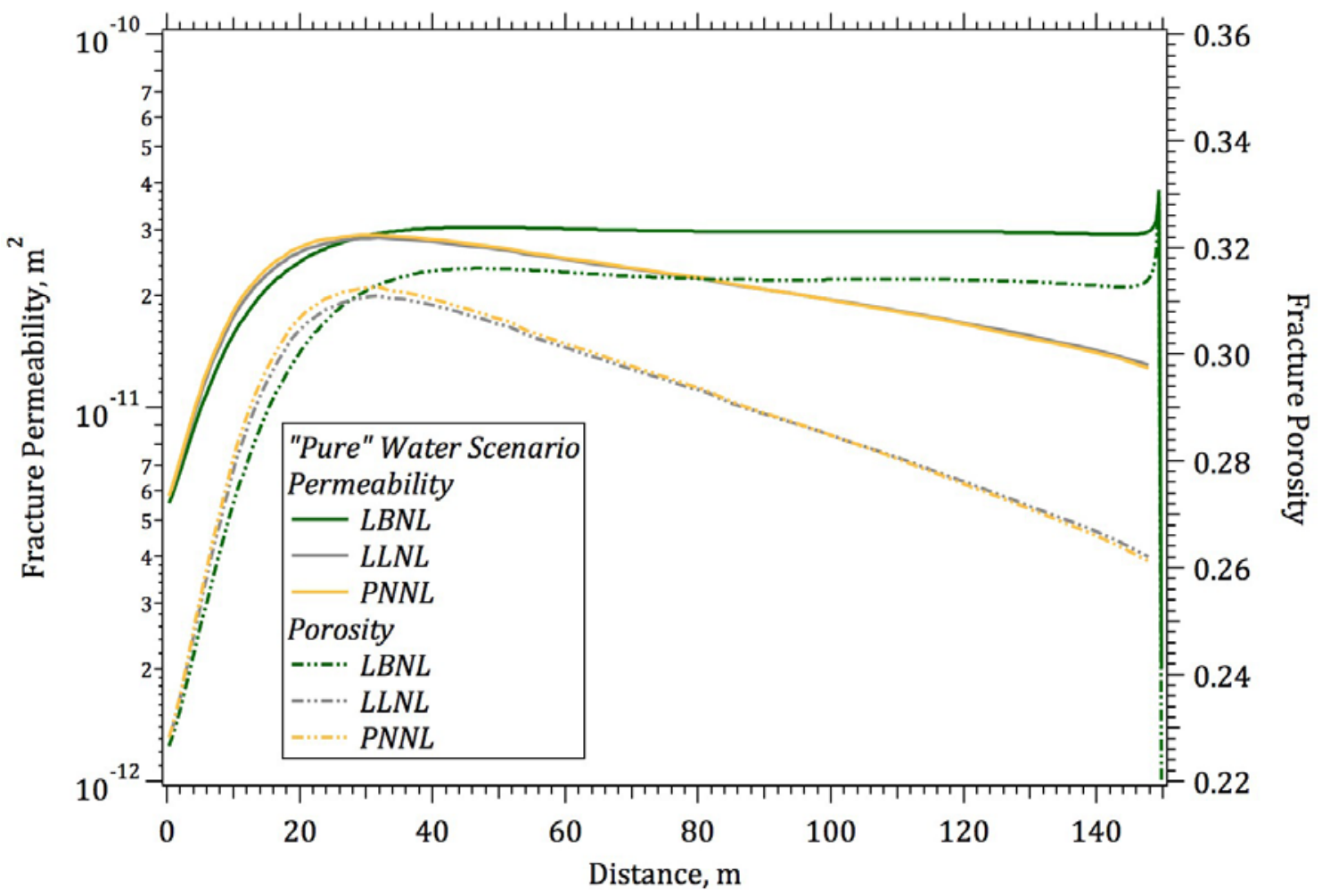

Figure 9-4. Permeability and porosity solutions for the "pure" water scenario from the 3 teams 


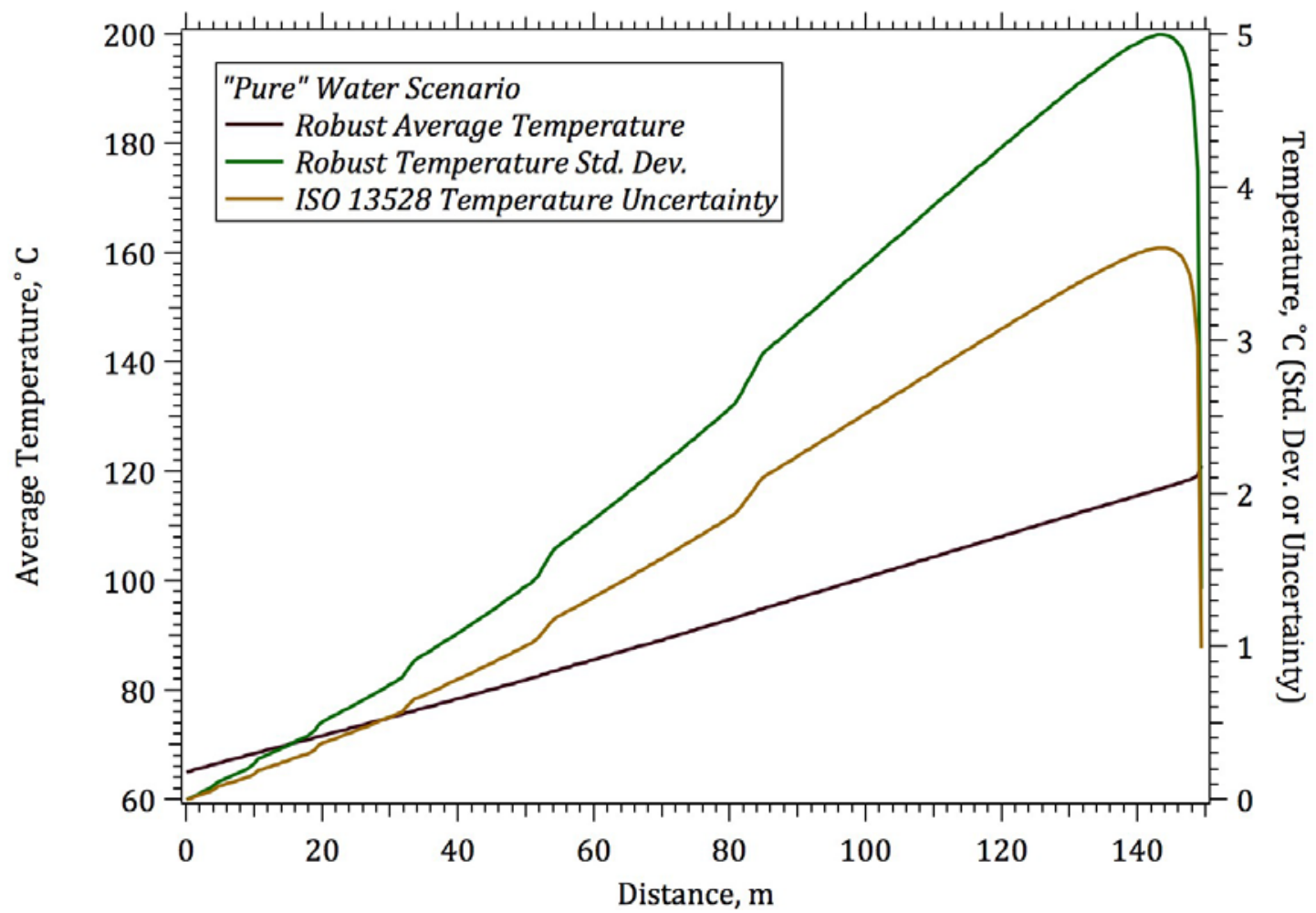

Figure 9-5. Robust average, standard deviation, and ISO 13528 uncertainty in temperature for the "pure" water scenario

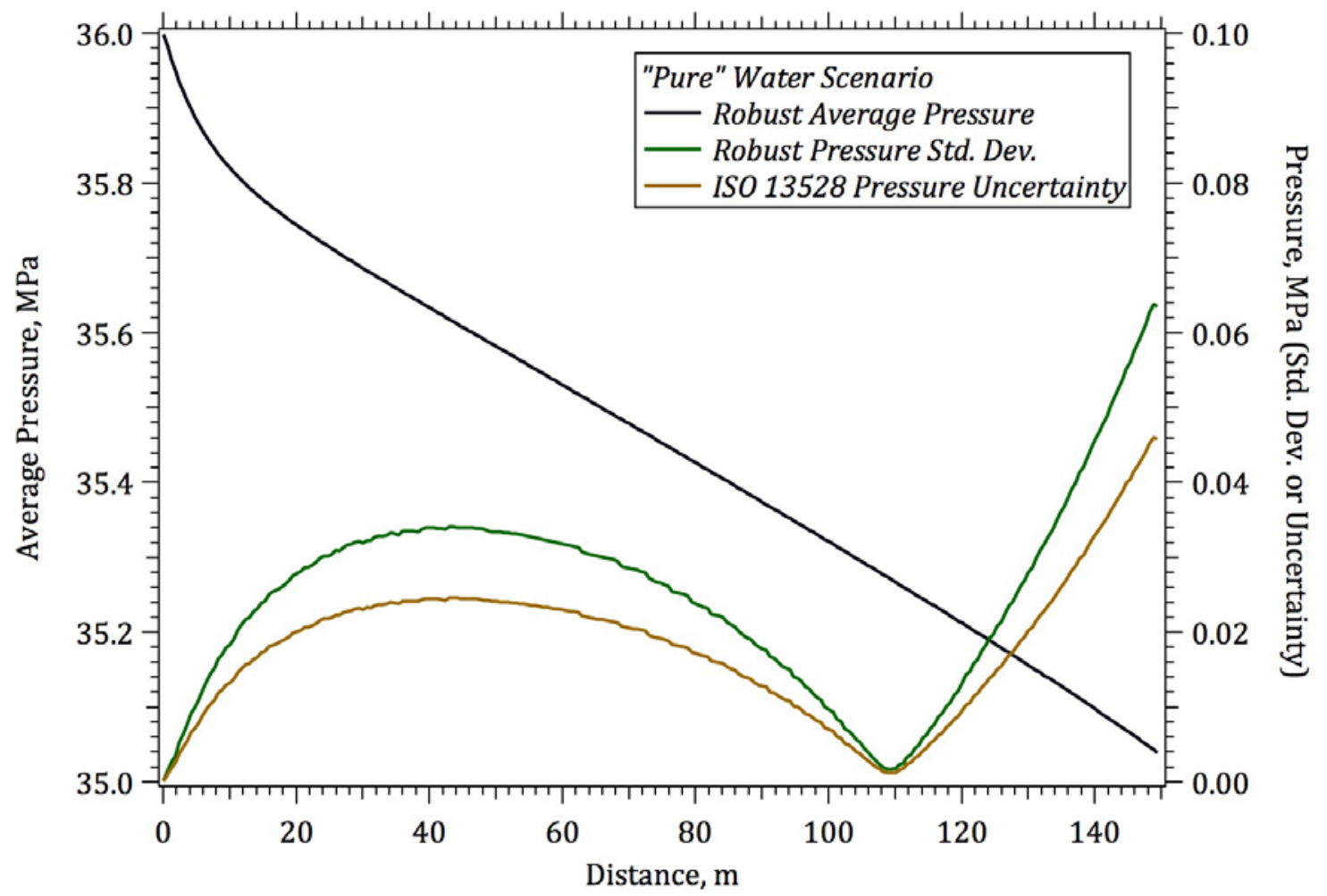

Figure 9-6. Robust average, standard deviation, and ISO 13528 uncertainty in pressure for the "pure" water scenario 


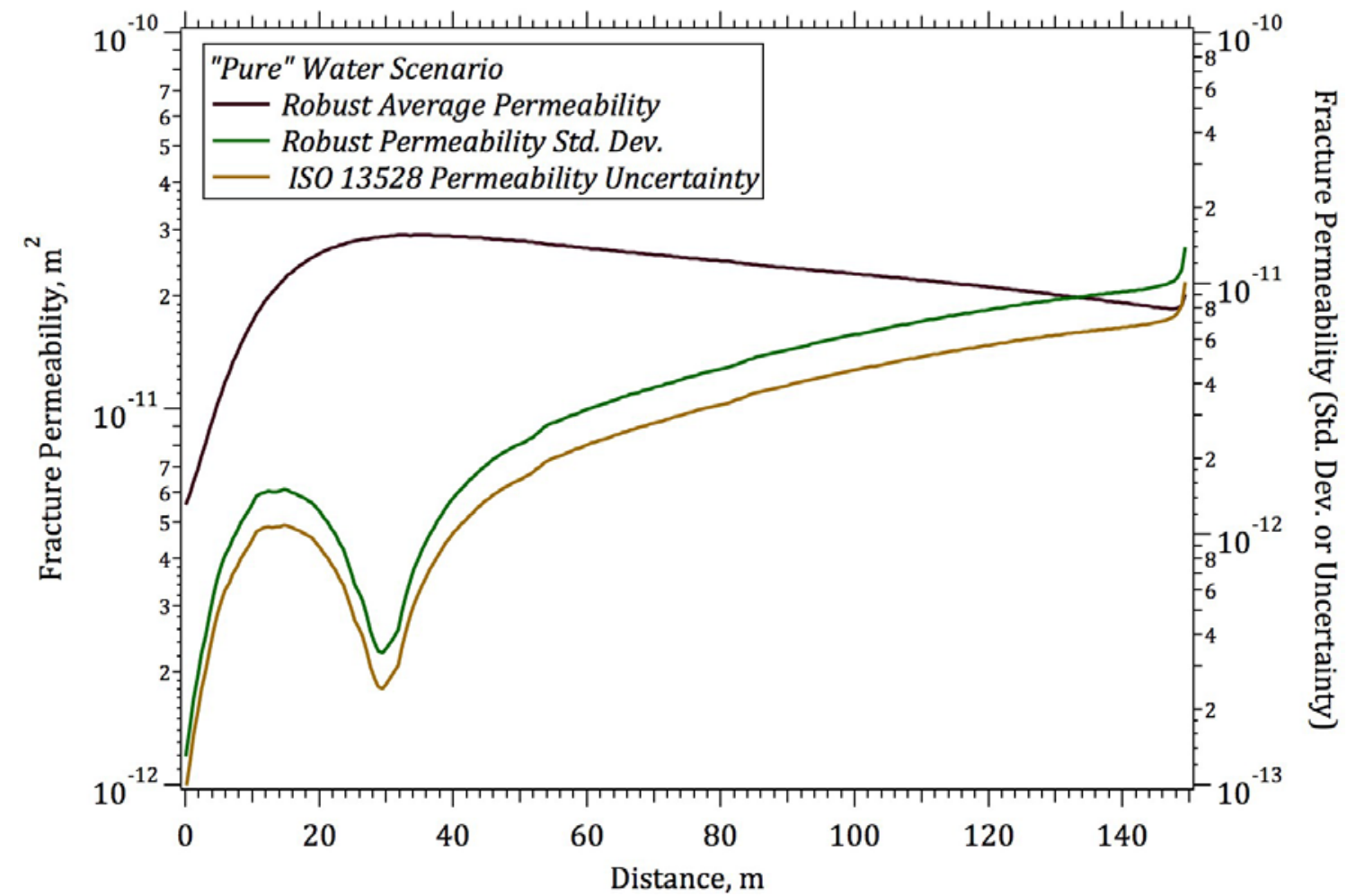

Figure 9-7. Robust average, standard deviation, and ISO 13528 uncertainty in fracture permeability for the "pure" water scenario

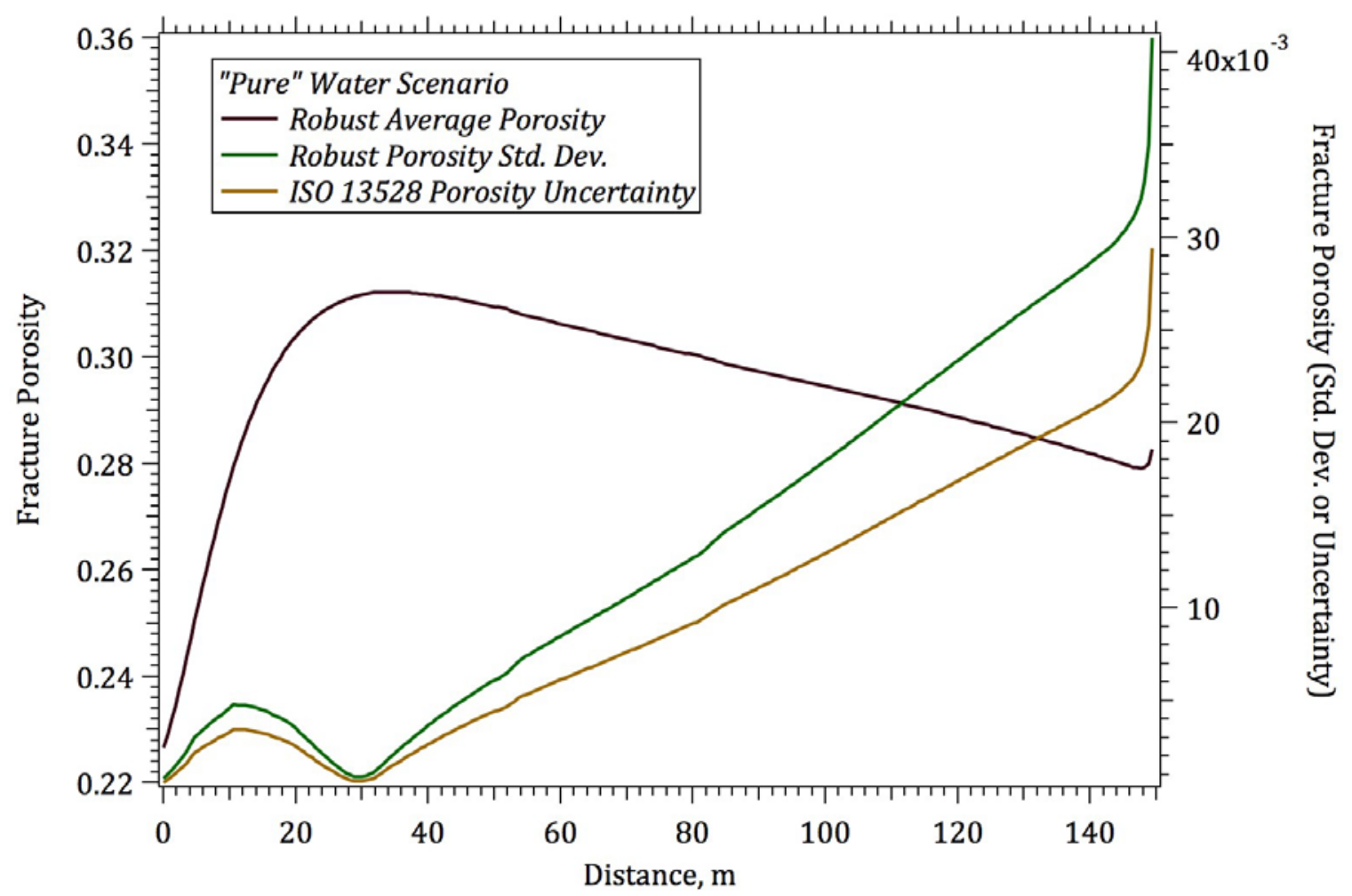

Figure 9-8. Robust average, standard deviation, and ISO 13528 uncertainty in fracture porosity for the "pure" water scenario 
The solutions for temperature and pressure and permeability and porosity across the length of the fracture for the "recycle" water scenario at 10 years is shown for the 3 participating teams in Figs. 9-9 and 9-10, respectively. The results for the "recycle" water scenario differ significantly from those of the "pure" water scenario. The temperature profiles show a sharp increase in temperature at the inlet, then an asymptotic increase to the original reservoir temperature of $200^{\circ} \mathrm{C}$. The pressure profiles at 10 years show sharp drop from the inlet boundary pressure of $36 \mathrm{MPa}$ to $35 \mathrm{MPa}$, the outlet boundary pressure at the inlet, with a flat profile across the length of the fracture. This rather unusual profile is the result of plugging of the fracture with precipitated amorphous silica. For this problem plugging of the fracture occurs at the critical porosity of 0.16, which occurs near the inlet as shown in Fig. 9-10. The permeability and porosity profiles along the length of the fracture are those that develop between the start of the simulation and ultimate plugging of the fracture. Precipitation of the amorphous silica from the supersaturated inlet solution starts to occur near 5 meters from the inlet. This precipitation causes a reduction in the flow rate, leading to migration of the fracture plugging region toward the inlet. Once the fracture becomes completely plugged (i.e., permeability $\sim 0$ ) after a few years, the profiles at 10 years reflect this absence of flow along the fracture. This simulation is similar to the fracture plugging experiment and modeling discussed in (Dobson et al. 2003). A comparison of the "recycle" water scenarios in terms of results for temperature, fracture permeability, and fracture porosity are provided in Figs. 9-11 to 9-13, respectively. Uncertainty in the simulation results are highest for temperature, permeability and porosity where precipitation of the amorphous silica was the most rapid earlier in the simulation. Maximum uncertainties in temperature, permeability, and porosity for the "recycle" water scenario at 10 years is $5.1^{\circ} \mathrm{C}, 9.9 \mathrm{e}-13 \mathrm{~m}^{2}, 0.0091$, respectively.

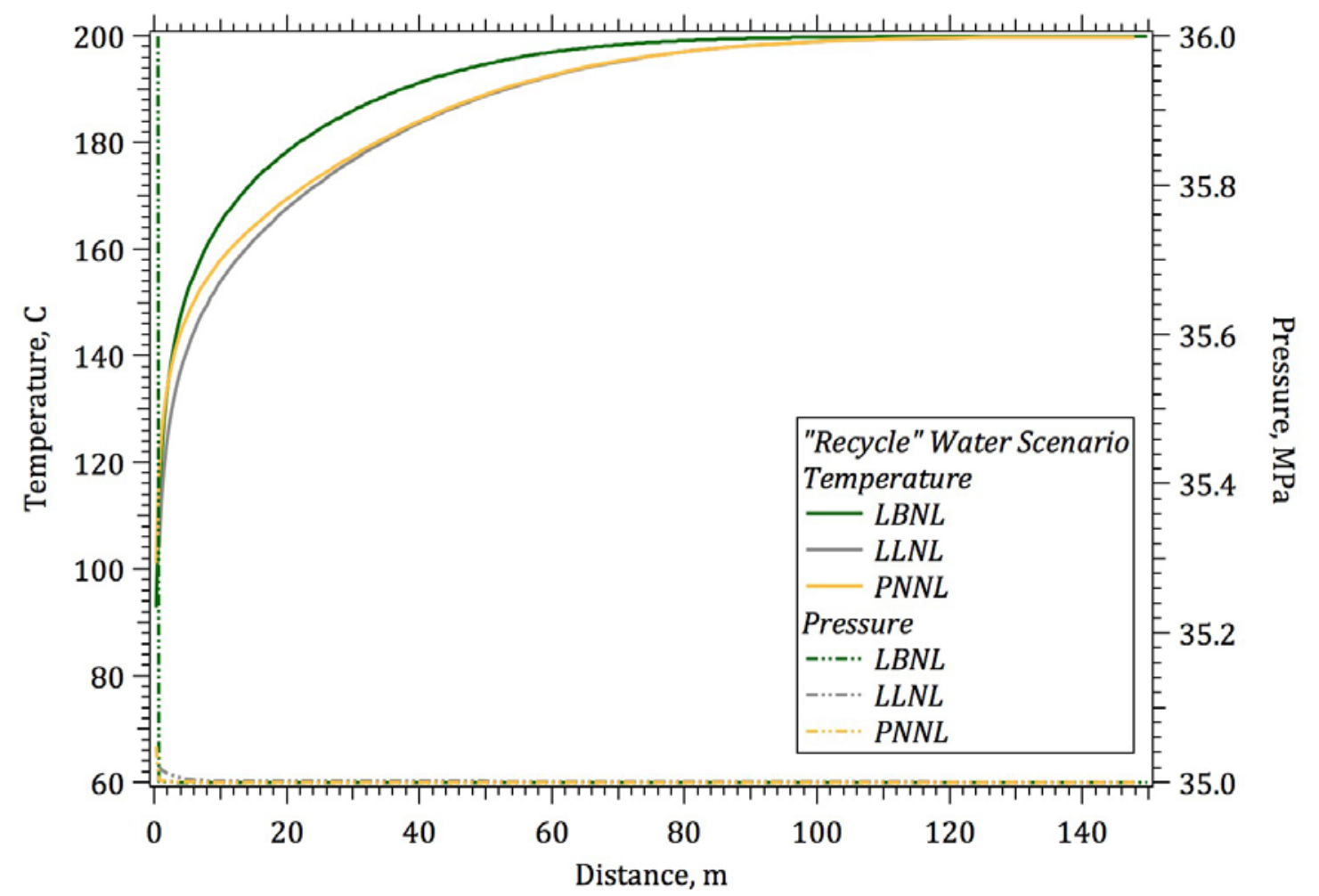

Figure 9-9. Temperature and pressure solutions for the "recycle" water scenario from the 3 teams 


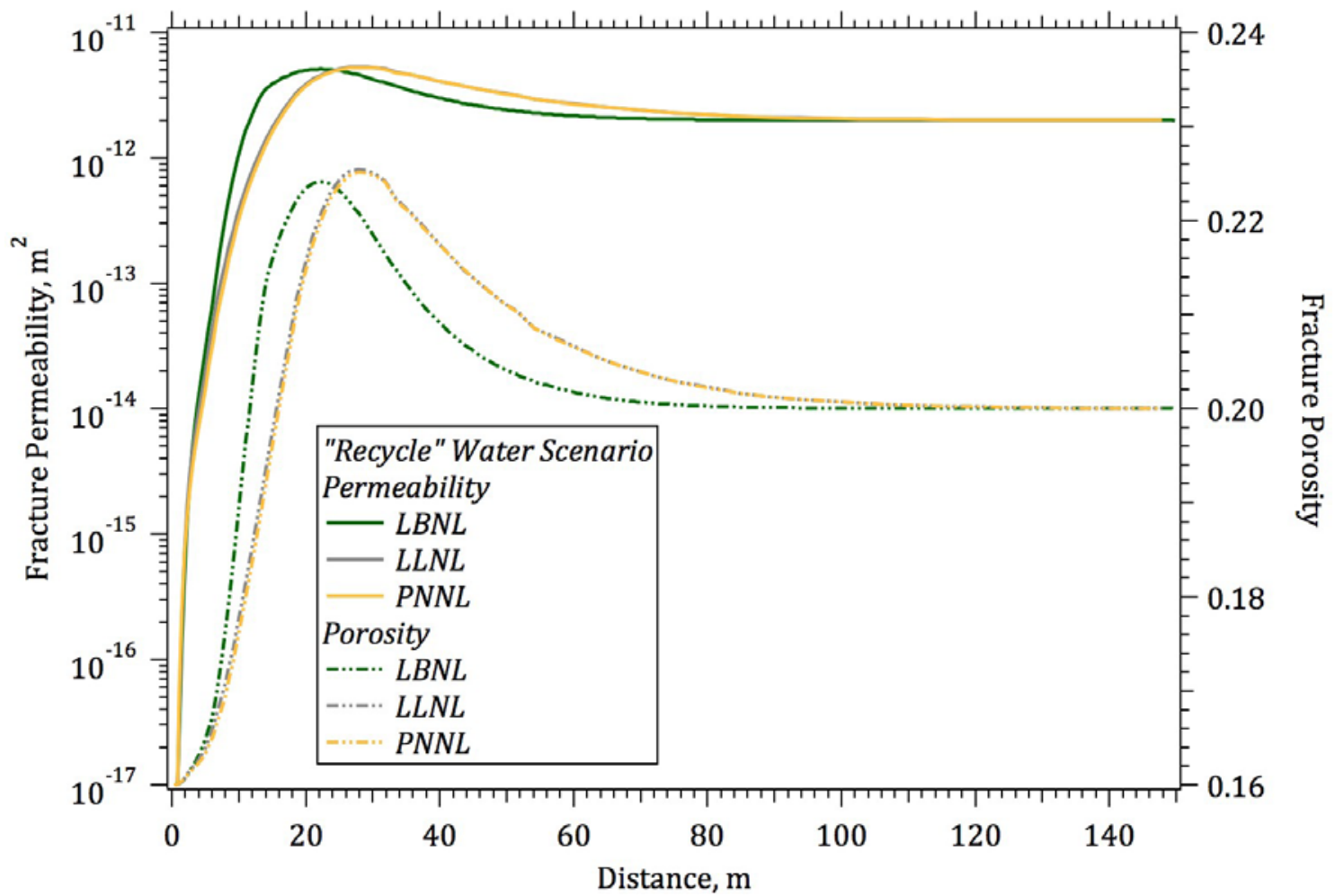

Figure 9-10. Permeability and porosity solutions for the "recycle" water scenario from the 3 teams

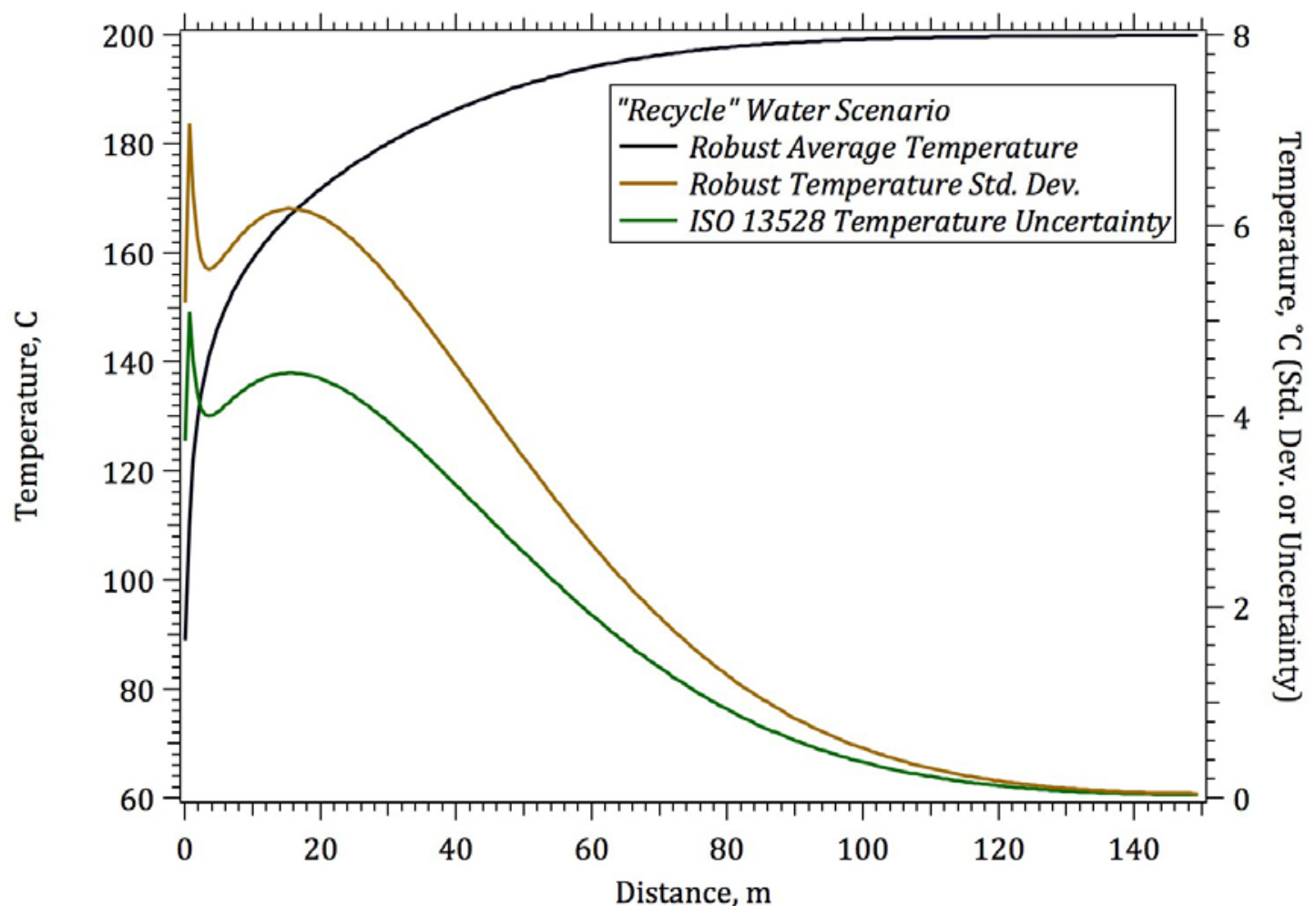

Figure 9-11. Robust average, standard deviation, and ISO 13528 uncertainty in temperature for the "recycle" water scenario 


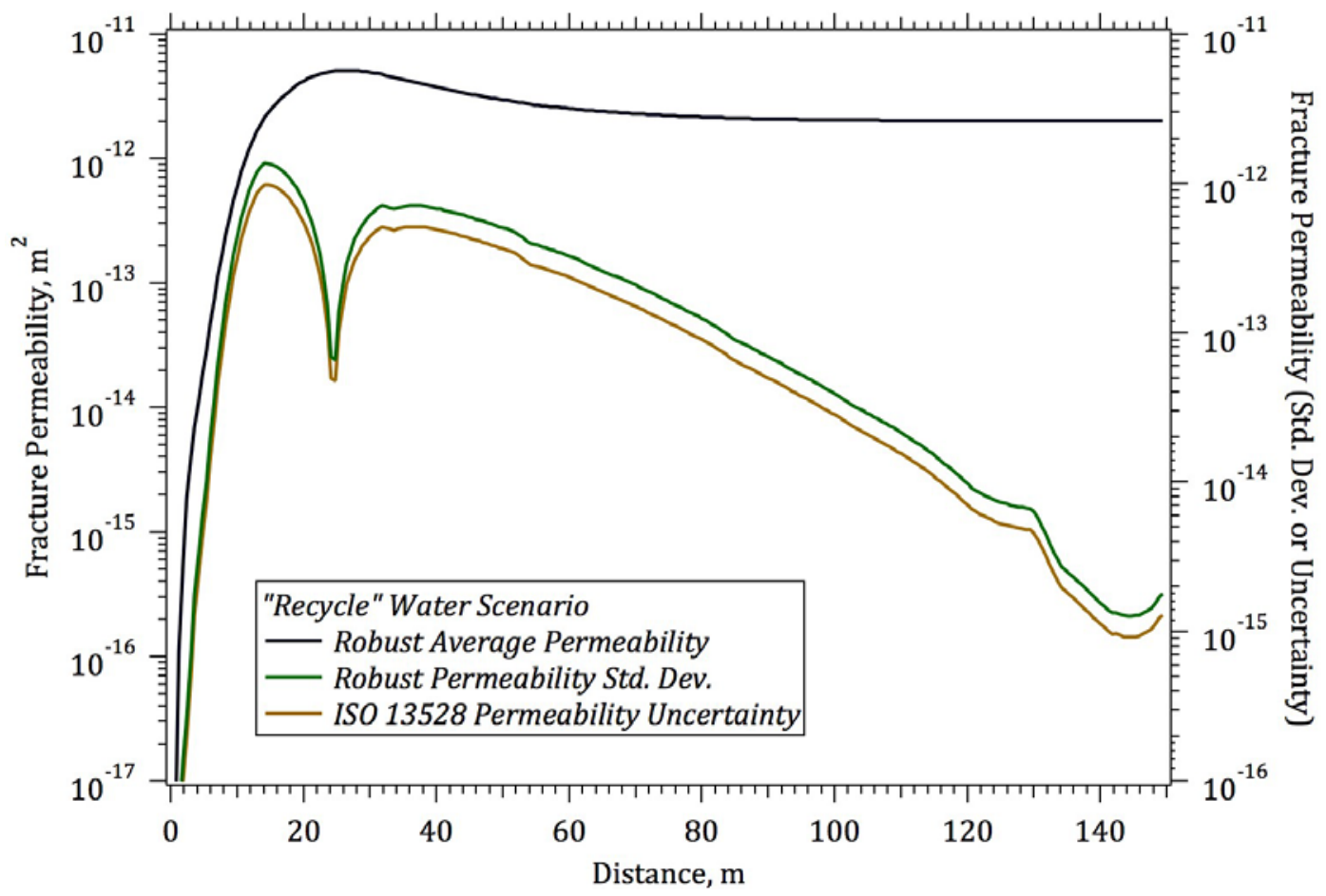

Figure 9-12. Robust average, standard deviation, and ISO 13528 uncertainty in permeability for the "recycle" water scenario

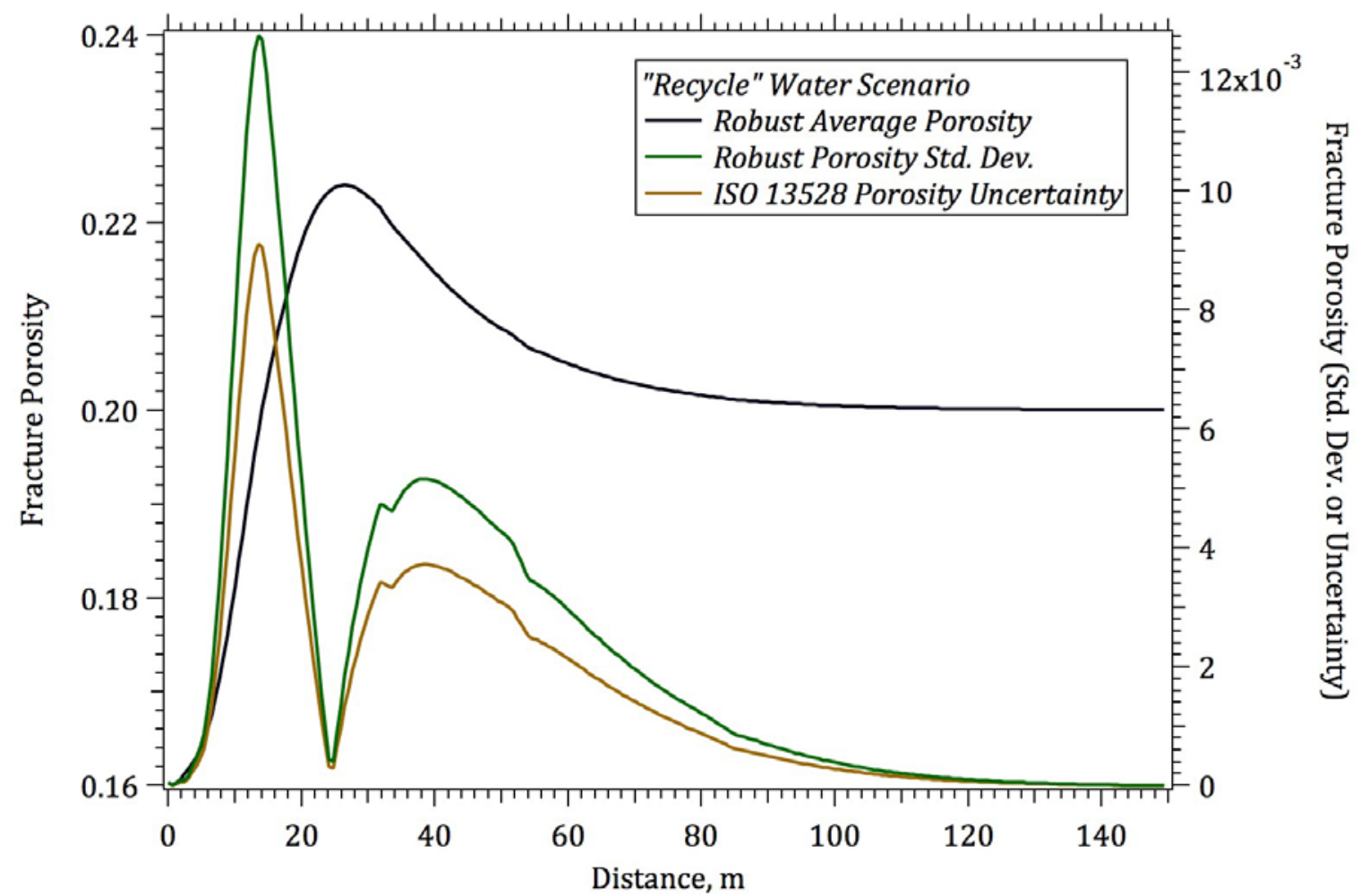

Figure 9-13. Robust average, standard deviation, and ISO 13528 uncertainty in porosity for the "recycle" water scenario 
The solutions for aqueous concentrations of $\mathrm{H}+, \mathrm{HSiO} 3-$, and $\mathrm{SiO} 2(\mathrm{aq})$ as a function of time at $3.0 \mathrm{~m}$ from the inlet for the "pure" water scenario are shown in Fig. 9-14 for the 3 participating teams.

Dissolved concentrations of $\mathrm{SiO} 2(\mathrm{aq})$ increase rapidly from the start of the simulation with the dissolution of amorphous silica between the inlet and the $3.0-\mathrm{m}$ point. Once saturation conditions are reached the concentration plateaus, but continued dissolution results in increasingly higher flow rates and lower concentrations. Similar trends are seen in the $\mathrm{H}+$ and $\mathrm{HSiO} 3$ - concentrations. The change in amorphous silica abundance, expressed as the $\mathrm{SiO} 2(\mathrm{am})$ bulk concentration in $\mathrm{mol} / \mathrm{L}$ (bulk), as a function of time at $3.0 \mathrm{~m}$ from the inlet for the "pure" water scenario is shown in Fig. 9-15. The slope of the plot shown in Fig. 9-15 provides an indication of the rate of dissolution of the amorphous silica, and agrees with the dissolved concentrations of species shown in Fig. 9-14, rapid rates of dissolution early in time, followed by more moderate rates of dissolution as the flow rate through the fracture increases, due to the constant pressure boundary conditions and evolving fracture porosity and permeability. A comparison of the "pure" water scenarios in terms of results for bulk concentrations of $\mathrm{SiO} 2(\mathrm{am})$ and aqueous concentrations of $\mathrm{SiO} 2(\mathrm{aq})$ are provided in Fig. 9-16. Uncertainty in the simulation results at $3.0 \mathrm{~m}$ from the inlet are relatively higher than those shown for the results after 10 years. Accurately capturing the strong dissolution of the amorphous silica between the inlet and the $3.0-\mathrm{m}$ mark, requires high grid resolution. The level of uncertainty is reflective of the different gridding schemes used. Maximum uncertainties in $\mathrm{SiO} 2(\mathrm{aq})$ and change in $\mathrm{SiO} 2(\mathrm{am})$ for the "pure" water scenario are $7.52 \mathrm{e}-4 \mathrm{~mol} / \mathrm{L}(\mathrm{aq})$ and $0.290 \mathrm{~mol} / \mathrm{L}$ bulk, respectively.

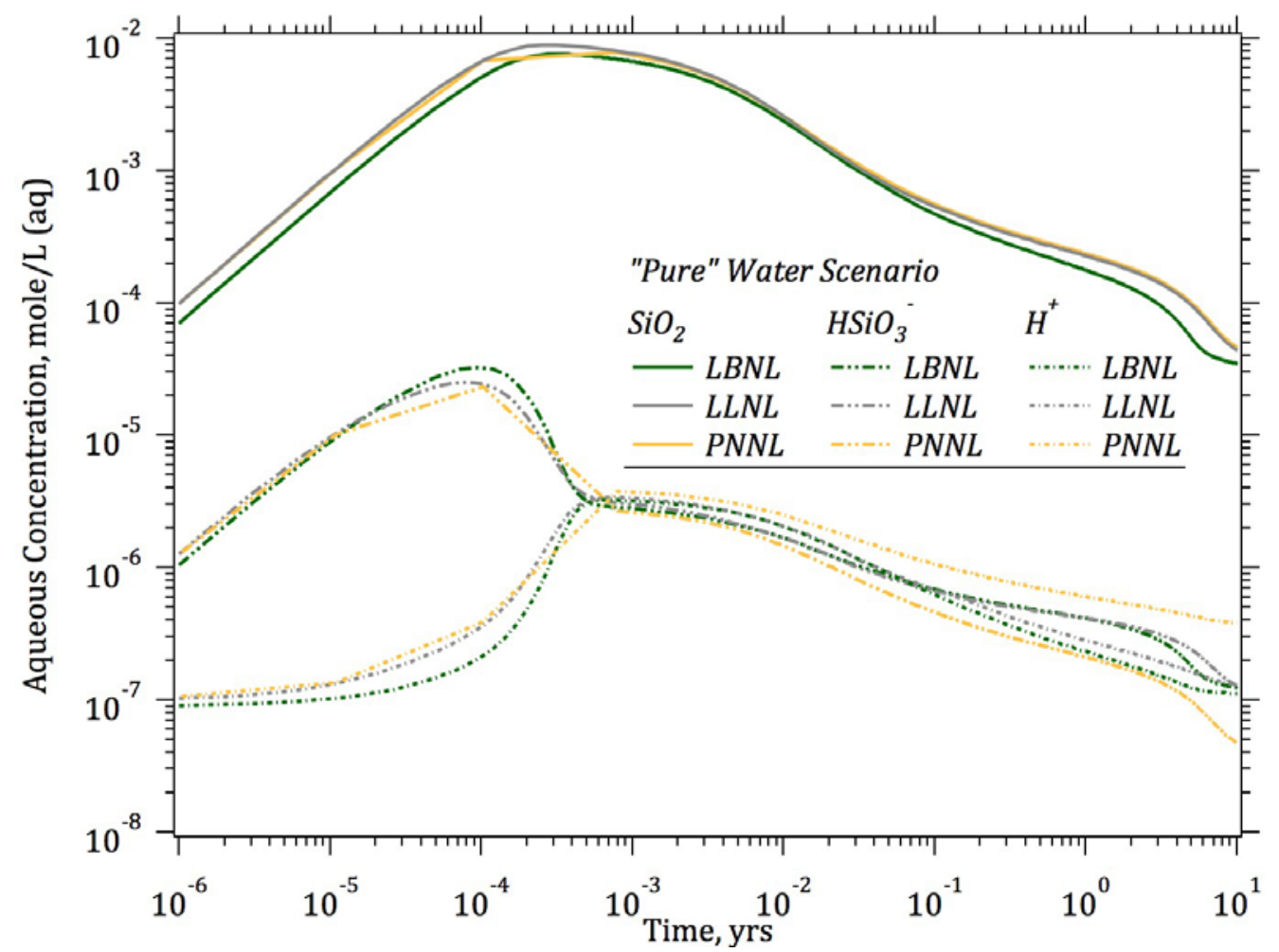

Figure 9-14. Aqueous concentrations (mol/L (aqu)) of $\mathrm{H}+, \mathrm{HSiO}-$, and $\mathrm{SiO} 2(\mathrm{aq})$ as a function of time at $3.0 \mathrm{~m}$ from the inlet for the "pure" water scenario 


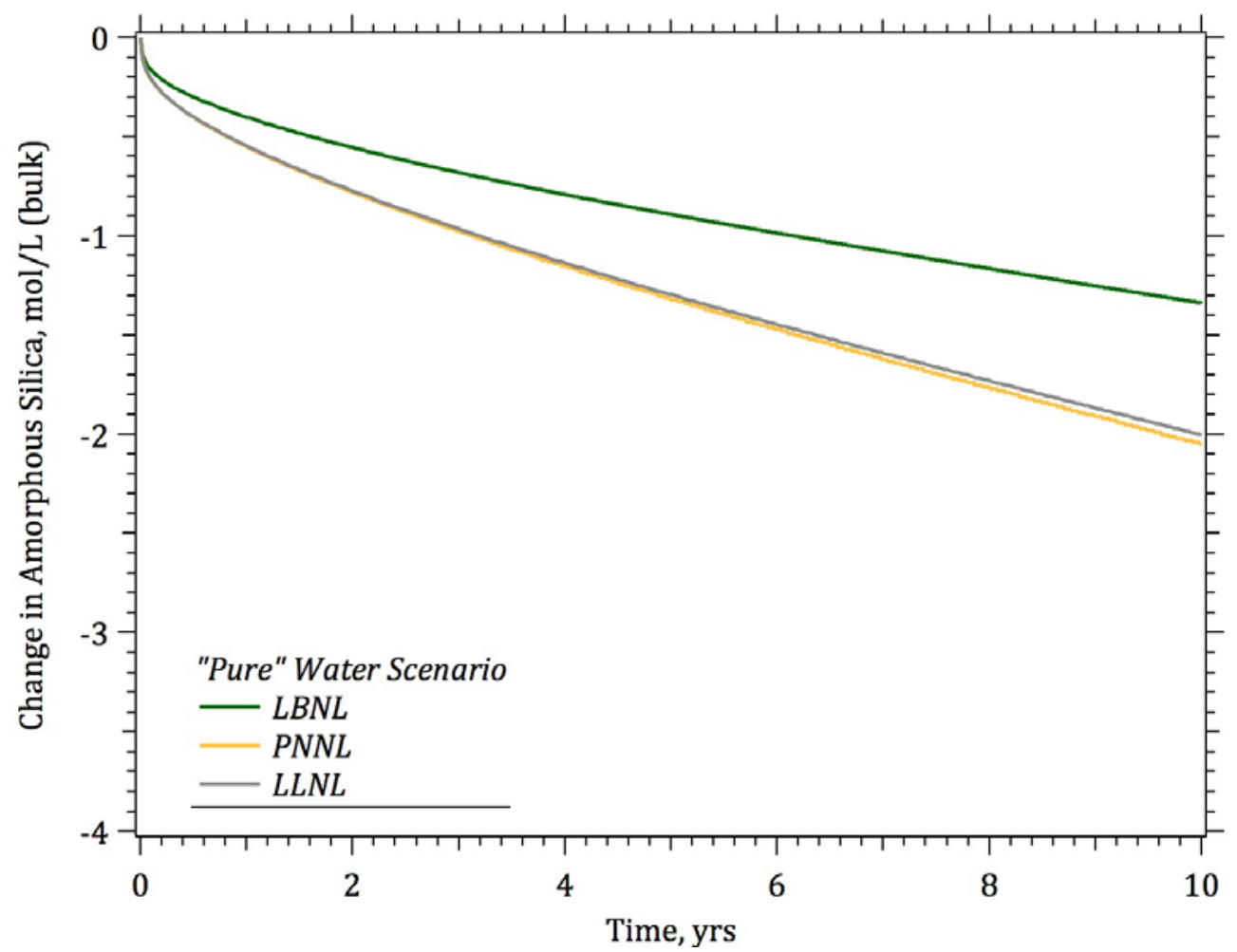

Figure 9-15. Bulk concentrations (mol/L (bulk)) of $\mathrm{SiO} 2(\mathrm{am})$ as a function of time at $3.0 \mathrm{~m}$ from the inlet for the "pure" water scenario

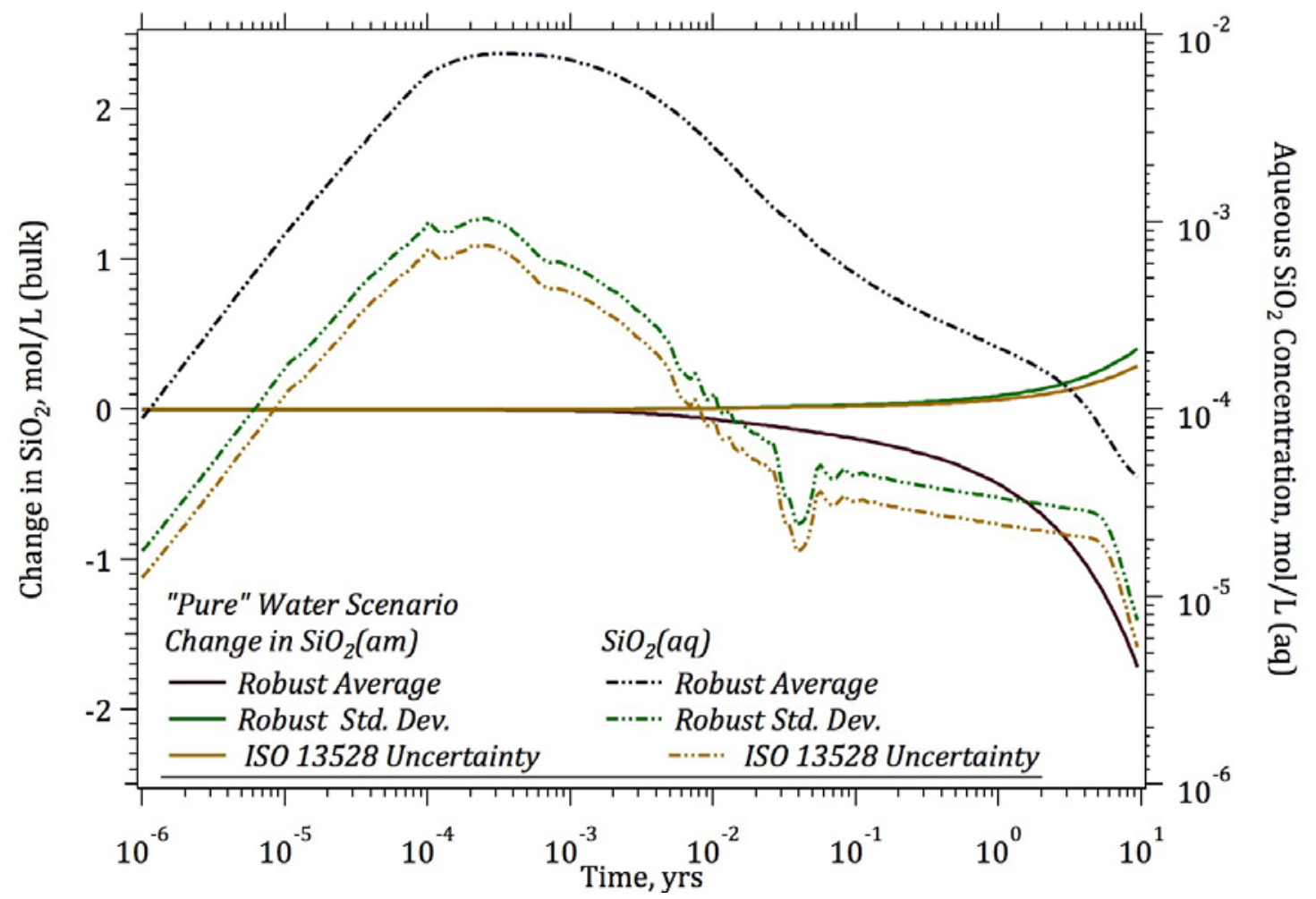

Figure 9-16. Robust average, standard deviation, and ISO 13528 uncertainty in bulk conc. of $\mathrm{SiO} 2(\mathrm{am})$ and aqueous conc. of $\mathrm{SiO} 2(\mathrm{aq})$ for the "pure" water scenario. 
The solutions for aqueous concentrations of $\mathrm{H}+, \mathrm{HSiO} 3-$, and $\mathrm{SiO} 2(\mathrm{aq})$ as a function of time at $3.0 \mathrm{~m}$ from the inlet for the "recycle" water scenario are shown in Fig. 9-17 for the 3 participating teams. Early response of the concentrations of dissolved $\mathrm{SiO} 2(\mathrm{aq}), \mathrm{H}+$, and $\mathrm{HSiO} 3-$ are similar to those for the "pure" water scenario, but the concentrations are being achieved via the precipitation of $\mathrm{SiO} 2(\mathrm{am})$ from the supersaturated inlet fluid. Flow blockage can be observed in the concentration plots, occurring after 1.5 years in the LBNL simulation and 4.0 years in the PNNL simulation. Differences in these blockage times are likely due to differences in grid spacing between the two simulations, as discussed below.

The change in amorphous silica abundance, expressed as the $\mathrm{SiO} 2(\mathrm{am})$ bulk concentration in $\mathrm{mol} / \mathrm{L}$ (bulk), as a function of time at $3.0 \mathrm{~m}$ from the inlet for the "recycle" water scenario is shown in Fig. 9-18. Precipitation of amorphous silica occurs at a nearly constant rate from the start of the simulation as indicated by the constant slopes, shown in Fig. 9-18. The rate of precipitation predicted by the LBNL simulation is slightly faster than that for the PNNL simulation, leading to an earlier flow blockage time, as indicated by the transition from a constant rate precipitation to a halt in precipitation. The constant rate of precipitation is an anticipated response of the system, considering that the inlet fluid has constant concentrations of dissolved species in a supersaturated state. A comparison of the "recycle" water scenarios in terms of results for bulk concentrations of $\mathrm{SiO} 2(\mathrm{am})$ and aqueous concentrations of $\mathrm{SiO} 2(\mathrm{aq})$ are provided in Fig. 9-19. Maximum uncertainties in $\mathrm{SiO} 2(\mathrm{aq})$ and change in $\mathrm{SiO}$ (am) for the "recycle" water scenario are $2.34 \mathrm{e}-3 \mathrm{~mol} / \mathrm{L}(\mathrm{aq})$ and $0.247 \mathrm{~mol} / \mathrm{L}$ bulk, respectively.

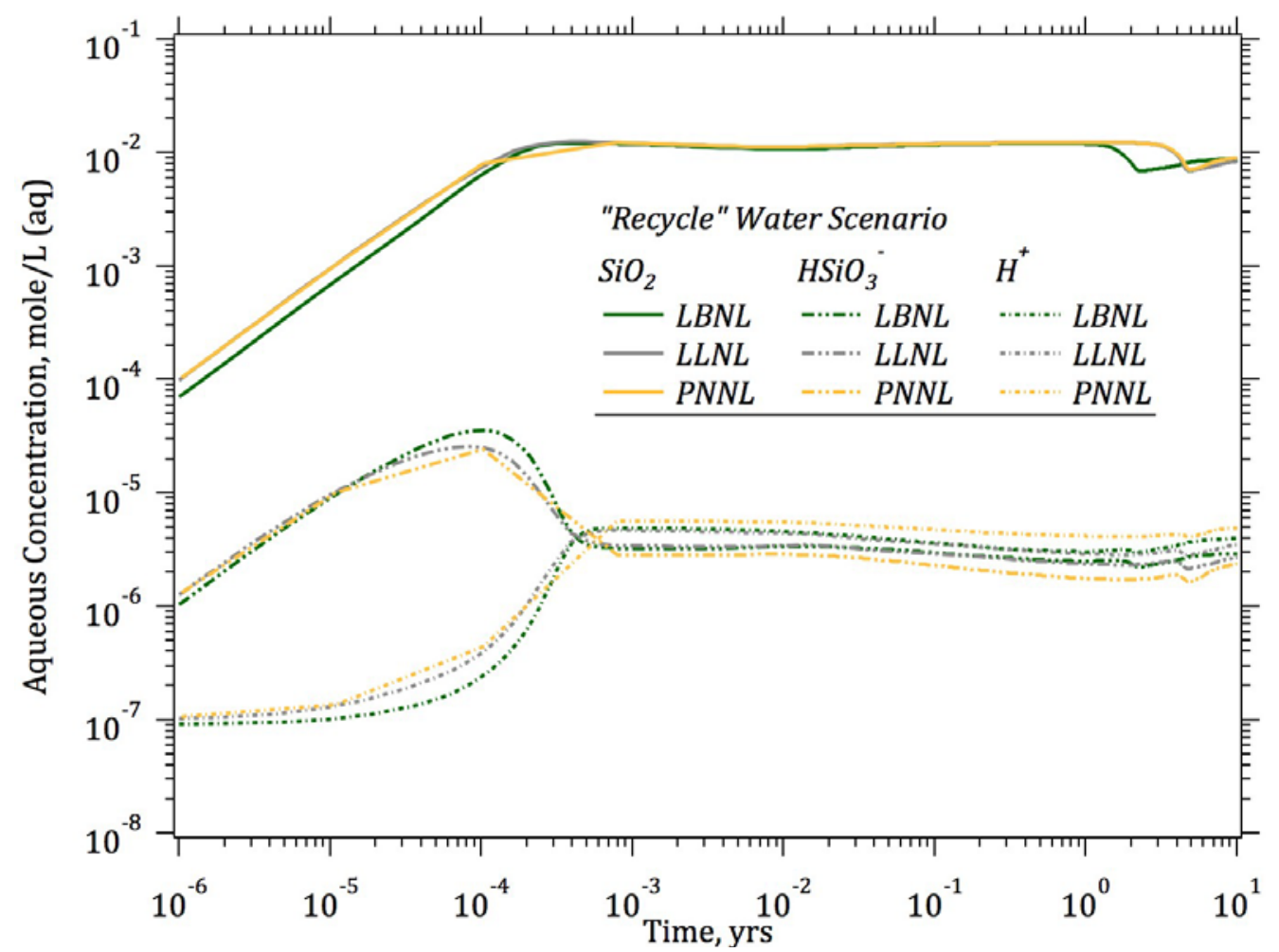

Figure 9-17. Aqueous concentrations (mol/L (aqu)) of $\mathrm{H}+, \mathrm{HSiO} 3-$, and $\mathrm{SiO} 2(\mathrm{aq})$ as a function of time at $3.0 \mathrm{~m}$ from the inlet for the "recycle" water scenario 


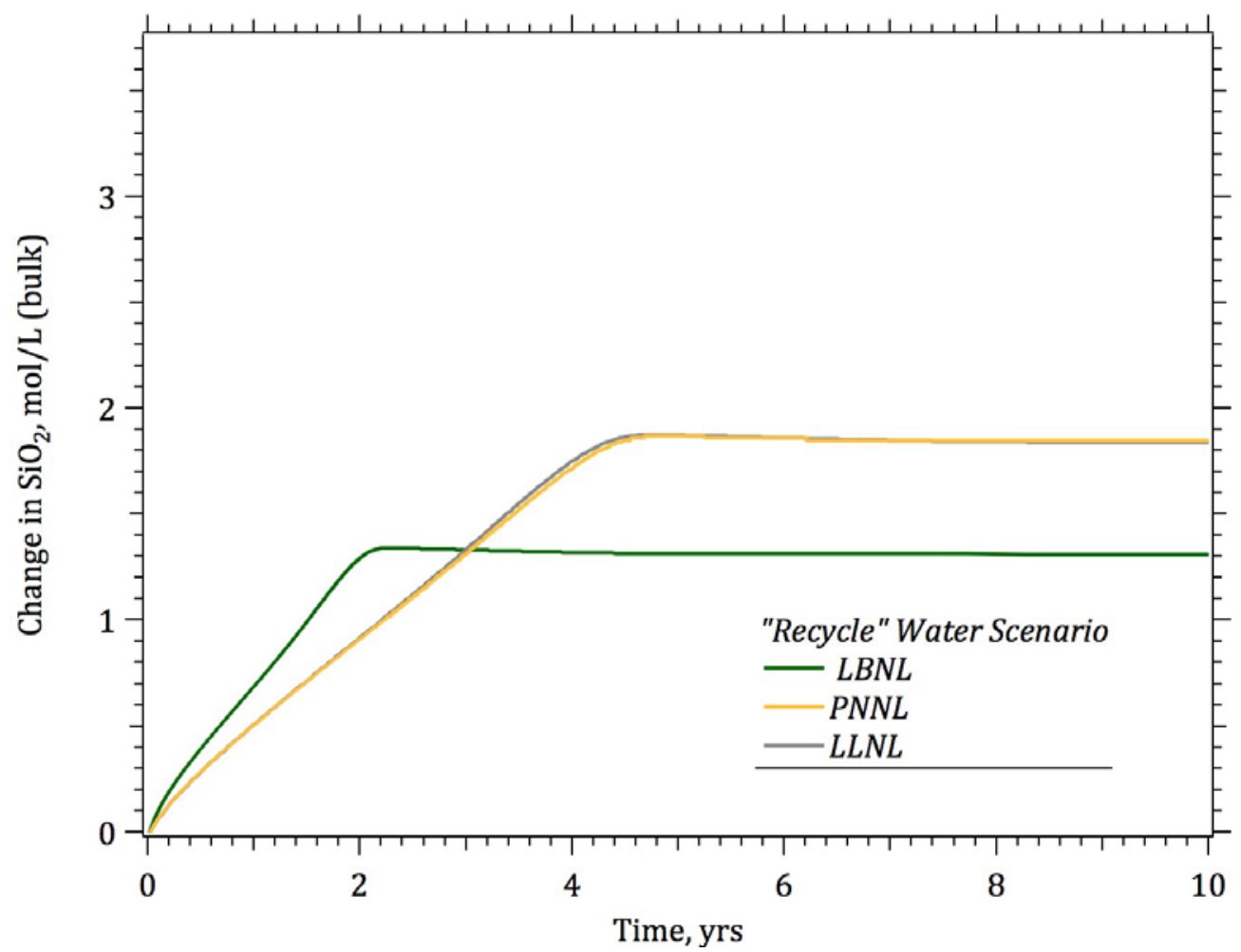

Figure 9-18. Bulk concentrations (mol/L (bulk)) of $\mathrm{SiO} 2(\mathrm{am})$ as a function of time at $3.0 \mathrm{~m}$ from the inlet for the "recycle" water scenario

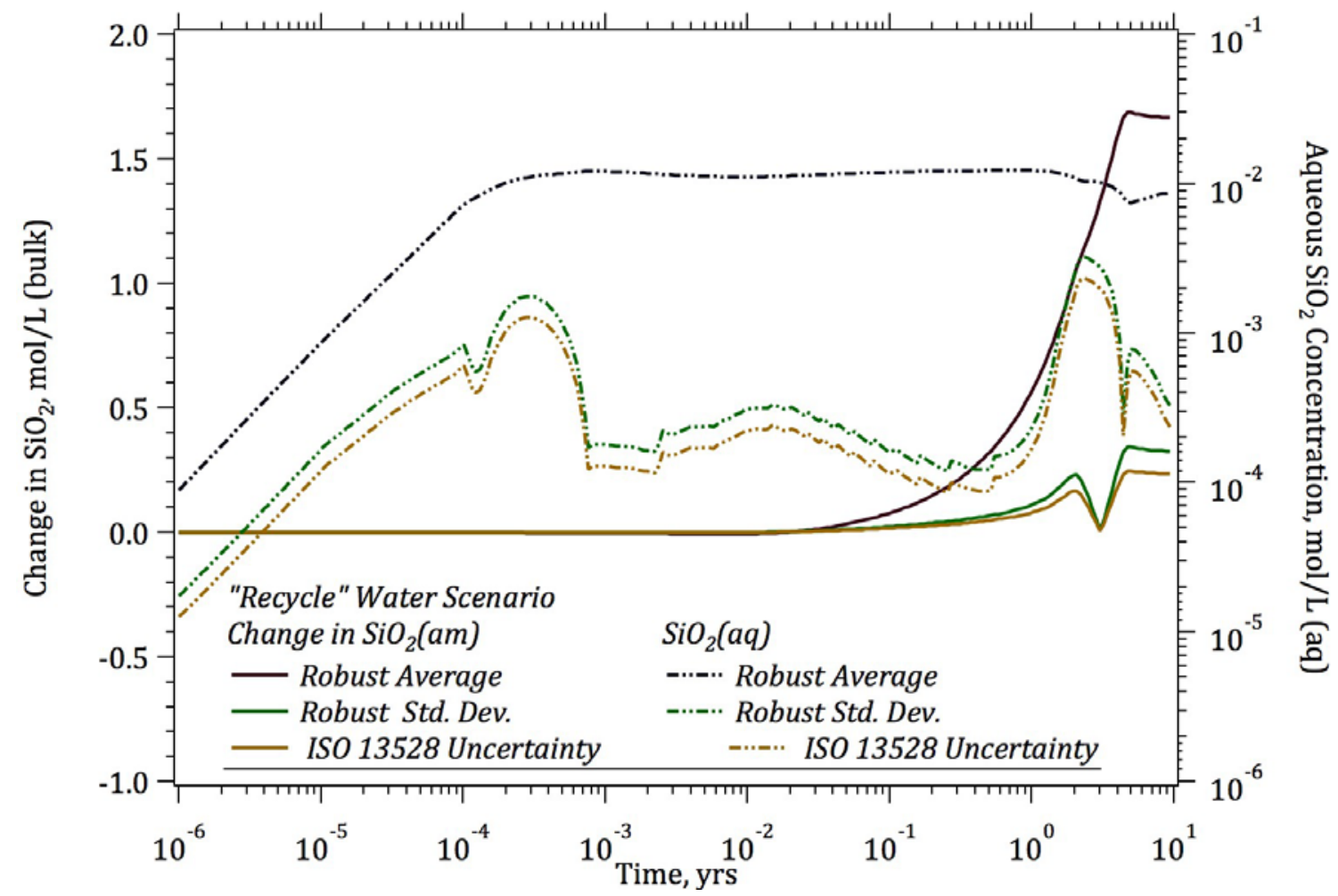

Figure 9-19. Robust average, standard deviation, and ISO 13528 uncertainty in bulk conc. of SiO2(am) and aqueous conc. of $\mathrm{SiO} 2(\mathrm{aq})$ for the "recycle" water scenario 


\subsection{Discussion of Benchmark Problem 5 Results}

The design objectives for Benchmark Problem 5 were to create a single-mineral system with temperature dependent geochemical reaction parameters that yielded sufficiently significant dissolution and precipitation to modify the porosity and permeability of the EGS reservoir. A single-mineral system was selected to allow those simulators without full reactive transport capabilities to participate in the problem, by implementing a more simple geochemistry algorithm/solution scheme into the simulator. Whereas this was attempted by some of the participants, only those participants with existing geochemical simulation capabilities submitted solutions, while some of those with this capability chose not to submit. The unfortunate aspect to the resulting problem is that the solution is sensitive to grid resolution, and that the requirement for a Courant number limit of 1.0 or less for transporting reactive species, compounded with highly resolved grids, translates to long execution times for problem that was designed to be simpler than standard geochemical problems with a suite of reactive minerals. Another unfortunate aspect to the problem is the use of a "recycle" water composition, which was supersaturated. The thermodynamics of the geochemical system was such that $\mathrm{SiO} 2(\mathrm{aq})$ had decreasing solubility with decreasing temperature. Therefore, $\mathrm{SiO} 2(\mathrm{aq})$ leaving the geothermal reservoir would normally precipitate with reducing temperature at the ground surface before being re-injected into the reservoir. For this problem it was assumed that precipitation did not occur during this cooling process and the water was re-injected in a supersaturated state.

Temperature dependent equilibrium constants were a component of this problem, as indicated in Eqn. (9.3) and Table 9-4. The temperature dependence on the equilibrium constant for the two equilibrium reactions is shown in Fig. 9-20. Whereas the concentrations of the dissolved components are in equilibrium at the outlet of the reservoir at the outlet temperature (i.e., approximately $122^{\circ} \mathrm{C}$ in the "pure" water simulation), those species are no longer in equilibrium at the inlet temperature of $65^{\circ} \mathrm{C}$ for the "recycle" water scenario. Therefore, the concentration of $\mathrm{H}+$ was held constant and $\mathrm{OH}-$ was reequilibrated at $65^{\circ} \mathrm{C}$ and the concentration of total dissolved $\mathrm{SiO} 2$ (i.e., $\mathrm{SiO} 2(\mathrm{aq})+\mathrm{HSiO} 3-$ ) was maintained. This yielded the following dissolved species concentrations for the "recycle" water: $\mathrm{H}+$ 2.471e-6 mol/L (aq); OH- 7.774e-08 mol/L (aq); SiO2(aq) 1.305e-2 mol/L (aq); and HSiO3- 2.953e-8 $\mathrm{mol} / \mathrm{L}(\mathrm{aq})$.

Since the amount of amorphous silica precipitated is mainly a function of the silica flux, temperature, and flow rate, as long as the total silica flux is correct, then the outcome of having differing initial speciation should be nearly identical. Likewise as the chemical system was only weakly sensitive to $\mathrm{pH}$, using reequilibrated $\mathrm{OH}$ - concentrations, should not greatly impact the amount of amorphous silica precipitated or the total aqueous silica concentrations. Given that the amorphous silica is supersaturated in the injection fluid $\left(\log (\mathrm{Q} / \mathrm{K})=0.498\right.$ at $\left.65^{\circ} \mathrm{C}\right)$, and at the temperature of fracture $\left(\log (\mathrm{Q} / \mathrm{K})=0.003\right.$ at $\left.200^{\circ} \mathrm{C}\right)$, substantial precipitation is likely very close to the inlet. Since the porosity change is a function of the temperature, and changes in permeability affect the flow rate, which feed back on the rate of precipitation, there is the likelihood of very different results for different model discretizations and implementations of the boundary conditions.

Owing to the fixed pressure boundary condition, higher reductions in permeability near the inlet associated with finer discretization will lead to lower silica fluxes and less overall amounts precipitated. Therefore, results for permeability changes and downstream concentrations are uncertain, unless sensitivity studies are performed with ultra-fine resolution. Therefore, the comparison of codes on this problem would have to be using the exact same gridding and boundary conditions. Even though there are only a few reactions and one mineral in this problem, the silica-supersaturated, fixed P\&T boundary condition at the inlet for simulation of a 150 meter fracture is quite challenging to capture correctly. 


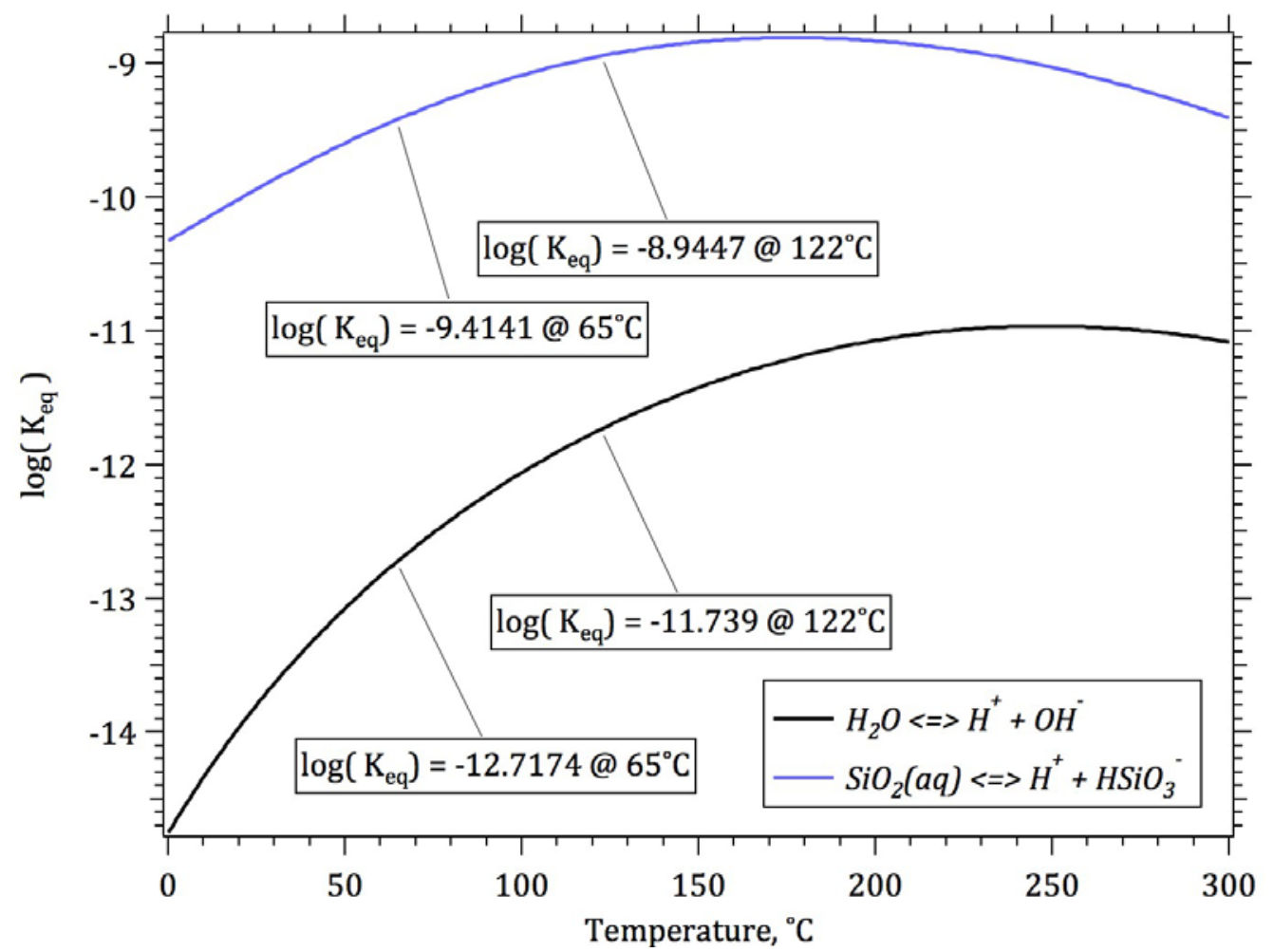

Figure 9-20. Temperature dependence of the equilibrium constants

Simulations by LBNL were performed using a relatively refined mesh, as shown in Fig. 9-21. The fracture was discretized into $0.4 \mathrm{~m}$ long blocks for a total of 375 in the X-direction. The altered granite was discretized into 375 in the X-direction and 4 in the Y-direction. The unaltered granite has gradually increasing grid block widths in the Y-direction. In total, there are 23,750 grid blocks, plus one added at the inlet to fix the boundary conditions at $X=0$. Without several sensitivity studies, it cannot be assumed to be refined enough. Even with the relatively large number of grid blocks, the observation point at 3 meters is only 8 grid blocks from the injection block. Ideally, this region should be further refined; however, this would lead to significantly longer computation times. A subdomain of this problem should be run to determine what the minimum spatial resolution is necessary to capture the "correct" results. Simulations by PNNL were performed using a computational domain with 74 x 24 (i.e., 1776 grid blocks).

Simulations by LBNL were run using TOUGHREACT V3.0-OMP (Sonnenthal et al. 2014; Xu et al. 2006; Xu et al. 2011) on a Mac Pro Intel Xeon 6-core (12-thread) CPU, using 6 threads. Numerical conditions were sequential non-iterative coupling between flow and reactive transport, with a maximum time step imposed by a CFL condition of 1 for the "pure" water case and 0.25 for the "recycle" water scenario. Simulations by PNNL were run using STOMP-CO2E (STOMP User Guide 2015; White and Oostrom 2000; White et al. 2012; White and Oostrom 2006) with ECKEChem (White and McGrail 2005) on a Mac Pro Intel Xeon 6-core (12-thread) CPU, using 1 thread. Numerical conditions were sequential non-iterative coupling between flow and reactive transport, with a maximum time step imposed by a CFL condition of 0.5 for both the "pure" and "recycle" water scenarios. 

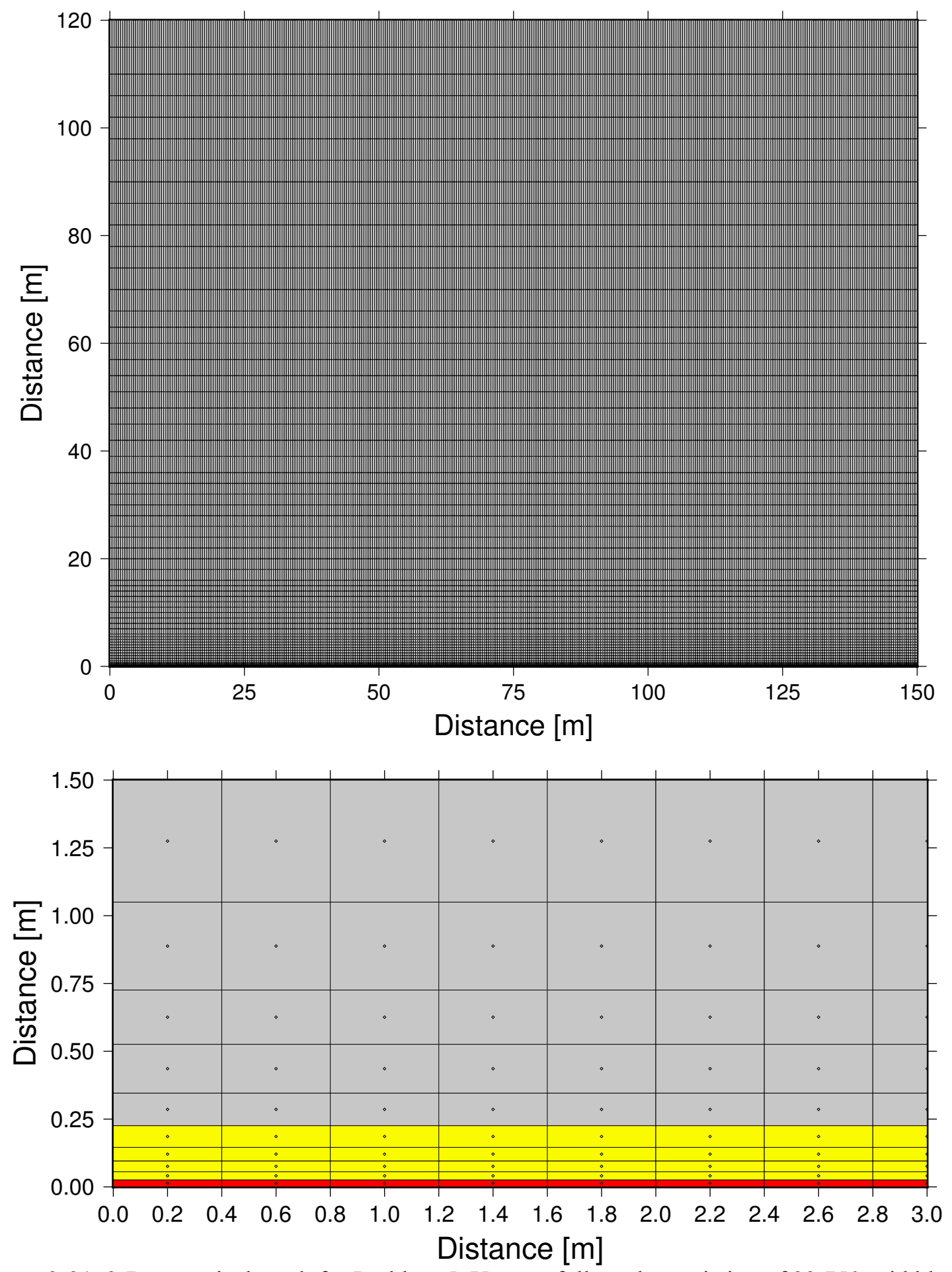

Figure 9-21. 2-D numerical mesh for Problem 5. Upper - full mesh consisting of 23,750 grid blocks $(\mathrm{NX}=375, \mathrm{NY}=62)$ for the $150 \times 120 \mathrm{~m}$ domain. Lower - enlargement of left corner $(3.0 \times 1.5 \mathrm{~m})$, showing fracture (red), altered granite (yellow), and unaltered granite (gray). The thickess in the Zdirection is $1 \mathrm{~m}$. One large volume grid block was connected to the fracture and altered granite at $\mathrm{X}=0.0$ to enforce the fixed pressure P-T-X boundary condition 



\title{
10.0 Benchmark Problem 6: Injection into a Fault/Fracture in Thermo-Poroelastic Rock
}

\author{
Problem Champion: Ahmad Ghassemi, The University of Oklahoma
}

The objective of the problem is to illustrate the role of coupled thermo-poroelastic processes resulting from water injection into a fracture/fault on the fracture's opening and shear deformation as well as on the pore pressure and stress redistributions in the neighborhood of the fracture. Both aspects have implications for stimulation and induced seismicity. The problem of fault slip and potential seismicity in response to injection in its vicinity was considered in an earlier study (Dobroskok and Ghassemi 2005) so that the focus of Benchmark Problem 6 is injection into the fracture itself with particular reference to coupled processes. For simplicity, the problem is treated in $2 \mathrm{D}$.

Two cases are considered with different injection temperatures. All deformations resulting from thermal, pore pressure, and flow are mechanical deformations and result in fracture aperture variations. No distinction is made between hydraulic aperture and mechanical aperture in these simulations. If needed, one could specify a small residual aperture for flow purposes when the fracture is mechanically closed.

Benchmark problem 6 involved injection into a fault surrounded by a poro-thermoelastic medium. Five teams participated in the solutions using various simplifying assumptions, multiple runs and THM models as listed in Table 10-1. The codes used for the simulations are described in (White et al. 2015a).

Table 10-1. Participating Teams and Simulators Used in the Solution of Benchmark Problem 6

\begin{tabular}{lll}
\hline Simulation Team & $\begin{array}{l}\text { Team } \\
\text { Identifier }\end{array}$ & Code(s) \\
\hline Pennsylvania State University & PSU & FLAC3D and TOUGHREACT \\
Stanford University & Stanford & CFRAC \\
University of Nevada, Reno & UNR & MULTIFLUX and TOUGH2 \\
The University of Oklahoma & OU & GEOFRAC \\
The University of Texas at Austin & UTA & CFRAC_UT \\
\hline
\end{tabular}

\subsection{Geometry and Input Data for Benchmark Problem 6}

Consider the 2D plane strain problem of a fracture oriented at $45^{\circ}$ in the xy plane. The fracture is $40 \mathrm{~m}$ long under the action of in-situ stresses as shown in Fig. 10-1. The rock matrix is assumed to be Westerly granite with properties shown in Table 10-2. 


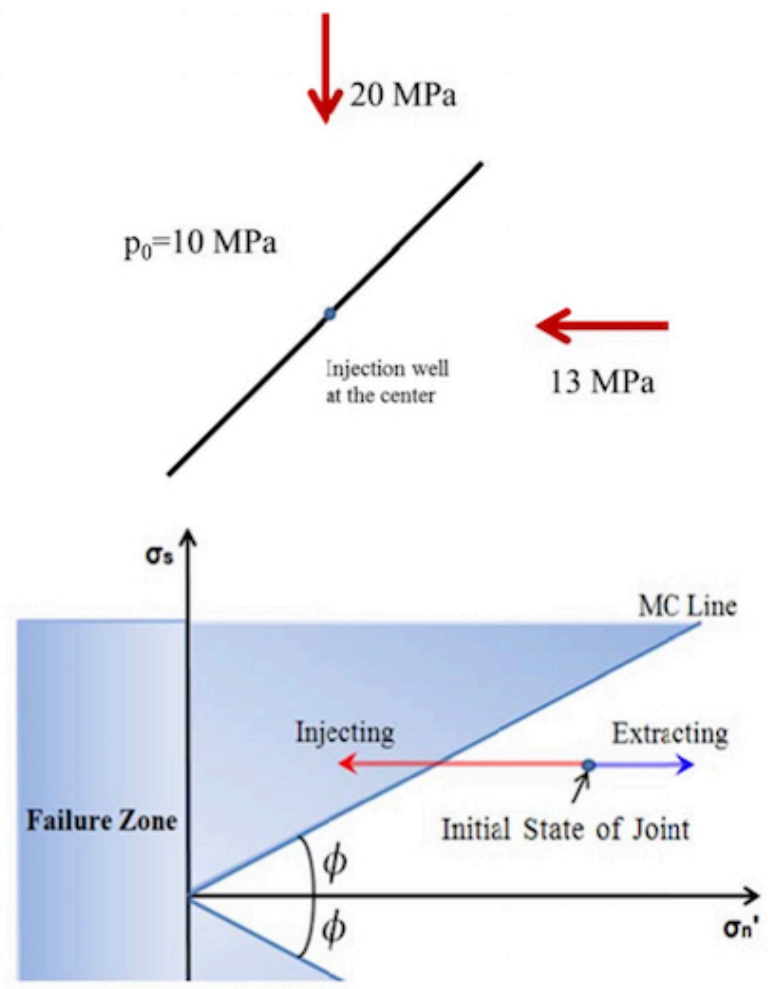

Figure 10-1. Fracture orientation and initial stress state; and Mohr-Coulomb failure diagram

Table 10-2. Material Properties

\begin{tabular}{lll}
\hline Property Name & Units & Value \\
\hline Shear modulus, G & $\mathrm{GPa}$ & 15 \\
Poisson's ratio, $v$ & -- & 0.25 \\
Undrained Poisson's ratio, $v_{u}$ & -- & 0.33 \\
Matrix permeability, $k$ & $\mathrm{~m}^{2}$ & $4 \times 10^{-19}$ \\
Matrix porosity, $\phi$ & -- & 0.01 \\
Biot's coefficient, $\alpha$ & -- & 0.44 \\
Water Viscosity, $\mu_{w}$ & & $2.037 \times 10^{-4}$ at $400^{\circ}$ \\
& $\mathrm{Pa} \mathrm{sec}$ & $3.547 \times 10^{-4}$ at $320^{\circ}$ \\
Fluid compressibility, $C_{w}$ & $1 / \mathrm{MPa}$ & $4.2 \times 10^{-4}$ \\
Thermal expansion coefficient of solid, $\alpha_{\mathrm{s}}$ & $1 / \mathrm{K}$ & $2.4 \times 10^{-5}$ \\
Thermal expansion coefficient of fluid, $\alpha_{\mathrm{f}}$ & $1 / \mathrm{K}$ & $2.1 \times 10^{-4}$ \\
Thermal diffusivity of intact porous rock, $\mathrm{c}^{\mathrm{T}}$ & $\mathrm{m} / \mathrm{s}$ & $1.1 \times 10^{-6}$ \\
Fluid density, $\rho_{w}$ & $\mathrm{~kg} / \mathrm{m}^{3}$ & $1 \times 10^{3}$ \\
Heat capacity of fluid, $c_{w}$ & $\mathrm{~J} \mathrm{~kg} \mathrm{~K}^{-1}$ & 4200 \\
Initial joint normal stiffness, $k_{n}$ & $\mathrm{GPa} / \mathrm{m}^{3}$ & 0.5 \\
Initial joint shear stiffness, $k_{s}$ & $\mathrm{GPa} / \mathrm{m}$ & 50 \\
Maximum joint closure, $D_{c m a x}$ & $\mathrm{~mm}$ & 3.0 \\
Initial fracture aperture, $D_{n i}$ & $\mathrm{~mm}$ & 1.0 \\
In-situ stress in the y-direction, $\sigma_{\xi}$ & $\mathrm{MPa}$ & 20 \\
In-situ stress in the x-direction, $\sigma_{\psi}$ & $\mathrm{MPa}$ & 13 \\
Friction angle (effective), cohesion, $\varphi$ & $\mathrm{MPa}$ & 30 \\
\hline
\end{tabular}




\subsection{Initial and Boundary Conditions and Sources for Benchmark Problem 6}

The initial pore pressure is $10 \mathrm{MPa}$ and the rock/fracture system is at a temperature of $420^{\circ} \mathrm{K}$. The joint is in equilibrium with the prescribed stress and pore pressure and temperature conditions, and has an aperture of $0.5623 \mathrm{~mm}$. This is the actual joint aperture under reservoir conditions and is calculated internally using the prescribed initial stiffness and stress state. The maximum joint closure is $3 \mathrm{~mm}$.

Two cases are considered: 1) injection water temperature of $420^{\circ} \mathrm{K}$ (isothermal case) and 2) injection water temperature of $320^{\circ} \mathrm{K}$ (non-isothermal case). Injection begins at time $\mathrm{t}=0$ and is specified at a constant rate of $0.6 \times 10^{-7} \mathrm{~m}^{3} / \mathrm{s}$ per meter thickness of reservoir.

Liquid viscosity of the injected fluid is chosen to be at the average system temperature. To isolate the impact of thermal stresses, the water viscosity is unchanged when comparing isothermal (injection water has the same temperature as the reservoir rock) and non-isothermal (injection water has a different temperature than the reservoir rock) injection cases.

The problem does not consider the possibility of natural fracture deformation under initial in-situ stress and pore pressure fields. Variations of density or viscosity with temperature are neglected during simulations.

\subsection{Solution Metrics for Benchmark Problem 6}

1. Pressure, fracture opening/closure, and shear deformation as a function of time at the center of the fracture for both cases (0-180 days)

2. Fracture opening and shear, at 72 and 180 days (isothermal case)

3. Fracture opening at 5 and 180 days (non-isothermal case)

\subsection{Results for Benchmark Problem 6}

Because the physics of the problem was not constrained and some teams included poroelastic stresses in the matrix rock while others did not, the results are presented separately for each set of assumptions. While OU and PSU considered poroelastic stresses in their simulations, UTA and UNR did not include this feature in their solutions. Stanford submitted results for both assumptions and both cases (isothermal and non-isothermal). Fig. 10-2 illustrates the pressure with injection time for the isothermal case where poroelastic stresses are considered. While the curves have similar shapes, the Stanford model predicts a slightly higher pressure. The ISO 13528 uncertainty is shown in Fig. 10-3, illustrating an uncertainty of only $1 \mathrm{MPa}$ for this case, less than $10 \%$.

For the isothermal case in which only pressurization of the fracture was considered, the results for pressure versus time are also in good agreement (Fig. 10-4) with the ISO 13528 uncertainty is $\sim 5-10 \%$ (Fig. 10-5). Note that the UNR results were not included in the ISO uncertainty calculation because they did not continue fir the entire simulation time period. 


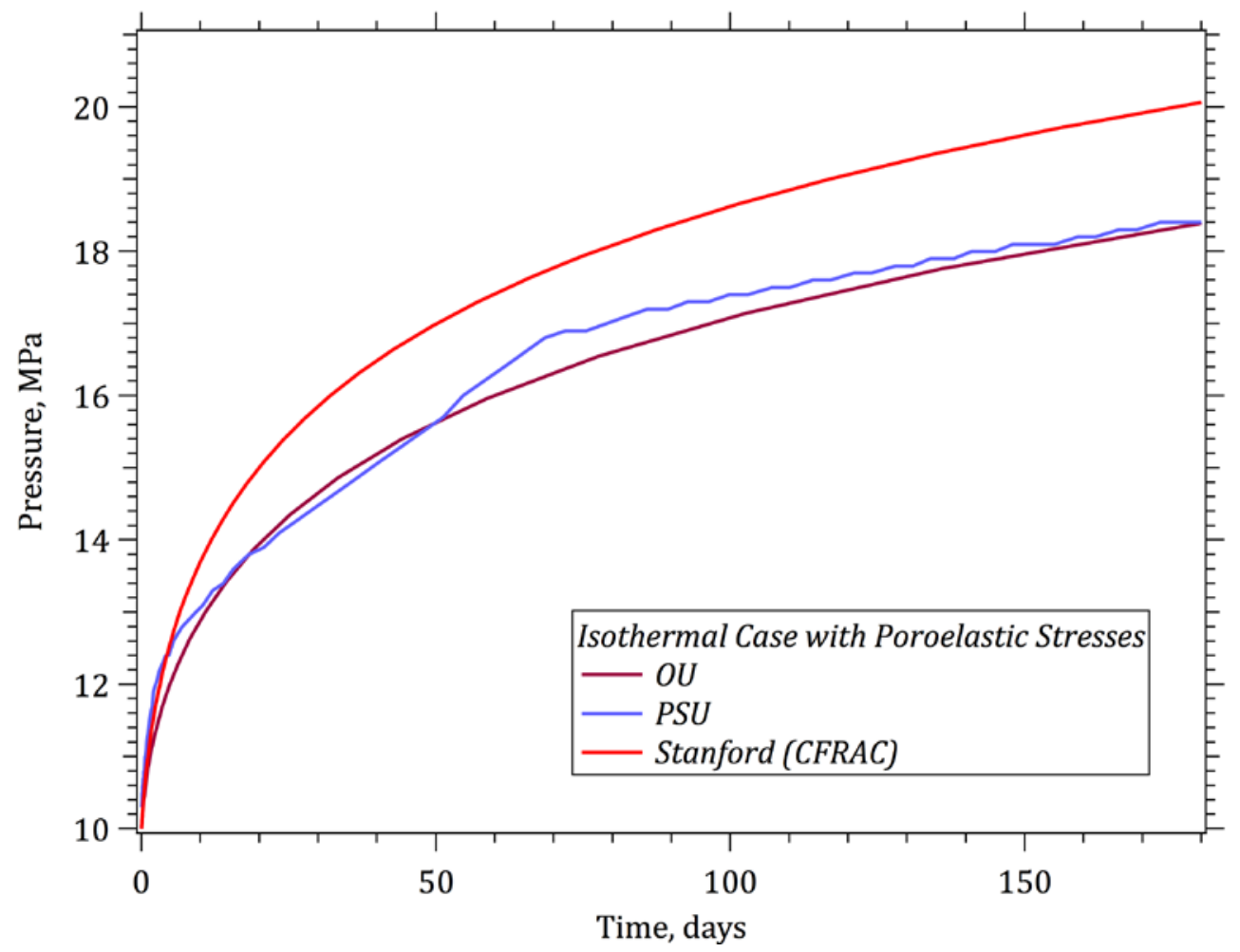

Figure 10-2. Simulation results for pressure versus time for the isothermal case in which poroelastic stresses in the matrix rock are considered

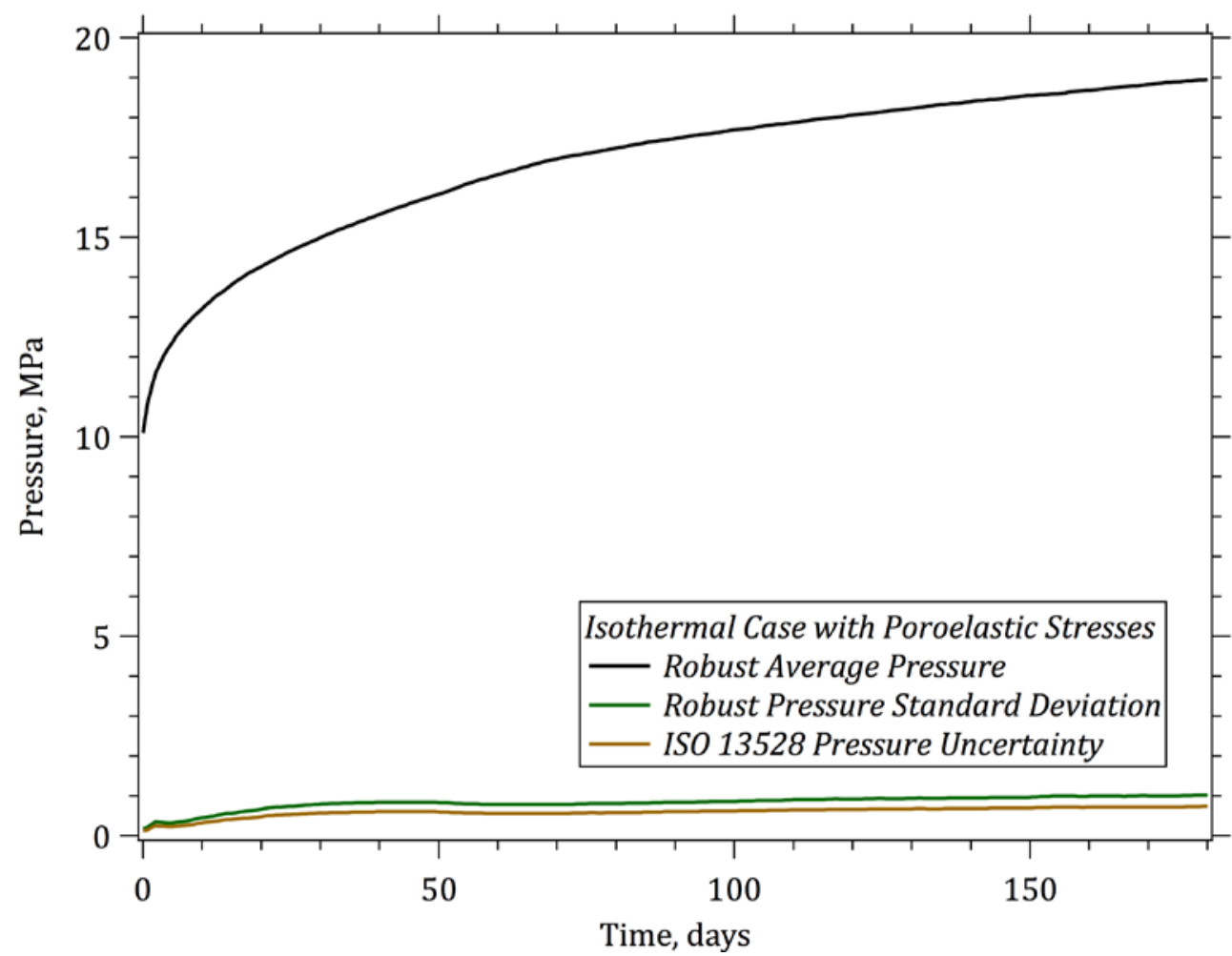

Figure 10-3. ISO 13528 uncertainty for pressure versus time for the isothermal case in which poroelastic stresses in the matrix rock are considered 


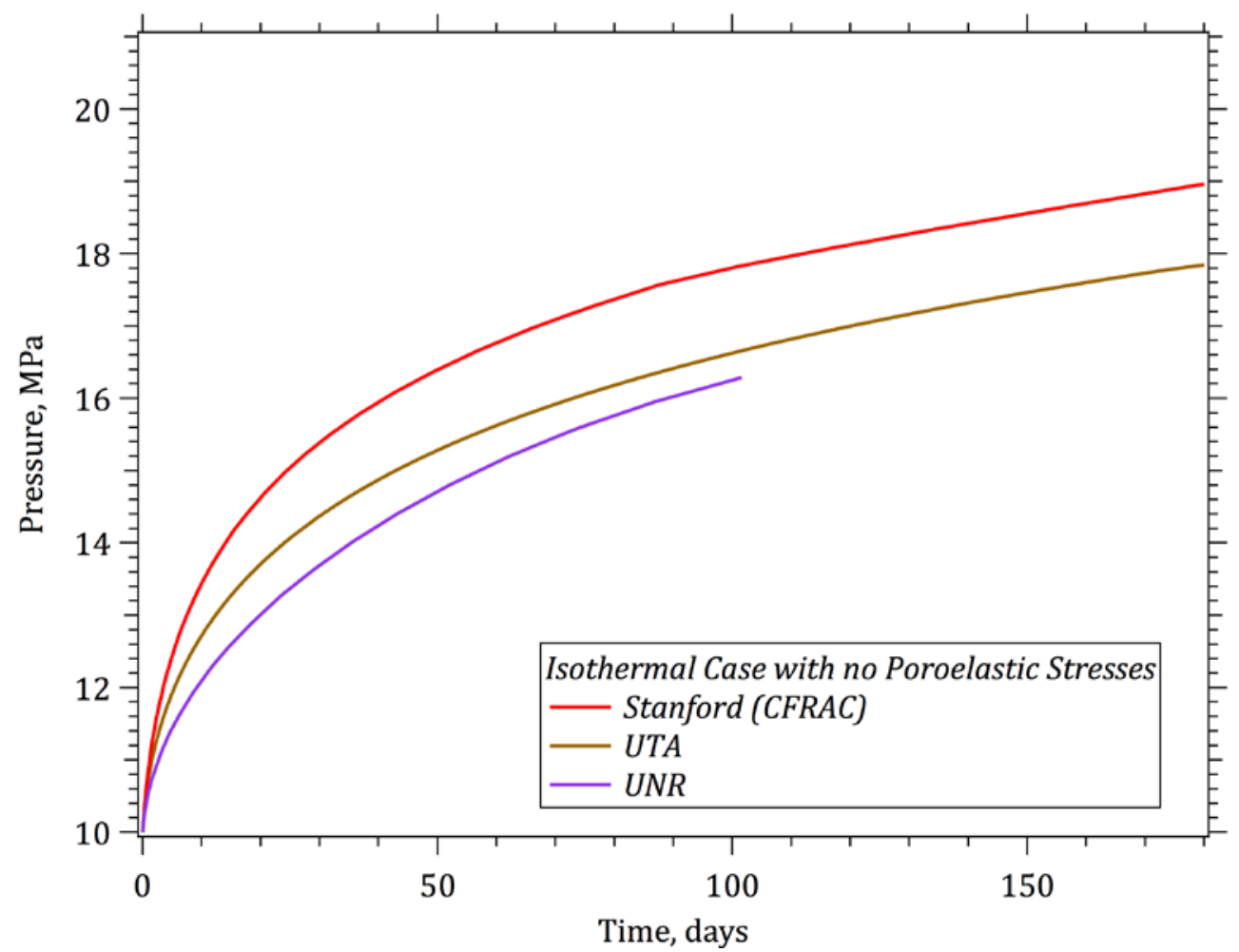

Figure 10-4. Simulation results for pressure versus time for the isothermal case in which poroelastic stresses in the matrix rock are not considered.

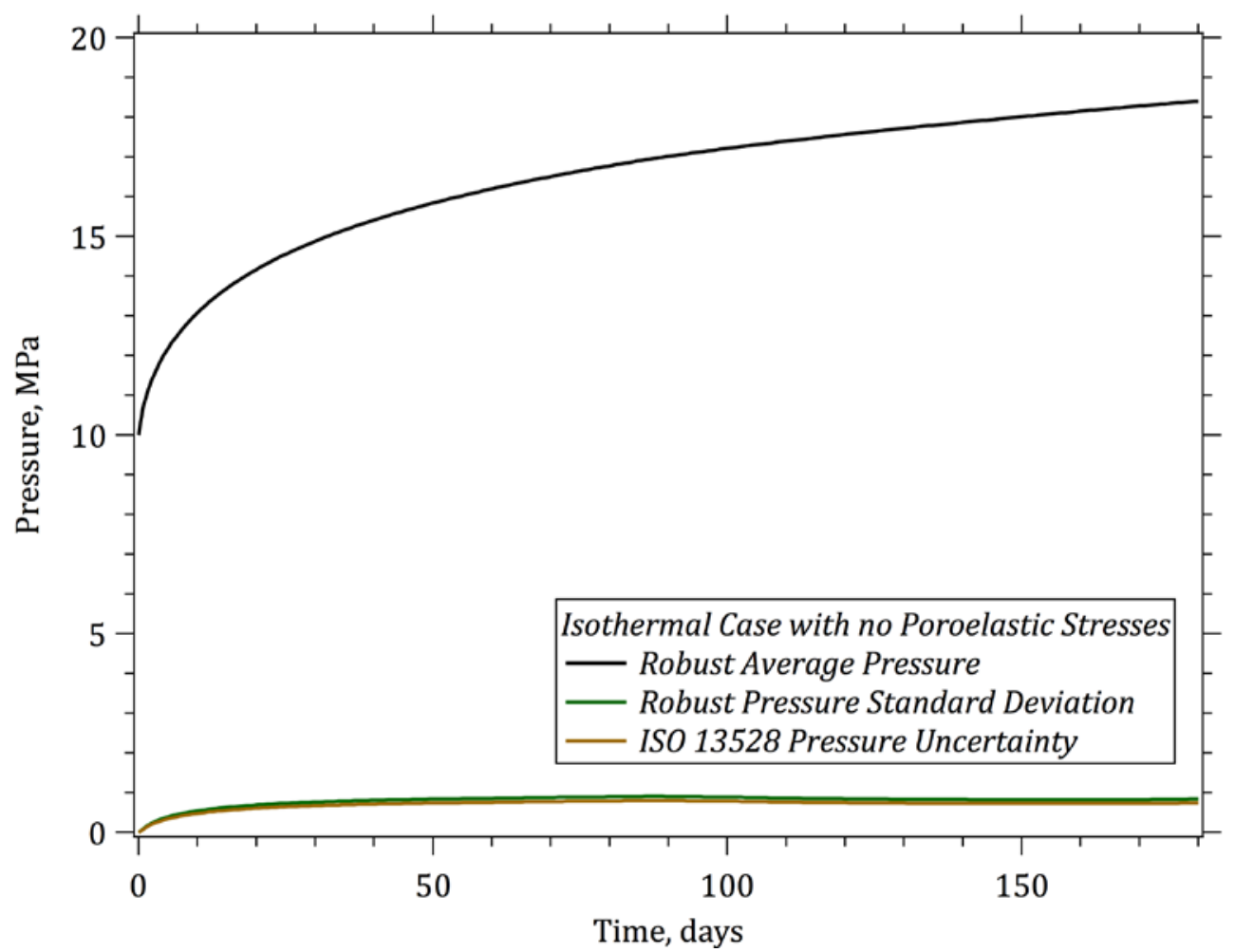

Figure 10-5. ISO 13528 uncertainty for pressure versus time for the isothermal case in which poroelastic stresses in the matrix rock are not considered 
Aperture variation at the center of the fracture with injection time is shown in Fig. 10-6 for the isothermal case with poroelastic stresses, in Fig. 10-7 for the isothermal case without poroelastic stresses, and in Fig. 10-8 for the non-isothermal case with poroelastic stresses. Note that cooling tends to increase the fracture aperture; however, in view of the low temperature contrast (DT) between the injection water and the rocks and the low value of thermal diffusivity, thermal stress is not very large and develops very slowly. The thermal stress effect is focused in the central region of the fracture where most cooling occurs, and it expands with time. Increasing DT increases the cooling-induced crack opening. Note also the contrast between the profiles for the isothermal and cooling cases. It should be emphasized that cooling in the crack is controlled by the residence time of the fluid which is influenced by injection rate and leak-off, and fracture permeability.

Figs. 10-9 to 10-11 show the shear deformation along the fracture length 72 days into the simulation and at the end of the simulation (180 days). Simulation results compare quite favorably for the isothermal case in which poroelastic streses are considered (Fig. 10-9). For the isothermal case in which poroelastic streses are not considered, the OU and Stanford results differ in early time, but converge to nearly the same solution by the end of the simulation (Fig. 10-10). The non-isothermal case also shows the same pattern of timing (Fig. 10-11).

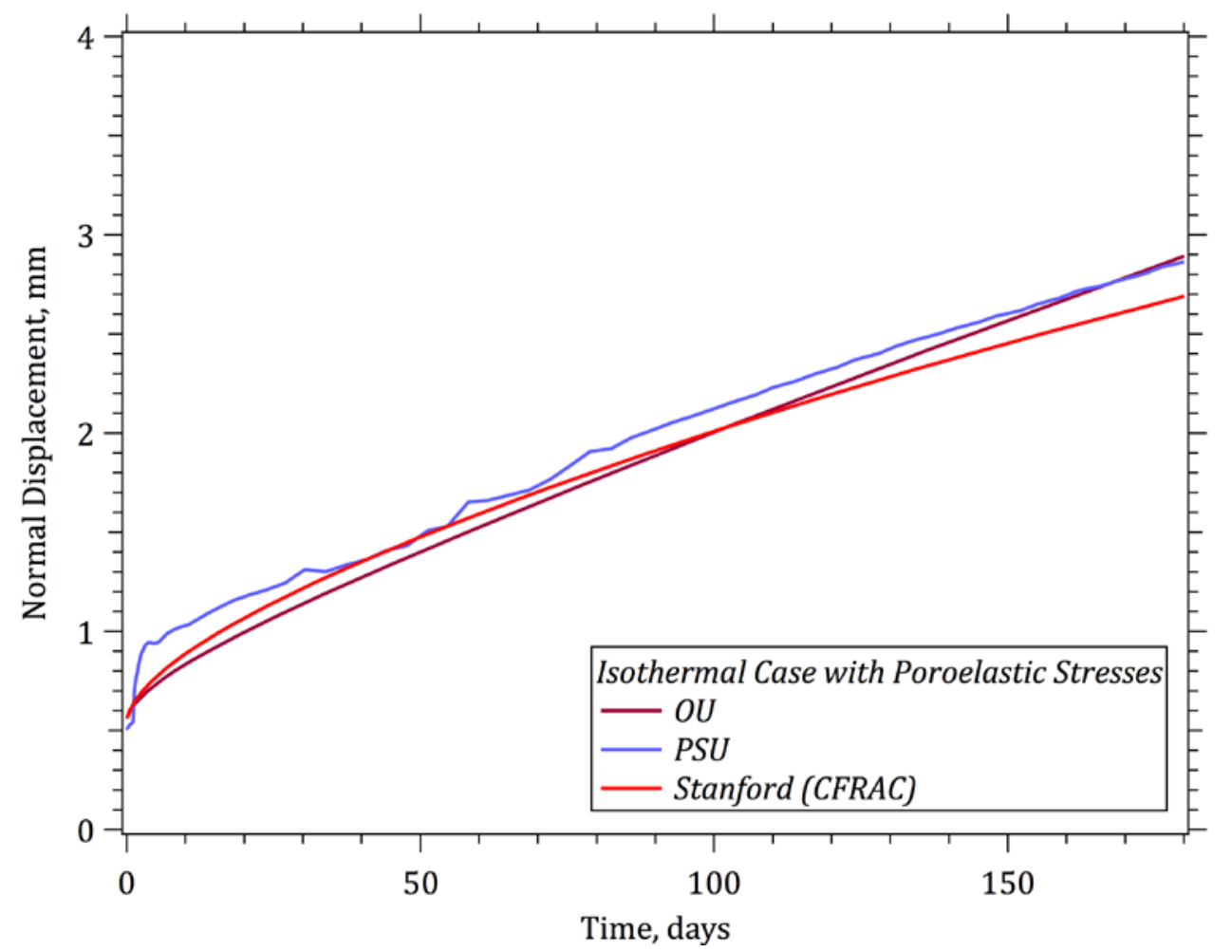

Figure 10-6. Simulation results for fracture opening versus time for the isothermal case in which poroelastic stresses in the matrix rock are considered 


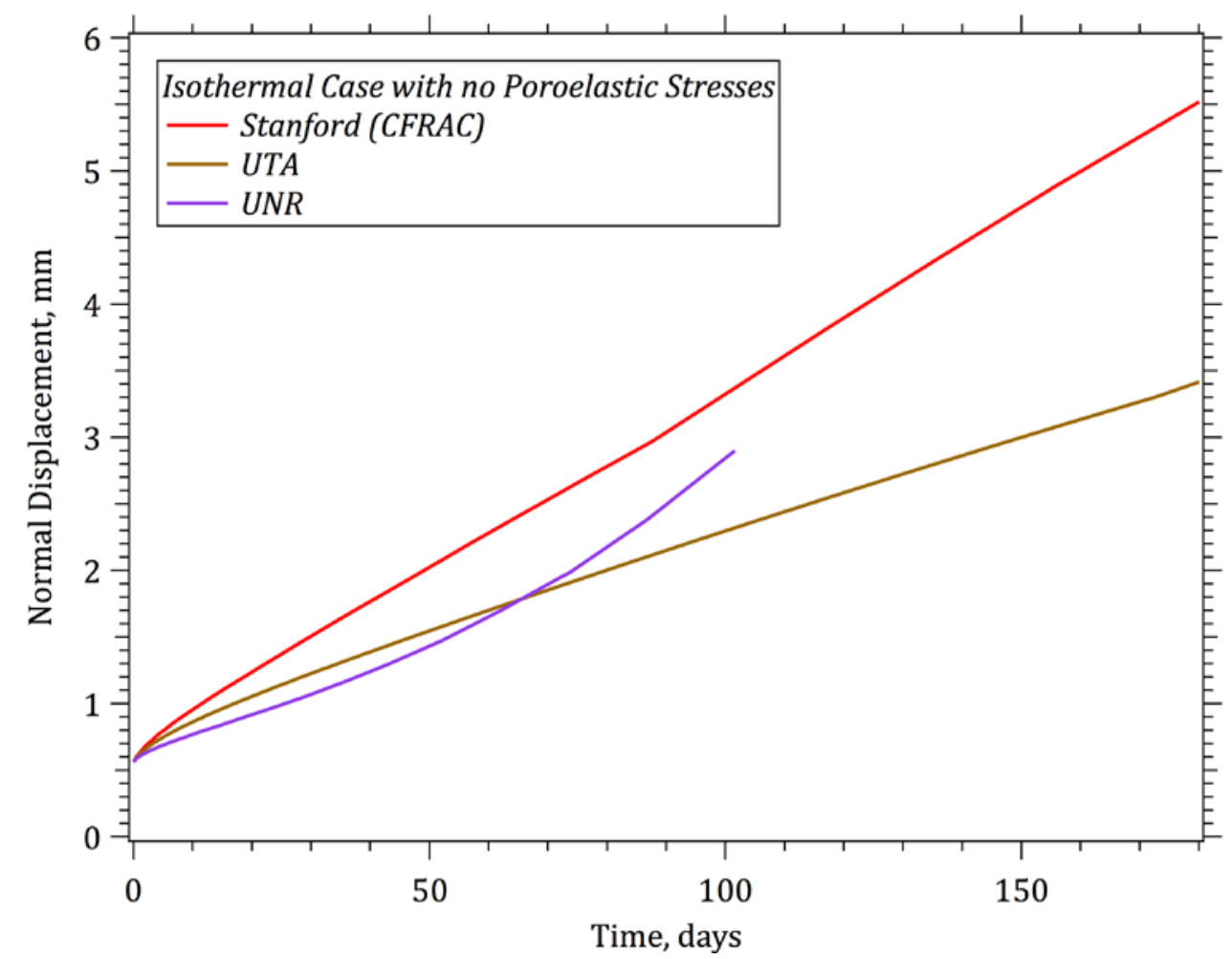

Figure 10-7. Simulation results for fracture opening versus time for the isothermal case in which poroelastic stresses in the matrix rock are not considered

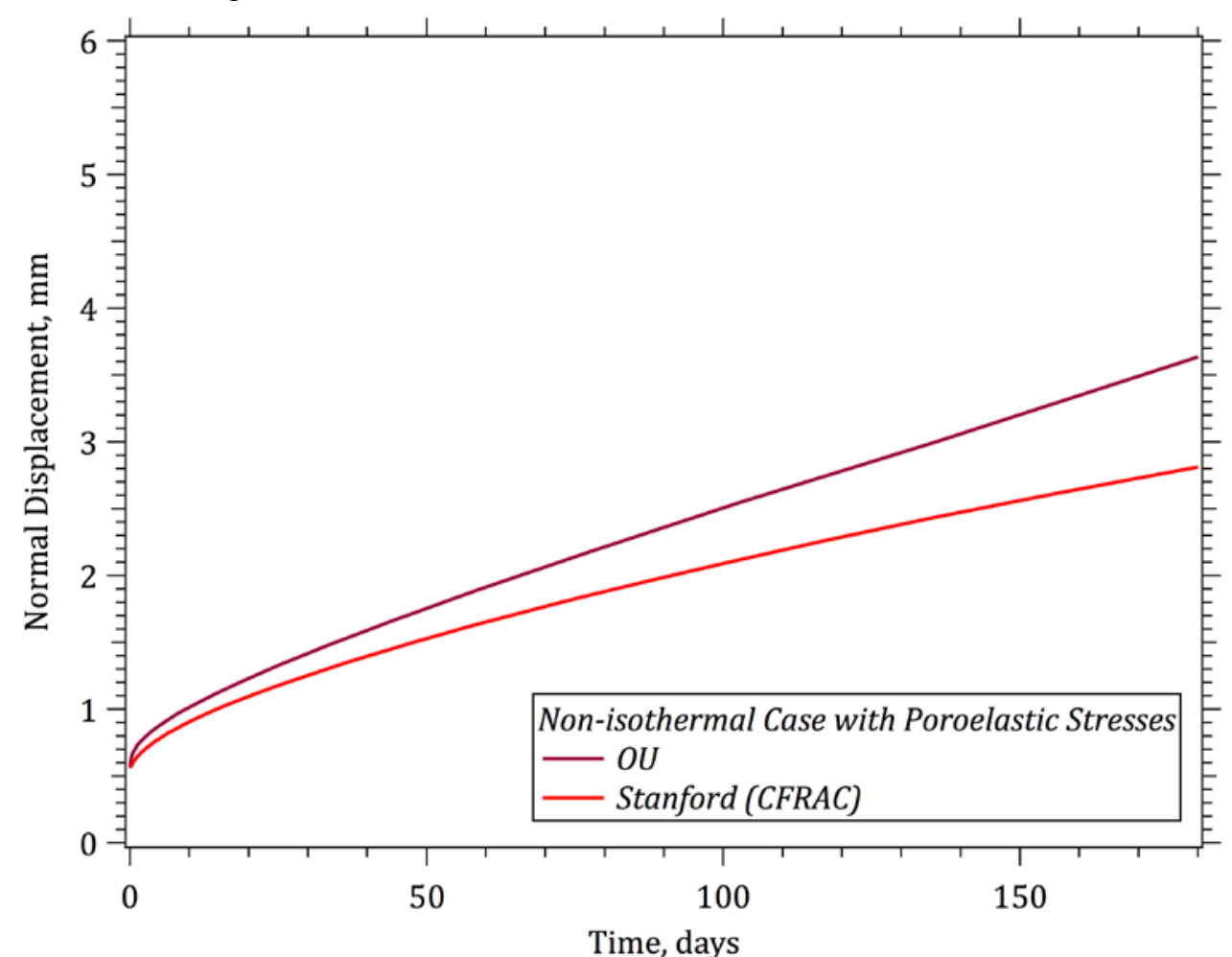

Figure 10-8. Simulation results for fracture opening versus time for the non-isothermal case in which poroelastic stresses in the matrix rock are considered 


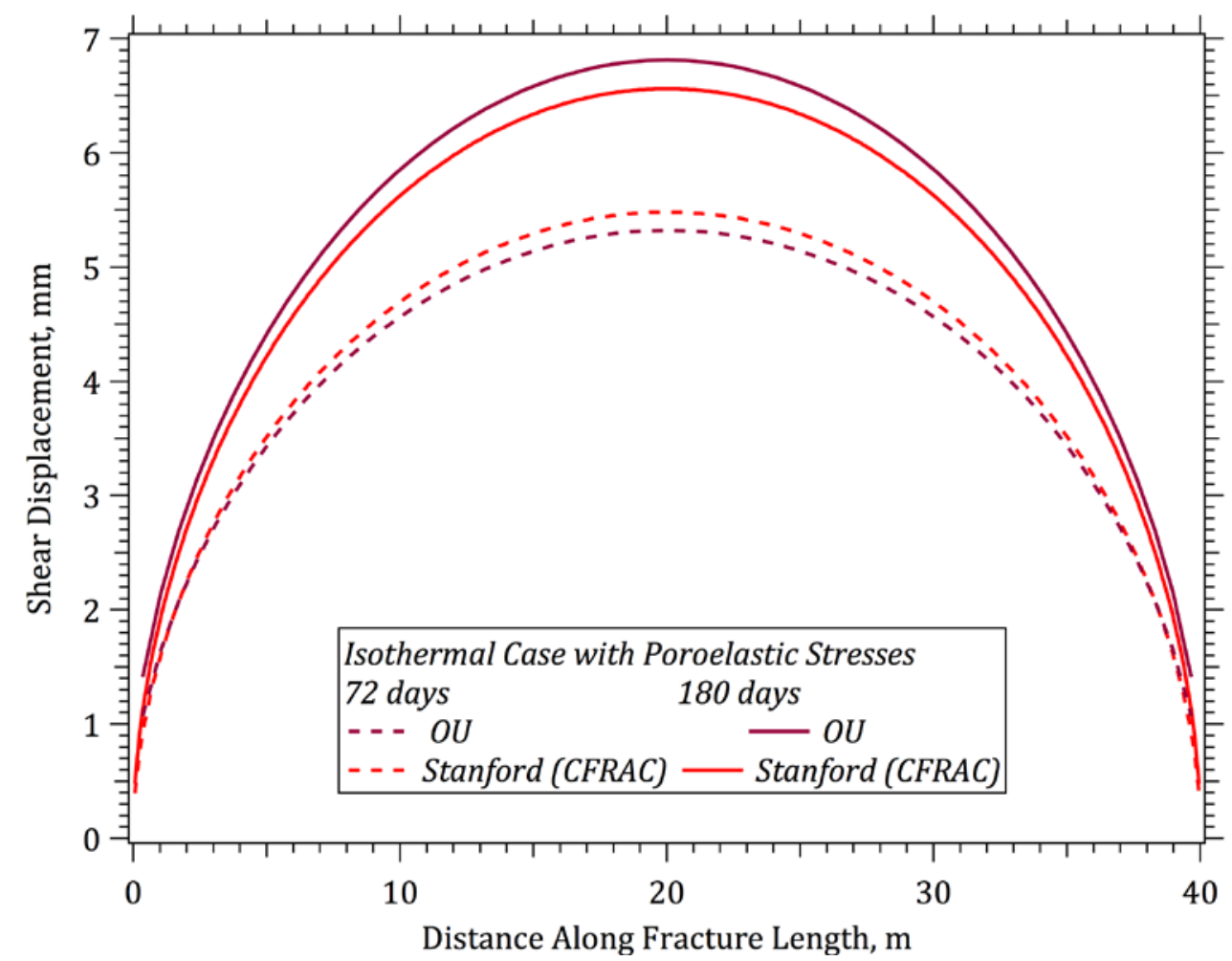

Figure 10-9. Simulation results for shear deformation along the fracture length at 72 days and 180 days for the isothermal case in which poroelastic stresses in the matrix rock are considered

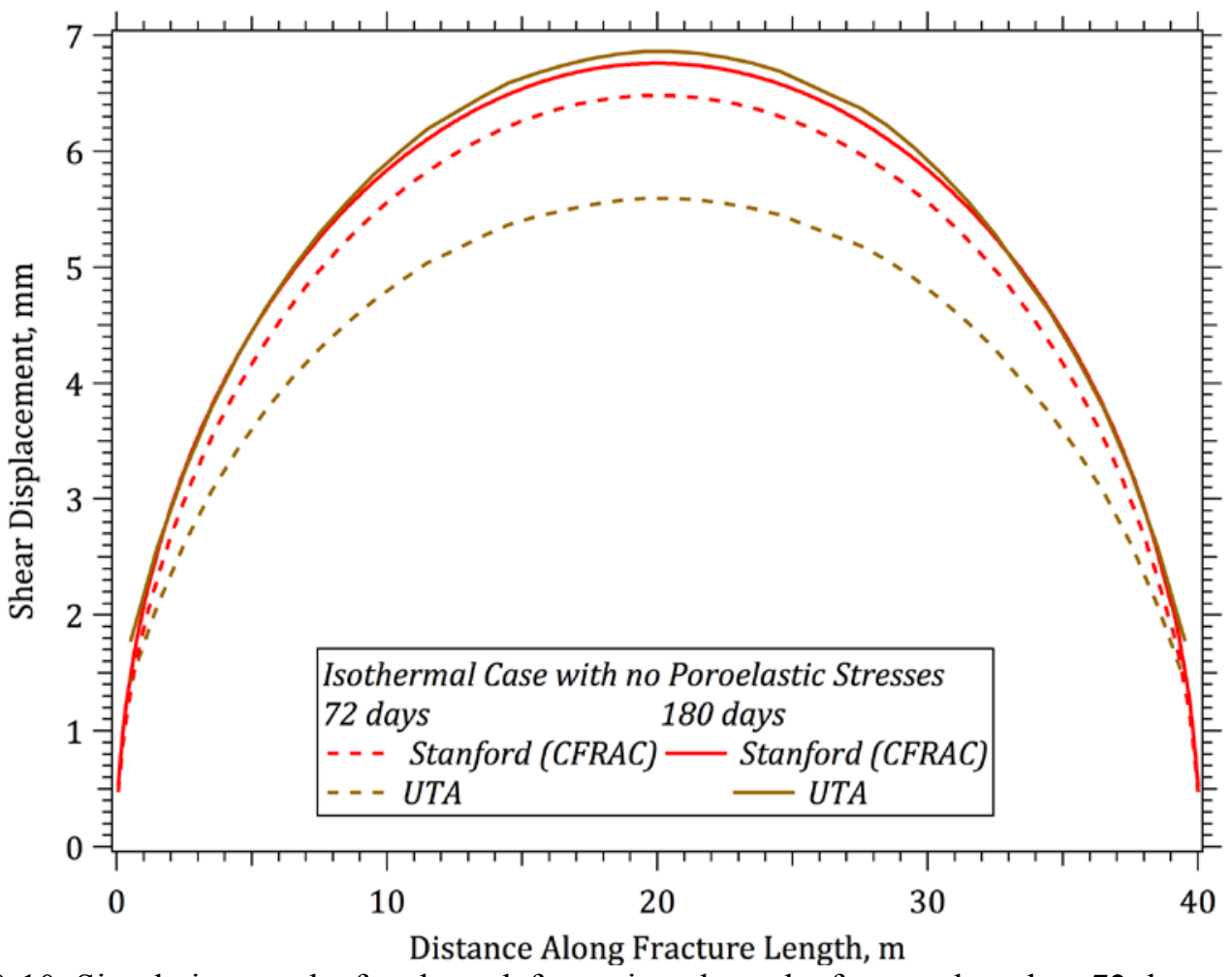

Figure 10-10. Simulation results for shear deformation along the fracture length at 72 days and 180 days for the isothermal case in which poroelastic stresses in the matrix rock are not considered 


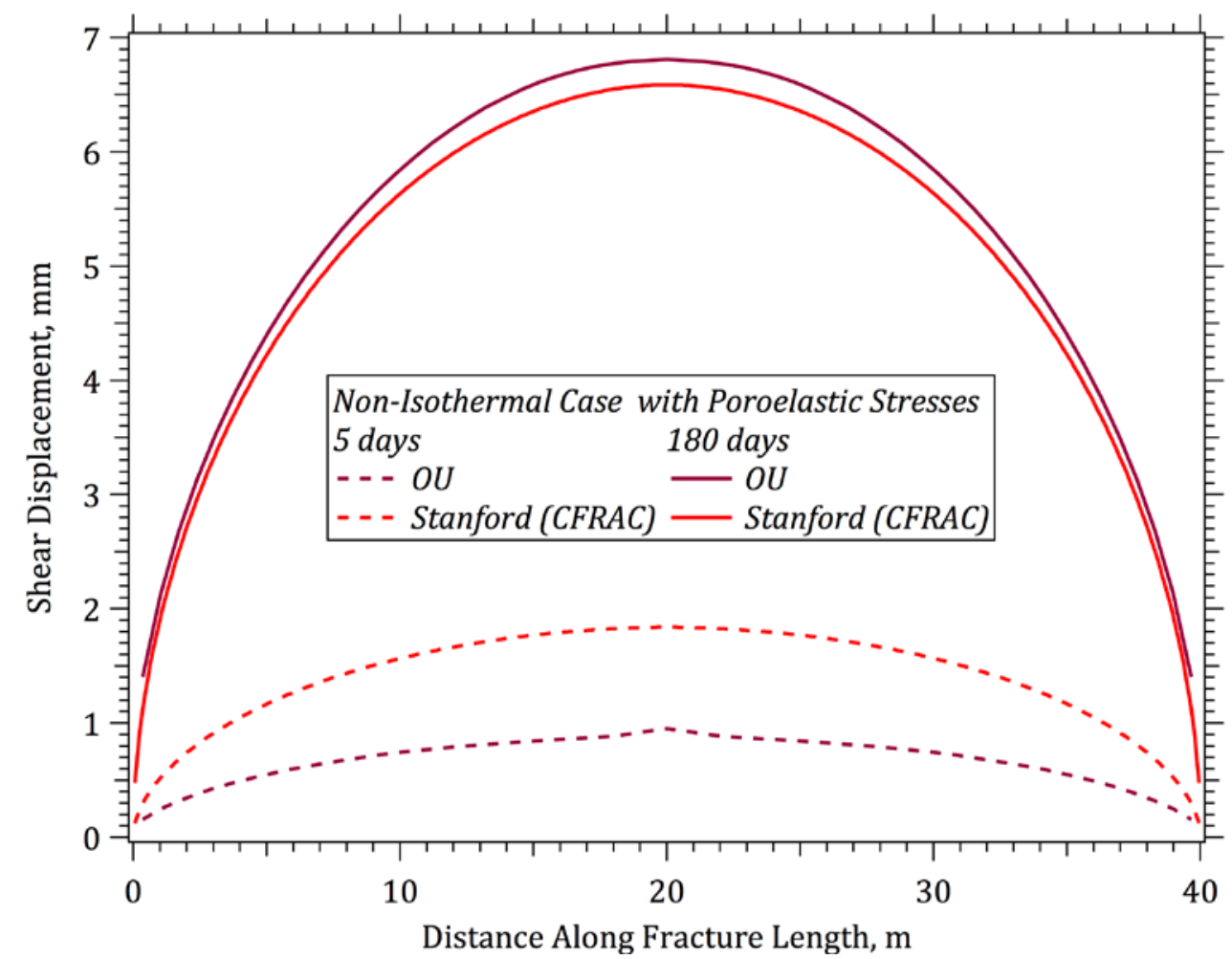

Figure 10-11. Simulation results for shear deformation along the fracture length at 5 days and 180 days for the non-isothermal case in which poroelastic stresses in the matrix rock are considered

Simulation results for fracture opening along the length of the fracture are shown in Figs. 10-12 to 10-14. In this case, the OU and Stanford models show better agreement in early time for the isothermal case in which poroelastic streses are considered (Fig. 10-12). The results for the isothermal case in which poroelastic streses are not considered, show a greater disparity in model results, with the UTA model showing much less growth in the fracture opening (Fig. 10-13). In the non-isothermal case, the thermal stress effect is focused in the central region of the fracture where most cooling occurs, and it expands with time. This can be seen in Fig. 10-14 in both the OU and Stanford results. The less pronounced effect in the Stanford results is likely due to differences in grid resolution. 


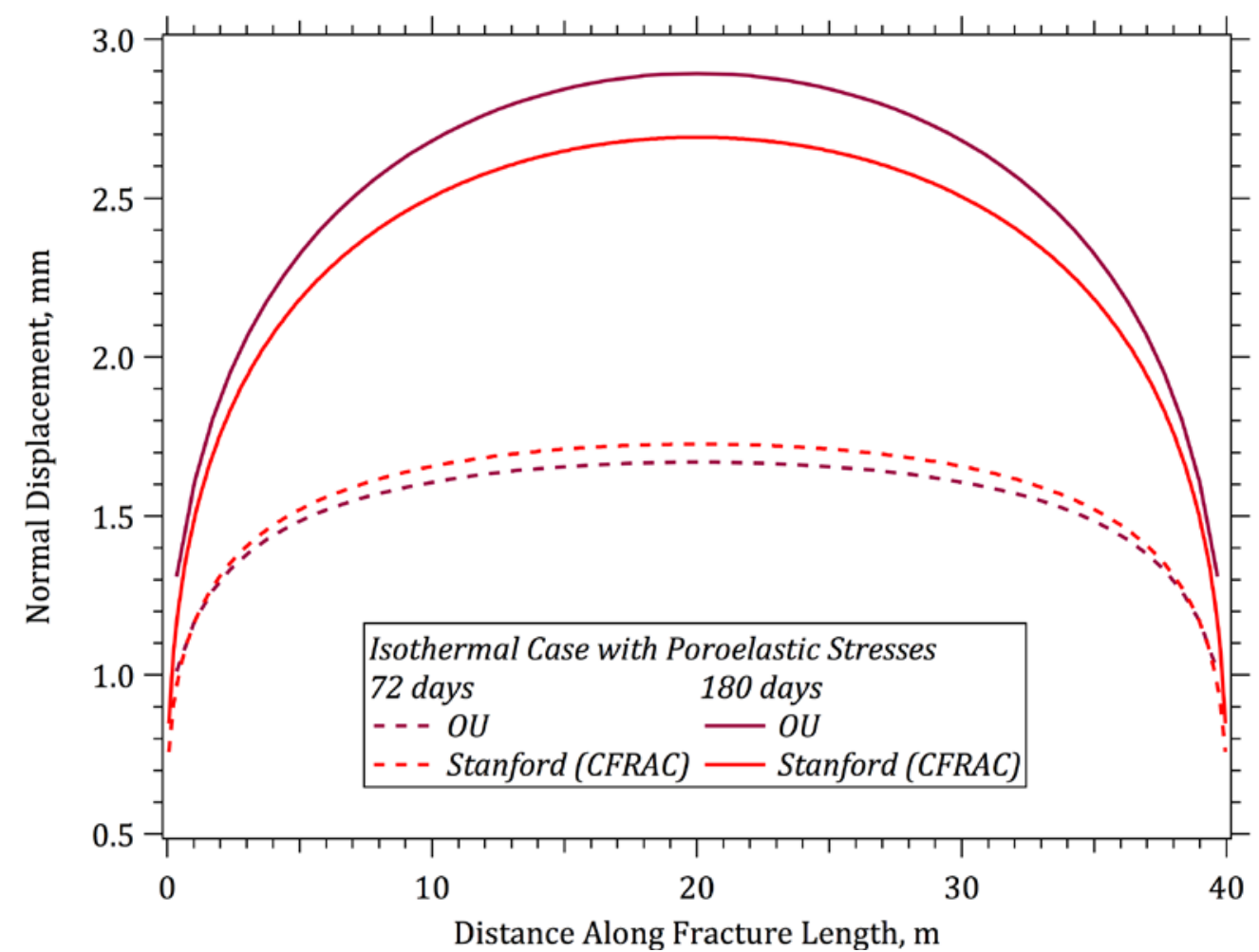

Figure 10-12. Simulation results for fracture opening along the fracture length at 72 days and 180 days for the non-isothermal case in which poroelastic stresses in the matrix rock are considered

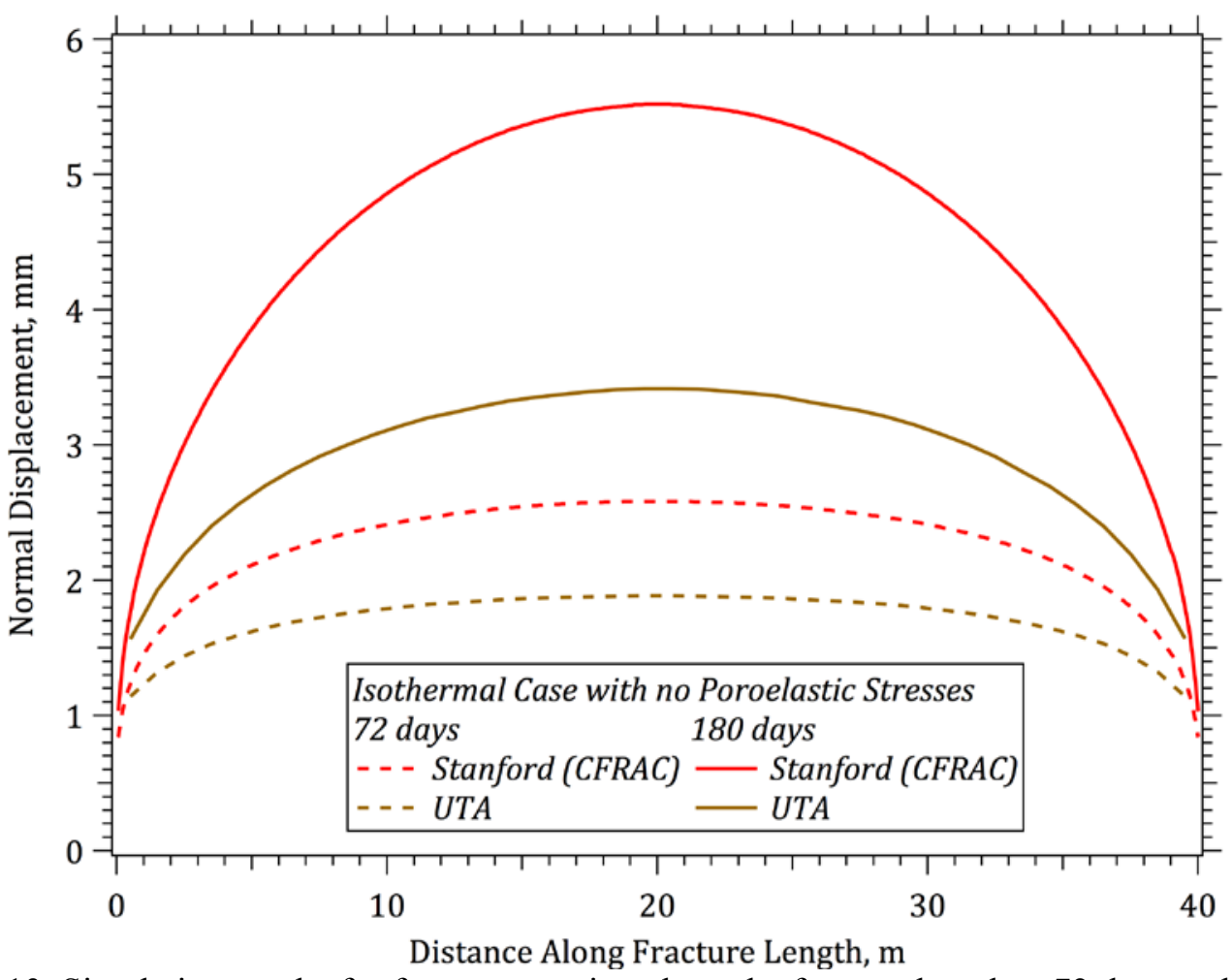

Figure 10-13. Simulation results for fracture opening along the fracture length at 72 days and 180 days for the isothermal case in which poroelastic stresses in the matrix rock are not considered 


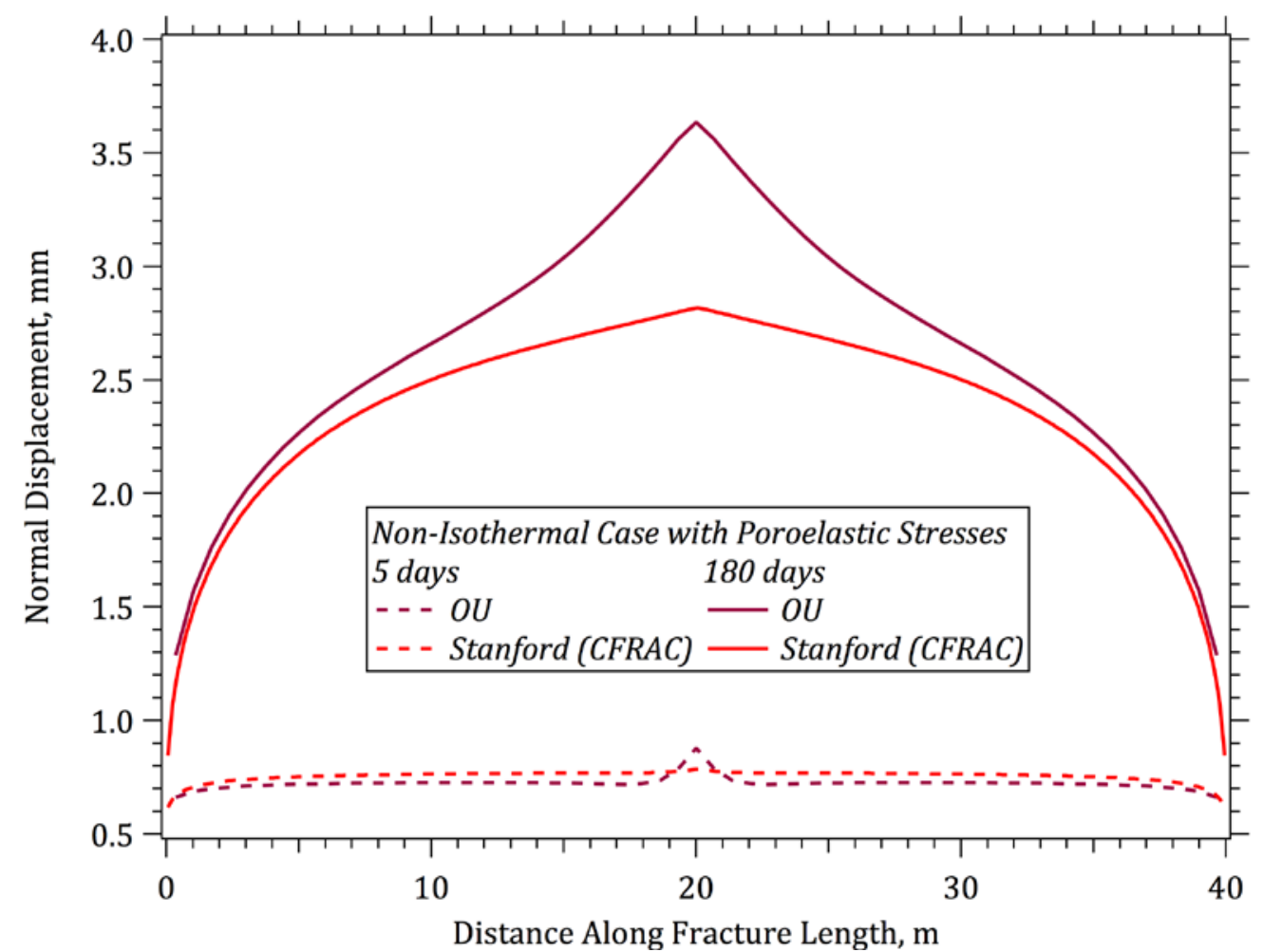

Figure 10-14. Simulation results for fracture opening along the fracture length at 5 days and 180 days for the non-isothermal case in which poroelastic stresses in the matrix rock are considered

\subsection{Discussion of Benchmark Problem 6 Results}

The submitted solutions are in general agreement. Variation can be attributed to various simplifying assumptions of the models and solution procedures. To differing degrees, the solutions submitted are affected by the following factors: mesh refinement, coupling level in the constitutive equations (uncoupled poroelasticity, lack of coupling between temperature and pore pressure), leak-off into the matrix and its dependence on pressure, 1D or 2D calculations of induced poroelastic and thermoelastic stresses, inclusion of stresses caused by joint deformation (these can play a role in this problem and can contribute to the timing of joint element separation which impact flow). These influencing factors would also impact pore pressure and stress distributions around the fracture. 



\title{
11.0 Benchmark Problem 7: Surface Deformation from a Pressurized Subsurface Fracture
}

\author{
Problem Champion: Pengcheng Fu, Lawrence Livermore National Laboratory
}

Quantifying ground surface deformation caused by the hydraulic stimulation of subsurface reservoir is an important means for understanding reservoir characteristics and reservoir behavior. InSAR

(Interferometric synthetic aperture radar) and tiltmeter measurements are widely used for this purpose. For reservoirs dominated by discrete fractures and stimulations that create discrete fractures, surface deformation measurements can be particularly useful in identifying the stimulated fractures and estimating their dimensions. For instance, InSAR data collected at the In Salah $\mathrm{CO}_{2}$ storage site have evidenced the creation of hydraulic fractures in the cap rocks by the injection (White et al. 2014a). The ability to predict ground surface signatures in conjunction with modeling various responses of subsurface reservoirs is a much-desired feature for reservoir stimulation codes because it enables direct model validation against field observables. Benchmark Problem 7 in the GTO code comparison program aims to demonstrate and compare various codes' abilities to predict ground surface deformations caused by the pressurization of a subsurface fracture.

This problem entails the calculation of ground surface deformation caused by a pressurized subsurface fracture. The solid medium is assumed to be isotropic and homogeneous and linearly elastic. The effects of the pressurized fluid are represented by a uniform pressure applied onto the two fracture walls. The fracture is assumed to be rectangular in shape and various dipping angles are considered. In addition to the full 3D solution, the problem is reduced to a plane-strain geometry, so that 2D codes can participate in the comparison and results can be compared with those available in the literature. Due to the relatively slow transient processes associated with fluid and heat flow compared with the transient processes in the solid phase (namely, wave propagation), we only consider the pseudo-static solution of this problem.

Based on a literature survey, the problem design is loosely based on the plane-strain solution of (Pollard and Holzhausen 1979), in which numerical solutions of the surface deformation caused by the pressurization of a subsurface fracture as well as the stress intensity factors at fracture tips are provided. A search of comparable 3D solutions did not yield directly useful results. Although earth surface deformation caused by the displacement of faults is often considered in geology, analytical solutions in that area (e.g. (Okada 1985)) typically assume known displacement fields along the fault, which is inappropriate for the applications considered here.

Six teams participated in the solutions using various simplifying assumptions, multiple runs and HM models as listed in Table 11-1. The codes used for the simulations are described in (White et al. 2015a).

Table 11-1. Participating Teams and Simulators Used in the Solution of Benchmark Problem 7

\begin{tabular}{lll}
\hline Simulation Team & Team Identifier & Code(s) \\
\hline Idaho National Laboratory & INL & FALCON \\
Itasca Consulting Group & Itasca & FLAC \\
Lawrence Livermore National Laboratory & LLNL & GEOS \\
Pennsylvania State University & PSU & FLAC \\
The University of Oklahoma & OU TOUGHREACT \\
The University of Texas at Austin & UTA & GEOFRAC \\
\hline
\end{tabular}




\subsection{Geometry and Input Data for Benchmark Problem 7}

We first present the 3D geometry. As illustrated in Figure 11-1, the rectangular fracture is $2 a$ wide and $2 b$ long with a dipping angle $\beta$. A global coordinate system and a local coordinate system are created. The origin of the global $\mathrm{x}-\mathrm{y}-\mathrm{z}$ coordinate system is at the projection of the fracture center on the ground surface. The $y$-axis is along the vertical direction pointing upwards and the $z$-axis is along the strike direction. The origin of the local $u-v$ coordinate system is at the fracture center. The u-axis is along the strike or the length direction while the v-axis is along dipping or the width direction. The fracture center is at a depth of $d$ so the $\mathrm{u}-\mathrm{v}$ coordinate system's origin $(u=0, v=0)$ has a coordinate $(0,-d, 0)$ in the global coordinate system. If $b>>a$ and $b>>d$, this $3 \mathrm{D}$ geometry can be modeled as a 2D plane-strain problem. The 2D geometry is essentially a vertical cut of the 3D model in the $\mathrm{x}-\mathrm{y}$ plane. Note that the coordinate system is problem-dependent and each problem in the present paper establishes different coordinate systems.

If in situ stress is concerned, the pressure applied on the fracture surfaces $\left(p_{0}\right)$ should be considered the "net pressure", which is the difference between the fluid pressure and the in situ normal stress acting on the fracture plane. Anisotropy of in situ stress will not affect the results if the fracture happens to be horizontal or vertical $\left(\beta=0\right.$ or $\left.90^{\circ}\right)$, but will affect the results for oblique fractures (e.g. $\beta=45^{\circ}$ ) due to the shear stress on fracture faces. Therefore, the setup of the problem implies isotropic in situ stress.

The geometrical and material parameters are given in Table 11-2. All results are presented in a nondimensionalized form, with units only given to add some engineering reference to the problem.

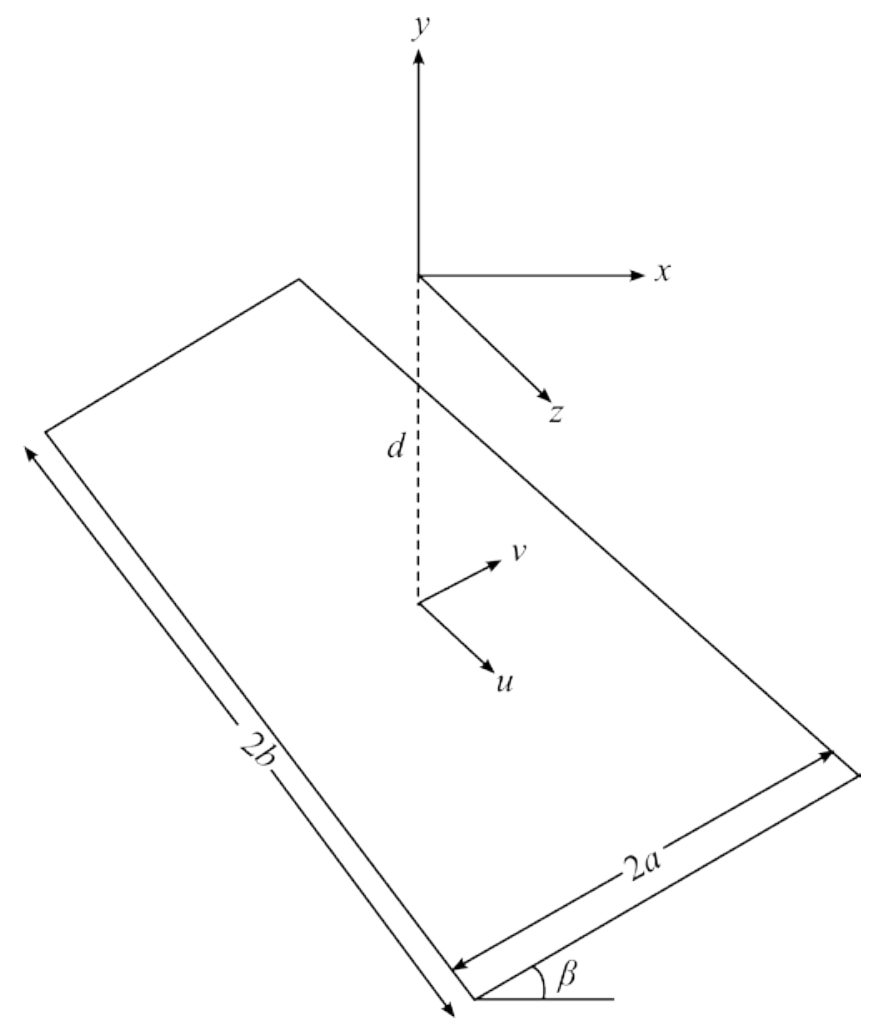

Figure 11-1. Geometry of the fracture in 3D and the coordinate systems 
Table 11-2. Material Property Table

\begin{tabular}{lll}
\hline Property Name & Units & Value \\
\hline$a$ & $\mathrm{~m}$ & 100 \\
$b$ & $\mathrm{~m}$ & 300 \\
$d$ & $\mathrm{~m}$ & 125 \\
$\beta$ & $\mathrm{deg}$ & $0,45,90$ \\
Young's Modulus, $E$ & $\mathrm{GPa}$ & 10.0 \\
Poisson's ratio, $v$ & -- & 0.25 \\
Net fluid pressure, $p_{0}$ & $\mathrm{MPa}$ & 1.0 \\
\hline
\end{tabular}

\subsection{Initial and Boundary Conditions and Sources for Benchmark Problem 7}

This problem assumes an equilibrium state and has no specified initial condition or state. The "reference configuration" for deformation quantification is the configuration under any given and fixed in situ stress with a zero net-pressure along the fracture. Although no boundary conditions other than the pressure inside the fracture are specified, because this problem concerns the deformation of an infinite half space, the simulation domain must be sufficiently large so that the boundary condition applied at the far field has negligible effects on the near-field responses. A sensitivity analysis in (Bahrami et al. 2015) found that as long as the dimensions of the computational domain are more than 10 times larger than those of the fracture, the results are insensitive to the choice of the model size and far-field boundary conditions.

\subsection{Solution Metrics for Benchmark Problem 7}

The primary observables to be compared are the vertical surface displacement $\delta_{y}$ over the pressurized fracture. In 2D, $\delta_{y}$ from $x=-4 a$ to $x=4 a$ is to be reported. Following the convention in (Pollard and Holzhausen 1979), $\delta_{y}$ should be normalized by $\delta_{\infty}=2 P_{0} a\left(1-v^{2}\right) / E$, which is the maximum normal displacement of the walls of a 2D fracture in an infinite elastic domain. Results for $\beta=0^{\circ}$ and $90^{\circ}$ can be compared with those in Fig. 8 of (Pollard and Holzhausen 1979). For the 3D model, $\delta_{y}$ along the $x$-axis from $x=-4 a$ to $x=4 a$ and $\delta_{y}$ along the $x$-axis from $z=-4 b$ to $z=4 b$ should be reported.

We are also interested in the codes' ability to predict stress intensity factors (SIF) along the tips of the pressurized fractures. For the 2D model, the mode-I and mode-II stress intensity factors at the two fracture tips $(v= \pm a)$ should be reported and reference values are available in (Pollard and Holzhausen 1979). We term the tip closer to ground surface tip A and the other tip B for easy differentiation. For 3D, the mode-I and mode-II stress intensity factors along the fracture edge with $v=a$ (the long edge closer to ground surface) were invited to be reported. All SIF values should be normalized by $K_{0}=P_{0}(\pi a)^{0.5}$.

\subsection{Results for the surface displacement in 2D}

Results submitted by the five teams for the 2D problem are shown in Figs. 11-2 to 11-4 for the three dipping angles $\beta=0^{\circ}, 45^{\circ}$, and $90^{\circ}$, respectively. The calculated robust averages, robust standard deviations, and ISO 13528 uncertainties are also shown. Overall the results match each other very well. Particularly, the results submitted by INL, Itasca, and LLNL are almost identical to each other and to the results in (Pollard and Holzhausen 1979) for all three dipping angles. The results of OU over-predict the median predicted surface deformation and those of PSU seem to slightly under-predict. The differences 
are relatively small (within 7\%) but the trend of over-/under-prediction is very consistent among all dipping angles simulated. Because the differences between the OU/PSU results and the rest are not only in the overall vertical locations of the curves, but also in the vertical distances between the highest and lowest points along the curves, they could not have been caused by the difference in domain sizes used by different teams. They likely reflect differences in the formulations and solution methods of the codes or meshing densities and configurations.

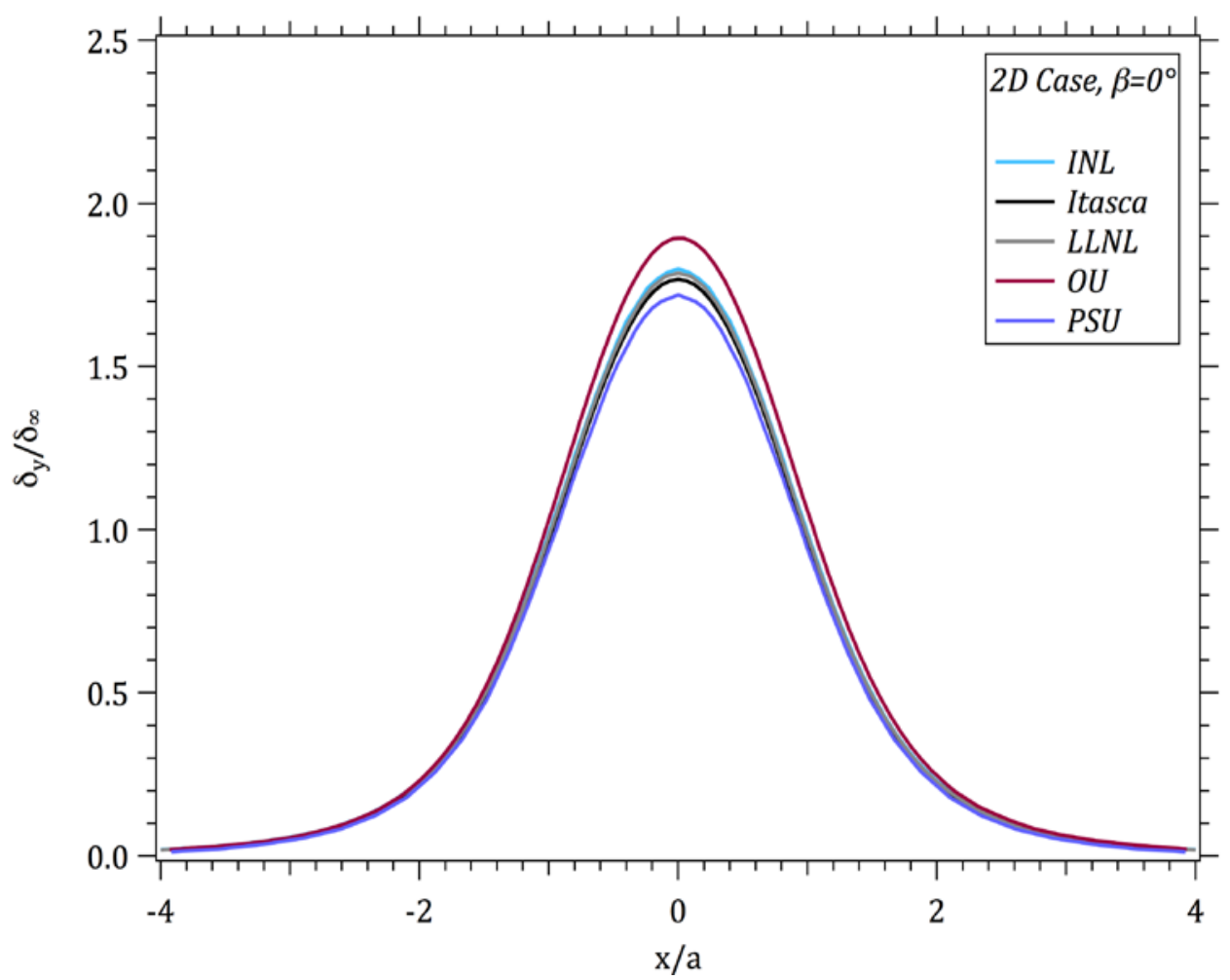

Figure 11-2. Vertical surface displacement $\delta_{y}$ normalized by $\delta_{\infty}$ from $\mathrm{x}=-4 \mathrm{a}$ to $4 \mathrm{a}$ for $\beta=0^{\circ}$ for the $2 \mathrm{D}$ case 


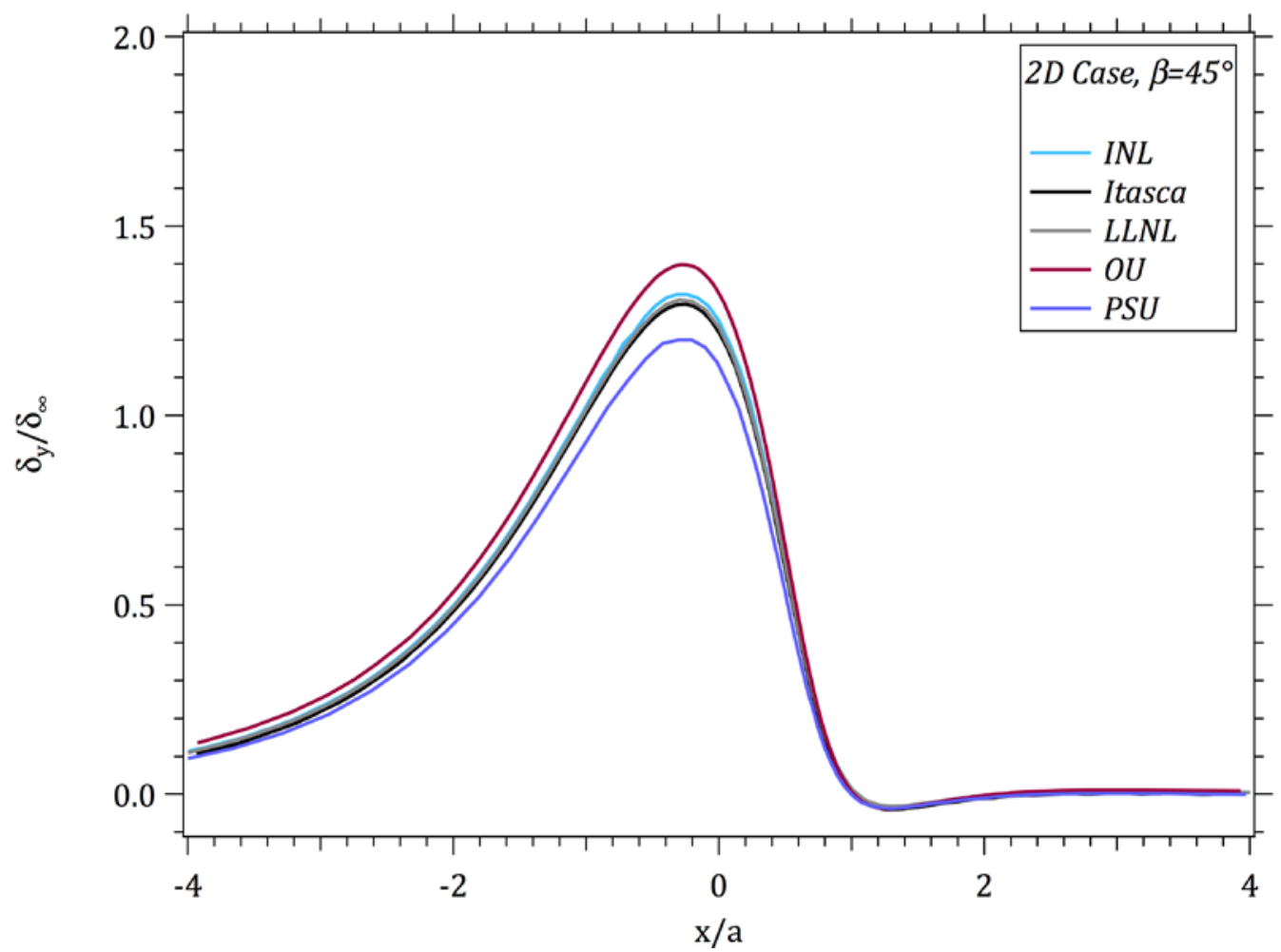

Figure 11-3. Vertical surface displacement $\delta_{y}$ normalized by $\delta_{\infty}$ from $\mathrm{x}=-4 \mathrm{a}$ to $4 \mathrm{a}$ for $\beta=45^{\circ}$ for the $2 \mathrm{D}$ case

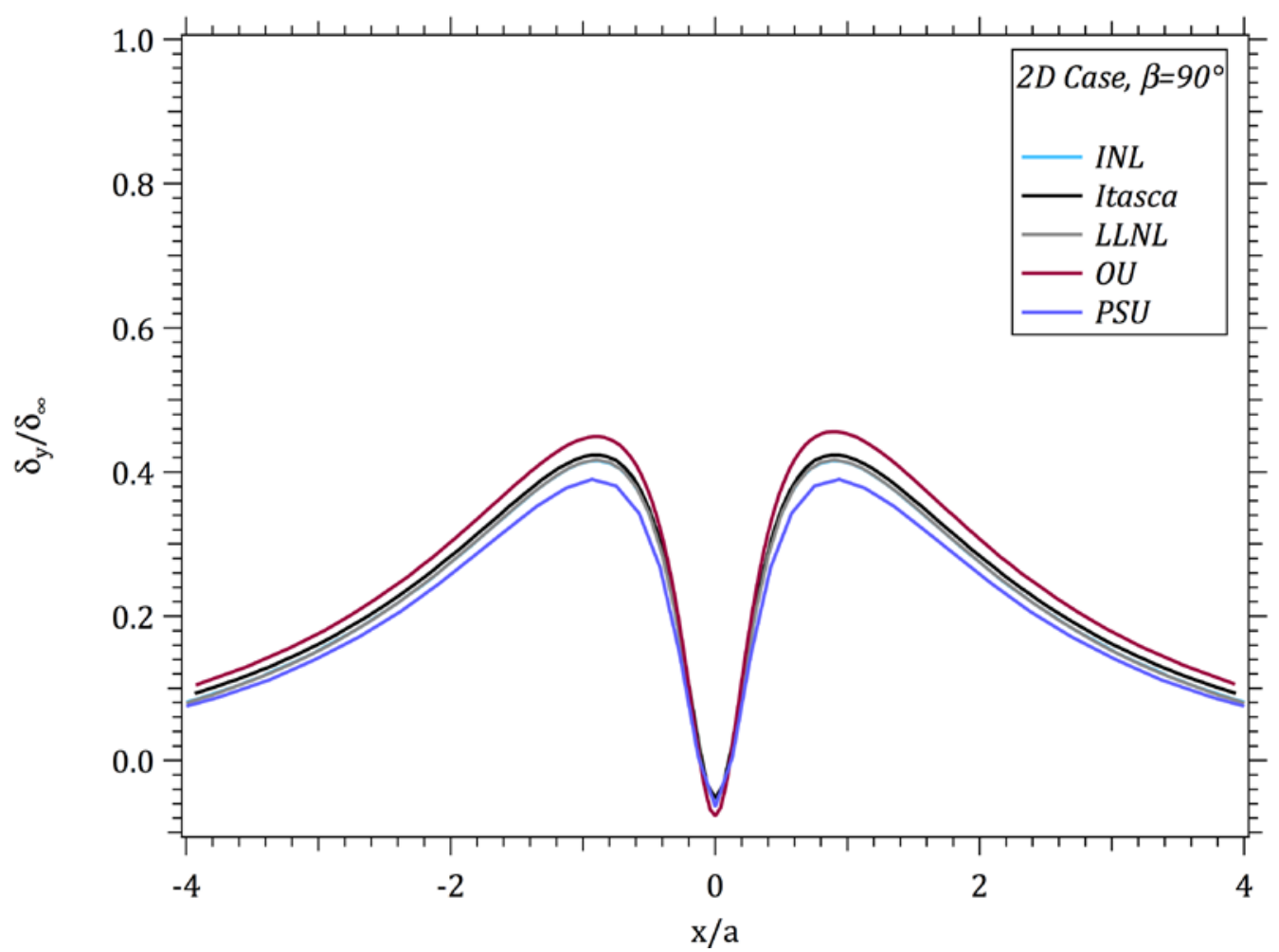

Figure 11-4. Vertical surface displacement $\delta_{y}$ normalized by $\delta_{\infty}$ from $\mathrm{x}=-4 \mathrm{a}$ to $4 \mathrm{a}$ for $\beta=90^{\circ}$ for the $2 \mathrm{D}$ case 


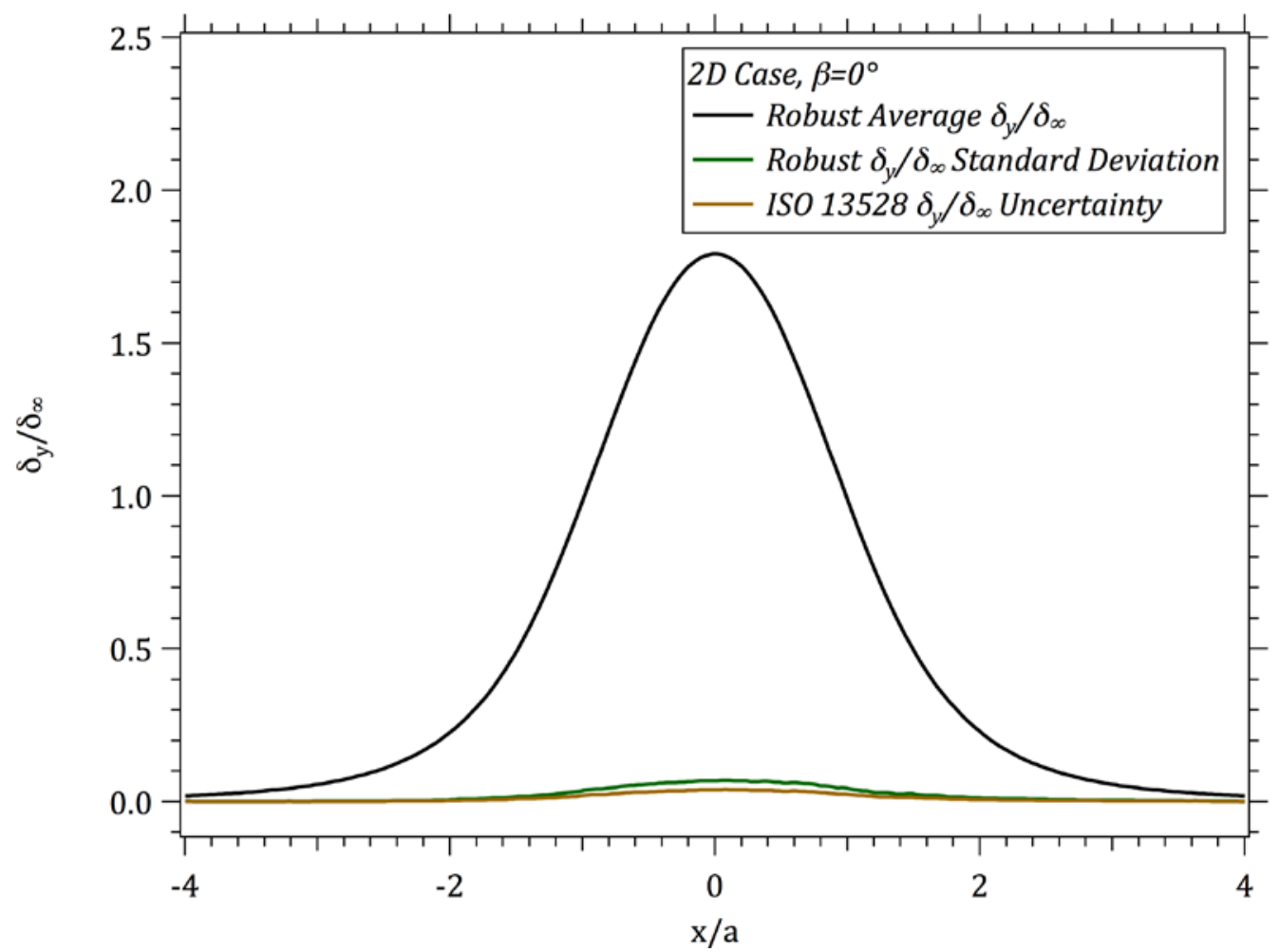

Figure 11-5. Vertical surface displacement $\delta_{y}$ normalized by $\delta_{\infty}$ from $\mathrm{x}=-4$ a to $4 \mathrm{a}$ for $\beta=90^{\circ}$ for the $2 \mathrm{D}$ case

\subsection{Results for the surface displacement in 3D}

Four teams submitted 3D surface deformation results, among which the UTA team only obtained results for $\beta=90^{\circ}$ as the new 3D implementation of CFRAC_UT only handles vertical fractures (McClure et al. 2015). The results for the three dipping angles and two sampling lines (along the $\mathrm{x}$-axis and $\mathrm{z}$-axis) are shown in Fig. 11-6 to Fig. 11-8. As the plane-strain 2D model is equivalent to a 3D model with infinite out-of-plane depth (i.e. $b$ approaches infinity), the $3 \mathrm{D}$ surface deformation at $z=0$ is expected to be smaller than the $2 \mathrm{D}$ solution. The $3 \mathrm{D}$ solutions overall are very similar to each other, although the difference is somewhat more pronounced than that for the $2 \mathrm{D}$ results. For $\beta=0^{\circ}$ and $45^{\circ}$, the OU solutions still overpredict the surface deformation, consistent with the trend in $2 \mathrm{D}$. For the case with $\beta=0^{\circ}$, the OU deformation is even greater than the $2 \mathrm{D}$ reference solution, a clear evidence of some inaccuracy. However, the OU 3D deformation is smaller than their 2D deformation, passing a self-consistency check. For the case with $\beta=90^{\circ}$, the results of all four teams are very similar, showing smaller surface deformation than the 2D reference solution, as expected. 


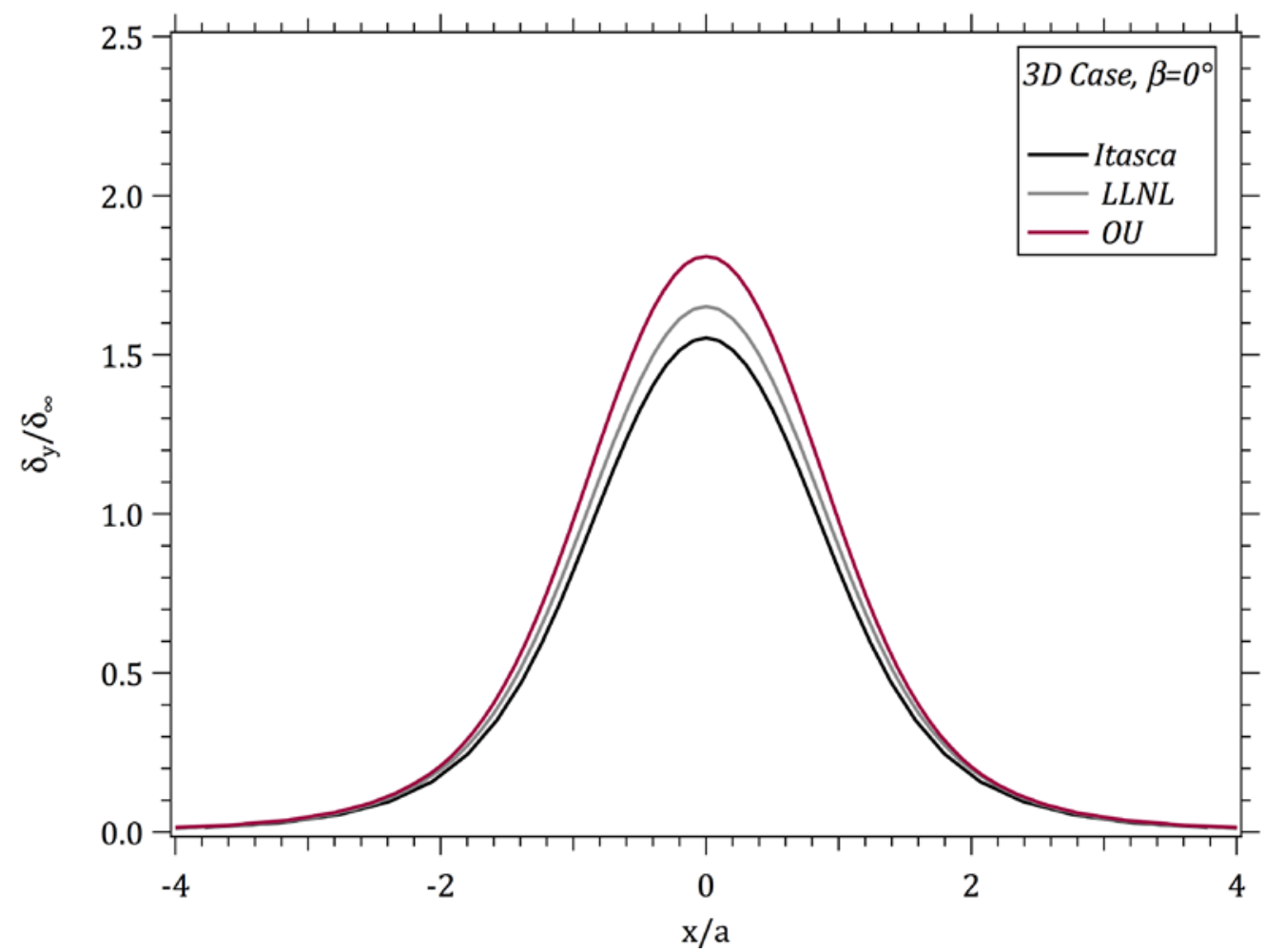

Figure 11-6. Vertical surface displacement $\delta_{y}$ normalized by $\delta_{\infty}$ from $\mathrm{x}=-4 \mathrm{a}$ to $4 \mathrm{a}$ for $\beta=0^{\circ}$ for the 3D

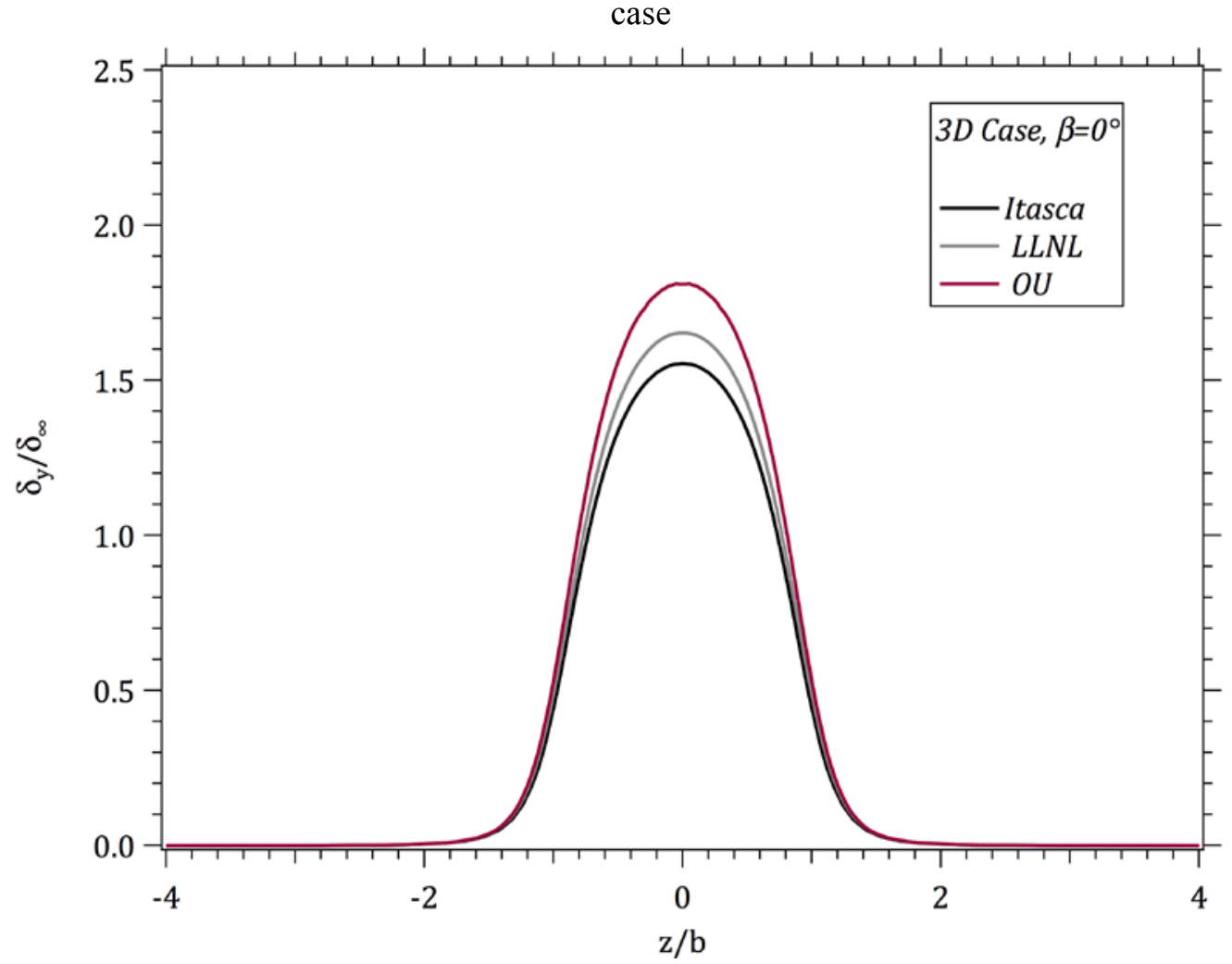

Figure 11-7. Vertical surface displacement $\delta_{y}$ normalized by $\delta_{\infty}$ from $\mathrm{z}=-4 \mathrm{~b}$ to $4 \mathrm{~b}$ for $\beta=0^{\circ}$ for the 3D case 


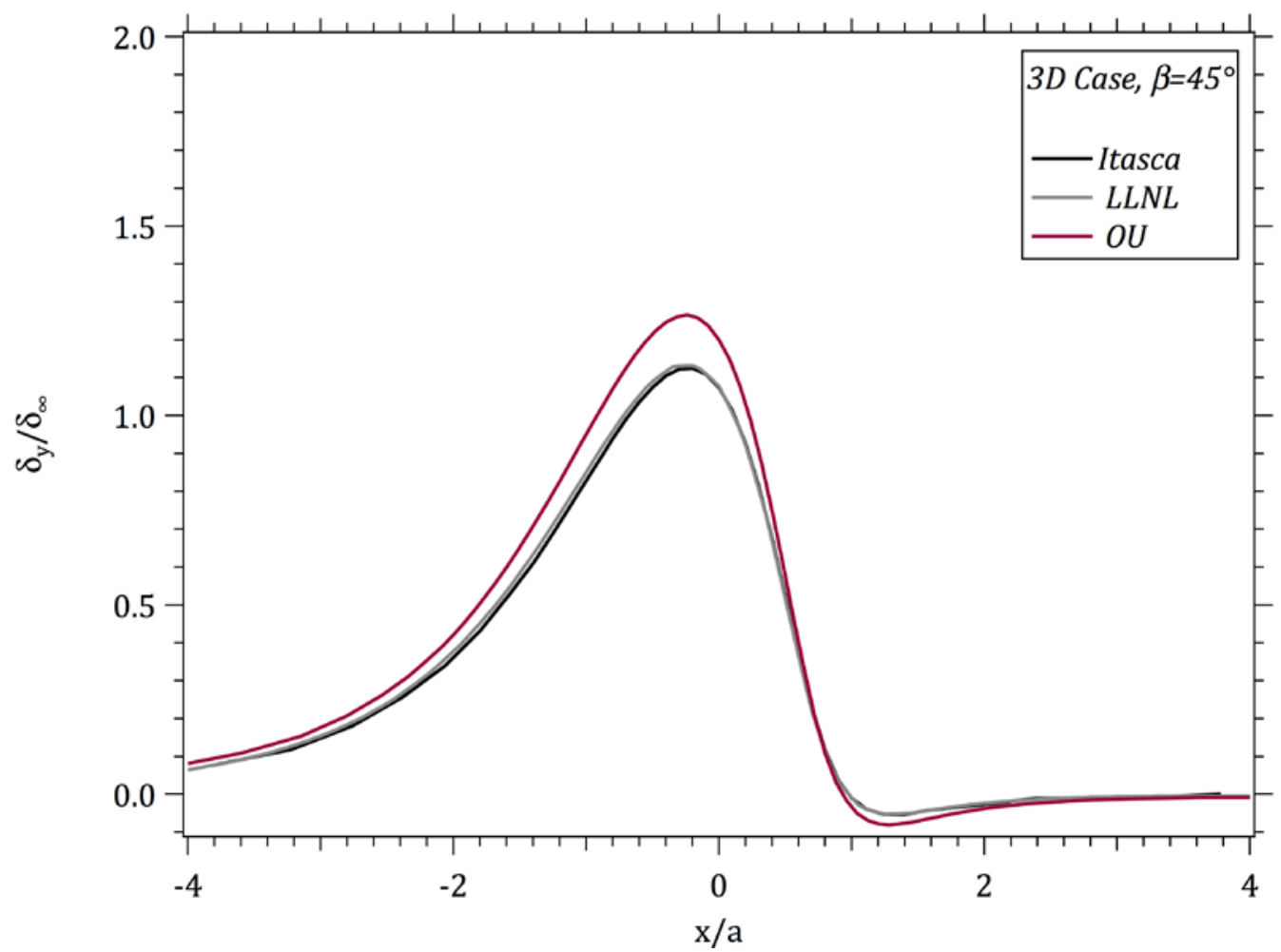

Figure 11-8. Vertical surface displacement $\delta_{y}$ normalized by $\delta_{\infty}$ from $\mathrm{x}=-4 \mathrm{a}$ to $4 \mathrm{a}$ for $\beta=45^{\circ}$ for the 3D case

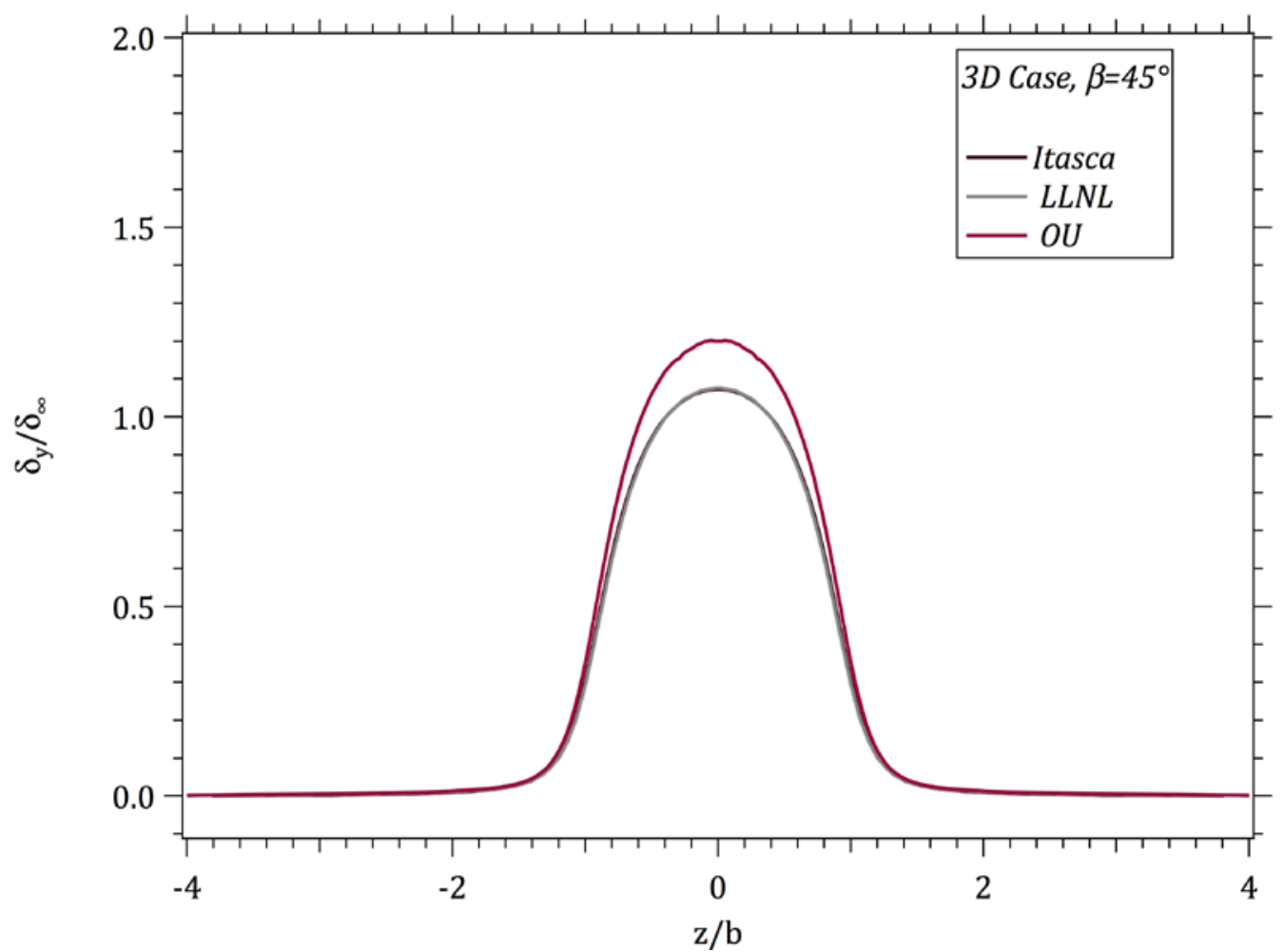

Figure 11-9. Vertical surface displacement $\delta_{y}$ normalized by $\delta_{\infty}$ from z $=-4 \mathrm{~b}$ to $4 \mathrm{~b}$ for $\beta=45^{\circ}$ for the 3D case 


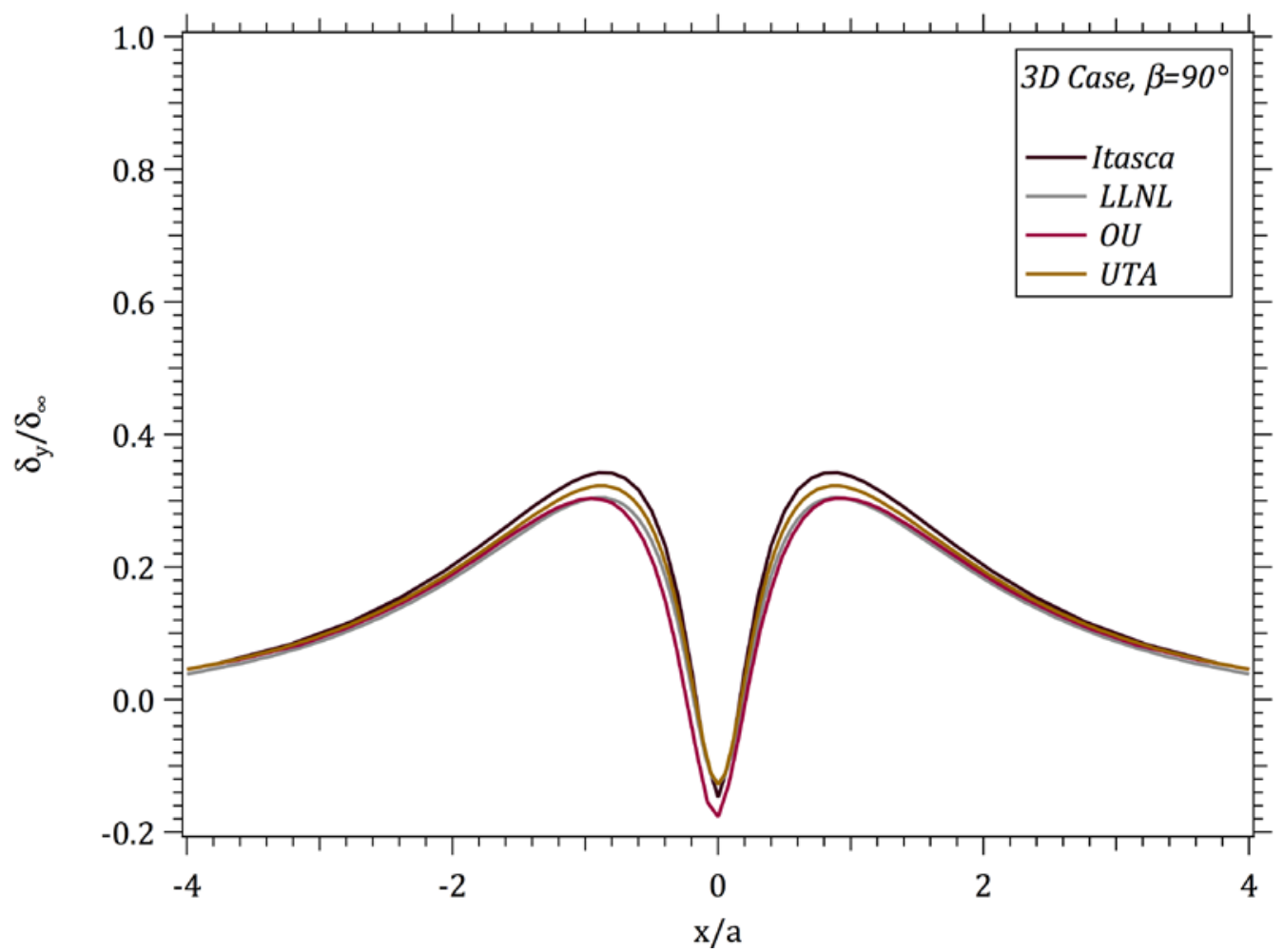

Figure 11-10. Vertical surface displacement $\delta_{y}$ normalized by $\delta_{\infty}$ from $\mathrm{x}=-4 \mathrm{a}$ to $4 \mathrm{a}$ for $\beta=90^{\circ}$ for the 3D case

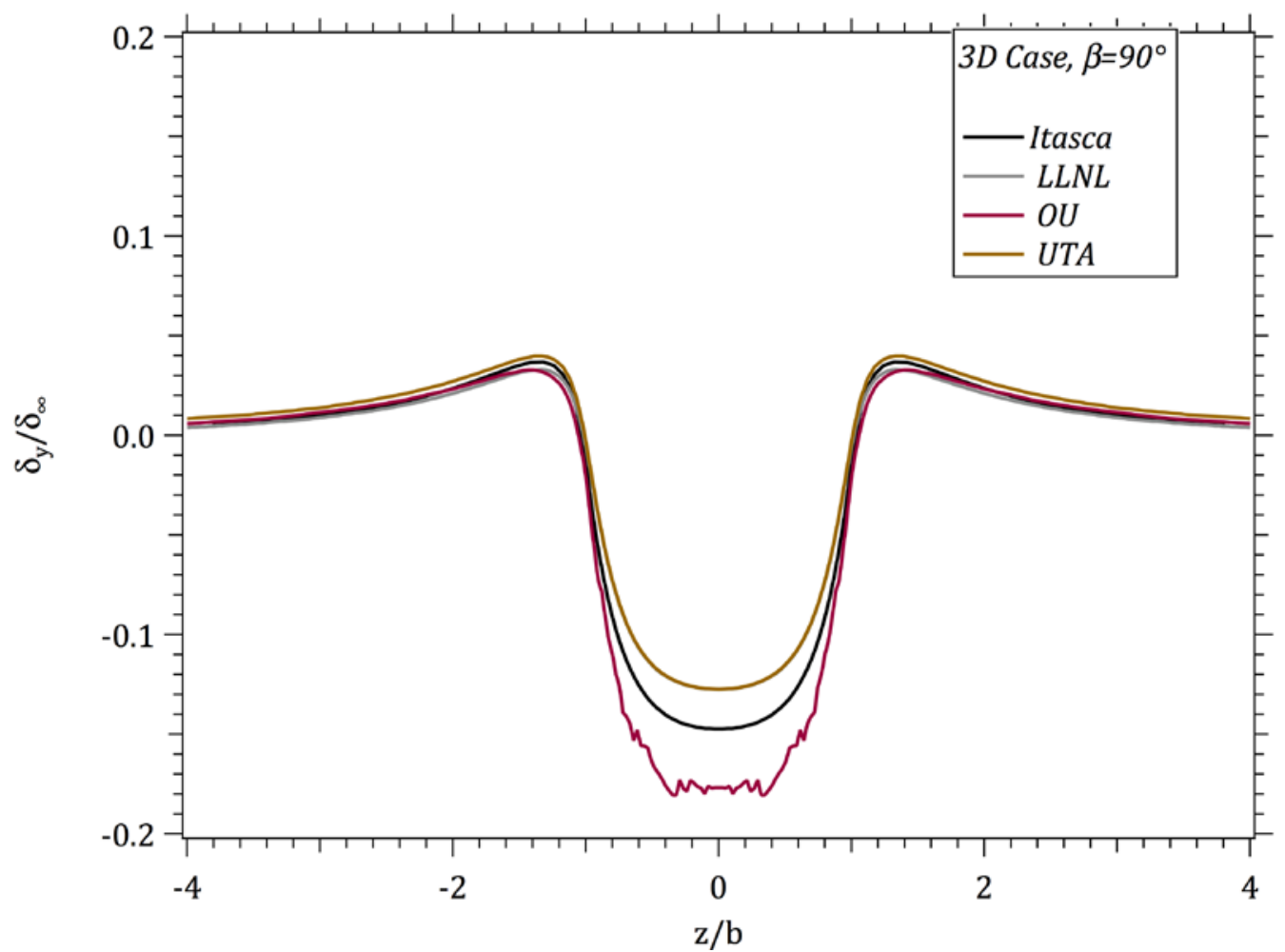

Figure 11-11. Vertical surface displacement $\delta_{y}$ normalized by $\delta_{\infty}$ from z $=-4 \mathrm{~b}$ to $4 \mathrm{~b}$ for $\beta=90^{\circ}$ for the 3D case 


\subsection{Comparison of the stress intensity factor results}

Although the problem specifications called for SIF results, submissions of such results turned out to be rare. Only the LLNL team, the designer of the problem, submitted a complete set of SIF results. Their 2D results are very similar to those in (Pollard and Holzhausen 1979) but we do not show those here since there is no result from other teams to compare with. The UTA team submitted 3D SIF solutions for the case of $\beta=90^{\circ}$ along the long edge near the surface as this is the only geometry allowed by CFRAC_UT. The comparison of the 3D SIF results between the LLNL and UTA teams is show in Fig. 11-12. The results are almost identical to each other, although LLNL's GEOS and UT's CFRAC_UT are based on very different numerical methods: Finite Element Method and Boundary Element Method, respectively.

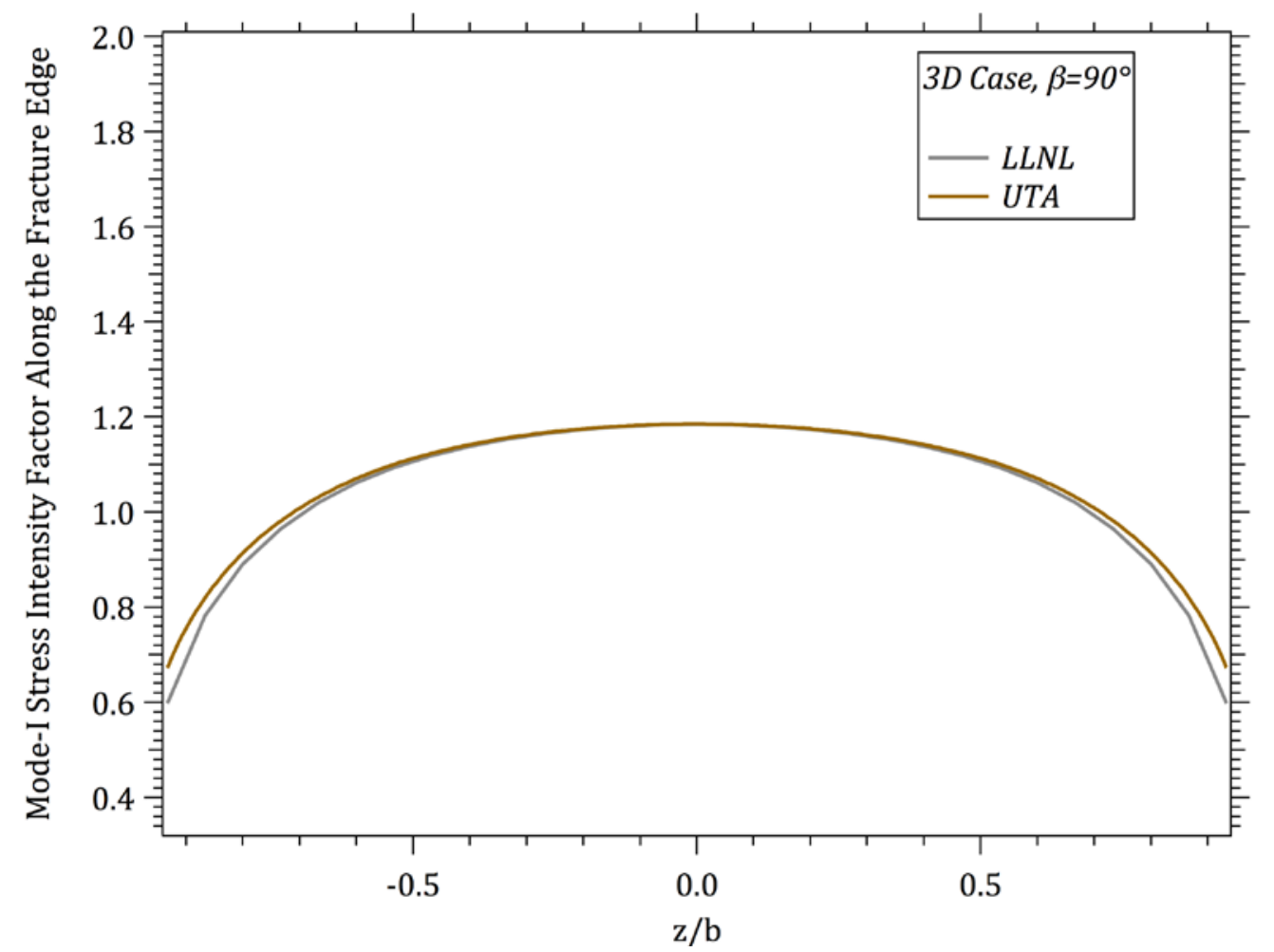

Figure 11-12. The mode-I stress intensity factor at the two fracture tips for $\beta=90^{\circ}$ for the $3 \mathrm{D}$ case

\subsection{Discussion of Problem 7 Results}

Overall, the surface deformation predictions made by all the participating teams are very similar. This is especially encouraging considering the variety of numerical methods (FEM, FEM, BEM, and bonded particle type method) used. The analysis of the results revealed the importance of using sufficiently large domain sizes to approximate the infinite domain and using appropriate mesh resolutions. Unfortunately, most teams did not respond to the request for stress intensity factor predictions and therefore a comprehensive comparison was not possible. This demonstrates deficiencies of current codes in handling basic fracture mechanics principles, which are essential for unconventional reservoir stimulation where fracture mechanics plays a central role. These aspects are usually not considered in traditional geothermal reservoir modeling. 


\subsection{Conclusions}

Numerical simulation provides scientists and engineers with analytical tools for understanding complex physical processes and the capabilities of current multiprocessor workstation computers allow the consideration of coupled processes. For EGS, hydrologic, thermal, geomechanical and geochemical processes all contribute to realizing the energy potentials from geothermal resources. The inherent heterogeneity of the earth's crust contributes greatly to the uncertainty in modeling EGS via numerical simulation, but the modeling of more idealized systems provides opportunities for an in-depth understanding of the impacts of design and field operational choices.

In 1980 the geothermal community dedicated the annual Geothermal Reservoir Engineering workshop to defining an appropriate role for numerical simulation in terms of investment decisions related to geothermal performance predictions and to assessing the state of development of geothermal reservoir numerical simulators. The technical foundation for achieving the workshop objectives was a code comparison study, which involved six geothermal problems. Whereas the suite of problems considered a variety of geometric configurations and petrophysical property distributions, the principal processes of concern were single-phase flow, two-phase flow, single-phase to two-phase flash, and heat transfer (i.e., TH processes). This study is similar to the 1980 code comparison study in that the objectives are nearly unchanged: an assessment of computer codes for predicting the power potential and longevity of geothermal reservoirs. Today's numerical simulators for EGS, however, have evolved from those of the 1980 s, particularly with respect to modeling coupled processes. In alignment with this transition in simulation capabilities, all of the benchmark problems in this study included coupled process elements of either HM, THM, or THC. This study has demonstrated that while the U.S. EGS simulation community has a diverse set of computational tools with respect to conceptual approaches, they are able to simulate coupled subsurface processes with comparable results, as evidenced by the benchmark problem solutions.

The evolution of numerical simulators over the last thirty five years has been impressive, but work remains to be done. Uncertainty in simulation results as measured by the ISO-13528 standard tend to increase with the number of coupled processes in the problem and the modeling of strongly coupled THMC processes remains challenging. The collaborative nature of this study has formed the foundation for the EGS simulation community to collectively address field-scale systems, where coupled process modeling will be essential for understanding the system and experimental observations. Confidence in numerical simulation grows from agreement among field experts, especially when diverse perspectives are represented. This study yielded convergence in understanding over the course of each problem via open dialogue and discussions among the participants. 



\subsection{References}

Anderson, BJ, JW Wilder, M Kurihara, MD White, GJ Moridis, SJ Wilson, M Pooladi-Darvish, Y Masuda, T Collett, and R Hunter. 2008. Analysis of modular dynamic formation test results from the 'Mount Elbert' stratigraphic test well, Milne Point, Alaska. In Proceedings of 6th International Conference on Gas Hydrates, Vancouver, British Columbia, Canada, ICGH,

Aradóttir, ESP, EL Sonnenthal, G Björnsson, and H Jónsson. 2012. "Multidimensional reactive transport modeling of $\mathrm{CO} 2$ mineral sequestration in basalts at the Hellisheidi geothermal field, Iceland," International Journal of Greenhouse Gas Control, 9(0):24-40. doi:http://dx.doi.org/10.1016/j.ijggc.2012.02.006.

Asahina, D, JE Houseworth, JT Birkholzer, J Rutqvist, and JE Bolander. 2014. "Hydro-mechanical model for wetting/drying and fracture development in geomaterials," Computers \& Geosciences, 65:13-23. doi:DOI 10.1016/j.cageo.2013.12.009.

Bahrami, D, G Danko, P Fu, B Guo, R Podgorney, M White, and Y Xia. 2015. Poroelastic and SelfPropped Single Fracture THM Models for EGS Studies. In Proceedings Stanford, CA,

Bai, M and D Elsworth. 2000. Coupled Processes in Subsurface Deformation, Flow, and Transport. American Society of Civil Engineers.

Bandis, SC, AC Lumsden, and NR Barton. 1983. "Fundamentals of Rock Joint Deformation," International Journal of Rock Mechanics and Mining Sciences, 20(6):249-268. doi:Doi 10.1016/01489062(83)90595-8.

Barton, N. 1976. "The shear strength of rock and rock joints," International Journal of Rock Mechanics and Mining Sciences \& Geomechanics Abstracts, 13(9):255-279. doi:http://dx.doi.org/10.1016/01489062(76)90003-6.

Barton, N. 2013. "Shear strength criteria for rock, rock joints, rockfill and rock masses: Problems and some solutions," Journal of Rock Mechanics and Geotechnical Engineering, 5(4):249-261. doi:http://dx.doi.org/10.1016/j.jrmge.2013.05.008.

Barton, N, S Bandis, and K Bakhtar. 1985. "Strength, Deformation and Conductivity Coupling of Rock Joints," International Journal of Rock Mechanics and Mining Sciences, 22(3):121-140. doi:Doi 10.1016/0148-9062(85)93227-9.

Bradford, J, J Mclennan, J Moore, D Glasby, D Waters, R Kruwell, A Bailey, W Rickard, K Bloomfield, and D King. 2013. Recent Developments at the Raft River Geothermal Field. In Proceedings of ThirtyEighth Workshop on Geothermal Reservoir Engineering, Stanford University, Stanford, CA,

Bradford, J, J Moore, M Ohren, J Mclenna, WL Osborn, E Majer, G Nash, RK Podgorney, B Freifeld, R Nye, W Rickard, D Waters, and D Glaspey. 2015. Recent Thermal and Hydraulic Stimulation Results at Raft River, ID EGS Site. In Proceedings of Fourtieth Workshop on Geothermal Reservoir Engineering, Stanford, CA, USA, Stanford University,

Brebbia, CA and J Dominguez. 1977. "Boundary Element Methods for Potential Problems," Applied Mathematical Modelling, 1(7):372-378. doi:Doi 10.1016/0307-904x(77)90046-4. 
Chabora, E, E Zemach, P Spielman, P Drakos, S Hickman, S Lutz, K Boyle, A Falconer, A RobertsonTait, NC Davatzes, P Rose, M E., and S Jarpe. 2012a. Hydraulic Stimulation of Well 27-15, Desert Peak Geothermal Field, Nevada USA. In In Proceedings of 37th Workshop on Geothermal Reservoir Engineering, Stanford University, Stanford, CA, Stanford, CA.

Chabora, E, E Zemach, P Spielman, P Drakos, S Hickman, S Lutz, K Boyle, A Falconer, A RobertsonTait, NC Davatzes, P Rose, E Majer, and S Jarpe. 2012b. Hydraulic Stimulation of Well 27-15, Desert Peak Geothermal Field, Nevada USA. In Proceedings of 37th Workshop on Geothermal Reservoir Engineering, Stanford, California, USA, Stanford University,

Cheng, CL, MJ Gragg, E Perfect, MD White, PJ Lemiszki, and LD Mckay. 2013. "Sensitivity of injection costs to input petrophysical parameters in numerical geologic carbon sequestration models," International Journal of Greenhouse Gas Control, 18:277-284. doi:DOI 10.1016/j.ijggc.2013.07.018.

Class, H, A Ebigbo, R Helmig, HK Dahle, JM Nordbotten, MA Celia, P Audigane, M Darcis, J EnnisKing, YQ Fan, B Flemisch, SE Gasda, M Jin, S Krug, D Labregere, AN Beni, RJ Pawar, A Sbai, SG Thomas, L Trenty, and LL Wei. 2009. "A benchmark study on problems related to CO2 storage in geologic formations," Computational Geosciences, 13(4):409-434. doi:10.1007/s10596-009-9146-x.

Coats, KH. 1977. Geothermal Reservoir Modelling. In Proceedings of SPE Annual Fall Technical Conference and Exhibition, Denver, CO, Society of Petroleum Engineers, SPE.

Crouch, SL. 1976. "Solution of Plane Elasticity Problems by Displacement Discontinuity Method .1. Infinite Body Solution," International Journal for Numerical Methods in Engineering, 10(2):301-343. doi:DOI 10.1002/nme.1620100206.

Crouch, SL and AM Starfield. 1983. Boundary Element Methods in Solid Mechanics: With Applications in Rock Mechanics and Geological Engineering. Allen \& Unwin.

Danko, G. 2006. "Functional or operator representation of numerical heat and mass transport models," Journal of Heat Transfer-Transactions of the Asme, 128(2):162-175. doi:Doi 10.1115/1.2136919.

Danko, G. 2008. MULTIFLUX V5.0 Software Documentation Qualification Documents, Software Tracking Number: 1002-5.0-00, Laurence Berkeley National Laboratory, Berkeley, CA.

Danko, G and D Bahrami. 2012. "A new T-H-M-C model development for discrete-fracture EGS studies," Geothermal Resources Council Transactions, 36:383-391.

Danko, G and D Bahrami. 2013a. Dynamic Model of Discrete Fracture Opening Under Fluid Injection. In Proceedings of Thirty-Eighth Workshop on Geothermal Reservoir Engineering, Stanford University, Stanford, CA,

Danko, G and D Bahrami. 2013b. "EGS Model Adaptation and Forecasting Studies with MULTIFLUX," Geothermal Resources Council Transactions, 37:141-147.

Danko, G, D Bahrami, and Z L. 2012. Coupled MULTIFLUX-TOUGH2-TOUGHREACT THMC model for EGS studies. In Proceedings of TOUGH Symposium 2012, Lawrence Berkeley National Laboratory, Berkeley, California, 
Deb, D. 2006. Finite Element Method: Concepts and Applications in Geomechanics. Prentice-Hall of India.

Dempsey, D, S Kelkar, K Lewis, S Hickman, N Davatzes, D Moos, and E Zemach. Modeling Shear Stimulation of the Desert Peak EGS Well 27-15 Using a Coupled Thermal-Hydrological-Mechanical Simulator. In Proceedings American Rock Mechanics Association, ARMA.

Dempsey, D, S Kelkar, K Lewis, S Hickman, N Davatzes, D Moos, and E Zemach. 2013. Modeling Shear Stimulation of the Desert Peak EGS Well 27-15 Using a Coupled Thermal-Hydrological-Mechanical Simulator. In Proceedings of 47th U.S. Rock Mechanics/Geomechanics Symposium, San Francisco, CA, American Rock Mechanics Association, ARMA.

Dobroskok, A and A Ghassemi. 2005. "Deformation and Stability of a Discontinuity in a Geothermal System," GRC Transactions, 29.

Dobson, PF, TJ Kneafsey, EL Sonnenthal, N Spycher, and JA Apps. 2003. "Experimental and numerical simulation of dissolution and precipitation: implications for fracture sealing at Yucca Mountain, Nevada," Journal of Contaminant Hydrology, 62-3:459-476. doi:10.1016/S0169-7722(02)00155-9.

Dobson, PF, S Salah, N Spycher, and EL Sonnenthal. 2004. "Simulation of water-rock interaction in the Yellowstone geothermal system using TOUGHREACT," Geothermics, 33(4):493-502. doi:http://dx.doi.org/10.1016/j.geothermics.2003.10.002.

Donaldson, IG and ML Sorey. 1979. The Best Uses of Numerical Simulators. In Proceedings of Fifth Workshop on Geothermal Reservoir Engineering, Stanford University, Stanford, California,

Drucker, DC and W Prager. 1952. "Soil Mechanics and Plastic Analysis or Limit Design," Quarterly of Applied Mathematics, 10(2):157-165.

Elsworth, D and M Bai. 1992. "Flow-Deformation Response of Dual-Porosity Media," Journal of Geotechnical Engineering-Asce, 118(1):107-124. doi:Doi 10.1061/(Asce)0733-9410(1992)118:1(107).

Eslinger, PW, LA Doremus, DW Engel, TB Miley, MT Murphy, WE Nichols, MD White, DW Langford, and SJ Ouderkirk. 1993. Preliminary total-system analysis of a potential high-level nuclear waste repository at Yucca Mountain, PNL--8444; Other: ON: DE93007438; TRN: 93:006338 United States10.2172/138891Other: ON: DE93007438; TRN: 93:006338Mon Feb 04 17:28:14 EST 2008INIS; OSTI as DE93007438PNNL; SCA: 052002; 580000; PA: EDB-93:045223; NTS-93:011869; ERA18:012121; SN: 93000934572English.

Fairley, JP, SE Ingebritsen, and RK Podgorney. 2010. "Challenges for Numerical Modeling of Enhanced Geothermal Systems," Ground Water, 48(4):482-483. doi:10.1111/j.1745-6584.2010.00716.x.

Finsterle, S, EL Sonnenthal, and N Spycher. 2014. "Advances in subsurface modeling using the TOUGH suite of simulators," Computers \& Geosciences, 65(0):2-12.

doi:http://dx.doi.org/10.1016/j.cageo.2013.06.009.

Fu, P, Y Hao, and CR Carrigan. 2013. A geomechanical mechanism that counteracts flow channeling induced by reservoir thermal drawdown. In Proceedings of Thirty-Eighth Workshop on Geothermal Reservoir Engineering, Stanford University, Stanford, CA, 
Garipov, TT, M Karimi-Fard, and HA Tchelepi. 2016. "Discrete fracture model for coupled flow and geomechanics," Computational Geosciences:1-12. doi:10.1007/s10596-015-9554-z.

Garipov, TT, KA Lovonyan, M Karimi-Fard, and HA Tchelepi. 2012. Coupled Geomechanics and Flow in Fractured Media. In Proceedings of ECMOR XIII - 13th European Conference on the Mathematics of Oil Recovery,

Gaston, D, L Guo, G Hansen, H Huang, R Johnson, H Park, R Podgorney, M Tonks, and R Williamson. 2012a. "Parallel Algorithms and Software for Nuclear, Energy, and Environmental Applications Part I: Multiphysics Algorithms," Communications in Computational Physics (Print), 12(3):807-833.

Gaston, D, L Guo, G Hansen, H Huang, R Johnson, H Park, R Podgorney, M Tonks, and R Williamson. 2012b. "Parallel Algorithms and Software for Nuclear, Energy, and Environmental Applications Part II: Multiphysics Software," Communications in Computational Physics (Print), 12(3):834-865.

Gaston, D, G Hansen, S Kadioglu, DA Knoll, C Newman, H Park, C Permann, and W Taitano. 2009. "Parallel multiphysics algorithms and software for computational nuclear engineering," Scidac 2009: Scientific Discovery through Advanced Computing, 180. doi:Doi 10.1088/1742-6596/180/1/012012.

Gaston, DR, CJ Permann, JW Peterson, AE Slaughter, D Andrš, Y Wang, MP Short, DM Perez, MR Tonks, J Ortensi, L Zou, and RC Martineau. 2014a. "Physics-based multiscale coupling for full core nuclear reactor simulation," Annals of Nuclear Energy, (0). doi:http://dx.doi.org/10.1016/j.anucene.2014.09.060.

Gaston, DR, JW Peterson, CJ Permann, D Andrs, AE Slaughter, and JM Miller. 2014b. "Continuous Integration for Concurrent Computational Framework and Application Development," 2014, 2(1). doi: $10.5334 /$ jors.as e10.

Ghassemi, A and Q Zhang. 2006. "Porothermoelastic analysis of the response of a stationary crack using the displacement discontinuity method," Journal of Engineering Mechanics-Asce, 132(1):26-33. doi:Doi 10.1061/(Asce)0733-9399(2006)132:1(26).

Gorton, I, C Sivaramakrishnan, G Black, S White, S Purohit, C Lansing, M Madison, K Schuchardt, and Y Lou. 2012. "Velo: A Knowledge-Management Framework for Modeling and Simulation," Computing in Science \& Engineering, 14(2):12-23.

Hackett, P. 1959. "An elastic analysis of rock movements caused by mining," Transactions of Int. Min. Eng., 118(7):421-433.

Hao, Y, P Fu, and CR Carrigan. 2013. Application of a dual-continuum model for simulation of fluid flow and heat transfer in fractured geothermal reservoirs. In Proceedings of 38th Stanford Geothermal Workshop, Stanford University, Stanford, CA, Stanford, CA.

Harris, RA, M Barall, R Archuleta, E Dunham, B Aagaard, JP Ampuero, H Bhat, V Cruz-Atienza, L Dalguer, P Dawson, S Day, B Duan, G Ely, Y Kaneko, Y Kase, N Lapusta, Y Liu, S Ma, D Oglesby, K Olsen, A Pitarka, S Song, and E Templeton. 2009. "The SCEC/USGS Dynamic Earthquake Rupture Code Verification Exercise," Seismological Research Letters, 80(1):119-126. doi:10.1785/gssrl.80.1.119.

Hoek, E and ET Brown. 1980. Underground Excavations in Rock. Institution of Mining and Metallurgy. 
Huang, H, M Plummer, and RK Podgorney. 2013. Simulated Evolution of Fractures and Fracture Networks Subject to Thermal Cooling and Fluid Pressure Changes: A Coupled Network Flow and Discrete Element Model. In Proceedings of Thirty-Eighth Workshop on Geothermal Reservoir Engineering, Stanford University, Stanford, CA.,

Hudson, JA and L Jing. 2013. "Demonstration of coupled models and their validation against experiment: The current phase DECOVALEX 2015," Rock Characterisation, Modelling and Engineering Design Methods:391-396.

Iranshahr, A, DV Voskov, and HA Tchelepi. 2010. "Tie-Simplex Parameterization for EOS-Based Thermal Compositional Simulation," Spe Journal, 15(2):545-556.

Iso 13528. 2005. Statistical methods for use in proficiency testing by interlaboratory comparisons, International Organization for Standardization, Geneva, Switzerland.

Itasca Consulting Group, I. 2011. FLAC - Fast Lagrangian Analysis of Continua, Version. 7.0. Itasca, Minneapolis, MN.

Itasca Consulting Group, I. 2013. 3DEC - Three-Dimensional Distinct Element Code, Version 5.0. Itasca, Minneapolis, MN

Itasca Consulting Group, I. 2014. FLAC3D - Fast Lagrangian Analysis of Continua in ThreeDimensions, Version. 5.0. Itasca, Minneapolis, MN.

Jaeger, JC, NGW Cook, and R Zimmerman. 2007. Fundamentals of Rock Mechanics. Wiley-Blackwell.

Jeanne, P, J Rutqvist, PF Dobson, M Walters, C Hartline, and J Garcia. 2014. "The impacts of mechanical stress transfers caused by hydromechanical and thermal processes on fault stability during hydraulic stimulation in a deep geothermal reservoir," International Journal of Rock Mechanics and Mining Sciences, 72:149-163. doi:DOI 10.1016/j.ijrmms.2014.09.005.

Jung, R. 2013. " EGS — Goodbye or Back to the Future 95." In Effective and Sustainable Hydraulic Fracturing, ed. D.R. Jeffrey. InTech.

Kachanov, LM. 1958. "Time of the rupture process under creep conditions," Isv. Akad. Nauk. SSR. Otd Tekh. Nauk, 8:26-31.

Karimi-Fard, M, LJ Durlofsky, and K Aziz. 2004. "An efficient discrete-fracture model applicable for general-purpose reservoir simulators," Spe Journal, 9(2):227-236. doi:Doi 10.2118/88812-Pa.

Kelkar, S, K Lewis, S Karra, G Zyvoloski, S Rapaka, H Viswanathan, PK Mishra, S Chu, D Coblentz, and R Pawar. 2014. "A simulator for modeling coupled thermo-hydro-mechanical processes in subsurface geological media," International Journal of Rock Mechanics and Mining Sciences, 70(0):569-580. doi:http://dx.doi.org/10.1016/j.ijrmms.2014.06.011.

Kelkar, S, G Srinivasan, BA Robinson, R Roback, H Viswanathan, K Rehfeldt, and P Tucci. 2013. "Breakthrough of contaminant plumes in saturated volcanic rock: implications from the Yucca Mountain site," Geofluids, 13(3):273-282. doi:DOI 10.1111/gfl.12035. 
Kim, J, E Sonnenthal, and J Rutqvist. 2015. "A sequential implicit algorithm of chemo-thermo-poromechanics for fractured geothermal reservoirs," Computers \& Geosciences, 76:59-71. doi:DOI 10.1016/j.cageo.2014.11.009.

Kim, J, EL Sonnenthal, and J Rutqvist. 2012. "Formulation and sequential numerical algorithms of coupled fluid/heat flow and geomechanics for multiple porosity materials," International Journal for Numerical Methods in Engineering, 92(5):425-456. doi:DOI 10.1002/nme.4340.

Kim, J, HA Tchelepi, and R Juanes. 2011. "Stability and convergence of sequential methods for coupled flow and geomechanics: Fixed-stress and fixed-strain splits," Computer Methods in Applied Mechanics and Engineering, 200(13-16):1591-1606. doi:DOI 10.1016/j.cma.2010.12.022.

Kohl, T and T Megel. 2007. "Predictive modeling of reservoir response to hydraulic stimulations at the European EGS site Soultz-sous-Forets," International Journal of Rock Mechanics and Mining Sciences, 44(8):1118-1131. doi:DOI 10.1016/j.ijrmms.2007.07.022.

Kourounis, D, D Voskov, and K Aziz. 2010. Adjoint Methods for Multicomponent Flow Simulation. In Proceedings of 12th European Conference on the Mathematics of Oil Recovery, Oxford, United Kingdom,

Lee, SH and A Ghassemi. 2011. Three-Dimensional Thermo-Poro-Mechanical Modeling Of Reservoir Stimulation And Induced Microseismicity In Geothermal Reservoir In Proceedings of Thirty-Sixth Workshop on Geothermal Reservoir Engineering Stanford University, Stanford, California,

Lenhard, RJ, M Oostrom, and MD White. 1995. "Modeling Fluid-Flow and Transport in Variably Saturated Porous-Media with the Stomp Simulator .2. Verification and Validation Exercises," Advances in Water Resources, 18(6):365-373. doi:Doi 10.1016/0309-1708(95)00019-F.

Mandl, G. 2000. Faulting in Brittle Rocks: An Introduction to the Mechanics of Tectonic Faults. Springer.

Mcclure, M. 2014. The Potential Effect of Network Complexity on Recovery of Injected Fluid Following Hydraulic Fracturing. In Proceedings Society of Petroleum Engineers, SPE.

Mcclure, M, M Babazadeh, S Shiozawa, and J Huang. 2015. Fully Coupled Hydromechanical Simulation of Hydraulic Fracturing in Three-Dimensional Discrete Fracture Networks. In Proceedings Society of Petroleum Engineers, SPE.

Mcclure, M and RN Horne. 2013. Discrete Fracture Network Modeling of Hydraulic Stimulation: Coupling Flow and Geomechanics. 1 ed., SpringerBriefs in Earth Sciences, Springer International Publishing.

Mcclure, MW. 2012. "Modeling and characterization of hydraulic stimulation and induced seismicity in geothermal and shale gas reservoirs," Ph.D., Stanford University, Stanford University.

Mcclure, MW and RN Horne. 2014. "An investigation of stimulation mechanisms in Enhanced Geothermal Systems," International Journal of Rock Mechanics and Mining Sciences, 72(0):242-260. doi:http://dx.doi.org/10.1016/j.ijrmms.2014.07.011.

Mcgrail, BP, DH Bacon, JP Icenhower, FM Mann, RJ Puigh, HT Schaef, and SV Mattigod. 2001. "Nearfield performance assessment for a low-activity waste glass disposal system: laboratory testing to 
modeling results," Journal of Nuclear Materials, 298(1-2):95-111. doi:Doi 10.1016/S0022-

3115(01)00576-1.

Mcgrail, BP, JE Cabe, CL Davidson, FS Knudsen, DH Bacon, MD Bearden, MA Chamness, JA Horner, SP Reidel, HT Schaef, FA Spane, and PD Thorne. 2013. Techno-economic Performance Evaluation of Compressed Air Energy Storage in the Pacific Northwest Pacific Northwest National Laboratory, Richland, WA

Mercer, JW and GF Pinder. 1973. "Galerkin Finite-Element Simulation of a Geothermal Reservoir," Geothermics, 2(3-4).

Min, K-B, J Rutqvist, and D Elsworth. 2009. "Chemically and mechanically mediated influences on the transport and mechanical characteristics of rock fractures," International Journal of Rock Mechanics and Mining Sciences, 46(1):80-89. doi:http://dx.doi.org/10.1016/j.ijrmms.2008.04.002.

Moes, N, J Dolbow, and T Belytschko. 1999. "A finite element method for crack growth without remeshing," International Journal for Numerical Methods in Engineering, 46(1):131-150. doi:Doi 10.1002/(Sici)1097-0207(19990910)46:1<131::Aid-Nme726>3.3.Co;2-A.

Mohammadi, S. 2008. Extended Finite Element Method: for Fracture Analysis of Structures. Wiley.

Mohr, O. 1914. Abhandlungen aus dem Gebiete der technischen Mechanik, von... Otto Mohr,... 2te... Auflage. W. Ernst und Sohn.

Molins, S, J Greskowiak, C Wanner, and KU Mayer. 2015. "A benchmark for microbially mediated chromium reduction under denitrifying conditions in a biostimulation column experiment," Computational Geosciences, 19(3):479-496. doi:10.1007/s10596-014-9432-0.

Mukhopadhyaya, S, Z Houb, L Gosink, D Bacon, C Doughty, JJ Li, L Wei, S Gasda, G Bacci, R Govindan, J-Q Shi, H Yamamoto, R Ramanathan, JP Nicot, SA Hosseini, JT Birkholzer, and A Bonneville. 2013. "The Sim-SEQ Initiative," Energy Procedia, 37:3867-3874. doi:doi: 10.1016/j.egypro.2013.06.284.

Murphy, HD, JW Tester, CO Grigsby, and RM Potter. 1981. "Energy extraction from fractured geothermal reservoirs in low-permeability crystalline rock," Journal of Geophysical Research: Solid Earth, 86(B8):7145-7158. doi:10.1029/JB086iB08p07145.

Mutlu, O and DD Pollard. 2008. "On the patterns of wing cracks along an outcrop scale flaw: A numerical modeling approach using complementarity," Journal of Geophysical Research: Solid Earth, 113(B6):n/a-n/a. doi:10.1029/2007JB005284.

Nathenson, M. 1999. "The dependence of permeability on effective stress from flow tests at hot dry rock reservoirs at Rosemanowes (Cornwall) and Fenton Hill (New Mexico)," Geothermics, 28(3):315-340. doi:http://dx.doi.org/10.1016/S0375-6505(99)00011-5.

Nitao, JJ. 2000. Reference Manual for the NUFT Flow and Transport Code Version 3.0, Lawrence Livermore National Laboratory, Livermore, CA.

Okada, Y. 1985. "Surface Deformation Due to Shear and Tensile Faults in a Half-Space," Bulletin of the Seismological Society of America, 75(4):1135-1154. 
Okada, Y. 1992. "Internal Deformation Due to Shear and Tensile Faults in a Half-Space," Bulletin of the Seismological Society of America, 82(2):1018-1040.

Oostrom, M, PD Thorne, MD White, MJ Truex, and TW Wietsma. 2003. "Numerical modeling to assess DNAPL movement and removal at the scenic site operable unit near Baton Rouge, Louisiana: A case study," Soil \& Sediment Contamination, 12(6):901-926. doi:Doi 10.1080/10588330390254946.

Pan, PZ, J Rutqvist, XT Feng, F Yan, and Q Jiang. 2014. "A Discontinuous Cellular Automaton Method for Modeling Rock Fracture Propagation and Coalescence Under Fluid Pressurization Without Remeshing," Rock Mechanics and Rock Engineering, 47(6):2183-2198. doi:DOI 10.1007/s00603-0130522-4.

Pande, GN, G Beer, and JR Williams. 1990. Numerical methods in rock mechanics. Wiley.

Pandey, SN, A Chaudhuri, S Kelkar, VR Sandeep, and H Rajaram. 2014. "Investigation of permeability alteration of fractured limestone reservoir due to geothermal heat extraction using three-dimensional thermo-hydro-chemical (THC) model," Geothermics, 51(0):46-62.

doi:http://dx.doi.org/10.1016/j.geothermics.2013.11.004.

Podgorney, R, J Ketilsson, T Driesner, and K Regenauer-Lieb. 2011. "Next Generation of Geothermal Reservoir Simulators: A Draft-Summary of the Recommendations from the International Partnership for Geothermal Technology Reservoir Modeling Working Group," GRC Transactions, 35.

Podgorney, RK, H Huang, and D Gaston. 2010. A Fully-Coupled, Implicit, Finite Element Model for Simultaneously Solving Multiphase Fluid Flow, Heat Transport, and Rock Deformation. In Proceedings of Geothermal Resources Council Annual Meeting, Sacramento, CA,

Pollard, DD and G Holzhausen. 1979. "On the mechanical interaction between a fluid-filled fracture and the earth's surface," Tectonophysics, 53(1-2):27-57. doi:Doi 10.1016/0040-1951(79)90353-6.

Pommier, S, A Gravouil, N Moes, and A Combescure. 2011. Extended Finite Element Method for Crack Propagation. Wiley.

Pruess, K. 1983. Earth Sciences Division Annual Report 1982

, Lawrence Berkeley National Laboratory, Berkeley, California.

Pruess, K. 1987. TOUGH User's Guide, Lawrence Berkeley National Laboratory, Berkeley, CA.

Pruess, K, J Garcia, T Kovscek, C Oldenburg, J Rutqvist, C Steefel, and TF Xu. 2004. "Code intercomparison builds confidence in numerical simulation models for geologic disposal Of $\mathrm{CO} 2$," Energy, 29(9-10):1431-1444. doi:10.1016/j.energy.2004.03.077.

Pruess, K and TN Narasimhan. 1985. "A Practical Method for Modeling Fluid and Heat-Flow in Fractured Porous-Media," Society of Petroleum Engineers Journal, 25(1):14-26.

Pruess, K, C Oldenburg, and GJ Moridis. 2011. TOUGH2 User's Guide, Version 2.1, Lawrence Berkeley National Laboratory, Berkeley, CA. 
Rahman, MK, MM Hossain, and SS Rahman. 2002. "A shear-dilation-based model for evaluation of hydraulically stimulated naturally fractured reservoirs," International Journal for Numerical and Analytical Methods in Geomechanics, 26(5):469-497. doi:DOI 10.1002/nag.208.

Ribeiro, LH, H Li, and JE Bryant. "Use of a CO2-Hybrid Fracturing Design to Enhance Production from Unpropped Fracture Networks," Society of Petroleum Engineers, SPE.

Rice, JR. 1993. "Spatiotemporal Complexity of Slip on a Fault," Journal of Geophysical Research-Solid Earth, 98(B6):9885-9907. doi:Doi 10.1029/93jb00191.

Rinaldi, AP, J Rutqvist, EL Sonnenthal, and TT Cladouhos. 2015. "Coupled THM Modeling of Hydroshearing Stimulation in Tight Fractured Volcanic Rock," Transport in Porous Media, 108(1):131150. doi:10.1007/s11242-014-0296-5.

Roegiers, JJ, CT Montgomery, and TD Wiles. 1982. "Displacement Discontinuity - a New Approach to Modeling Hydraulic Fractures," Journal of Canadian Petroleum Technology, 21(2):44-44.

Rutqvist, J. 2011. "Status of the TOUGH-FLAC simulator and recent applications related to coupled fluid flow and crustal deformations," Computers \& Geosciences, 37(6):739-750. doi:DOI

10.1016/j.cageo.2010.08.006.

Rutqvist, J, J Birkholzer, F Cappa, and CF Tsang. 2007. "Estimating maximum sustainable injection pressure during geological sequestration of $\mathrm{CO} 2$ using coupled fluid flow and geomechanical fault-slip analysis," Energy Conversion and Management, 48(6):1798-1807. doi:DOI

10.1016/j.enconman.2007.01.021.

Rutqvist, J, F Cappa, AP Rinaldi, and M Godano. 2014a. "Modeling of induced seismicity and ground vibrations associated with geologic $\mathrm{CO} 2$ storage, and assessing their effects on surface structures and human perception," International Journal of Greenhouse Gas Control, 24:64-77. doi:DOI 10.1016/j.ijggc.2014.02.017.

Rutqvist, J, PF Dobson, J Garcia, C Hartline, P Jeanne, CM Oldenburg, DW Vasco, and M Walters. 2015a. "The Northwest Geysers EGS Demonstration Project, California: Pre-stimulation Modeling and Interpretation of the Stimulation," Mathematical Geosciences, 47(1):3-29. doi:DOI 10.1007/s11004-0139493-y.

Rutqvist, J, HM Kim, DW Ryu, JH Synn, and WK Song. 2012. "Modeling of coupled thermodynamic and geomechanical performance of underground compressed air energy storage in lined rock caverns," International Journal of Rock Mechanics and Mining Sciences, 52:71-81. doi:DOI 10.1016/j.ijrmms.2012.02.010.

Rutqvist, J and GJ Moridis. 2009. "Numerical Studies on the Geomechanical Stability of Hydrate-Bearing Sediments," Spe Journal, 14(2):267-282.

Rutqvist, J, AP Rinaldi, F Cappa, and GJ Moridis. 2015b. "Modeling of fault activation and seismicity by injection directly into a fault zone associated with hydraulic fracturing of shale-gas reservoirs," Journal of Petroleum Science and Engineering, 127:377-386. doi:DOI 10.1016/j.petrol.2015.01.019. 
Rutqvist, J and C-F Tsang. 2003. Tough-Flac: A Numerical Simulator For Analysis Of Coupled ThermalHydrologic-Mechanical Processes In Fractured And Porous Geological Media Under Multi-Phase Flow Conditions. In Proceedings of TOUGH symposium 2003, Lawrence Berkeley National Laboratory, Berkeley, CA,

Rutqvist, J, YS Wu, CF Tsang, and G Bodvarsson. 2002. "A modeling approach for analysis of coupled multiphase fluid flow, heat transfer, and deformation in fractured porous rock," International Journal of Rock Mechanics and Mining Sciences, 39(4):429-442. doi:Pii S1365-1609(02)00022-9 Doi 10.1016/S1365-1609(02)00022-9.

Rutqvist, J, LG Zheng, F Chen, HH Liu, and J Birkholzer. 2014b. "Modeling of Coupled Thermo-HydroMechanical Processes with Links to Geochemistry Associated with Bentonite-Backfilled Repository Tunnels in Clay Formations," Rock Mechanics and Rock Engineering, 47(1):167-186. doi:DOI 10.1007/s00603-013-0375-x.

Salamon, MDG. 1963. "Elastic analysis of displacements and stresses induced by mining of reef deposits, Part II," J. South Afr. Inst. Min. Metall., 64(6):197-218.

Scheibe, TD, MD White, and SK White. 2013. Outcomes of the 2013 GTO Workshop on Geothermal Code Comparison, Pacific Northwest National Laboratory.

Settgast, R, S Johnson, W S., P Fu, J White, and FJ Ryerson. 2014. GEOS Quarterly Update and Roadmap, Lawrence Livermore National Laboratory.

Shou, KJ and SL Crouch. 1995. "A Higher-Order Displacement Discontinuity Method for Analysis of Crack Problems," International Journal of Rock Mechanics and Mining Sciences \& Geomechanics Abstracts, 32(1):49-55. doi:Doi 10.1016/0148-9062(94)00016-V.

Smith, JT, EL Sonnenthal, and T Cladouhos. 2015. Thermal-hydrological-mechanical modelling of shear stimulation at Newberry Volcano, Oregon. In Proceedings of 49th US Rock Mechanics / Geomechanics Symposium, San Francisco, California, American Rock Mechanics Association

Sonnenthal, E, A Ito, N Spycher, M Yui, J Apps, Y Sugita, M Conrad, and S Kawakami. 2005.

"Approaches to modeling coupled thermal, hydrological, and chemical processes in the Drift Scale Heater Test at Yucca Mountain," International Journal of Rock Mechanics and Mining Sciences, 42(5-6):698719. doi:DOI 10.1016/j.ijrmms.2005.03.009.

Sonnenthal, E, N Spycher, O Callahan, T Cladouhos, and S Petty. 2012. A thermal-hydrological-chemical model for the Enhanced Geothermal System Demonstration Project at Newberry Volcano, Oregon. In Proceedings of Thirty-Seventh Workshop on Geothermal Reservoir Engineering, Stanford University, Stanford, California,

Sonnenthal, EL, JT J.T. Smith, T Cladouhos, J Kim, and L Yang. 2015. Thermal-HydrologicalMechanical-Chemical Modeling of the 2014 EGS Stimulation Experiment at Newberry Volcano, Oregon. In Proceedings of Fortieth Workshop on Geothermal Reservoir Engineering, Stanford University, Stanford, California,

Sonnenthal, EL, N Spycher, T Xu, L Zheng, N Miller, and K Pruess. 2014. TOUGHREACT V3.0-OMP. Accessed on May 6, 2015, at http://esd.lbl.gov/research/projects/tough/software/toughreact.html (last updated 
Spycher, NF, EL Sonnenthal, and JA Apps. 2003. "Fluid flow and reactive transport around potential nuclear waste emplacement tunnels at Yucca Mountain, Nevada," Journal of Contaminant Hydrology, 623:653-673. doi:Doi 10.1016/S0169-7722(02)00183-3.

Stanford Special Panel. 1980. Special Panel on Geothermal Model Intercomparison Study. In Proceedings of Sixth Workshop on Geothermal Reservoir Engineering, Stanford, California, Stanford University,

Steefel, CI, CaJ Appelo, B Arora, D Jacques, T Kalbacher, O Kolditz, V Lagneau, PC Lichtner, KU Mayer, JCL Meeussen, S Molins, D Moulton, H Shao, J Simunek, N Spycher, SB Yabusaki, and GT Yeh. 2015. "Reactive transport codes for subsurface environmental simulation," Computational Geosciences, 19(3):445-478. doi:10.1007/s10596-014-9443-x.

Steefel, CI, CaJ Appelo, B Arora, D Jacques, T Kalbacher, O Kolditz, V Lagneau, PC Lichtner, KU Mayer, JCL Meeussen, S Molins, D Moulton, H Shao, J Šimůnek, N Spycher, SB Yabusaki, and GT Yeh. 2014. "Reactive transport codes for subsurface environmental simulation," Computational Geosciences:134. doi:10.1007/s10596-014-9443-X.

Steefel, CI and AC Lasaga. 1994. "A Coupled Model for Transport of Multiple Chemical-Species and Kinetic Precipitation Dissolution Reactions with Application to Reactive Flow in Single-Phase Hydrothermal Systems," American Journal of Science, 294(5):529-592.

STOMP User Guide. 2015. Accessed on March 19, 2015, at stomp.pnnl.gov/user_guide/STOMP guide.stm (last updated

Tang, CA, LG Tham, PKK Lee, TH Yang, and LC Li. 2002. "Coupled analysis of flow, stress and damage (FSD) in rock failure," International Journal of Rock Mechanics and Mining Sciences, 39(4):477489. doi:Pii S1356-1609(02)00023-0.

Taron, J and D Elsworth. 2009. "Thermal-hydrologic-mechanical-chemical processes in the evolution of engineered geothermal reservoirs," International Journal of Rock Mechanics and Mining Sciences, 46(5):855-864. doi:DOI 10.1016/j.ijrmms.2009.01.007.

Taron, J and D Elsworth. 2010a. "Constraints on compaction rate and equilibrium in the pressure solution creep of quartz aggregates and fractures: Controls of aqueous concentration," Journal of Geophysical Research-Solid Earth, 115. doi:Artn B07211

Doi 10.1029/2009jb007118.

Taron, J and D Elsworth. 2010b. "Coupled mechanical and chemical processes in engineered geothermal reservoirs with dynamic permeability," International Journal of Rock Mechanics and Mining Sciences, 47(8):1339-1348. doi:DOI 10.1016/j.ijrmms.2010.08.021.

Tchelepi, H and Y Zhou. Multi-GPU Parallelization of Nested Factorization for Solving Large Linear Systems. In Proceedings Society of Petroleum Engineers, SPE.

Tsang, CF, O Stephansson, LR Jing, and F Kautsky. 2009. "DECOVALEX Project: from 1992 to 2007," Environmental Geology, 57(6):1221-1237. doi:10.1007/s00254-008-1625-1.

U.S. Doe. 2010. Geothermal Technology Program, 2010 Peer Review Report. 
U.S. Doe. 2011. Geothermal Technology Program, 2011 Peer Review Report.

U.S. Doe. 2015. Geothermal Technology Program, 2015 Peer Review Report.

Vandamme, L and JH Curran. 1989. "A 3-Dimensional Hydraulic Fracturing Simulator," International Journal for Numerical Methods in Engineering, 28(4):909-927. doi:DOI 10.1002/nme.1620280413.

Vandamme, LM and JC Roegiers. 1990. "Poroelasticity in Hydraulic Fracturing Simulators," Journal of Petroleum Technology, 42(9):1199-1203.

Verma, A and K Pruess. 1988. "Thermohydrological Conditions and Silica Redistribution near HighLevel Nuclear Wastes Emplaced in Saturated Geological Formations," Journal of Geophysical ResearchSolid Earth and Planets, 93(B2):1159-1173. doi:DOI 10.1029/JB093iB02p01159.

Vinsome, PKW and J Westerveld. 1980. "A Simple Method for Predicting Cap and Base Rock HeatLosses in Thermal Reservoir Simulators," Journal of Canadian Petroleum Technology, 19(3):87-90.

Voskov, DV and HA Tchelepi. 2012. "Comparison of nonlinear formulations for two-phase multicomponent EoS based simulation," Journal of Petroleum Science and Engineering, 82-83:101-111. doi:DOI 10.1016/j.petrol.2011.10.012.

Voskov, DV, HA Tchelepi, and R Younis. General Nonlinear Solution Strategies for Multiphase Multicomponent EoS Based Simulation. In Proceedings Society of Petroleum Engineers, SPE.

Voskov, DV and O Volkov. Advanced Strategies of Forward Simulation for Adjoint-Based Optimization. In Proceedings Society of Petroleum Engineers, SPE.

Wagner, W, JR Cooper, A Dittmann, J Kijima, HJ Kretzschmar, A Kruse, R Mareš, K Oguchi, H Sato, I StöCker, O ŠIfner, Y Takaishi, I Tanishita, J TrüBenbach, and T Willkommen. 2000. "The IAPWS Industrial Formulation 1997 for the Thermodynamic Properties of Water and Steam," Journal of Engineering for Gas Turbines and Power, 122(1):150-184. doi:10.1115/1.483186.

Wanner, C, JL Druhan, RT Amos, P Alt-Epping, and CI Steefel. 2015. "Benchmarking the simulation of Cr isotope fractionation," Computational Geosciences, 19(3):497-521. doi:10.1007/s10596-014-9436-9.

White, JA, L Chiaramonte, S Ezzedine, W Foxall, Y Hao, A Ramirez, and W Mcnab. 2014a. "Geomechanical behavior of the reservoir and caprock system at the In Salah CO2 storage project," Proceedings of the National Academy of Sciences of the United States of America, 111(24):8747-8752. doi:10.1073/pnas.1316465111.

White, M and WS Lee. 2014. Guest Molecule Exchange Kinetics for the 2012 Ignik Sikumi Gas Hydrate Field Trial. In Proceedings Offshore Technology Conference, OTC.

White, M and M Oostrom. 2000. STOMP Subsurface Transport Over Multiple Phases Version 2.0 Theory Guide, PNNL-12030.

White, MD, DH Bacon, BP Mcgrail, DJ Watson, SK White, and ZF Zhang. 2012. STOMP Subsurface Transport Over Multiple Phases: STOMP-CO2 and STOMP-CO2e Guide: Version 1.0, PNNL-21268. 
White, MD, D Bahrami, G Danko, D Elsworth, P Fu, JK Furtney, A Ghassemi, RN Horne, SM Kelkar, MW Mcclure, RK Podgorney, J Rutqvist, and E Sonnethal. 2015a. "Numerical simulators for enhanced geothermal systems: GTO-CCS codes," Conputational Geosciences.

White, MD and BP Mcgrail. 2005. STOMP Subsurface Transport Over Multiple Phases Version 1.0 Addendum: ECKEChem Equilibrium-Conservation-Kinetic Equation Chemistry and Reactive Transport, PNNL-15482; Other: 3573; CE0300000; TRN: US201009\%\%272 United States 10.2172/976999Other: 3573; CE0300000; TRN: US201009\%\%272Thu Jun 03 07:19:20 EDT 2010PNNLEnglish.

White, MD, BJ Mcpherson, RB Grigg, W Ampomah, and MS Appold. 2014b. "Numerical Simulation of Carbon Dioxide Injection in the Western Section of the Farnsworth Unit," Energy Procedia, 63(0):78917912. doi:http://dx.doi.org/10.1016/j.egypro.2014.11.825.

White, MD and M Oostrom. 2006. STOMP Subsurface Transport Over Multiple Phases, Version 4.0, User's Guide, PNNL-15782.

White, MD, M Oostrom, and RJ Lenhard. 1995. "Modeling Fluid-Flow and Transport in Variably Saturated Porous-Media with the Stomp Simulator .1. Nonvolatile 3-Phase Model Description," Advances in Water Resources, 18(6):353-364. doi:Doi 10.1016/0309-1708(95)00018-E.

White, MD and BR Phillips. 2015. Code Comparison Study Fosters Confidence in the Numerical Simulation of Enhanced Geothermal Systems. In Proceedings of Fourtieth Workshop on Geothermal Reservoir Engineering Stanford University, Stanford, CA,

White, MD and AL Ward. 2005. Numerical simulation of surface barriers for shrub-steppe ecoregions. In Proceedings of American Geophysical Union Hydrology Days, Fort Collins, Colorado,

White, SK, S Purohit, and L Boyd. 2015b. Using GTO-Velo to Facilitate Communication and Sharing of Simulation Results in Support of

the Geothermal Technologies Office Code Comparison Study. In Proceedings of Fourtieth Workshop on Geothermal Reservoir Engineering, Stanford University, Stanford, California,

Widlowski, JL, B Pinty, M Lopatka, C Atzberger, D Buzica, M Chelle, M Disney, JP GastelluEtchegorry, M Gerboles, N Gobron, E Grau, H Huang, A Kallel, H Kobayashi, PE Lewis, W Qin, M Schlerf, J Stuckens, and D Xie. 2013. "The fourth radiation transfer model intercomparison (RAMI-IV): Proficiency testing of canopy reflectance models with ISO-13528," Journal of Geophysical Research: Atmospheres, 118(13):6869-6890. doi:10.1002/jgrd.50497.

Wilder, JW, GJ Moridis, SJ Wilson, M Kurihara, MD White, Y Masuda, BJ Anderson, TS Collett, RB Hunter, H Narita, M Pooladi-Darvish, K Rose, and R Boswell. 2008. An International Effort to Compare Gas Hydrate Reservoir Simulators. In Proceedings of 6th International Conference on Gas Hydrates (ICGH 2008), Vancouver, British Columbia, Canada, ICGH,

Willis-Richards, J, K Watanabe, and H Takahashi. 1996. "Progress toward a stochastic rock mechanics model of engineered geothermal systems," Journal of Geophysical Research-Solid Earth, 101(B8):1748117496. doi:Doi 10.1029/96jb00882.

Wood, WW. 2009. "Enhanced Geothermal Systems: An Opportunity for Hydrogeology," Ground Water, 47(6):751-751. doi:DOI 10.1111/j.1745-6584.2009.00573.x. 
$\mathrm{Xu}$, TF, JA Apps, and K Pruess. 2004a. "Numerical simulation of CO2 disposal by mineral trapping in deep aquifers," Applied Geochemistry, 19(6):917-936. doi:DOI 10.1016/j.apgeochem.2003.11.003.

$\mathrm{Xu}, \mathrm{TF}, \mathrm{Y}$ Ontoy, P Molling, N Spycher, M Parini, and K Pruess. 2004b. "Reactive transport modeling of injection well scaling and acidizing at Tiwi field, Philippines," Geothermics, 33(4):477-491. doi:DOI 10.1016/j.geothermics.2003.09.012.

$\mathrm{Xu}, \mathrm{TF}$ and K Pruess. 2001. "Modeling multiphase non-isothermal fluid flow and reactive geochemical transport in variably saturated fractured rocks: 1. Methodology," American Journal of Science, 301(1):1633. doi:DOI 10.2475/ajs.301.1.16.

$\mathrm{Xu}, \mathrm{TF}$ and K Pruess. 2004. "Numerical Simulation of Injectivity Effects of Mineral Scaling and Clay Swelling in a Fractured Geothermal Reservoir." Lawrence Berkeley National Laboratory, Berkeley, CA.

$\mathrm{Xu}, \mathrm{TF}$, E Sonnenthal, N Spycher, and K Pruess. 2006. "TOUGHREACT - A simulation program for non-isothermal multiphase reactive geochemical transport in variably saturated geologic media:

Applications to geothermal injectivity and CO2 geological sequestration," Computers \& Geosciences, 32(2):145-165. doi:DOI 10.1016/j.cageo.2005.06.014.

Xu, TF, E Sonnenthal, N Spycher, K Pruess, G Brimhall, and J Apps. 2001. "Modeling multiphase nonisothermal fluid flow and reactive geochemical transport in variably saturated fractured rocks: 2 . Applications to supergene copper enrichment and hydrothermal flows," American Journal of Science, 301(1):34-59. doi:DOI 10.2475/ajs.301.1.34.

Xu, TF, N Spycher, E Sonnenthal, GX Zhang, LE Zheng, and K Pruess. 2011. "TOUGHREACT Version 2.0: A simulator for subsurface reactive transport under non-isothermal multiphase flow conditions," Computers \& Geosciences, 37(6):763-774. doi:DOI 10.1016/j.cageo.2010.10.007.

Younis, RM. 2011. "Modern advances in software and solution algorithms for reservoir simulation," Ph.D., Stanford University.

Zaydullin, R, D Voskov, and HA Tchelepi. "Nonlinear Formulation Based on an Equation-of-State Free Method for Compositional Flow Simulation." doi:10.2118/146989-PA.

Zhang, ZF, LR Zhong, MD White, and JE Szecsody. 2012. "Experimental Investigation of the Effective Foam Viscosity in Unsaturated Porous Media," Vadose Zone Journal, 11(4). doi:DOI 10.2136/vzj2011.0190.

Zhou, Y. 2012. "Parallel general-purpose reservoir simulation with coupled reservoir models and multisegment wells," Ph.D., Stanford University.

Zhou, Y, HA Tchelepi, and BT Mallison. Automatic Differentiation Framework for Compositional Simulation on Unstructured Grids with Multi-Point Discretization Schemes. In Proceedings Society of Petroleum Engineers, SPE.

Zyvoloski, G. 2007. FEHM: A control volume finite element code for simulating subsurface multi-phase multi-fluid heat and mass transfer, Los Alamos National Laboratory. 


\section{Distribution}

No. of

Copies

\section{Electronic Distribution}

Sertac Akar (Sertac.Akar@nrel.gov)

Mahmood Arshad (harshad@mines.edu)

Chad Augustine (Chad.Augustine@nrel.gov)

DavoodBahrami (dbahrami@unr.edu)

Charlotte Barbier (barbiercn@ornl.gov)

Colleen Barton (colleen.barton@bakerhughes.com)

Mary Baumgartner (mary.baumgartner@meliortechnology.com)

Koenraad Beckers (Koenraad.Beckers@nrel.gov)

Jens Birkholzer (jtbirkholzer@lbl.gov)

Lauren Boyd (Lauren.Boyd@ee.doe.gov)

Jan Brezina (jbrezmorf@gmail.com)

Christopher Brown (Christopher.Brown@pnnl.gov)

Donald Brown (dwb@lanl.gov)

Charles Carrigan (carrigan1@1lnl.gov)

Qinglu Cheng (Qinglu.cheng@ou.edu)

Kit-Kwan Chiu (lydiackk@gmail.com)

Carla Co (carlakdc@stanford.edu)

Cari Debra Covell (cari14@ru.is)

Branko Damjanac (branko@itascacg.com)

George Danko (danko@unr.edu)

Christine Detournay (christine@itascag.com)

Derek Elsworth (elsworth@psu.edu)

Yi Fang (yi.fang@psu.edu)

Zachary Frone (Zachary.Frone@EE.doe.gov)

Pengcheng Fu (fu4@1lnl.gov)

Jason Furtney (jfurtney@itascacg.com)

Quan Gan (gan.quan@abdn.ac.uk)

Qian Gao (Qian.gao@ou.edu)

Ahmad Ghassemi (ahmad.ghassemi@ou.edu)

Morgan Greene (rmorgang@ru.is)

Bin Guo (fullofenergy3@gmail.com)

Yue Hao (hao1@1lnl.gov)

Hiro Honda (honda@doc.kyushu-u.ac.jp)

Roland Horne (horne@stanford.edu)

Hai Huang (hai.huang@inl.gov)

Kai Huang (kai.huang@ou.edu)

Kyungjae Im (kxi123@psu.edu)

Kamran Jahanbakhsh (kjahanba@mines.edu)

Jaewon Jang (jjang19@asu.edu)

Patrick Jenny (jenny@ifd.mavt.ethz.ch)

Henry Johnston (Henry.Johnston@nrel.gov)

Sharad Kelkar (sharadmkelkar@gmail.com)

Zoheir Khademian (zkhademi@mines.edu)

Halla Hrund Logadóttir (hallal@ru.is) 
Kevin McCabe (Kevin.McCabe@nrel.gov)

Mark McClure (mark.w.mccl@gmail.com)

Elisabet Metcalfe (Elisabet.Metcalfe@EE.Doe.Gov)

Maruti Kumar Mudunuru (maruti@lanl.gov)

Masami Nakagawa (mnakagaw@mines.edu)

Jack Norbeck (jnorbeck@stanford.edu)

Mitchell Plummer (mitchell.plummer@inl.gov)

Robert Podgorney (robert.podgorney@inl.gov)

Justin Pogacnik (j.pogacnik@auckland.ac.nz)

Yarom Polsky (polskyy@ornl.gov)

Sean Porse (sean.porse@ee.doe.gov)

Sumit Purohit (Sumit.Purohit@pnnl.gov)

Azadeh Riahi (ariahi@itascacg.com)

Ivan Rubio (ivangvr1975@gmail.com)

Jonny Rutqvist (JRutqvist@lbl.gov)

Reza Safari (mrsafari@gmail.com)

Pratap Sahay (pratap@cicese.mx)

Varahanaresh Sesetty (Varahanaresh.Sesetty@ou.edu)

Eric Sonnenthal (ELSonnenthal@lbl.gov)

Jan Stebel (jan.stebel@tul.cz)

Qingfeng Tao (taoqingfeng@gmail.com)

Jeff Tester (jwt54@cornell.edu)

William Vandermeer (William.Vandermeer@ee.doe.gov)

Denis Voskov (d.v.voskov@tudelft.nl)

Shihao Wang (shiwang@mines.edu)

Mark White (Mark.White@pnnl.gov)

Signe White (Signe.White@pnnl.gov)

Jeffrey Winick (JWinick@slb.com)

Philip Winterfeld (pwinterf@mines.edu)

Jessica Wisse (jessica.wisse@pnnl.gov)

Yang Wong (zhiyangw@stanford.edu)

Yidong Xia (yidong.xia@inl.gov)

Tianfu Xu (Tianfu_Xu@jlu.edu.cn)

Bill Yu (xxy21@case.edu)

Yilong Yuan (yuanyl14@mails.jlu.edu.cn)

Quanlin Zhou (qzhou@lbl.gov)

*All distributions will be electronic in PDF format. 



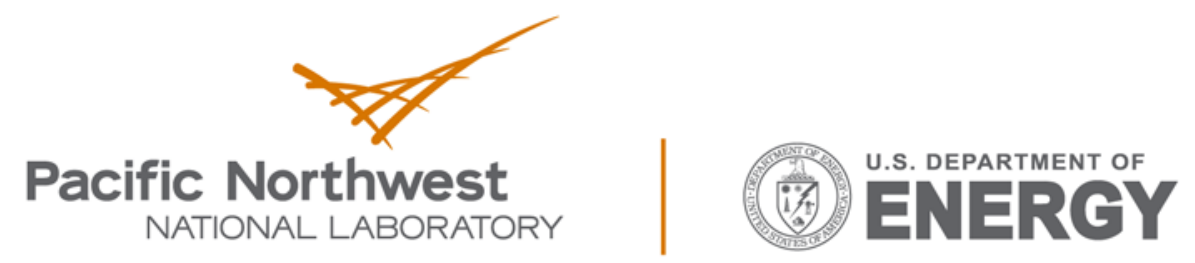

Proudly Operated by Battelle Since 1965

902 Battelle Boulevard

P.O. Box 999

Richland, WA 99352

1-888-375-PNNL (7665)

www.pnnl.gov 